\title{
A Measurement of the Tau Michel Parameters at SLD
}

\author{
J. Quigley
}

SLAC-Report-506

May 1997

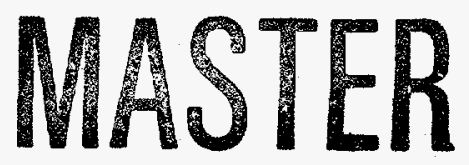

Prepared for the Department of Energy

under contract number DE-AC03-76SF00515

DISTRIBUTION OF THIS DOCUMENT IS UNLIMITED 


\section{DISCLAMMER}

Portions of this document may be illegible in electronic image products. Images are produced from the best available original document. 
This document and the material and data contained therein, was developed under sponsorship of the United States Government. Neither the United States nor the Department of Energy, nor the Leland Stanford Junior University, nor their employees, nor their respective contractors, subcontractors, or their employees, makes any warranty, express or implied, or assumes any liability or responsibility for accuracy, completeness or usefulness of any information, apparatus, product or process disclosed, or represents that its use will not infringe privately-owned rights. Mention of any product, its manufacturer, or suppliers shall not, not is it intended to, imply approval, disapproval, or fitness for any particular use. A royalty-free, nonexclusive right to use and disseminate same for any purpose whatsoever, is expressly reserved to the United States and the University.

This report was prepared as an account of work sponsored by an agency of the United States Government. Neither the United States Government nor any agency thereof, nor any of their employees, makes any warranty, express or implied, or assumes any legal liability or responsibility for the accuracy, completeness, or usefulness of any information, apparatus, product, or process disclosed, or represents that its use would not infringe privately owned rights. Reference herein to any specific commercial product, process, or service by trade name, trademark, manufacturer, or otherwise does not necessarily constitute or imply its endorsement, recommendation, or favoring by the United States Government or any agency thereof. The views and opinions of authors expressed herein do not necessarily state or reflect those of the United States Government or any agency thereof. 
A Measurement of the Tau Michel Parameters at SLD*

\author{
J. Quigley \\ Stanford Linear Accelerator Center \\ Stanford University \\ Stanford, CA 94309 \\ RECEIVED \\ AUG 111997 \\ OSTI \\ MASTER
}

\title{
SLAC-Report-506
}

May 1997

Prepared for the Department of Energy under contract number DE-AC03-76SF00515

Printed in the United States of America. Available from the National Technical Information Service, U.S. Department of Commerce, 5285 Port Royal Road, Springfield, VA 22161.

Ph.D. thesis, Massachusetts Institute of Technology. 


\title{
A Measurement of the Tau Michel Parameters at SLD.
}

\author{
by \\ James A. Quigley \\ Submitted to the Department of Physics on May 2, 1997, \\ in partial fulfillment of the requirements for the degree of \\ Doctor of Philosophy.
}

\begin{abstract}
This thesis presents a measurement of the tau Michel parameters. This measurement utilizes the highly polarized SLC electron beam to extract these quantities directly from the measured tau decay spectra using the 1993-95 SLD sample of 4528 tau pair events. The results are $\rho^{e}=0.71 \pm 0.14 \pm 0.05, \xi^{e}=1.16 \pm 0.52 \pm 0.06$, and $(\xi \delta)^{e}=0.85 \pm 0.43 \pm 0.08$ for tau decays to electrons and $\rho^{\mu}=0.54 \pm 0.28 \pm 0.14, \eta^{\mu}=-0.59 \pm 0.82 \pm 0.45, \xi^{\mu}=0.75 \pm 0.50 \pm 0.14$, and $(\xi \delta)^{\mu}=0.82 \pm 0.32 \pm 0.07$ for tau decays to muons. Combining all leptonic tau decays gives $\rho=0.72 \pm 0.09 \pm 0.03, \xi=1.05 \pm 0.35 \pm 0.04$, and $\xi \delta=0.88 \pm 0.27 \pm 0.04$. These results agree well with the current world average and the Standard Model.
\end{abstract}

Thesis Supervisor: Richard K. Yamamoto

Title: Professor of Physics 


\section{Acknowledgments}

At first this was going to say "I acknowledge no one but myself," but that would, of course, be untrue. Next, this was going to be a voluminous epistle to the glories of everyone I've associated with during my seven years of graduate school. In the end, since I just want to get this thing finished and submitted, this is just going to be a few short words. Since I'm writing this from the rather skewed perspective at the end of seven years of work, I'll probably leave out quite a few important people.

First of all, I have to acknowledge my advisor, Dick Yamamoto, for providing much useful advice and shepherding us all through our qualifying exams and beyond. Secondly, I thank all of my fellow LQS group members, Eric, Mike, Oliver and Ray, for providing companionship, advice, and keeping me sane through all these years. I also have to thank the SLD tau group for providing a excellent working environment. In particular I have to thank John Venuti for getting me started, Mourad for keeping me going, Nik and Erez for being excellent co-workers, and Jim Johnson for providing stability. Also, I have to thank the SLC staff for providing us with Physics events, and the SLD collaboration for making it possible to analyze them. Additionally, I should thank Phil and Amit for making SLAC a little more entertaining.

In a less Physics oriented vein, I have to thank the SLAC softball team for providing a much needed diversion and an excuse to go the Goose every so often. Special thanks go to Mike Woods for organizing it all. Also, I have to acknowledge the Gang, Cherie, Dave, Greg, Suzanne, etc. for providing much fun with Bridge and Tequila. In addition, I have to 
acknowledge the SLAC Canadian contingent, which has included several good friends, including Cherie, Suzanne, and Nicola.

Getting away from SLAC, I have to thank the boys from Rains 2e, Clark, Dave and Hu for a lot of Frisbee golf among other things. I also have to thank the Tuesday Ultimate group for providing some much needed exercise. Thanks to my fellow Woodsiders Chip and Andrew for being great housemates and joining me in putting up with all that Drees nonsense. Special thanks go to the Posse for providing an annual vacation worth talking about. In particular I have to thank Dave Donohue for making the Posse possible and for the nicknames. Also, I have to thank John "Saucy Jack" Garrett, Jim Williams and Mike “Smokin' Gun" Jones for being good friends. Special thanks, I think, go to Mike for introducing me to Tim and the Wrecking Crew.

Finally, I have to acknowledge my family. Thanks to my parents for bringing me up right and giving me the ability to accomplish many things including this work. A special thanks goes to my sister Susan for always being encouraging. 


\section{Table of Contents}

Abstract
Acknowledgments
List of Figures
List of Tables
Introduction
1 Physics Background
1.1 .1 Tree Level Fermion Pair Production Cross Sections

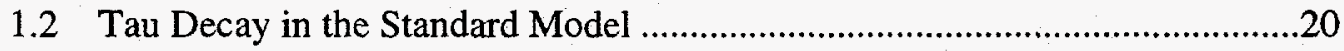

1.3 More General Parameterizations for Leptonic Tau Decays............................22

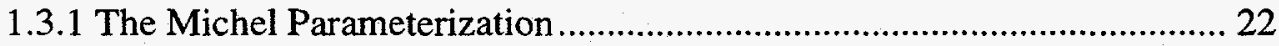

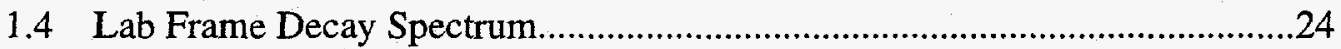

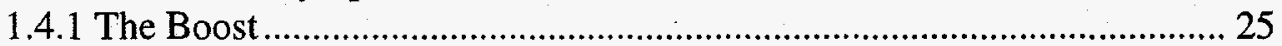

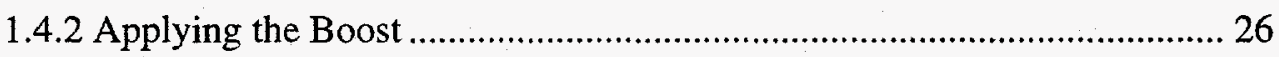

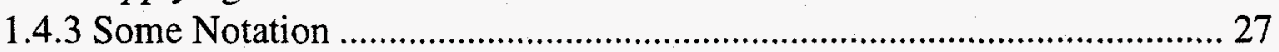

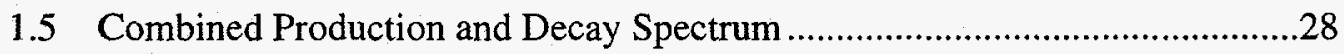

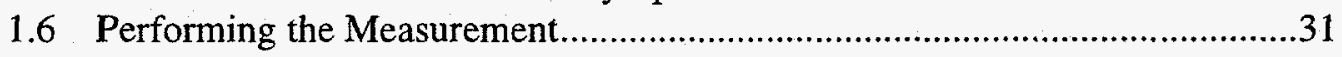

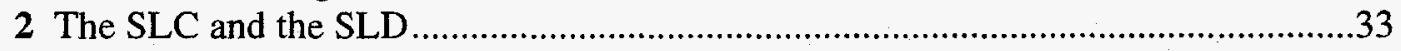

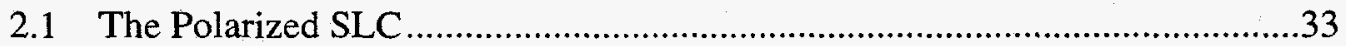

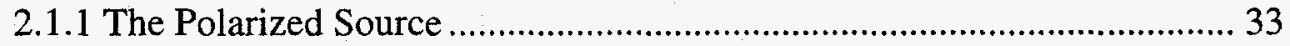

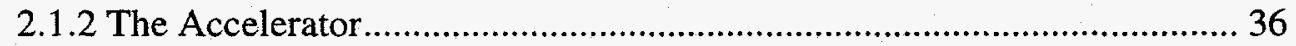

2.1.3 The Energy Spectrometers ................................................................ 39

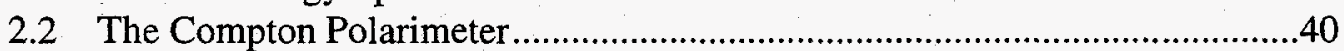

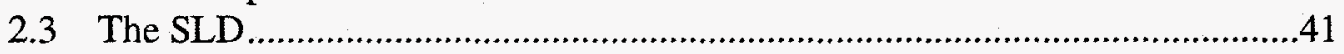

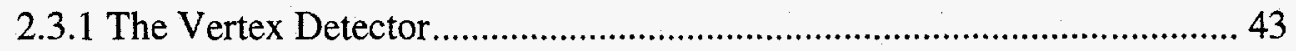

2.3.2 The Central Drift Chamber ................................................................. 45

2.3.3 The Barrel Cherenkov Ring Imaging Detector ...................................... 46

2.3.4 The Liquid Argon Calorimeter. ........................................................ 48

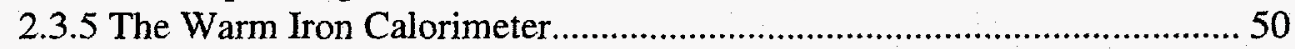

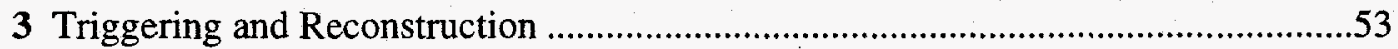

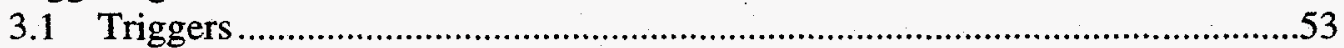

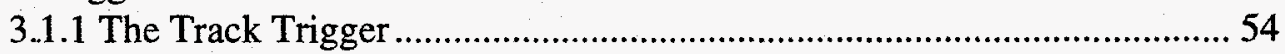

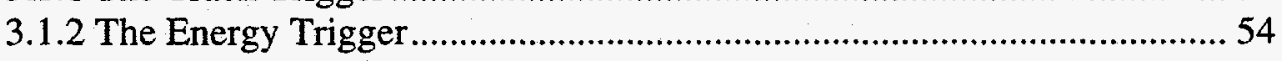

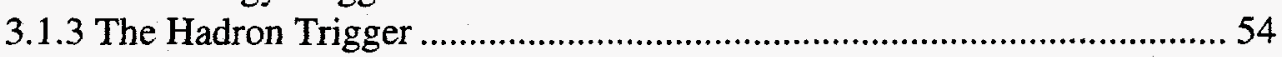

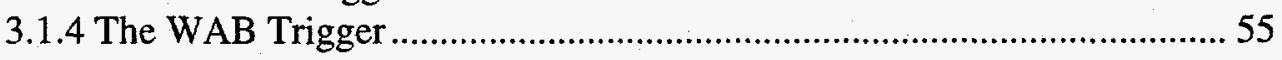

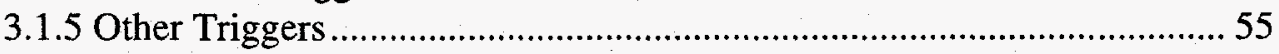

3.2 Trigger Efficiencies for Tau Pair Events …..............................................55 
3.2.1 Monte Carlo Simulation of trigger efficiency ......................................... 55

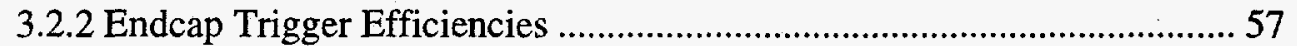

3.2.3 Trigger Efficiencies from Correlations in the Data ............................... 58

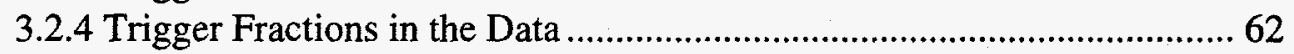

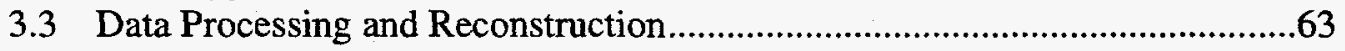

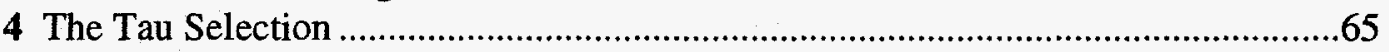

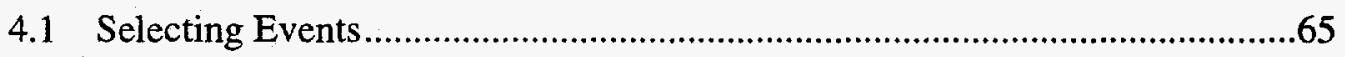

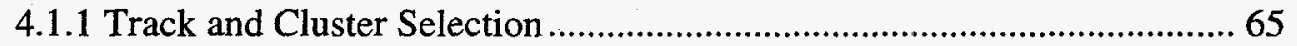

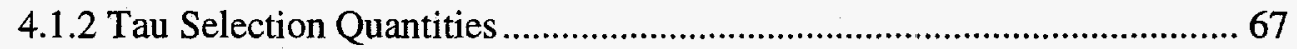

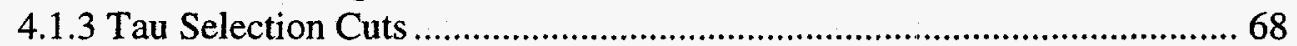

4.1.4 Plots of Tau Filter Quantities for Monte Carlo and Data........................ 70-

4.2 Efficiency and Backgrounds for the Tau Filter.............................................75

4.2.1 Tau Selection Efficiencies from Monte Carlo ......................................... 75

4.2.2 Backgrounds from the Monte Carlo .................................................... 78

4.2.3 Comparison of Efficiency and Background to the Data ......................... 78

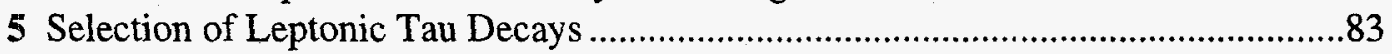

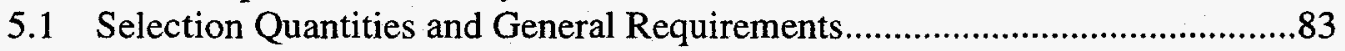

5.1.1 Muon Identification Requirements ................................................... 84

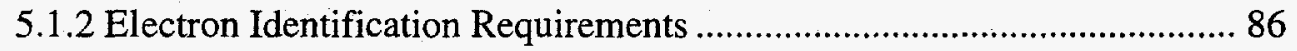

5.1.3 Plots of Particle Identification Quantities ............................................. 87

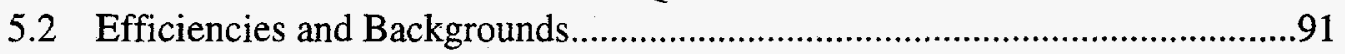

5.2.1 Electron Selection Efficiency and Background ................................... 91

5.2.2 Muon Selection Efficiency and Background ....................................... 93

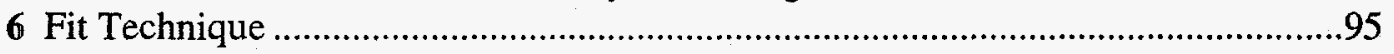

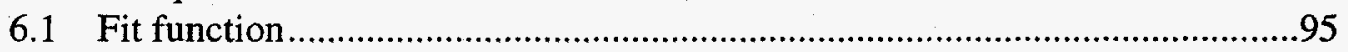

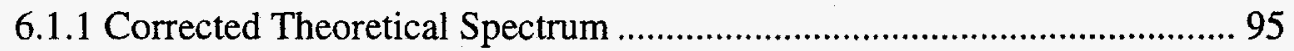

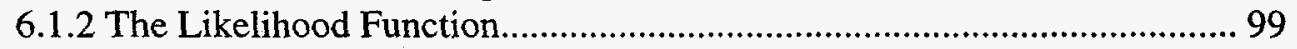

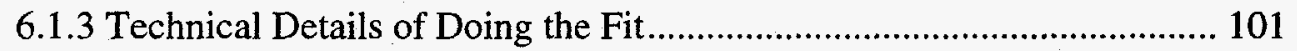

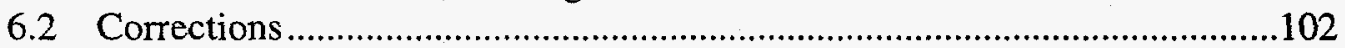

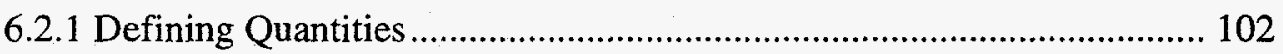

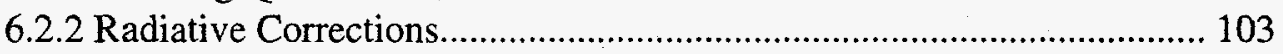

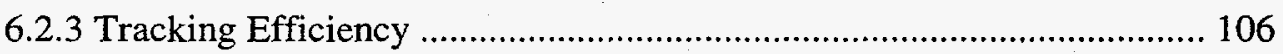

6.2.4 Electron and Muon Angular Resolution ......................................... 107

6.2.5 Muon Momentum Resolution ......................................................... 112

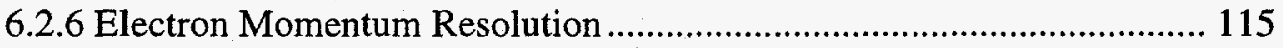

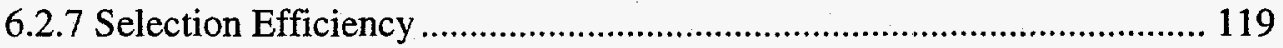

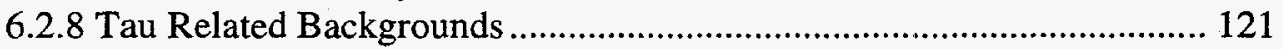

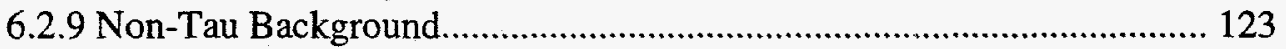

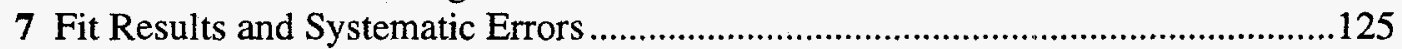

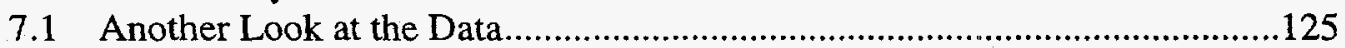

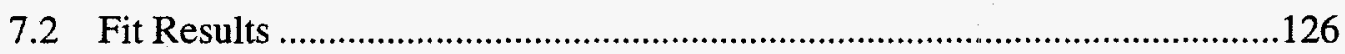

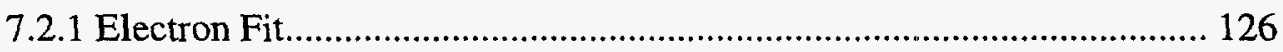

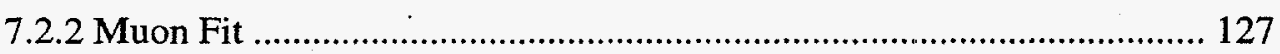

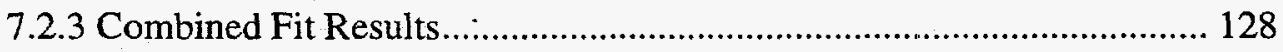

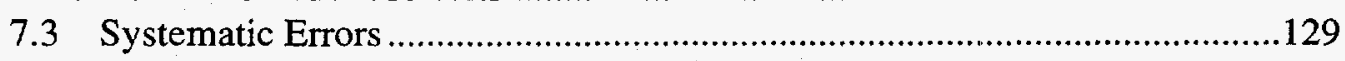

7.3.1 Errors due to Correction Functions and Input Parameters.................... 130 


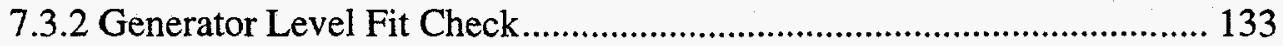

7.3.3 Full Monte Carlo Fit Check .................................................................... 134

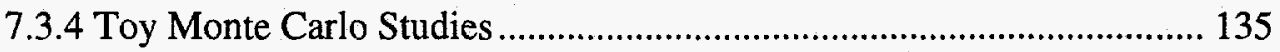

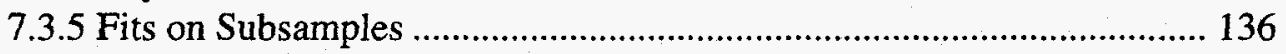

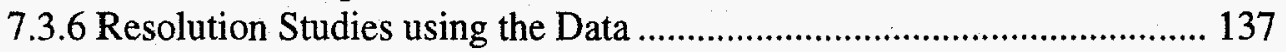

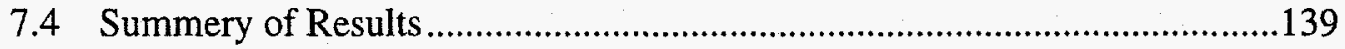

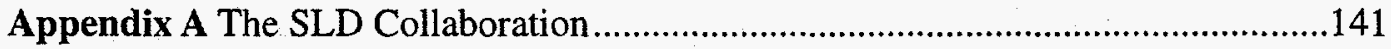

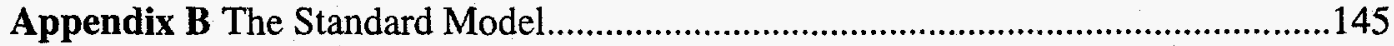

B.1 An Overview of the Standard Model ........................................................145

B.1.1 Known Particles and Fields ................................................................145

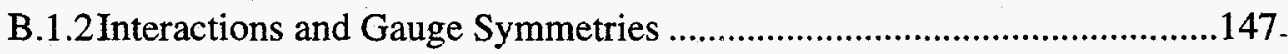

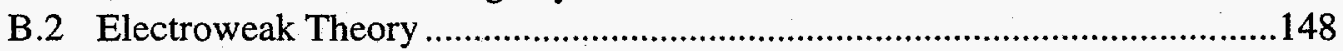

B.2.3 Massless Lagrangian ...................................................................149

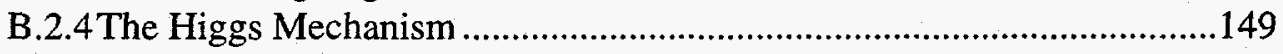

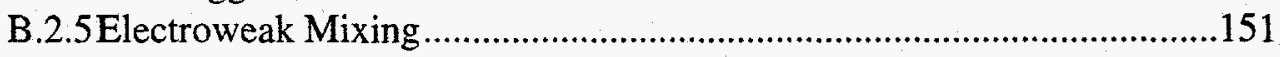

2.2.6 Feynman Rules for the Electroweak Interactions ................................ 152

Appendix C Tau Decay Parameterization for a Generalized Weak Interaction ..........155

Appendix D Tau Neutrino Helicity from Leptonic Tau Decays................................159

Appendix E LAC Electro-Magnetic Energy Calibration .........................................161

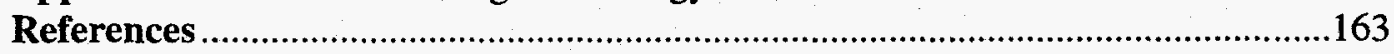




\section{List of Figures}

Figure 1.1: Tree level fermion production diagrams. ................................................18

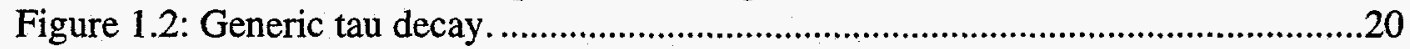

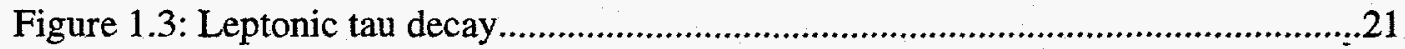

Figure 1.4: Tau Polarization vs. $\cos \theta$ for various beam polarizations..........................29

Figure 1.5: Polarization independent spectral functions.............................................30

Figure 1.6: Polarization dependant spectral functions. ..............................................30

Figure 1.7: Left and right-handed lab frame tau Michel spectra. ..................................31

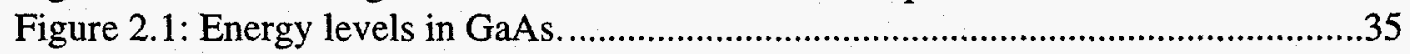

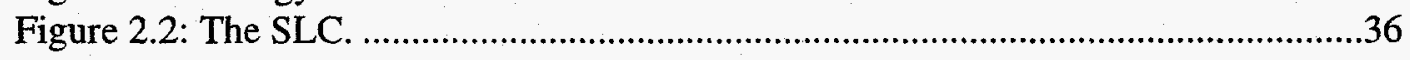

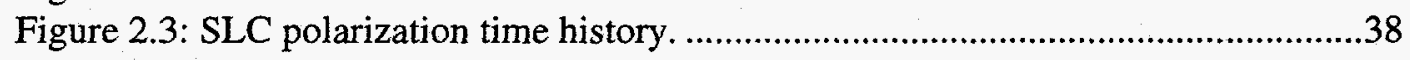

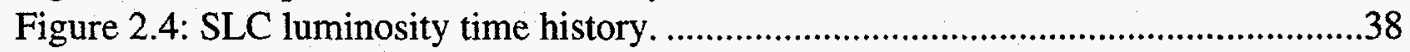

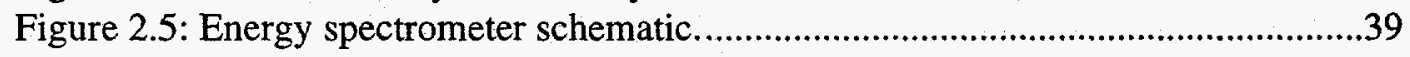

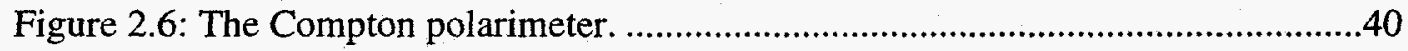

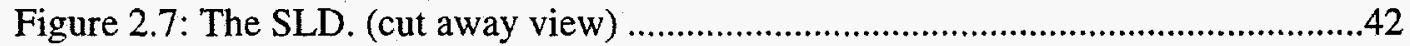

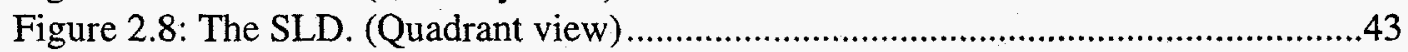

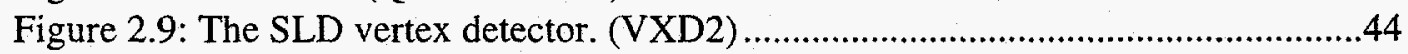

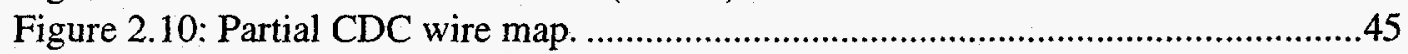

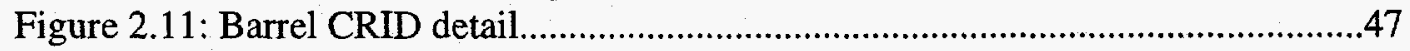

Figure 2.12: Typical barrel LAC modules...............................................................48

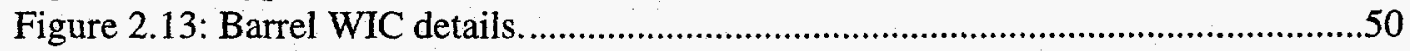

Figure 3.1: Overall trigger efficiency from tau Monte Carlo events. ............................56

Figure 3.2: Individual trigger efficiencies from tau Monte Carlo events. ......................57

Figure 3.3: Energy trigger efficiency estimate from correlations................................60

Figure 3.4: WAB trigger efficiency estimate from correlations...............................60

Figure 3.5: Track trigger efficiency estimates from correlations. ..................................61

Figure 3.6: Hadron trigger efficiency estimates from correlations.............................62

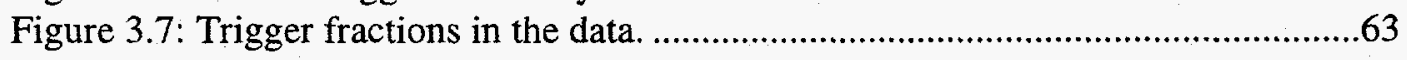

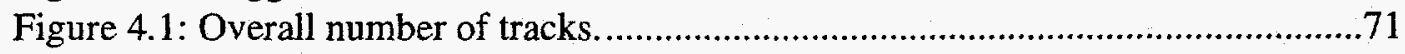

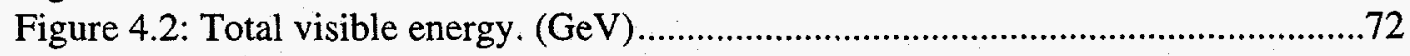

Figure 4.3: Number of unassociated clusters in jets. ................................................ 72

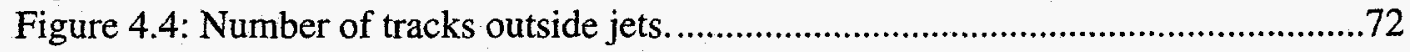

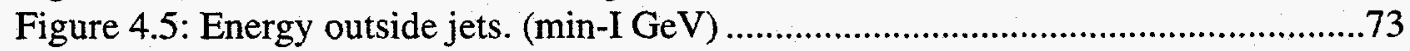

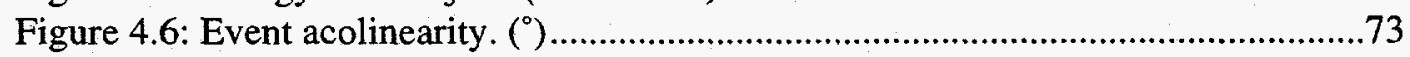

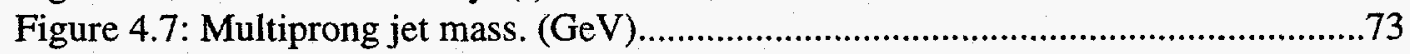

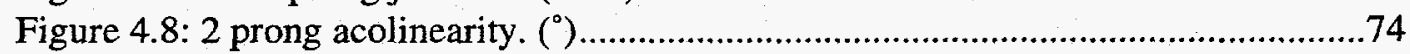

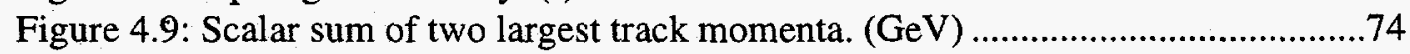

Figure 4.10: Total electromagnetic energy. (corrected $\mathrm{GeV}$ ) .....................................74

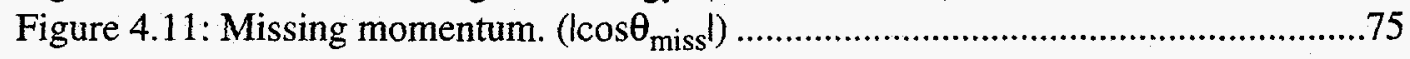




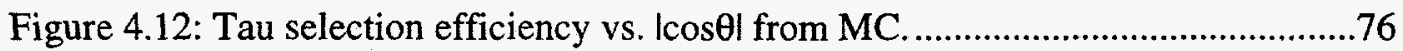

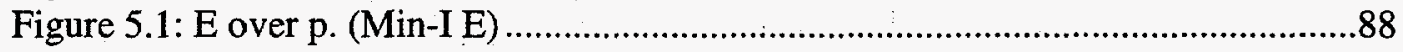

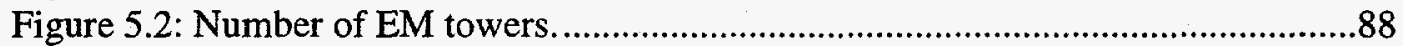

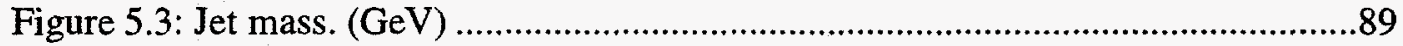

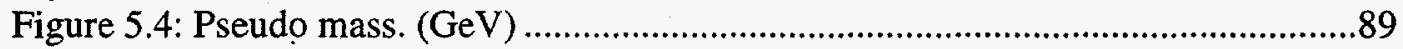

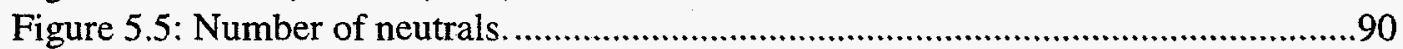

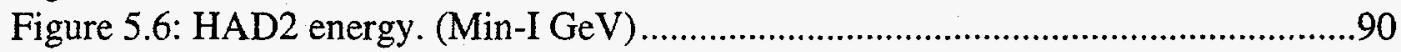

Figure 6.1: Electron radiative corrections vs. scaled energy..................................104

Figure 6.2: Muon radiative corrections vs. scaled energy. .......................................105

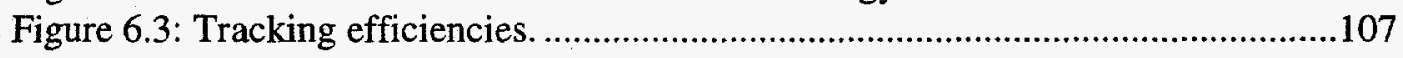

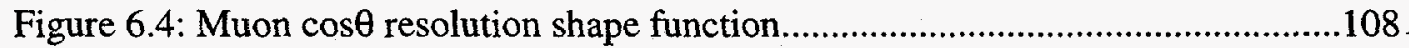

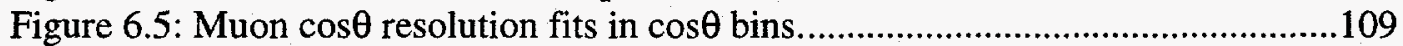

Figure 6.6: Muon $\cos \theta$ resolution fits in momentum bins......................................110

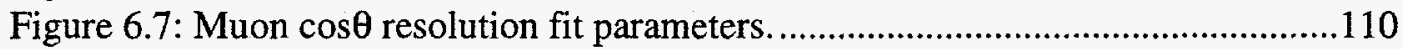

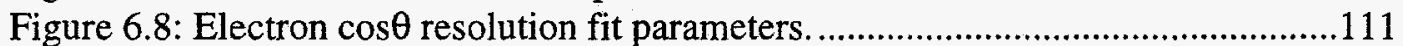

Figure 6.9: Muon scaled momentum resolution shape function...............................112

Figure 6.10: Muon momentum resolution fits in bins of $|\cos \theta|$.................................113

Figure 6.11: Muon momentum resolution fits in momentum bins............................113

Figure 6.12: Muon momentum resolution fit parameters. .......................................114

Figure 6.13: Electron momentum resolution shape fits in momentum bins................115

Figure 6.14: Electron resolution shape parameters..............................................116

Figure 6.15: Electron momentum resolution fits in momentum bins. .......................117

Figure 6.16: Electron momentum resolution fractions. ..........................................117

Figure 6.17: Electron momentum resolution in $|\cos \theta|$ bins. ......................................118

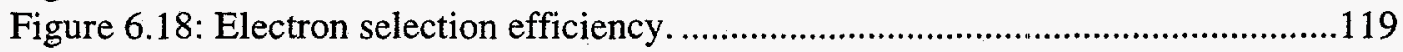

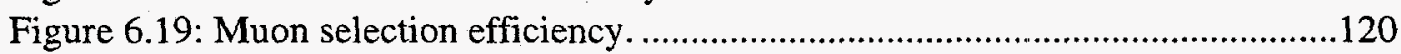

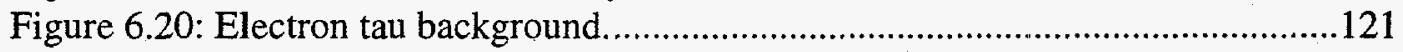

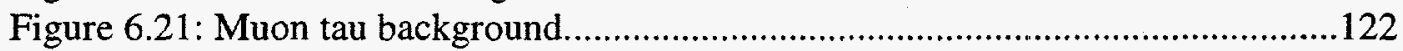

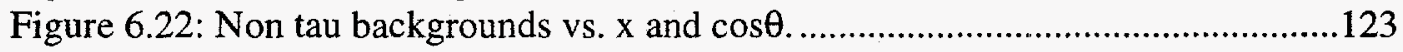

Figure 7.1: Data and Monte Carlo x spectra for left and right handed taus..................126

Figure 7.2: Fit results for 21 Monte Carlo samples. ...............................................134

Figure 7.3: Fit results for different subsamples of the data. ...................................136

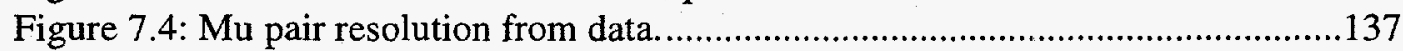

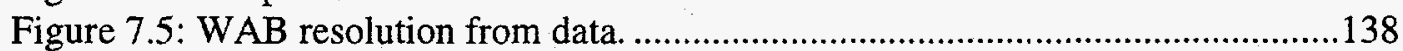

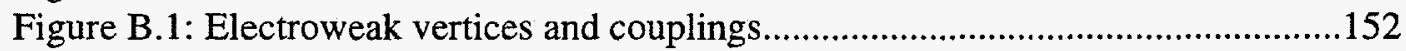

Figure E.1: EM LAC response for wide angle Bhabha events. (Min-I GeV) ..............162 


\section{List of Tables}

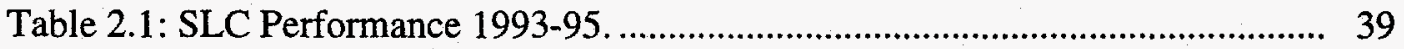

Table 3.1: Endcap Energy Trigger Rates for Various Decay Combinations.............. 58

Table 4.1: SLD Tau Sample Size.................................................................... 69

Table 4.2: Tau Selection Efficiency. (no Energy Trigger) ....................................... 76

Table 4.3: Tau Selection Efficiency. (with Energy Trigger) ................................... 77

Table 4.4: Tau Selection Efficiency by Topology from 94 Fall MC....................... 77

Table 4.5: Tau selection Efficiency for Events Including Given Decays.................. 77

Table 4.6: '94 Fall Monte Carlo Samples. ........................................................... 78

Table 4.7: Estimated Taus from Measured Luminosity.......................................... 80

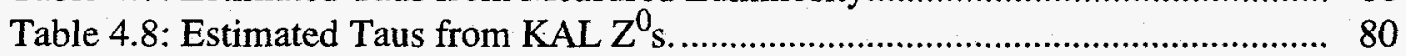

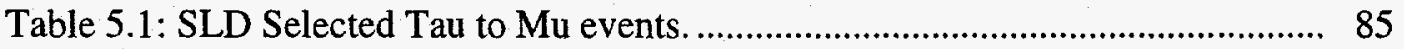

Table 5.2: SLD Selected Tau to Electron events................................................... 87

Table 5.3: Composition of MC Electronic Decay Sample. ...................................... 92

Table 5.4: Tau Electronic Branching Ratio Estimate. ….......................................... 92

Table 5.5: Composition of MC Muonic Decay Sample. .......................................... 93

Table 5.6: Tau Muonic Branching Ratio Estimate. …............................................. 94

Table 7.1: Electron Fit Results....................................................................... 127

Table 7.2: Electron Fit Correlation Coefficients................................................... 127

Table 7.3: Muon Fit Results............................................................................. 127

Table 7.4: Muon Fit Correlation Coefficients.................................................... 128

Table 7.5: Combined Fit with Variable Eta. ...................................................... 128

Table 7.6: Combined Fit with Fixed Eta............................................................... 129

Table 7.7: Combined Fit with Variable Eta Correlation Coefficients. .................... 129

Table 7.8: Combined Fit with Fixed Eta Correlation Coefficients.......................... 129

Table 7.9: Combined Fit with Variable Eta Systematics....................................... 131

Table 7.10: Combined Fit with Fixed Eta Systematics......................................... 132

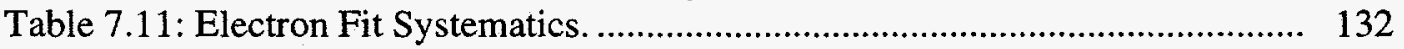

Table 7.12: Muon Fit Systematics. ................................................................. 133

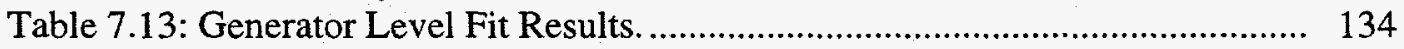

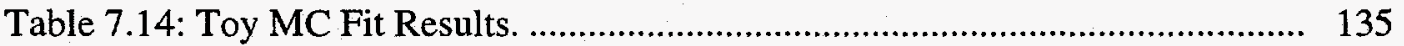

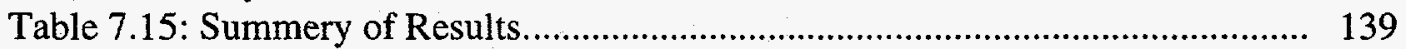

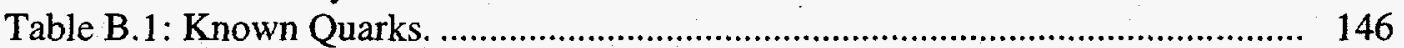

Table B.2: Known Leptons...................................................................... 146

Table B.3: Known Gauge Bosons................................................................... 146

Table B.4: Generations of Fermions................................................................. 147

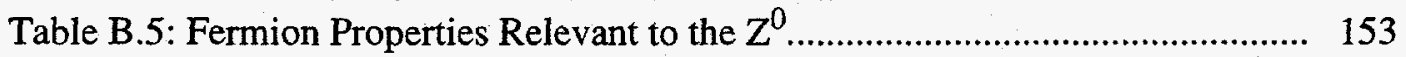

Table D.1: Leptonic $h_{\mathrm{V}}$ Results................................................................. 160

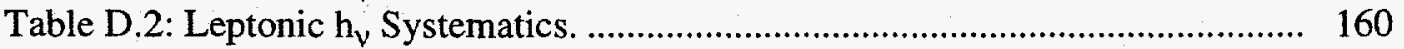




\section{Introduction}

The Michel parameters[1] describe the decay of a spin polarized heavy lepton into a lighter lepton. They were initially conceived to describe muon decay and are a complete parameterization of the momentum and angular momentum distributions of the electron resulting from polarized muon decay. This parameterization is easily generalized to leptonic tau decays. As such, the tau Michel parameters provide a test of lepton universality between taus and muons. In addition, if we measure the tau Michel parameters separately for tau decays to electrons and for tau decays to muons, we have a test of universality between electrons and muons. In the case of tau decay, it is difficult to measure the spin of the resulting lepton, so we confine ourselves to measuring its momentum spectrum. This reduces us to four measurable parameters for the tau decay to a muon and three for the tau decay to an electron, where the small electron mass relative to the tau suppresses the fourth parameter. In order to measure these parameters, we need a source of spin polarized taus, a way of measuring their spin, a way of identifying their decay products and a way of measuring the spectra of these decay products.

The SLAC ${ }^{1}$ Large Detector (SLD) at the SLAC Linear Collider (SLC) provides an excellent environment for measuring the tau Michel Parameters. Taus produced at the $Z^{0}$ resonance are naturally polarized, and this polarization is greatly enhanced by the large electron beam polarizations available at the SLC. In addition, the beam polarization dominates in determining the spins of the produced taus, so the tau polarization can be determined directly from the beam polarization and the tau production angle. This gives

1. Stanford Linear Accelerator Center. 
distinct advantages over the spin correlation techniques that are used in other tau Michel parameters measurements. Only one tau decay in a tau pair event need be identified, whereas correlation techniques require both to be identified. Also, the high SLC beam polarization provides much higher tau polarizations than are available at other facilities. In addition a single-tag measurement is a direct measurement of the spectrum, while a correlation technique measures the product of two spectra.

Due to the large mass of the $\mathrm{Z}^{0}$ boson, taus produced from real $\mathrm{Z}^{0}$ boson decay are highly boosted. As a result, at the SLC, it is relatively easy to select tau pair events and identify their decays. Tau pair events at the $Z^{0}$ resonance are characterized by two highly collimated low multiplicity jets and by missing momentum due to the neutrinos involved in the tau decays. This contrasts highly with high multiplicity hadronic $Z^{0}$ events and with high energy wide angle Bhabha and mu pair events, making for a high efficiency, low background tau sample. For the leptonic tau decays, we expect a single, isolated track in a tau hemisphere, making tracking and decay identification relatively simple. The SLD tracking system provides efficient muon identification over most of its useful tracking region, and electrons are easily identified by their shower shape and energy deposition in the calorimeter. In either case, the SLD provides a good track momentum measurement. Due to the high boost of the taus produced at the $Z^{0}$ resonance, the tau direction can also be easily determined from the thrust axis of the event. Thus, we can easily measure the energy and angular spectra of taus produced at the SLC.

This thesis describes a measurement of the Michel Parameters at SLD. We start off with a brief overview of tau production and decay in the Standard Model and a description of the Michel parameterization of leptonic tau decay spectra. Then we discuss the SLD and the SLC followed by a detailed discussion of the analysis, including event selection, fit techniques and corrections to the spectrum. Finally, we discuss the results. 


\section{Chapter 1}

\section{Physics Background}

Here, we give a brief overview of the physics of tau production at the $\mathrm{Z}^{0}$ resonance with polarized electrons, followed by a discussion of tau decay within the Standard Model and a description of the more general Michel parameterization and its relation to the Standard Model. A brief overview of the Standard Model and a more detailed description of the Electroweak physics relevant to this analysis is in Appendix B. We then discuss the transformation of the Michel parameterization to the lab frame for taus produced at the $\mathrm{Z}^{0}$ resonance, and finish with a discussion of the combined theoretical tau production and leptonic decay spectrum at the $Z^{0}$ resonance, and how we can use this to measure the tau Michel parameters.

\subsection{Tau Production at the $Z^{0}$ Resonance}

The tree level cross section for fermion pair production from electron-positron annihilation can be written as the sum of two diagrams, if the final state fermions are not electrons. Thus the cross section is proportional to $\left|\mathcal{M}_{Z}+\mathcal{M}_{\gamma}\right|^{2}$, where $\mathcal{M}_{Z}$ and $\mathcal{M}_{\gamma}$ are the matrix elements corresponding to the two diagrams. At the $Z^{0}$ resonance, the $Z^{0}$ exchange term dominates the photon term by a factor of $\sim 800$, and the gamma- $Z^{0}$ interference term is identically zero at the $Z^{0}$ pole, so to first order, we can discuss tau production at the $Z^{0}$ resonance in terms of the tree level $\mathrm{Z}^{0}$-exchange and incorporate the photon exchange and gamma- $Z^{0}$ interference terms into the radiative corrections to the theoretical production cross section. At the SLC, things are further complicated by the presence of beam polarization. 


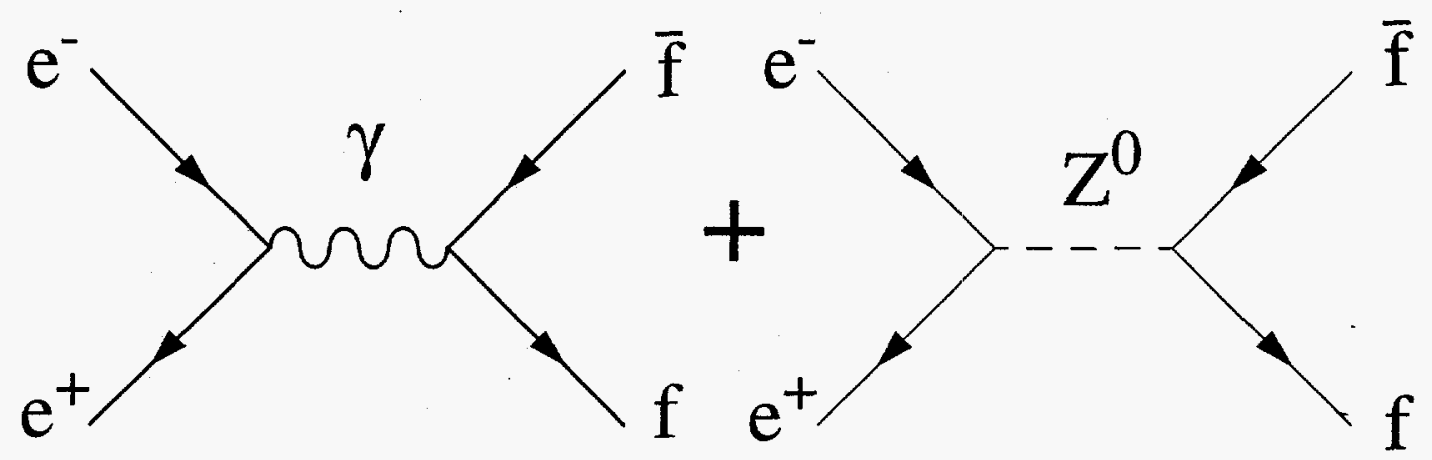

Figure 1.1: Tree level $\mathrm{e}^{+} \mathrm{e}^{-} \rightarrow \mathrm{f} \hat{\mathrm{f}}$ fermion production diagrams.

\subsubsection{Tree Level Fermion Pair Production Cross Sections}

Neglecting initial and final state polarization, we determine the differential cross section for fermion pair production at the $Z^{0}$ resonance $\left(\sqrt{s}=M_{Z}\right)$ to be

$$
\frac{1}{\sigma} \frac{\mathrm{d} \sigma}{\mathrm{dc}}=\frac{3}{8}\left[\left(1+\mathrm{c}^{2}\right)+2 \mathrm{~A}_{\mathrm{f}} \mathrm{A}_{\mathrm{e}} \mathrm{c}\right]
$$

where we have defined

$$
A_{f}=\frac{C_{V}^{f} C_{A}^{f}}{C_{V}^{f}{ }^{2}+C_{A}^{f}{ }^{2}}
$$

and $c=\cos \theta$, where $\theta$ is the polar angle of the outgoing fermion relative to the electron beam direction, and $\mathrm{C}_{\mathrm{V}}$ and $\mathrm{C}_{\mathrm{A}}$ are the $\mathrm{Z}^{0}$ couplings. The cross section is isotropic in azimuth and the momenta of the outgoing fermion and antifermion are equal and opposite with $\mathrm{E}_{\mathrm{f}}=\frac{1}{2} \mathrm{M}_{\mathrm{Z}}$, so the above expression completely describes the tree level production of fermion pairs from electron-positron annihilation at the $Z^{0}$ resonance. The second term gives rise to the forward backward cross section asymmetry used at LEP to measure the product $\mathrm{A}_{\mathrm{e}} \mathrm{A}_{\mathrm{f}}$ for various processes. Allowing for electron beam polarization, we get 


$$
\left.\frac{1}{\sigma} \frac{\mathrm{d} \sigma}{\mathrm{dc}}\right|_{\mathrm{P}_{\mathrm{e}}}=\frac{3}{8}\left[\left(1-\mathrm{A}_{\mathrm{e}} \mathrm{P}_{\mathrm{e}}\right)\left(1+\mathrm{c}^{2}\right)+2 \mathrm{~A}_{\mathrm{f}}\left(\mathrm{A}_{\mathrm{e}}-\mathrm{P}_{\mathrm{e}}\right) \mathrm{c}\right]
$$

Where $\mathrm{P}_{\mathrm{e}}$ is the longitudinal polarization of the incoming electron beam defined as

$$
P_{e}=\frac{n_{R}-n_{L}}{n_{R}+n_{L}}
$$

where $n_{R}$ and $n_{L}$ are the number of right and left-handed electrons in the incident electron beam. We assume that the positron polarization is negligible. Here we see the source of the left-right cross section asymmetry used by SLD to measure $A_{e}$. If we further look at lefthanded and right-handed final states, we get for the production of left-handed fermions

$$
\left.\frac{1}{\sigma} \frac{d \sigma_{L}}{d c}\right|_{P_{e}}=\frac{3}{16}\left(1+A_{f}\right)\left[\left(1-A_{e} P_{e}\right)\left(1+c^{2}\right)+2\left(A_{e}-P_{e}\right) c\right]
$$

and for right-handed fermions

$$
\left.\frac{1}{\sigma} \frac{d \sigma_{R}}{d c}\right|_{P_{e}}=\frac{3}{16}\left(1-A_{f}\right)\left[\left(1-A_{e} P_{e}\right)\left(1+c^{2}\right)-2\left(A_{e}-P_{e}\right) c\right]
$$

where in both cases the attendant antifermion has the opposite helicity due to angular momentum conservation in the decay of the spin one $Z^{0}$ and the fermion and antifermion opposite momenta. Note that for obvious reasons,

$$
\frac{1}{\sigma} \frac{\mathrm{d} \sigma}{\mathrm{dc}}=\frac{1}{\sigma} \frac{\mathrm{d} \sigma_{\mathrm{L}}}{\mathrm{dc}}+\frac{1}{\sigma} \frac{\mathrm{d} \sigma_{\mathrm{R}}}{\mathrm{dc}}
$$

\subsubsection{Final State Polarization}

We can define polarizations for the final state fermions as we did for the initial state electrons 


$$
P_{f}\left(c, P_{e}\right)=\frac{\frac{d \sigma_{R}}{d c}-\frac{d \sigma_{L}}{d c}}{\frac{d \sigma_{R}}{d c}+\frac{d \sigma_{L}}{d c}}
$$

This polarization is the polarization as measured along the produced fermion's direction of flight. The produced antifermion will, of course, have the opposite polarization. Substituting for the cross sections and simplifying the resulting expression gives

$$
P_{f}\left(c, P_{e}\right)=\frac{A_{f}+2 \frac{\left(A_{e}-P_{e}\right)}{\left(1-A_{e} P_{e}\right)} \frac{c}{\left(1+c^{2}\right)}}{1+2 A_{f} \frac{\left(A_{e}-P_{e}\right)}{\left(1-A_{e} P_{e}\right)} \frac{c}{\left(1+c^{2}\right)}}
$$

For the tau, we need only use the correct values of $\mathrm{A}_{e}$ and $\mathrm{A}_{\tau}$ to get the tree level cross sections and polarizations. [2][3]

\subsection{Tau Decay in the Standard Model}

In the Standard Model, we expect taus to decay via a virtual $W$ to a tau neutrino and whatever the W system decays to.

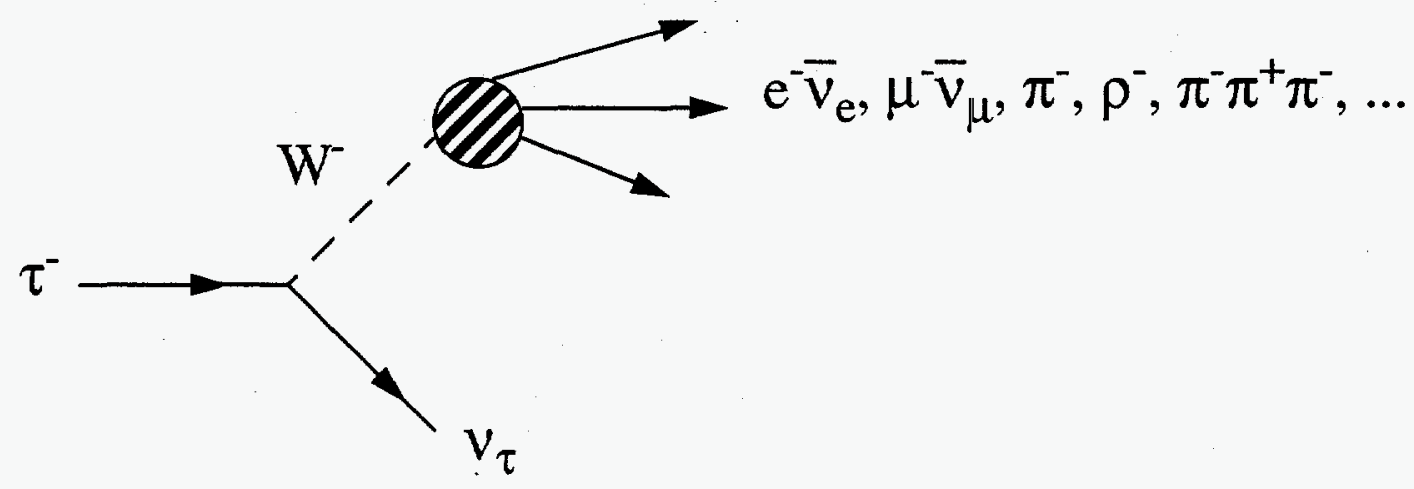

Figure 1.2: Generic tau decay.

Thus we expect that tau decay will be a V-A interaction other than any QCD effects that 
may occur in hadronic decays. In this analysis, we are studying tau decays to leptons, in which case the tree-level diagram for the decay is quite simple.

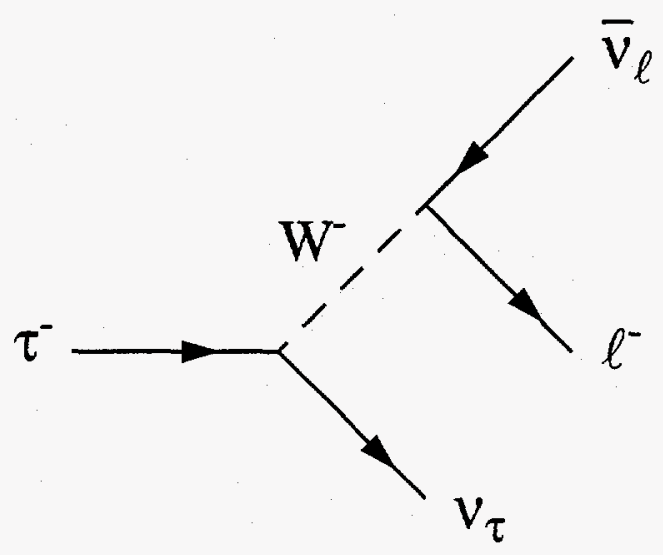

Figure 1.3: Leptonic tau decay.

This diagram can be evaluated in the Standard Model to give the differential decay rate for a tau at rest decaying to a charged lepton and two neutrinos.[4]

$$
\begin{aligned}
\mathrm{d} \Gamma \propto \mathrm{d}^{3} \mathrm{p}_{\ell}\left[\left[\left(\mathrm{m}_{\tau}-\mathrm{E}_{\ell}\right)^{2}-\mathrm{p}_{\ell}^{2}\right]\left[\mathrm{E}_{\ell}+\mathbf{s}_{\tau} \bullet \mathbf{p}_{\ell}\right]\right. \\
\left.\quad+2\left[\mathrm{~m}_{\tau}-\mathrm{E}_{\ell}-\mathbf{s}_{\tau} \bullet \mathbf{p}_{\ell}\right]\left[\left(\mathrm{m}_{\tau}-\mathrm{E}_{\ell}\right) \mathrm{E}_{\ell}+\mathrm{p}_{\ell}^{2}\right]\right]
\end{aligned}
$$

Where we have integrated over the neutrinos' momenta and summed over the final state spins and assumed that the $\mathrm{W}$ mass is much larger than the momentum transfer. In the decay, the kinematics require that $\mathrm{E}_{\ell}<\mathrm{m}_{\tau} / 2$. We can simplify (1.10) by making the approximation that the outgoing lepton is highly relativistic $\left(\mathrm{p}_{\ell} \approx \mathrm{E}_{\ell}\right)$ which holds if $\mathrm{m}_{\ell}^{2} \ll \mathrm{m}_{\tau}^{2}$

$$
\mathrm{d} \Gamma \propto \mathrm{E}_{\ell} \mathrm{dE} \mathrm{E}_{\ell} \mathrm{d} \Omega\left[3 \mathrm{~m}_{\tau}^{2} \mathrm{E}_{\ell}-4 \mathrm{~m}_{\tau} \mathrm{E}_{\ell}^{2}+\mathrm{m}_{\tau}^{2} \mathrm{E}_{\ell}\left(\mathbf{s}_{\tau} \bullet \hat{\mathbf{p}}_{\ell}\right)-4 \mathrm{~m}_{\tau} \mathrm{E}_{\ell}^{2}\left(\mathbf{s}_{\tau} \bullet \hat{\mathbf{p}_{\ell}}\right)\right]
$$

We can then rewrite this by defining $\mathrm{x}=2 \mathrm{E}_{\ell} / \mathrm{m}_{\tau}$ and $\cos \theta=\mathbf{s}_{\tau} \bullet \hat{\mathbf{p}}_{\ell}$ to get 


$$
\frac{d^{2} \Gamma}{d x d \Omega} \propto x^{2}[3-2 x-(2 x-1) \cos \theta]
$$

The spectrum is isotropic in the azimuthal angle of the outgoing lepton as one would expect from the symmetries in the decay. This is the expected Standard Model spectrum for the lepton from a tau decay. To get the spectrum for $\tau^{+}$decay, we need to reverse the sign of the polarization terms. The $\mathrm{W}$ couples only to left-handed particles and righthanded antiparticles. As a result, the spin dependence of anti-tau $\left(\tau^{+}\right)$decay is reversed. We can accomplish this in the above spectrum by reversing the sign of the $\cos \theta$ term.[5]

\subsection{More General Parameterizations for Leptonic Tau Decays}

Long before the development of the electroweak Standard Model or even the V-A theory of charged-current weak interactions, physicists were faced with the problem of muon decay. The muon decays to an electron and two neutrinos, and the physics of this decay should be identical to that of leptonic tau decay other than effects of the masses of the particles. A general parameterization for the electron spectrum from muon decay was developed. We can apply this parameterization unchanged to the problem of leptonic tau decay. We can also develop a parameterization for leptonic tau decays in terms of a most general formulation of the weak interaction (See Appendix B).

\subsubsection{The Michel Parameterization}

We can parameterize the complete electron spin and momentum spectrum from muon decay as

$$
\frac{\mathrm{d}^{2} \Gamma}{\mathrm{dxd} \Omega} \propto \sqrt{\mathrm{x}^{2}-\mathrm{x}_{0}^{2}}\left[\mathrm{~F}_{\mathrm{IS}}(\mathrm{x})-\mathrm{P}_{\mu} \cos \theta \mathrm{F}_{\mathrm{AS}}(\mathrm{x})\right]\left[1+\mathbf{P}_{\mathrm{e}}(\mathrm{x}, \theta) \bullet \hat{\zeta}\right]
$$

where we define 


$$
E_{e}^{\max }=\frac{m_{\mu}^{2}+m_{e}^{2}}{2 m_{\mu}}, x=\frac{E_{e}}{E_{e}^{\max }}, \text { and } x_{0}=\frac{m_{e}}{E_{e}^{\max }} \text {. }
$$

and $\hat{\zeta}$ is the direction of the initial muon polarization. As in the case of tau decay, the spectrum for $\mu^{+}$decay can be defined by reversing the sign of the muon polarization dependant terms. In principal, we could define a separate set of parameters to describe the $\mu^{+}$decay, but we expect the two spectra to be identical other than the reverse of the polarization terms. The functions $F_{I S}, F_{A S}$ and $\mathbf{P}_{\mathrm{e}}$ are defined as follows

$$
\begin{gathered}
\mathrm{F}_{\mathrm{IS}}(\mathrm{x})=\mathrm{x}(1-\mathrm{x})+\frac{2}{9} \rho\left(4 \mathrm{x}^{2}-3 \mathrm{x}-\mathrm{x}_{0}^{2}\right)+\eta \mathrm{x}_{0}(1-\mathrm{x}) \\
\mathrm{F}_{\mathrm{AS}}(\mathrm{x})=\frac{1}{3} \xi \sqrt{\mathrm{x}^{2}-\mathrm{x}_{0}^{2}}\left[1-\mathrm{x}+\frac{2}{3} \delta\left(4 \mathrm{x}-3-\left(\sqrt{1-\mathrm{x}_{0}^{2}}-1\right)\right)\right] \\
\mathbf{P}_{\mathrm{e}}(\mathrm{x}, \theta)=\mathrm{P}_{\mathrm{T}_{1}}(\mathrm{x}, \theta) \hat{\mathrm{x}}+\mathrm{P}_{\mathrm{T}_{2}}(\mathrm{x}, \theta) \hat{\mathrm{y}}+\mathrm{P}_{\mathrm{L}}(\mathrm{x}, \theta) \hat{\mathrm{z}}
\end{gathered}
$$

where $\hat{z}$ is parallel to the electron momentum, $\hat{y}$ is parallel to $\hat{z} \times \mathbf{P}_{\mu}$, and $\hat{x}=\hat{y} \times \hat{z}$, and the components of $\mathbf{P}_{\mathrm{e}}$ are

$$
\begin{aligned}
& \mathrm{P}_{\mathrm{T}_{1}}(\mathrm{x}, \theta)=\frac{\mathrm{P}_{\mu} \sin \theta \mathrm{F}_{\mathrm{T}_{1}}(\mathrm{x})}{\mathrm{F}_{\mathrm{IS}}(\mathrm{x})-\mathrm{P}_{\mu} \cos \theta \mathrm{F}_{\mathrm{AS}}(\mathrm{x})} \\
& \mathrm{P}_{\mathrm{T}_{2}}(\mathrm{x}, \theta)=\frac{\mathrm{P}_{\mu} \sin \theta \mathrm{F}_{\mathrm{T}_{2}}(\mathrm{x})}{\mathrm{F}_{\mathrm{IS}}(\mathrm{x})-\mathrm{P}_{\mu} \cos \theta \mathrm{F}_{\mathrm{AS}}(\mathrm{x})} \text {, and } \\
& P_{L}(x, \theta)=-F_{I P}(x)+\frac{P_{\mu} \cos \theta F_{A P}(x)}{F_{I S}(x)-P_{\mu} \cos \theta F_{A S}(x)} \text {, where } \\
& \mathrm{F}_{\mathrm{T}_{1}}(\mathrm{x})=\frac{1}{12}\left(-2\left[\xi^{\prime \prime}+12\left(\rho-\frac{3}{4}\right)\right](1-\mathrm{x}) \mathrm{x}_{0},\right. \\
& \left.-3 \eta\left(x^{2}-x_{0}^{2}\right)+\eta "\left(-3 x^{2}+4 x-x_{0}^{2}\right)\right)
\end{aligned}
$$




$$
\begin{aligned}
\mathrm{F}_{\mathrm{T}_{2}}(\mathrm{x})= & \frac{1}{3} \sqrt{\mathrm{x}^{2}-\mathrm{x}_{0}^{2}}\left[3 \frac{\alpha^{\prime}}{\mathrm{A}}(1-\mathrm{x})+2 \frac{\beta^{\prime}}{\mathrm{A}} \sqrt{1-\mathrm{x}_{0}^{2}}\right] \\
\mathrm{F}_{\mathrm{IP}}(\mathrm{x})= & \frac{1}{54} \sqrt{\mathrm{x}^{2}-\mathrm{x}_{0}^{2}}\left[9 \xi^{\prime}\left(-2 \mathrm{x}+2+\sqrt{1-\mathrm{x}_{0}^{2}}\right)\right. \text {, and } \\
& \left.+4 \xi\left(\delta-\frac{3}{4}\right)\left(4 \mathrm{x}-4+\sqrt{1-\mathrm{x}_{0}^{2}}\right)\right] \\
\mathrm{F}_{\mathrm{AP}}(\mathrm{x})= & \frac{1}{6}\left[\xi^{\prime \prime}\left(2 \mathrm{x}^{2}-\mathrm{x}-\mathrm{x}_{0}^{2}\right)+4\left(\rho-\frac{3}{4}\right)\left(4 \mathrm{x}^{2}-3 \mathrm{x}-\mathrm{x}_{0}^{2}\right)\right. \\
& \left.+2 \eta^{\prime \prime}(1-\mathrm{x}) \mathrm{x}_{0}\right]
\end{aligned}
$$

Thus the entire decay spectrum is parameterized by the parameters $\rho, \xi, \xi^{\prime}, \xi^{\prime \prime}, \delta, \eta, \eta ", \alpha J$ $A, \alpha^{\prime} / A, \beta / A$, and $\beta^{\prime} / A .[6]$ If we neglect terms quadratic in the electron mass and look only at the momentum spectrum of the outgoing electron, we get the more familiar Michel spectrum

$$
\begin{gathered}
\frac{\mathrm{d}^{2} \Gamma}{\mathrm{dxd} \Omega} \propto \mathrm{x}^{2}\left\{3(1-\mathrm{x})+\frac{2}{3} \mathrm{p}(4 \mathrm{x}-3)+6 \eta \mathrm{x}_{0}\left(\frac{1-\mathrm{x}}{\mathrm{x}}\right)\right. \\
\left.-\mathrm{P}_{\mu} \xi \cos \theta\left[1-\mathrm{x}+\frac{2}{3} \delta(4 \mathrm{x}-3)\right]\right\}
\end{gathered}
$$

Where the momentum spectrum is now parameterized by the four Michel Parameters $\rho, \xi$, $\delta$ and $\eta$, and $x$ is now defined as $2 \mathrm{E}_{\mathrm{e}} / \mathrm{m}_{\mu}$. This formalism holds equally well for leptonic tau decays. We measure the product $\xi \delta$ instead of $\delta$ as $\delta$ only enters into the parameterization as a part of that product, and the parameters as defined have some correlations, which are not exacerbated by using the product. If we compare this spectrum with the Standard Model spectrum (1.12), we see that for the Standard Model, we expect $\rho=\frac{3}{4}, \xi=1$, $\delta=\frac{3}{4}$, and $\eta=0$. A more general parameterization is discussed in Appendix B.

\subsection{Lab Frame Decay Spectrum}

As we have seen, at the $\mathrm{Z}^{0}$ resonance, taus are not produced at rest. They are produced 
highly relativistically and, as a result, their decay products are highly collimated along the tau flight direction. Due to the large boost of the taus produced at the $\mathrm{Z}^{0}$ resonance, for leptonic tau decays, the only quantity we can measure well is the boosted energy of the resultant lepton. Thus to get the theoretical spectrum corresponding to our observations, we must boost the tau decay spectrum to the lab frame and integrate over the decay angle. [2]

\subsubsection{The Boost}

Starting with the tau rest frame Michel spectrum in $\mathrm{x}=2 \mathrm{E}_{\ell}^{\mathrm{rest}} / \mathrm{m}_{\tau}$, where for $\tau^{+} \mathrm{s}$ we reverse the sign of the $P_{\tau}$ term,

$$
\begin{aligned}
\frac{\mathrm{d}^{2} \Gamma}{\mathrm{dxd}(\cos \theta)} \propto & \mathrm{x}^{2}\left\{3(1-\mathrm{x})+\frac{2}{3} \rho(4 \mathrm{x}-3)+6 \eta \frac{\mathrm{m}_{\ell}}{\mathrm{m}_{\tau}}\left(\frac{1-\mathrm{x}}{\mathrm{x}}\right),\right. \\
& \left.-\mathrm{P}_{\tau} \xi \cos \theta\left[1-\mathrm{x}+\frac{2}{3} \delta(4 \mathrm{x}-3)\right]\right\}
\end{aligned}
$$

we boost along the direction of polarization so that the tau has energy $E_{\text {beam }}$. At the $Z^{0}$ resonance, as we have seen, taus are produced longitudinally polarized. We choose the axes so that the decay is in the $\mathrm{x}-\mathrm{z}$ plane and the tau flight direction is along the $\mathrm{z}$-axis. For this transformation, $\gamma=E_{\text {beam }} / m_{\tau}$, and $\beta \gamma=\sqrt{E_{\text {beam }}^{2} / m_{\tau}^{2}-1}$. This gives us for the outgoing lepton,

$$
\left[\begin{array}{cccc}
\gamma & 0 & 0 & \beta \gamma \\
0 & 1 & 0 & 0 \\
0 & 0 & 1 & 0 \\
\beta \gamma & 0 & 0 & \gamma
\end{array}\right]\left[\begin{array}{c}
\mathrm{m}_{\tau} \\
\frac{2}{p_{\ell}} \sin \theta \\
0 \\
p_{\ell} \cos \theta
\end{array}\right]=\left[\begin{array}{c}
\gamma \frac{m_{\tau}}{2} x+\beta \gamma p_{\ell} \cos \theta \\
p_{1} \sin \theta \\
0 \\
m_{\tau} \frac{\tau}{2} x+\gamma p_{\ell} \cos \theta
\end{array}\right]
$$

where $\mathrm{p}_{\ell}=\sqrt{\mathrm{x}^{2} \mathrm{~m}_{\tau}^{2} / 4-\mathrm{m}_{\ell}^{2}}$. If we define 


$$
\mathrm{y} \equiv \frac{\mathrm{E}_{\ell}^{\text {boost }}}{\mathrm{E}_{\text {beam }}}=\frac{\mathrm{E}_{\text {beam } \frac{\mathrm{x}}{2}}+\sqrt{\left(\mathrm{E}_{\text {beam }}^{2}-\mathrm{m}_{\tau}^{2}\right)\left(\frac{\mathrm{x}^{2}}{4}-\frac{\mathrm{m}_{\ell}^{2}}{\mathrm{~m}_{\tau}^{2}}\right)} \cos \theta}{\mathrm{E}_{\text {beam }}}
$$

we can make the approximations that $\mathrm{m}_{\tau}^{2} \ll \mathrm{E}_{\text {beam }}^{2}$, and $\mathrm{m}_{\ell}^{2} \ll \mathrm{m}_{\tau}^{2}$, to get

$$
y=\frac{x}{2}+\frac{x}{2} \cos \theta
$$

For taus produced at the SLC, we have $\mathrm{E}_{\text {beam }}=45.63 \mathrm{GeV}$, compared to $\mathrm{m}_{\tau}=1.777 \mathrm{GeV}$, and for the decay leptons, $m_{e}=0.5110 \mathrm{MeV}$ and $m_{\mu}=105.7 \mathrm{MeV}$, so these approximations all hold to the $0.5 \%$ level.[7]

\subsubsection{Applying the Boost}

We can now make the change of variables

$$
\cos \theta=\frac{2 y}{x}-1
$$

This gives us

$$
\begin{aligned}
\frac{\mathrm{d}^{2} \Gamma}{\mathrm{dxdy}} \propto & 2 \mathrm{x}\left\{3(1-\mathrm{x})+\frac{2}{3} \rho(4 \mathrm{x}-3)+6 \eta \frac{\mathrm{m}_{\ell}}{\mathrm{m}_{\tau}}\left(\frac{1-\mathrm{x}}{\mathrm{x}}\right)\right. \\
& \left.-\mathrm{P}_{\tau} \xi\left(\frac{2 \mathrm{y}}{\mathrm{x}}-1\right)\left[1-\mathrm{x}+\frac{2}{3} \delta(4 \mathrm{x}-3)\right]\right\}
\end{aligned}
$$

Integrating this over $\mathrm{x}$ from $\mathrm{y}$ to 1 , which is its allowed kinematic range, gives us the final boosted spectrum 


$$
\begin{aligned}
\frac{1}{\Gamma} \frac{\mathrm{d} \Gamma}{\mathrm{dy}}=\frac{1}{\Gamma} \int_{\mathrm{y}}^{1} \frac{\mathrm{d}^{2} \Gamma}{\mathrm{dxdy} d x=} & 2-6 \mathrm{y}^{2}+4 \mathrm{y}^{3} \\
& +\rho\left(-\frac{4}{9}+4 \mathrm{y}^{2}-\frac{32}{9} \mathrm{y}^{3}\right) \\
& +\eta \frac{\mathrm{m}_{\ell}}{\mathrm{m}_{\tau}}\left(12-24 \mathrm{y}+12 \mathrm{y}^{2}\right) \\
& +\mathrm{P}_{\tau} \xi\left(-\frac{2}{3}+4 \mathrm{y}-6 \mathrm{y}^{2}+\frac{8}{3} \mathrm{y}^{3}\right) \\
& +\mathrm{P}_{\tau} \xi \delta\left(\frac{4}{9}-\frac{16}{3} \mathrm{y}+12 \mathrm{y}^{2}-\frac{64}{9} \mathrm{y}^{3}\right)
\end{aligned}
$$

where we have also normalized the distribution so that it integrates to one over the full allowed range of $y$. The $\tau^{+}$spectrum is still obtained by reversing the sign of $P_{\tau}$.

\subsubsection{Some Notation}

We now introduce some notation. We redefine $\mathrm{x}=\mathrm{E}_{\ell}^{\mathrm{lab}} / \mathrm{E}_{\mathrm{beam}}=\mathrm{y}$, and define some functions for the various terms in the spectrum.

$$
\begin{aligned}
\frac{1}{\Gamma} \frac{d \Gamma}{d x} & =f_{c}(x)+\rho f_{\rho}(x)+\eta f_{\eta}^{\ell}(x)+P_{\tau} \xi g_{\xi}(x)+P_{\tau} \xi \delta g_{\xi \delta}(x) \\
& =f(x)+P_{\tau} g(x)
\end{aligned}
$$

The fs and gs may be inferred from (1.23). We can also write the decay spectra for left and right-handed taus. For left-handed taus, we have $P_{\tau}=-1$ and for the right-handed, $P_{\tau}=1$.

$$
\begin{aligned}
& \frac{1}{\Gamma} \frac{d \Gamma_{L}}{d x}=f(x)-g(x), \text { and } \\
& \frac{1}{\Gamma} \frac{d \Gamma_{R}}{d x}=f(x)+g(x)
\end{aligned}
$$

In this case for the $\tau^{+}$spectrum, we reverse the sign of the $\mathrm{g}$ term. 


\subsection{Combined Production and Decay Spectrum}

We now need to combine the various parts that we have developed into a complete description of the lepton momentum spectrum for leptons from tau decay, where the taus are produced at the $Z^{0}$ resonance with polarized beams. We can write the combined spectrum as

$$
\begin{aligned}
& \frac{1}{\sigma} \frac{\mathrm{d}^{2} \sigma}{\mathrm{dxd}(\cos \theta)}\left(\mathrm{x}, \cos \theta, \mathrm{P}_{\mathrm{e}}\right) \propto \\
& W\left(\mathrm{x}, \cos \theta, \mathrm{P}_{\mathrm{e}}\right) \equiv \frac{1}{\sigma} \frac{\mathrm{d} \sigma_{\mathrm{L}}}{\mathrm{d}(\cos \theta)} \frac{1}{\Gamma} \frac{\mathrm{d} \Gamma_{\mathrm{L}}}{\mathrm{dx}}+\frac{1}{\sigma} \frac{\mathrm{d} \sigma_{\mathrm{R}}}{\mathrm{d}(\cos \theta)} \frac{1}{\Gamma} \frac{\mathrm{d} \Gamma_{\mathrm{R}}}{\mathrm{dx}}
\end{aligned}
$$

Substituting the decay spectra and defining $c=\cos \theta$, the cosine of the polar angle of the tau relative to the incident electron beam gives

$$
W=\frac{1}{\sigma} \frac{d \sigma_{L}}{d c}(f(x)-g(x))+\frac{1}{\sigma} \frac{d \sigma_{R}}{d c}(f(x)+g(x))
$$

which can be rearranged to give

$$
W=\frac{1}{\sigma}\left(\frac{d \sigma_{L}}{d c}+\frac{d \sigma_{R}}{d c}\right)\left[f(x)+\left(\frac{\frac{d \sigma_{R}}{d c}-\frac{d \sigma_{L}}{d c}}{\frac{d \sigma_{L}}{d c}+\frac{d \sigma_{R}}{d c}}\right) g(x)\right]
$$

which, by substituting previously defined quantities gives,

$$
W\left(x, c, P_{e}\right)=\frac{1}{\sigma} \frac{d \sigma}{d c}\left(P_{e}, c\right)\left[f(x)+P_{\tau}\left(P_{e}, c\right) g(x)\right]
$$

which is the theoretical spectrum we are trying to measure. Here, we are fortunate in that if we take $\cos \theta$ to be the polar angle of the $\tau^{-}$in the event, we get identical spectra for $\tau^{+}$ and $\tau^{-}$decays due to the fact that for the $\tau^{+}$, both $\mathrm{P}_{\tau}$ and $\mathrm{g}$ change sign. In this form, we clearly see that high tau polarizations will enhance measurements of the $g$ terms of the 
spectrum. Plotting $P_{\tau}\left(P_{e}, c\right)$, using $A_{e}=A_{\tau}=0.15$ (The current SLD value is $A_{e}=$ $0.1542 \pm 0.0039$. We expect $A_{e}$ and $A_{\tau}$ to be equal due to lepton universality.) and for several common beam polarizations, we get Fig. 1.4.

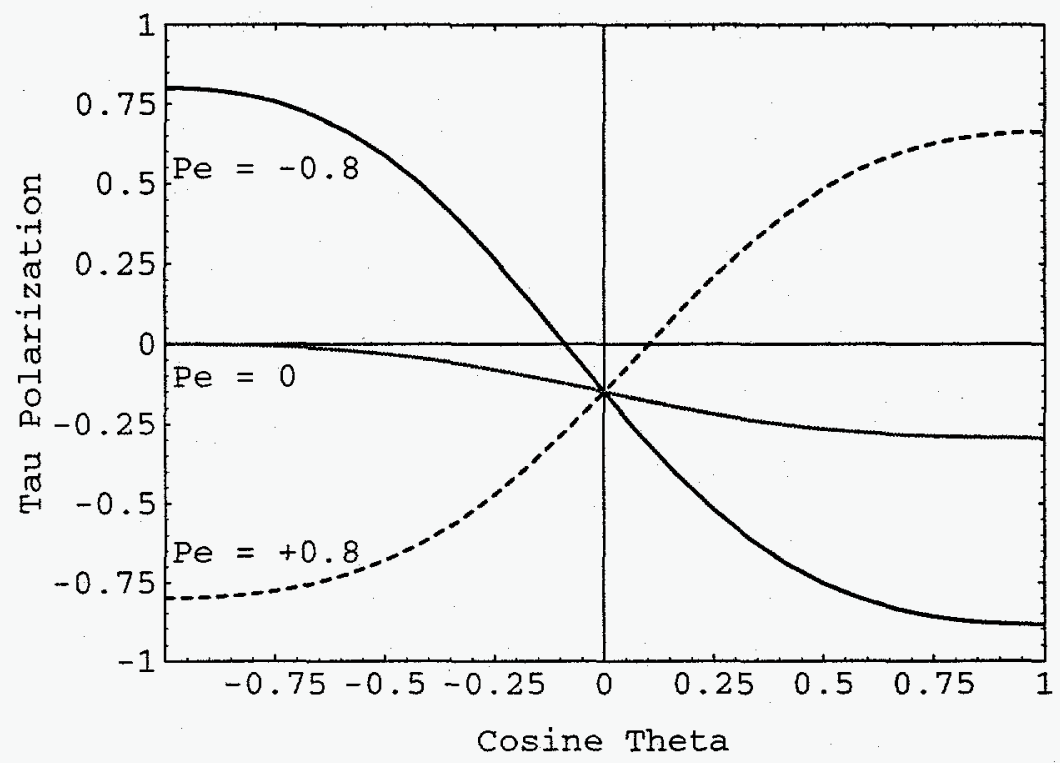

Figure 1.4: Tau Polarization vs. $\cos \theta$ for various beam polarizations.

From this, it is clear that high beam polarizations are essential to get high tau polarizations. Also, we see that at the SLC, especially at high $\cos \theta$, the tau polarization is largely determined by the beam polarization. At lower energies, the situation is worse than that at LEP as the $\mathrm{Z}^{0}$ term in the tau production cross section is not significant, and without beam polarization, the tree level final state fermion polarization is zero. At LEP and at the lower energy experiments, spin correlations between the two taus produced in a tau pair event are used to make the measurement of the $\mathrm{g}$ terms of the Michel spectrum.

Plotting the momentum spectral functions gives us Fig. 1.5 and Fig. 1.6. The constant term is the part of the spectrum that is not multiplied by a Michel parameter. The $\eta$ term is plotted for the muon mass, since it would be heavily suppressed by the small electron 
mass and essentially be zero. The $\rho, \xi$ and $\xi \delta$ terms all integrate to zero, while the $\eta$ term adds to the spectrum.

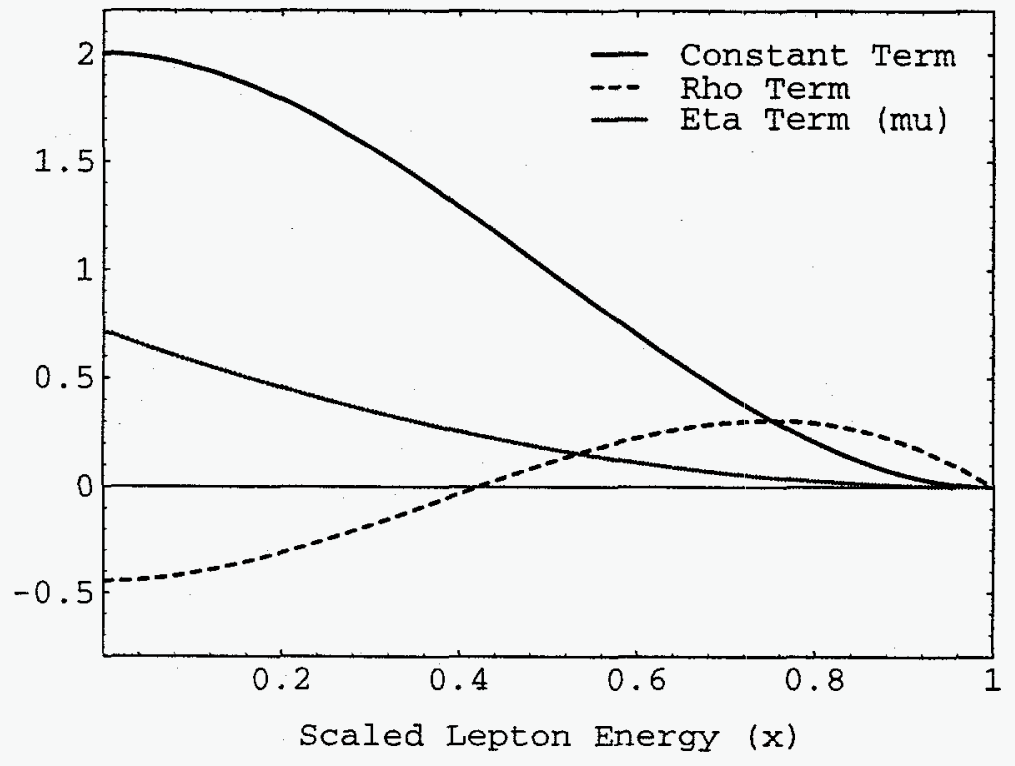

Figure 1.5: Polarization independent spectral functions.

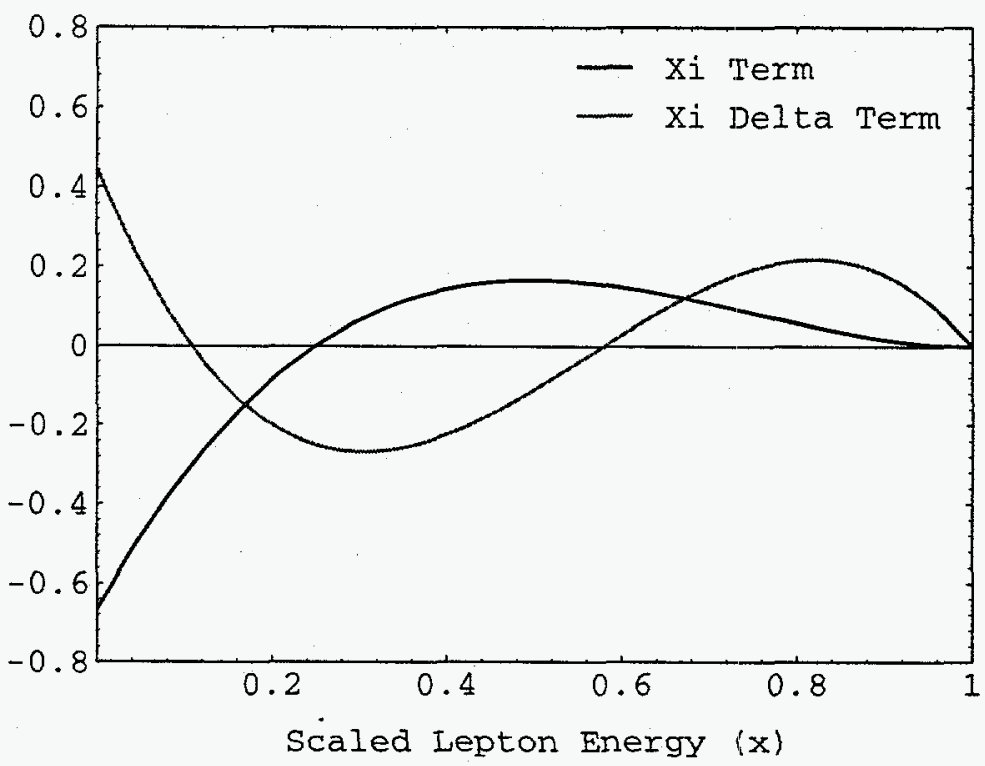

Figure 1.6: Polarization dependant spectral functions. 
Using the Standard Model values for the Michel parameters, we can calculate the expected spectra for left and right-handed leptonic tau decay (Fig. 1.7). We can see that there is a clear difference between the lepton spectrum from left-handed tau decay and that from right-handed tau decay. Measuring this difference will give us the polarization dependant Michel parameters $\xi$ and $\xi \delta$, and measuring the overall shape of the spectrum will give us $\rho$ and $\eta$.

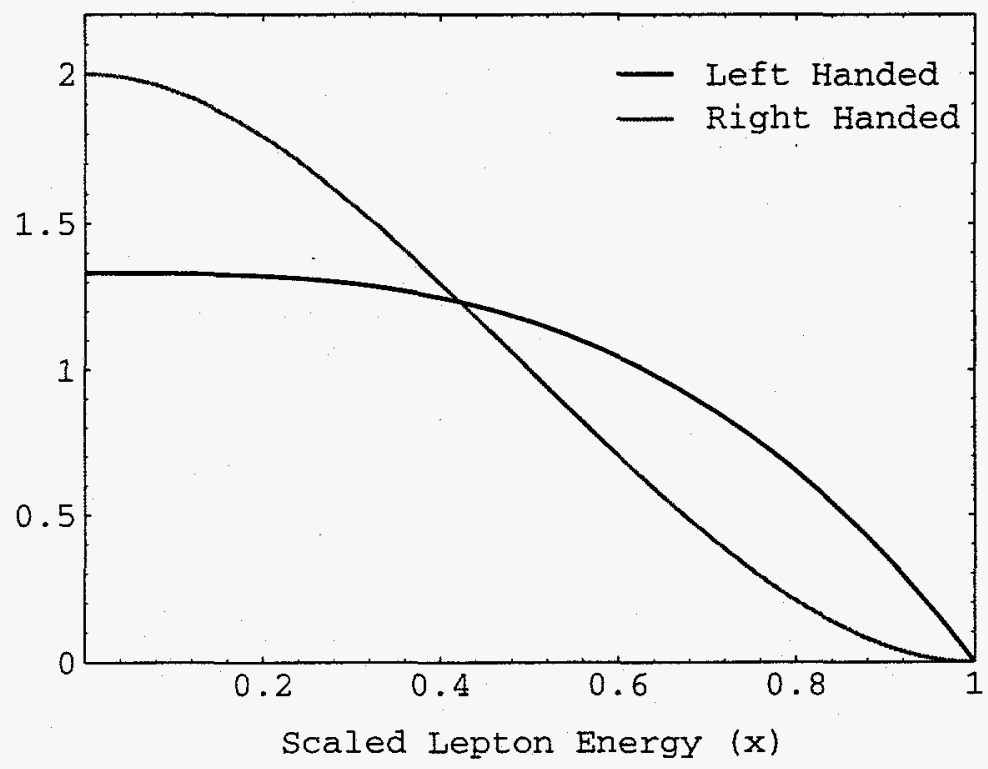

Figure 1.7: Left and right-handed lab frame tau Michel spectra.

\subsection{Performing the Measurement}

As we have seen, the Michel parameters describe the decay of spin polarized lepton to a lighter lepton and two neutrinos. To measure this for the tau, we need a source of spin polarized taus and a way measuring their decay spectra. Taus are produced naturally polarized at the $Z^{0}$ resonance. At the SLC, this is enhanced by the presence of polarized electrons. As a result, we can use the known form of the tau polarization distribution to tag the spin of the produced taus. At the $Z^{0}$ resonance, the taus are highly boosted and their decay 
products are highly collimated as a result. Due to this, we lose some of the rest frame decay angle information. Fortunately, the boosted energy spectrum contains enough information to allow us to measure the four Michel parameters that describe the decay lepton's momentum spectrum. It is also possible to do this measurement utilizing the spin correlations between the tau and anti-tau produced in each event. This method is restricted by the additional requirement of identifying both decays in a tau pair and since it measures the product of the two distributions, it can only measure the magnitude of the spin dependant terms of the spectrum and not their sign. Thus a single tag measurement of the tau Michel parameters at the SLD will provide a good complementary measurement to the spin correlation measurements made by the LEP experiments, CLEO and Argus.[7][8]-[11] 


\section{Chapter 2}

\section{The SLC and the SLD}

Here we describe the polarized $S_{L C}{ }^{1}$ and the $\operatorname{SLD}^{2}$. The data used in this analysis were collected at the SLD during the 1993 and $1994-5$ polarized SLC runs. During the 1993 run, the SLC provided us with $50 \mathrm{k} \mathrm{Z} \mathrm{Z}^{0}$ bosons with $63 \%$ electron beam polarization, and during $1994-95$, it provided us with $100 \mathrm{k} \mathrm{Z}^{0}$ bosons with $77.2 \%$ electron beam polarization. The SLD is the modern multipurpose solenoidal detector at the SLC interaction region. It is designed to study the decays of the $\mathrm{Z}^{0}$ boson, and as such is well suited to identifying and measuring taus produced in $Z^{0}$ boson decay.

\subsection{The Polarized SLC}

The SLC is the only non-circular electron-positron collider in the world. It is located at the Stanford Linear Accelerator Center in Menlo Park, California. As a quasi-linear collider it has the unique ability to accelerate polarized electron beams without the depolarization due to spin precession in circular synchrotrons. Polarized electrons are provided by the polarized electron source. They are then accelerated by the 2 mile long linear accelerator and brought into collision by the beam arcs.[12]

\subsubsection{The Polarized Source}

At the SLC, electrons may be produced in two ways. A traditional thermionic gun is used to produce unpolarized electrons for machine tune up while the polarized source itself is being tuned up. This is essentially same as the electron gun at the back of a common cath-

1. SLAC Linear Collider

2. SLAC Large Detector 
ode ray tube and works by putting a voltage between a heated cathode and a suitable hole. For normal machine operations, a polarized gun is used. This uses a circularly polarized laser (currently a ND:YAG pumped Ti:Saphire laser) to selectively excite longitudinally polarized states in the conduction band of the Gallium-Arsenide (GaAs) crystal used as the cathode in the gun. The voltage across this cathode then strips these excited electrons and begins their acceleration.

Originally, for the 1992 run, a bulk GaAs crystal was used. This has a maximum theoretical polarization of 50\%. For the 1993 run, a "strained lattice" GaAs crystal was used. This is a thin layer of GaAs grown on a Gallium-Arsenide-Phosphide (GaAsP) crystal. The difference in lattice spacings between GaAs and GaAsP strains the lattice of the GaAs, shifting the excitation energies and allowing the maximum theoretical polarization to be $100 \%$. In 1993, this yielded a beam polarization of $63 \%$. For the $1994-5$ and 1996 runs, an improved, thinner version of this cathode yielded beam polarizations near $80 \%$.

Looking at Fig. 2.1, we see the energy levels in Bulk GaAs (top) and strained lattice GaAs (bottom). The solid lines represent transitions induced by right-hand circularly polarized light, and the dashed lines indicate those induced by left-handed light. The numbers in the circles indicate the relative sizes of the transition matrix elements. Thus a righthand circularly polarized laser tuned to excite $1.52 \mathrm{eV}$ transition will yield a theoretical maximum polarization of $50 \%$ for the bulk GaAs, since it will excite both the $m_{j}=-3 / 2$ to $m_{j}=-1 / 2$ and the $m_{j}=-1 / 2$ to $m_{j}=1 / 2$ in a 3 to 1 ratio. In the strained-lattice cathode, the degeneracy between these two transitions is broken and a properly tuned laser will only excite the $m_{j}=-3 / 2$ to $m_{j}=-1 / 2$ transition, yielding a theoretical maximum polarization of $100 \%$. Left-hand polarized light will excite the opposite transitions and result in oppositely polarized electrons.[13] 


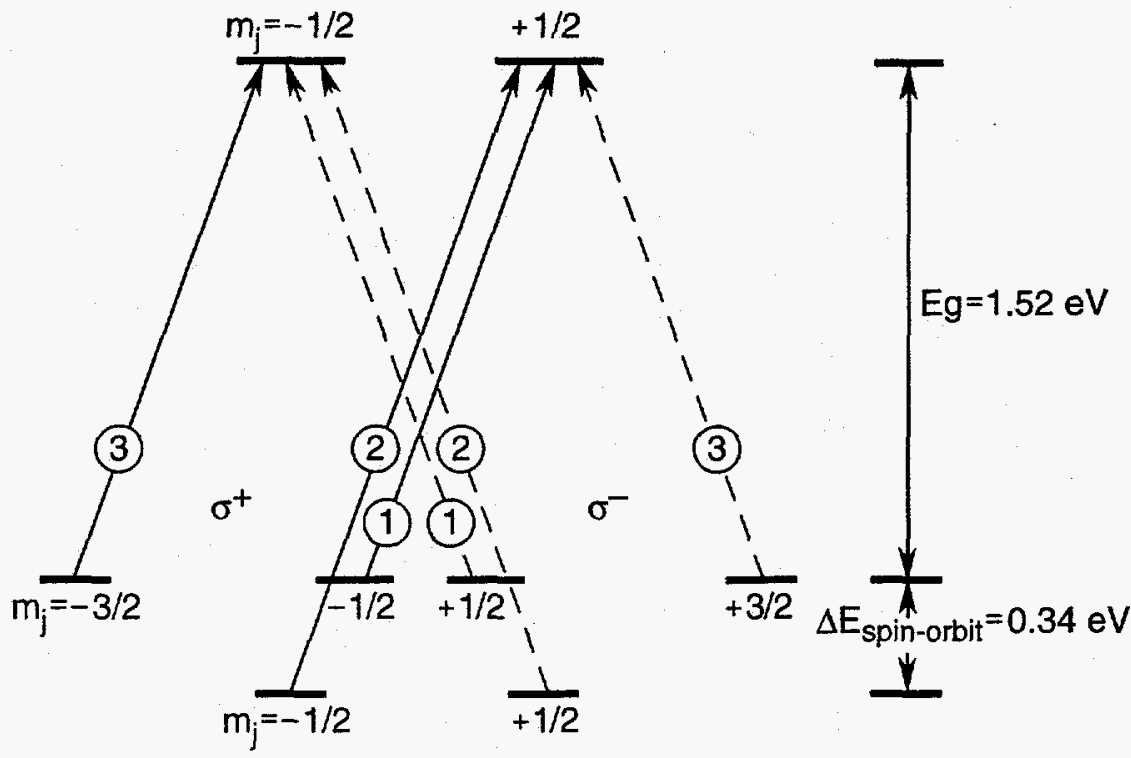

$\begin{aligned} & \text { Strained } \\ & \text { Induced } \\ & \text { Splitting }\end{aligned} \quad \| \begin{aligned} & \text { Strain Axis Parallel to } \\ & \text { Incident Photon Axis }\end{aligned}$

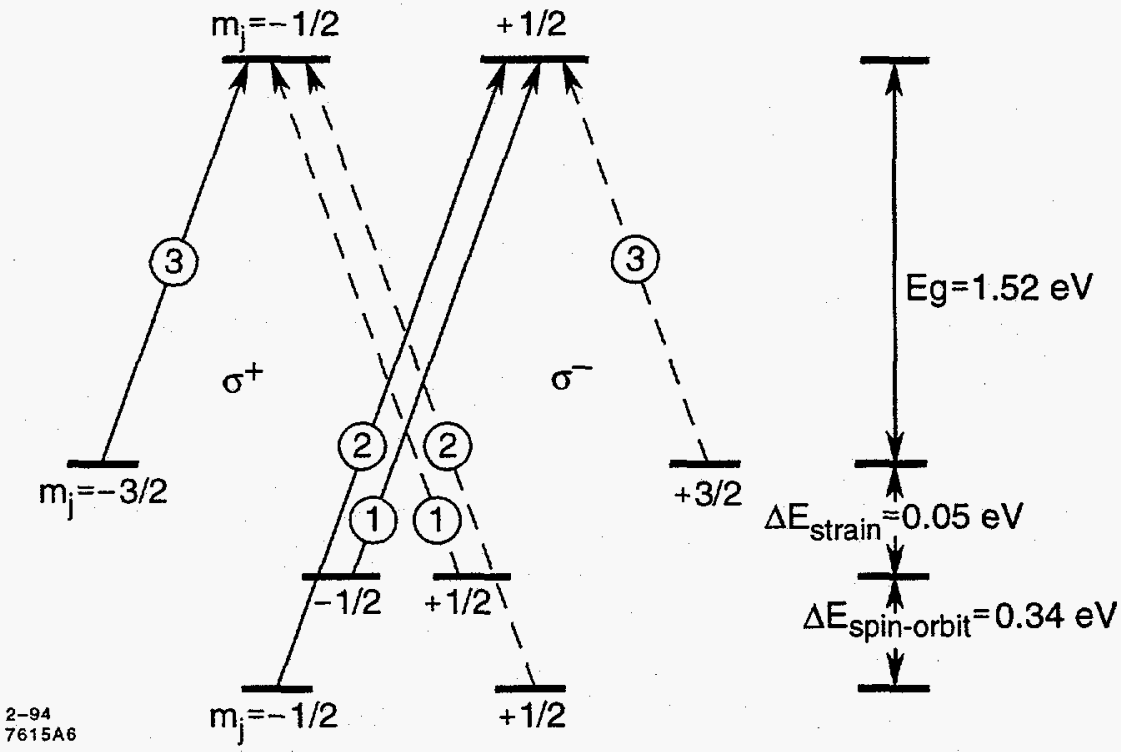

Figure 2.1: Energy levels in GaAs.

During normal polarized SLC operations, the machine operates at $120 \mathrm{~Hz}$. Usually, the sign of the polarization of the source laser and hence that of the electron beam polarization 
is varied randomly from pulse to pulse. Great care is taken to preserve this information and transmit it to the SLD data acquisition system and to the Compton Polarimeter.

\subsubsection{The Accelerator}

The SLC is based on the Stanford Linear Accelerator (linac), which was built in the 1960's to study the structure of nucleons by the scattering of $20 \mathrm{GeV}$ electron beams off of fixed targets. In the mid 1980's the linac was upgraded as a part of the new SLC designed to produce $\mathrm{Z}^{0}$ bosons at threshold (see Fig. 2.2).

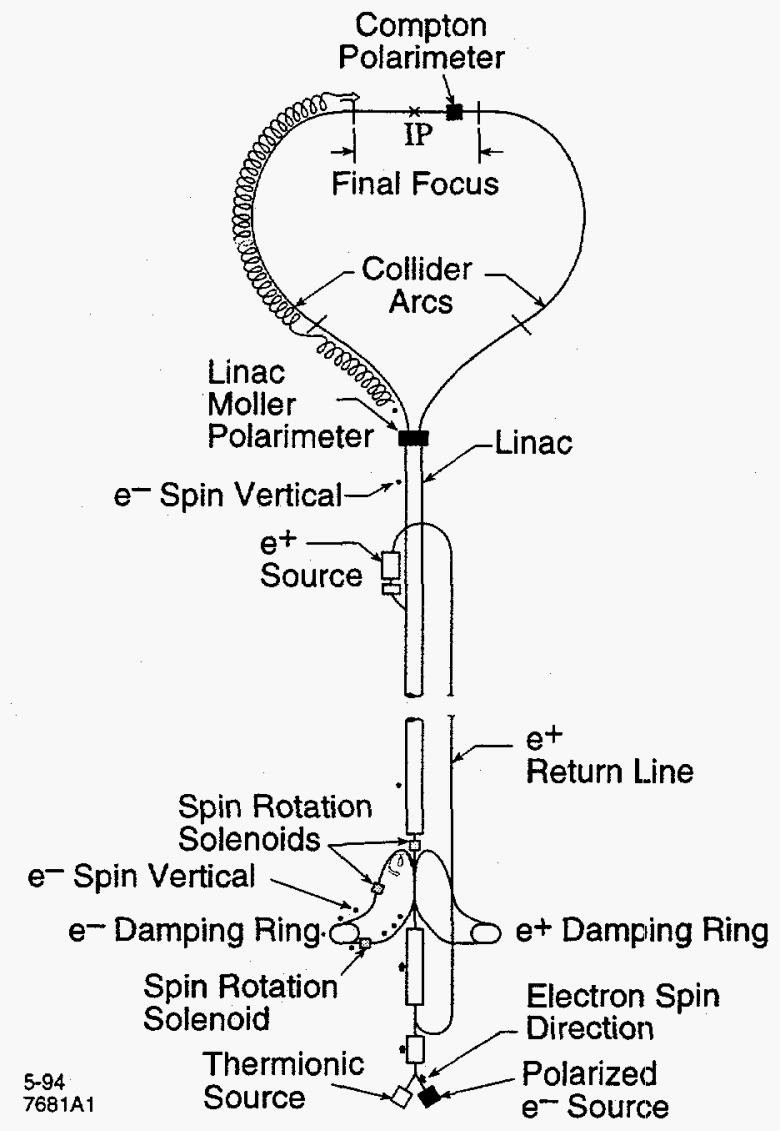

Figure 2.2: The SLC.

Under normal operating conditions, the SLC operates at $120 \mathrm{~Hz}$. In each cycle, the gun produces two bunches of longitudinally polarized electrons. These are accelerated down 
the linac to $1.19 \mathrm{GeV}$. They are then stored in the north damping ring for $8.3 \mathrm{~ms}$ which compresses the bunches and reduces their energy spread. Before entering the ring, the spins of the bunches are rotated by a solenoid so that they are transverse in the vertical direction. This prevents spin precession effects from depolarizing the beams in the damping ring. At the same time, a positron bunch is also accelerated to $1.19 \mathrm{GeV}$ and stored in the south damping ring. After their storage in the damping ring, the second electron bunch is accelerated to $30 \mathrm{GeV}$ and routed to a Tungsten-Rhenium alloy target, where it showers prodigiously. The positrons are separated out of this shower magnetically and routed to the beginning of the linac where, along with two new electron bunches, they participate in the next cycle of the SLC. The polarization of the electrons has no effect on the produced positrons. Meanwhile, the first electron bunch and the positron bunch from the previous cycle are extracted from the damping ring and accelerated in the linac to $46 \mathrm{GeV}$.

At the end of the Linac, these bunches are separated by a magnet and routed into the two mile-long beam arcs that bring the two beams into collision at the interaction point (IP) at the center of the SLD. Due to synchrotron radiation in the arcs, the beam energies are reduced from $46 \mathrm{GeV}$ to $45.63 \mathrm{GeV}$ (slightly more than half the $\mathrm{Z}^{0}$ boson mass) at the IP. The arcs have the potential to depolarize the electrons due to spin precession. As a result, the launch angle of the electron beam polarization into the North arc is carefully optimized to maximize the longitudinal electron beam polarization at the IP. After passing through the IP, the beams pass through the energy spectrometers, which measure their energy, and are dumped. Additionally, the electron beam is analyzed by the Compton polarimeter, which measures its longitudinal polarization.

Since it started running in 1987, the SLC has constantly been improved (Fig. 2.3 and Fig. 2.4). By the 1994-5 run, Luminosities of $0.7 \times 10^{30} \mathrm{~cm}^{2} \mathrm{~s}^{-1}$ had been achieved, and beam polarizations on the order of $80 \%$ were delivered to the IP. 
Beam Polarization

SLD 1992 - 1995 Data

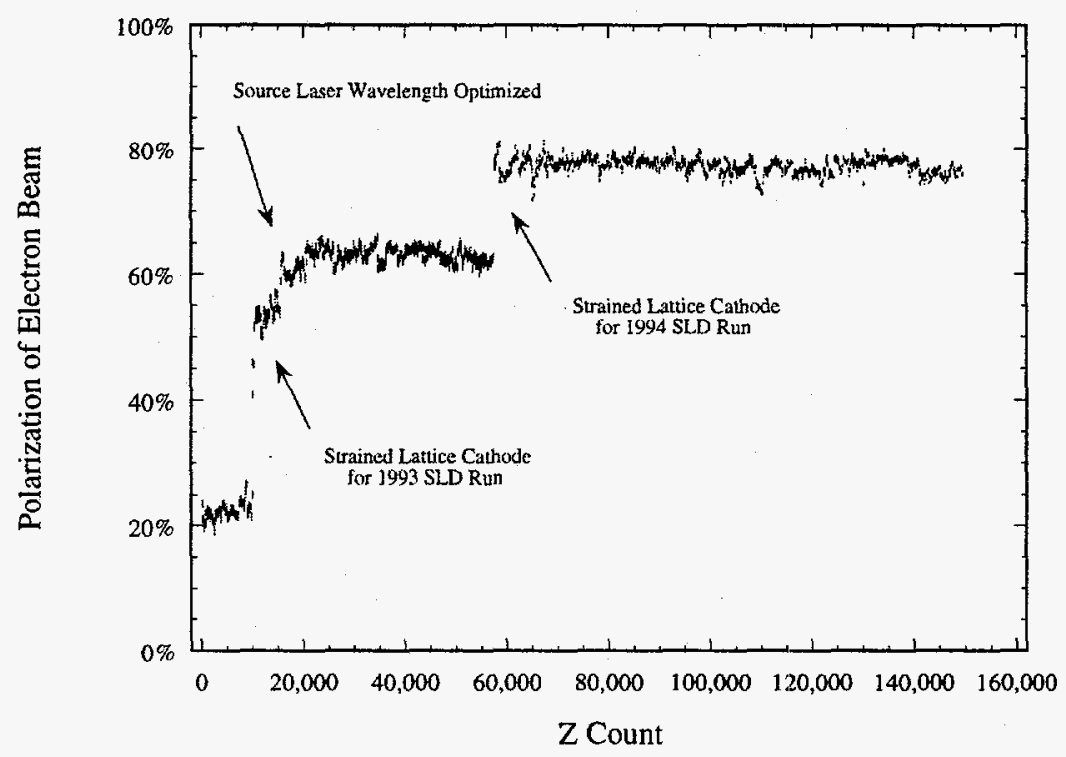

Figure 2.3: SLC polarization time history.

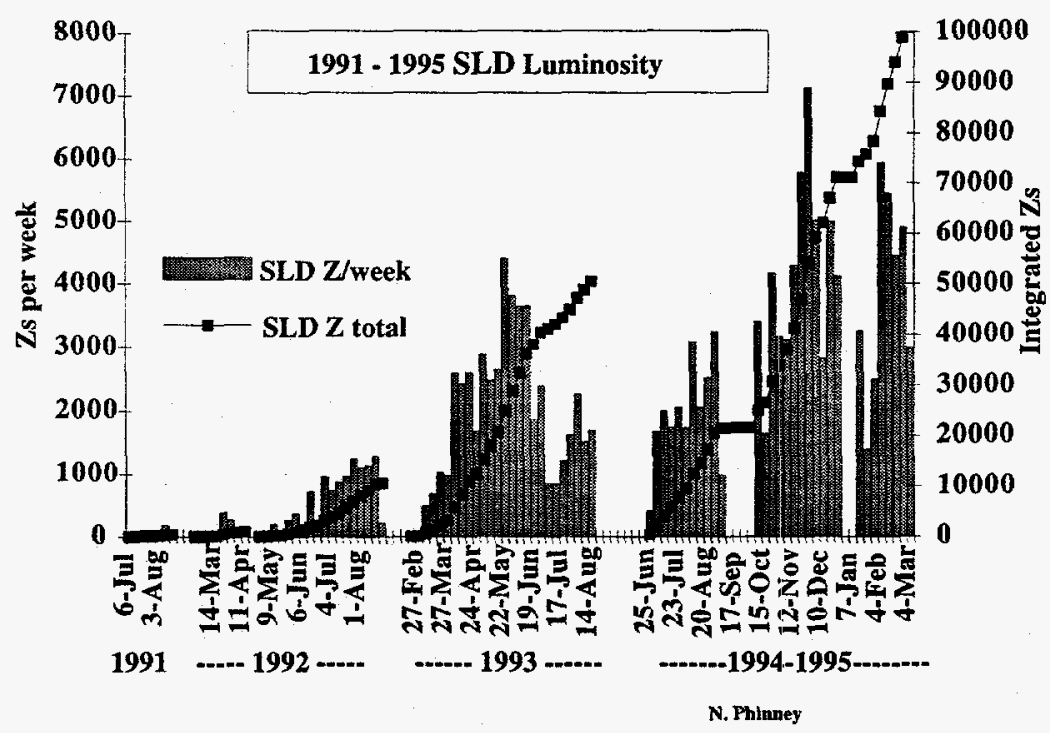

Figure 2.4: SLC luminosity time history. 
Table 2.1: SLC Performance 1993-95.

\begin{tabular}{l|l|l|l}
\hline \multicolumn{1}{c|}{ Run } & \multicolumn{1}{c|}{$\int \mathcal{L}$} & Hadronic $\mathrm{Z}^{0} \mathrm{~s}$ & \multicolumn{1}{c}{$<$ Pol $>$} \\
\hline 1993 & $1.78 \mathrm{pb}^{-1}$ & 49392 & $63.0 \pm 1.1 \%$ \\
\hline $1994-5$ & $3.66 \mathrm{pb}^{-1}$ & 92261 & $77.2 \pm 0.5 \%$ \\
\hline
\end{tabular}

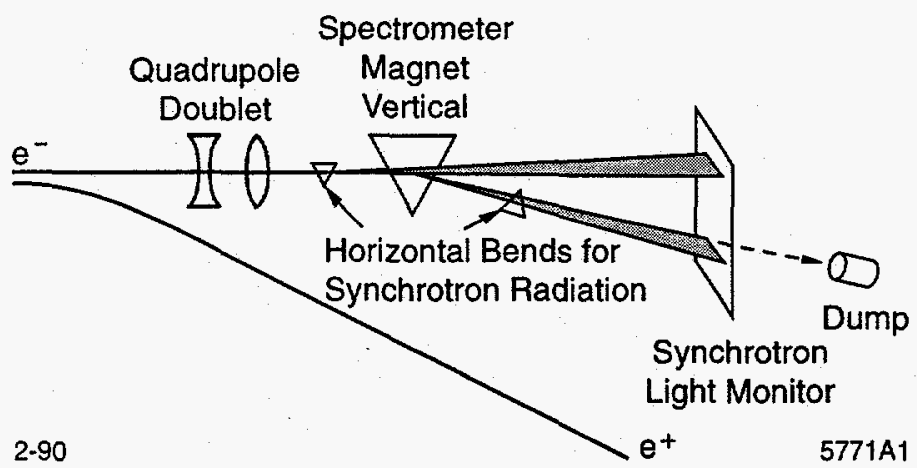

Figure 2.5: Energy spectrometer schematic.

\subsubsection{The Energy Spectrometers}

After passing through the SLD, both the electron and positron beams are separated from the incoming beam line and pass through the energy spectrometers before being dumped. These spectrometers consist of three dipole magnets. The first and last of these dipoles are designed to produce synchrotron light stripes from the beam and the middle dipole is a precision analysis magnet. The two synchrotron dipoles are oriented at right angles to the analysis magnet; as a result, the angle between the synchrotron stripes is the angle through which the analysis magnet has bent the beam. The synchrotron light stripes are measured by the Wire Imaging Synchrotron Radiation Detectors (WISRDs) which are located $15 \mathrm{~m}$ downstream from the analyzing magnet. These consist of two screens of 96 copper wires which measure the separation between the two synchrotron stripes. This separation is 
directly proportional to the beam deflection in the analysis magnet, which is inversely proportional to the beam energy. This results in a measurement of the single beam average energy to an error of $22 \mathrm{MeV}$.[14]

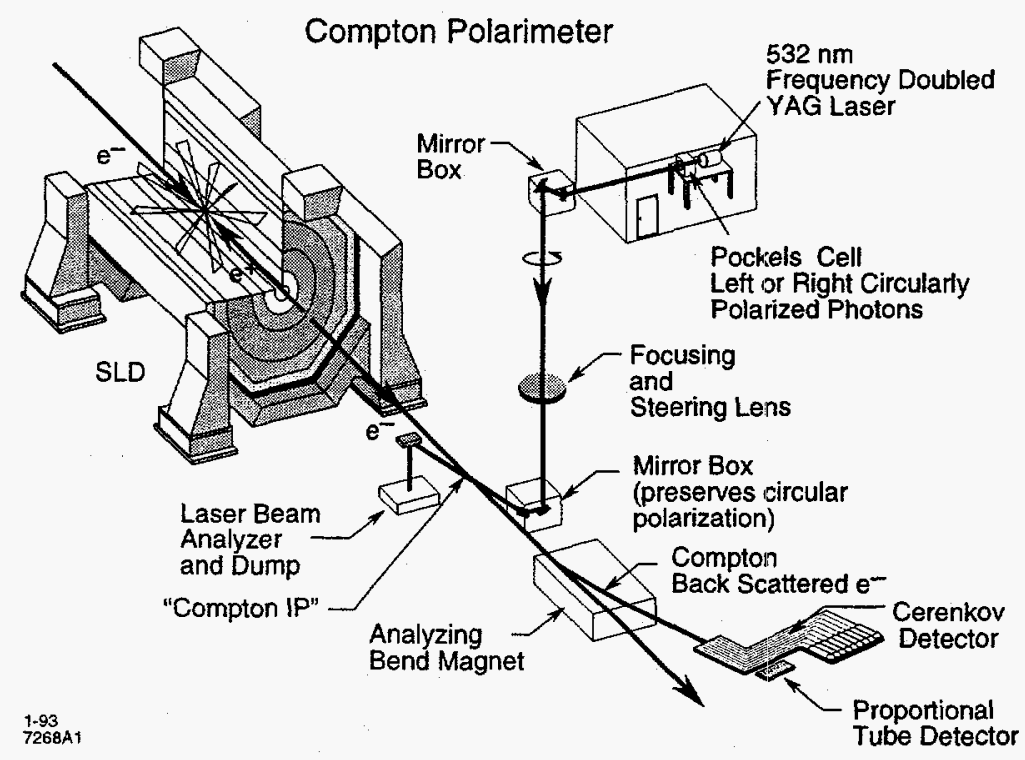

Figure 2.6: The Compton polarimeter.

\subsection{The Compton Polarimeter}

The Compton polarimeter measures the longitudinal polarization of the electron beam. It consists of a $532 \mathrm{~nm}$ frequency doubled Nd:YAG laser with some polarizing optics, an analyzing magnet and a threshold Cherenkov detector to detect electrons backscattered from the laser beam. The Compton polarimeter takes advantage of the cross section asymmetry in polarized Compton scattering of photons and electrons. Due to the high energy of the electrons $(45.63 \mathrm{GeV})$ compared to the photons $(2.33 \mathrm{eV})$, the Compton scattered electrons are highly collimated in the beam direction, with reduced energies down to a kinematic minimum at about $17 \mathrm{GeV}$. The analyzing magnet serves to separate these Compton scattered electrons from the beam and allow their energy spectrum to be measured. The 
laser light is brought into collision with the electron beam for every 11 th SLC beam pulse, for a rate of approximately $11 \mathrm{~Hz}$. The circular polarization of the light is varied randomly from pulse to pulse. We measure the resulting scattered electrons in the 7 channels of the Cherenkov detector which are arranged parallel to the beam line at varying distances corresponding to different bins in the scattered electron energy. Beam related backgrounds in the Cherenkov detector are determined from the laser-off beam pulses. From this, we can construct a cross section asymmetry for both left and right-handed beams between like and opposite polarized interactions for each channel of the Cherenkov detector. These can be compared with the well calculated polarized Compton cross section corrected for the EGS simulated detector response for each channel to give a polarization measurement.

During normal operations, a good polarization measurement can be obtained in about three minutes with a precision on the order of $1 \%$. In actuality, most of Compton polarimeter's time is spent doing various calibration scans to reduce the systematic errors on the polarization measurement. The final overall average magnitude of the beam polarization is $63.0 \pm 1.1 \%$ for the 1993 run and $77.2 \pm 0.5 \%$ for the $1994-5$ runs. [15]

\subsection{The SLD}

The SLD is solenoidal $4 \pi$ detector designed to analyze $Z^{0}$ boson decays at the SLC (Fig. 2.7 and Fig. 2.8). It is a $4.5 \mathrm{~m}$ radius cylinder of $10 \mathrm{~m}$ length centered on the SLC interaction point. It consists of a series of concentric subsystems designed to measure the energies and momenta of both charged and neutral particles resulting from $\mathrm{Z}^{0}$ decays as well as to determine their type. These subsystems generally consist of a cylindrical barrel system with a flat endcap system plugging either end. The SLD's basic structure consists of a $0.6 \mathrm{~T}$ solenoid and its requisite flux return. 


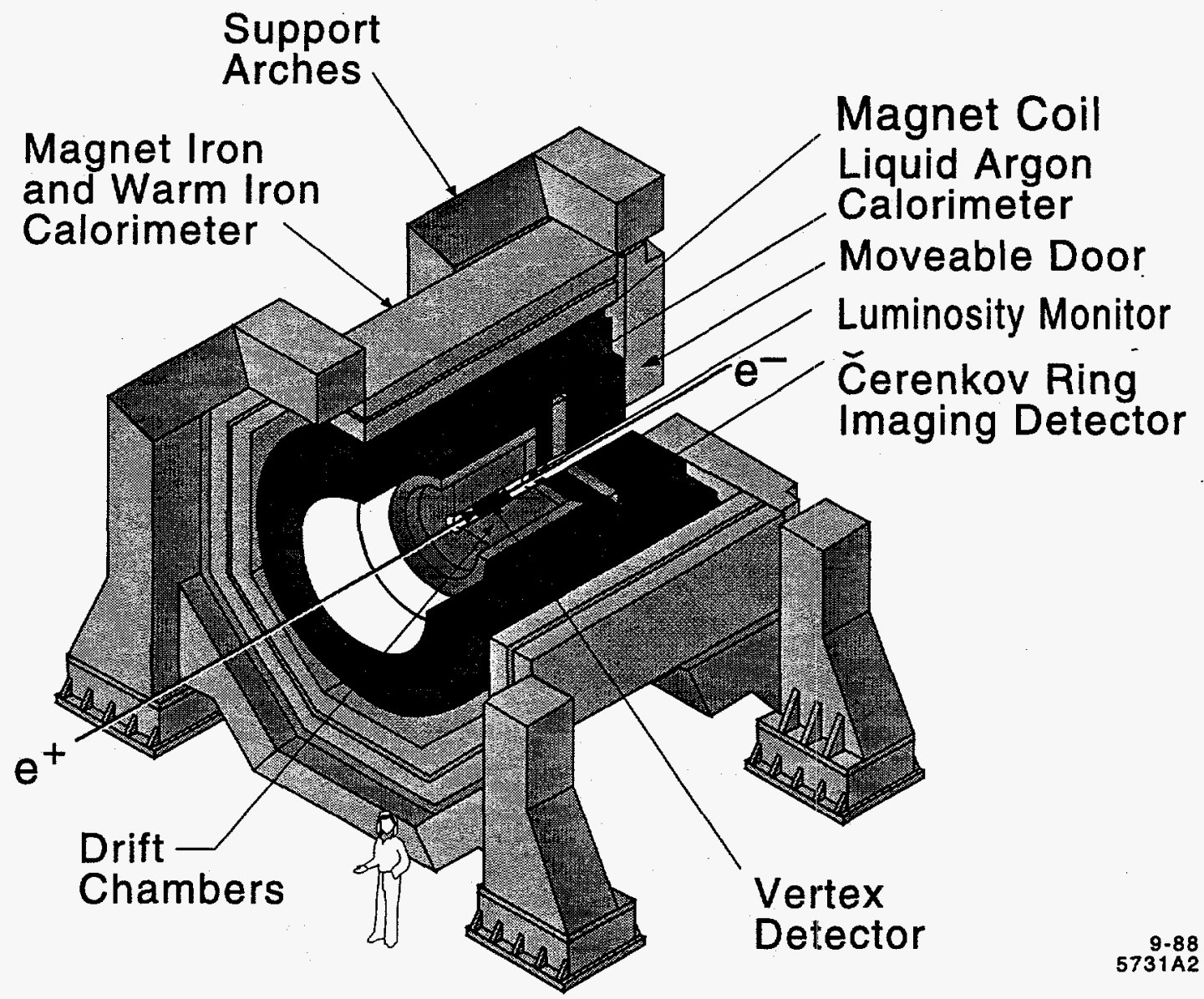

Figure 2.7: The SLD. (cut away view)

Starting from the IP, in the barrel we have the Vertex Detector (VXD) to determine precisely the starting points of charged tracks. Moving out, we have the Central Drift Chamber (CDC) to detect charged tracks and measure their momenta, the Cherenkov Ring Imaging Detector (CRID) to identify charged particles, and the Liquid Argon Calorimeter (LAC) to measure electromagnetic and hadronic shower energies of both charged and neutral particles. Outside this, we have the magnet coil and its requisite flux return, which is instrumented as the Warm Iron Calorimeter (WIC) and used primarily for muon identification. The endcap regions are slightly different in that there is no vertex detector, and there are endcap drift chambers both to the inside and to the outside of the endcap CRID. In 
addition, along the beampipe, there is a luminosity monitor (LUM) to measure the SLC luminosity through Bhabha scattering. The subsystems that are used in this analysis will be described in greater detail. In these descriptions, we will take the $\mathrm{z}$ axis as parallel to the beam line.[16]

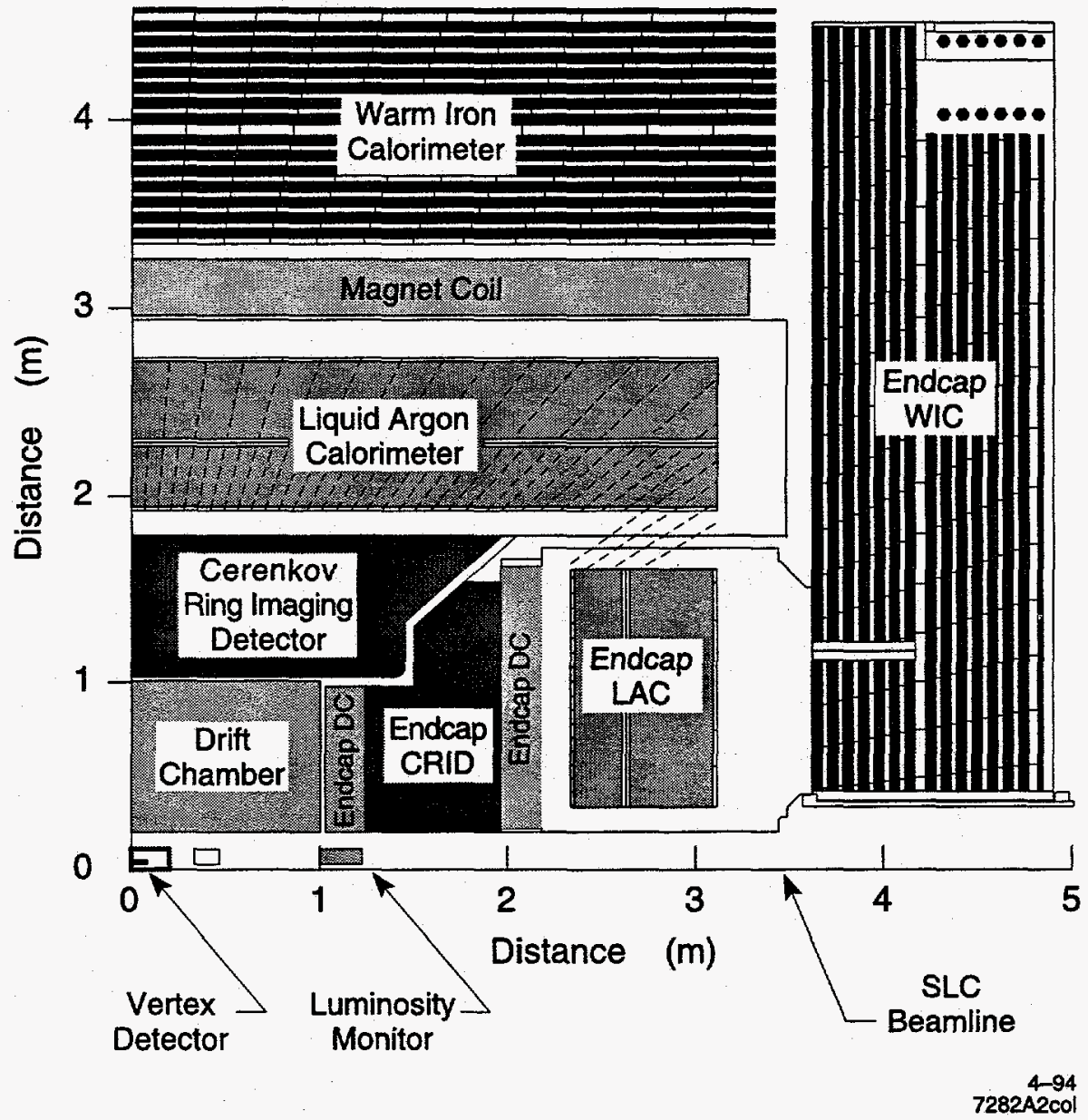

Figure 2.8: The SLD. (Quadrant view)

\subsubsection{The Vertex Detector}

The Vertex Detector is designed to provide a precision measurement of the initial trajectory of charged particles in the SLD. It consists of four concentric cylinders of CCDs arranged around the IP, with the inner barrel at radius $29.5 \mathrm{~mm}$, and the outer barrel at 
radius $41.5 \mathrm{~mm}$. These CCDs are arranged on $609.2 \mathrm{~cm}$ long ladders supported by a Beryllium shell. There are eight CCDs per ladder, and each $\mathrm{CCD}$ is about $1 \mathrm{~cm}$ square and consists of $\sim 200 \mathrm{k} 22 \times 22 \mathrm{~mm}$ square pixels. These are arranged so that a charged track traversing the detector within its fiducial volume $(\cos \theta<0.75)$ will hit an average of 2.3 pixels. The VXD and beampipe assembly contribute about $0.71 \%$ of a radiation length of material before the first layer of the CDC.

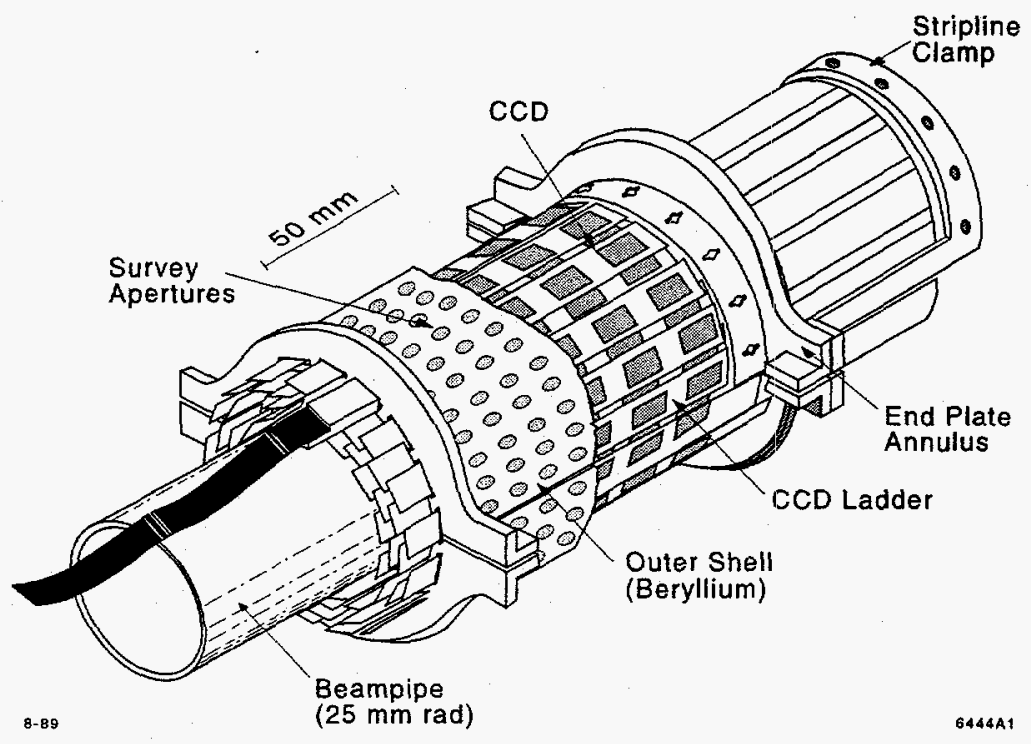

Figure 2.9: The SLD vertex detector. (VXD2)

The VXD resolution can be determined from tracks with three associated pixel hits. From this procedure, we find single hit resolutions of $\sim 5 \mu \mathrm{m}$ in the $\mathrm{r}-\phi$ plane (perpendicular

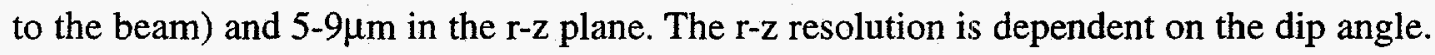
Utilizing the track information from the CDC, impact parameter resolutions of $\sim 12 \mu \mathrm{m}$ in the $\mathrm{r}-\phi$ plane and $38 \mu \mathrm{m}$ in the $\mathrm{r}-\mathrm{z}$ plane have been observed for $\mu$-pair events $(45.63 \mathrm{GeV}$ Tracks). For the 1996 run and beyond, the vertex detector has been replaced with an improved version with better position resolution and low angle coverage.[17] 


\subsubsection{The Central Drift Chamber}

The CDC tracks charged particles over $80 \%$ of $4 \pi$, utilizing track curvature due to the $0.6 \mathrm{~T}$ field of the SLD solenoid to measure their momenta. The chamber is $2 \mathrm{~m}$ long with a $1 \mathrm{~m}$ outer radius and a $20 \mathrm{~cm}$ inner radius. Charged particles are tracked using the charge that they deposit while traversing and ionizing the CDC gas. The deposited charge drifts to sense wires due to the electric field set up in the chamber by the field wires.

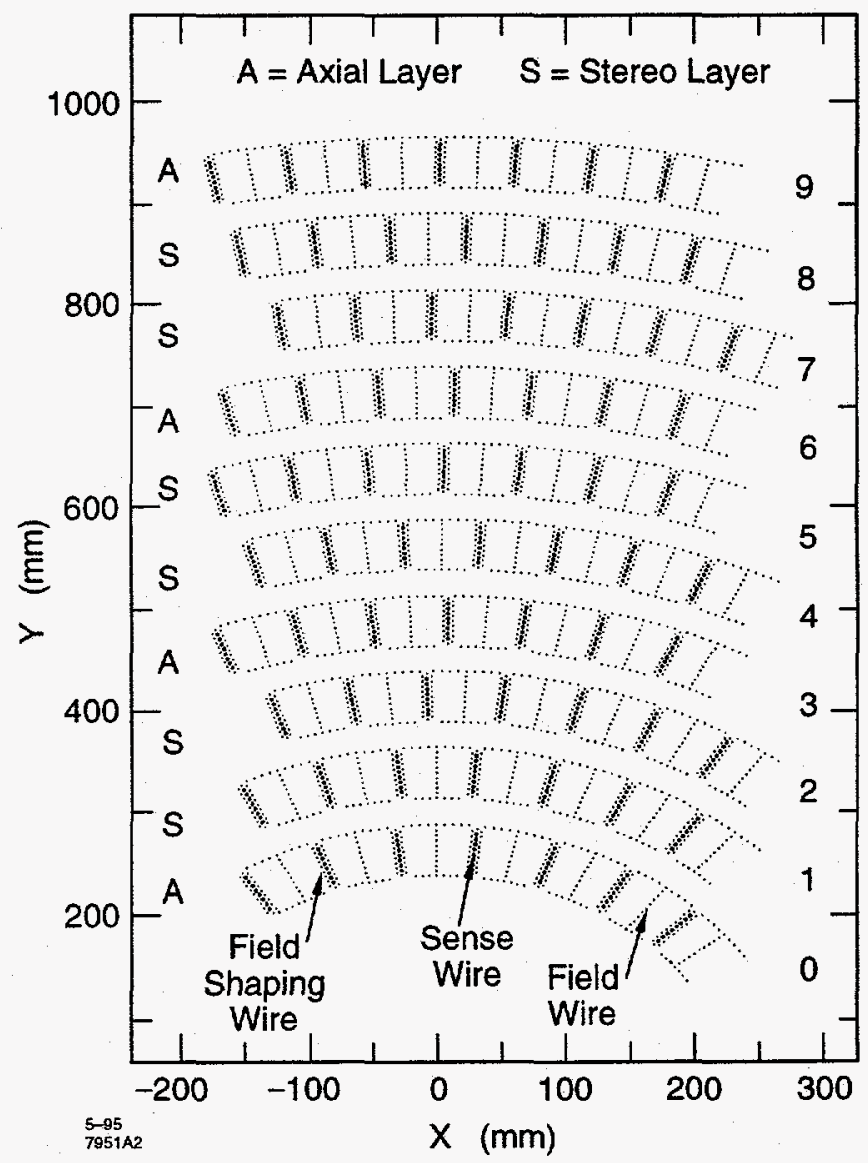

Figure 2.10: Partial CDC wire map.

There are 80 layers of sense wires arranged in 10 superlayers of eight wires each. Six of these superlayers have a $41 \mathrm{mrad}$ stereo angle with respect to the beam line alternating in the sign of the angle, while the remaining layers are parallel to the beam line (axial). 
The wires of each superlayer are arranged in cells roughly $6 \mathrm{~cm}$ wide by $5 \mathrm{~cm}$ high with the neighboring sense and field wires aligned along radii of the chamber. The electric field is generated by applying a voltage between the field wires and the field shaping and sense wires so that the deposited electrons drift to the sense wires. The field is uniform over most of the width of the cell. The gas is a mixture of $75 \% \mathrm{CO}_{2}, 21 \%$ argon, $4 \%$ isobutane, and $0.2 \%$ water, which has the advantage of a low drift velocity and diffusion constant. The drift velocity is approximately $7.9 \mu \mathrm{m} / \mathrm{ns}$ in the uniform field region, so the time from the beam crossing of a hit can be used to determine the drift distance.

Sense wire hits are read out from both ends so that charge division may be used to determine the $\mathrm{z}$ position to about $2 \mathrm{~cm}$. Multiple hits can be read from an individual wire with the drift time and known wire positions and electric field maps providing the hit locations in the $x-y$ plane. We find that the drift distance resolution is about $92 \mu \mathrm{m}$ in the flat field region away from the sense and field wires. Wire hits are grouped into vector hits, which consist of the best straight line fits to the individual wire hits within a given superlayer. These vector hits are then fed into a pattern recognition routine which groups them into tracks. The tracks are then fit for momentum and starting point, using the individual hit parameters and the known magnetic field. At this point, the charge division information is discarded as the stereo information is more accurate for $\mathrm{z}$ determination once hits have been grouped into tracks. This results in a momentum resolution of $\left(\sigma\left(\mathrm{p}_{\mathrm{t}}\right) / \mathrm{p}_{\mathrm{t}}{ }^{2}\right)^{2}=0.0050^{2}$ $+\left(0.010 / \mathrm{p}_{\mathrm{t}}\right)^{2}$, and an impact parameter resolution of $155 \mu \mathrm{m}$ in the r $\phi$ plane and $1.9 \mathrm{~mm}$ in the r-z plane. This can be improved by including VXD information for the tracks.[18]

\subsubsection{The Barrel Cherenkov Ring Imaging Detector}

The CRID is designed to identify the particle species of charged tracks. This is done by determining the velocity of a given track by measuring the Cherenkov angle of Cherenkov light emitted by the track. From this a mass can be determined and the species identified. 
For a particle of velocity $v=\beta c$ in a medium with index of refraction $n$, Cherenkov light is emitted if $\beta>1 / n$. This light is emitted at an angle, with $\cos \theta_{c}=1 /(\beta n)$, to the particle's direction of flight, so for a thin medium, this light will fall within a cone.

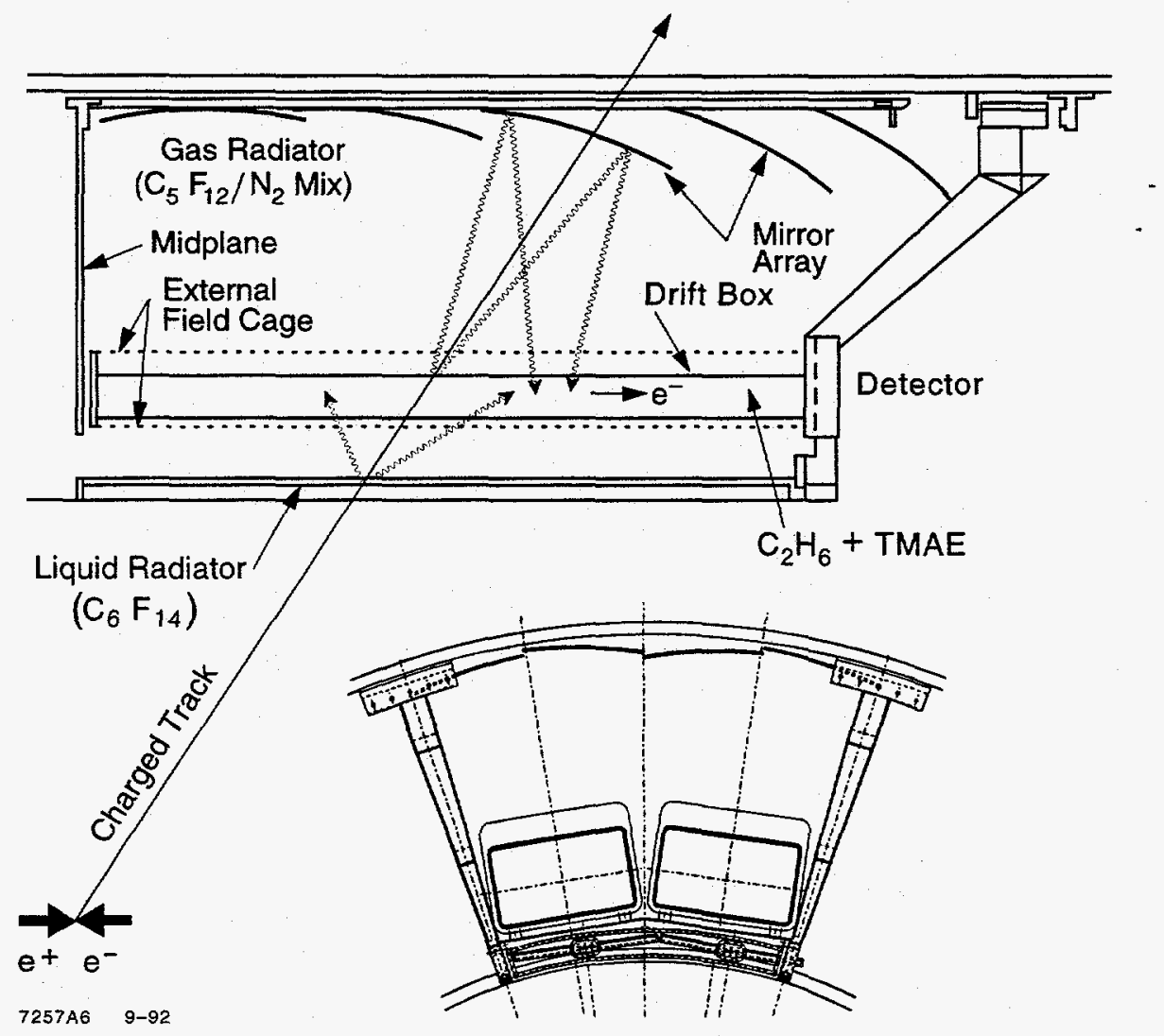

Figure 2.11: Barrel CRID detail.

The barrel CRID (Fig. 2.11) has two radiators, a thin liquid radiator of $\mathrm{C}_{6} \mathrm{~F}_{14}$ $(n=1.27802$ at $\lambda=1900 \AA)$ and a gas radiator of $C_{5} F_{12}(n=1.00163$ at $\lambda=1900 \AA)$. The light from the liquid radiator falls directly on the drift boxes of time projection chambers (TPCs), while the light from the gas radiator is focused on the TPCs by an array of spherical mirrors. In either case, we get a ring of Cherenkov light impinging on the TPC. The TPCs contain ethane and a small amount of the photo sensitive gas tetrakis(dimethylamino)ethylene (TMAE). The TMAE is ionized by the Cherenkov photons producing 
photoelectrons which drift to an array of wires at the end of the TPC. The charge is detected by the wires and the original ring can be reconstructed from the timing and location of the hits on the wires. The ring size may then be used in conjunction with the CDC momentum measurement to determine the particle's species. The liquid radiator's higher index of refraction allows it to cover low momenta while the gas covers higher momenta. $\pi / \mathrm{k} / \mathrm{p}$ separation is possible up to $30 \mathrm{GeV}$ and e/ $\pi$ separation up to $6 \mathrm{GeV}$.[19][20]

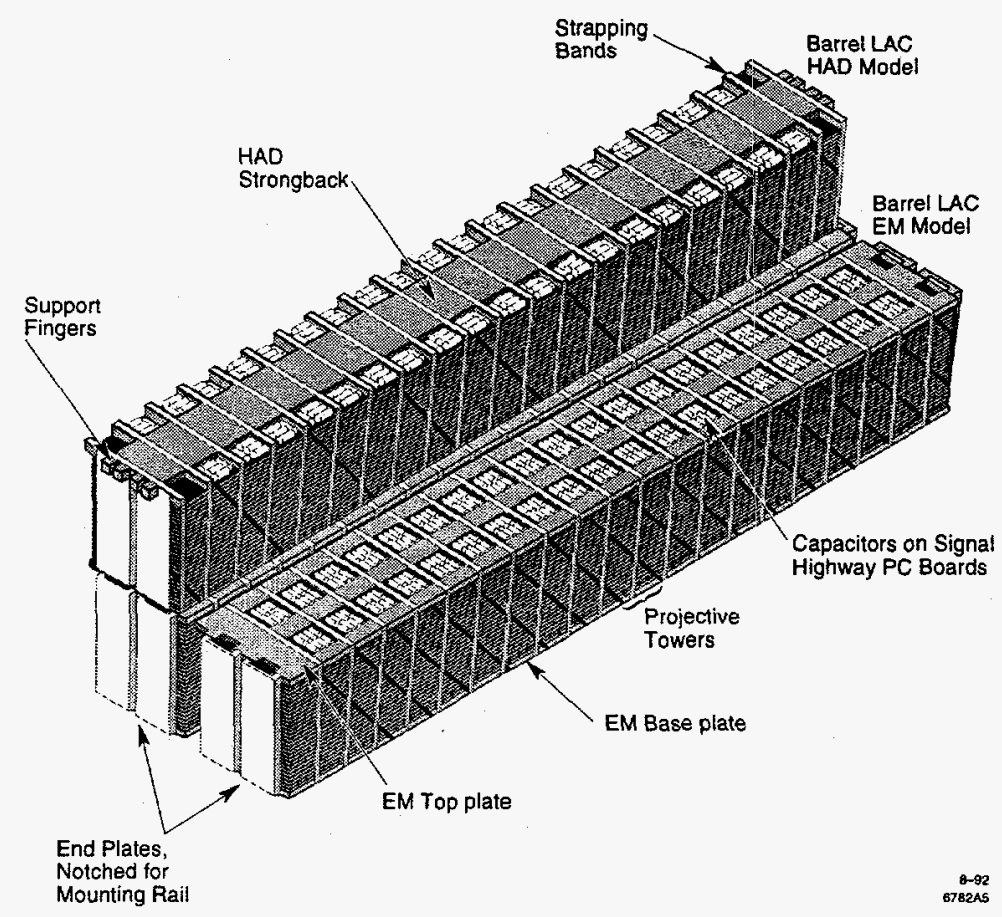

Figure 2.12: Typical barrel LAC modules.

\subsubsection{The Liquid Argon Calorimeter.}

The LAC is lead-liquid argon calorimeter designed to provide electromagnetic and hadronic calorimetry over $98 \%$ of $4 \pi$. It consists of a barrel section, covering polar angles greater than $33^{\circ}$ from the beam line $(|\cos \theta|<0.84)$, and two endcaps covering angles from $8^{\circ}$ to $35^{\circ}$ from the beam line $(0.82<|\cos \theta|<0.99)$, thus covering all $|\cos \theta|<0.99$ with an overlap region. The barrel is entirely within the magnet coil, so there are no energy losses 
due to interactions with the coil. The barrel is a hollow cylinder $6 \mathrm{~m}$ long with an inner radius of $1.8 \mathrm{~m}$ and an outer radius of $2.9 \mathrm{~m}$. The endcaps fit into either end of the barrel.

The LAC consists of lead plates alternating with lead tiles separated by spacers and bathed in 35,000 liters of liquid argon. The lead initiates showers and provides the bulk absorbing material, and the argon serves as the ionizing medium for shower sampling. The lead plates are grounded and the tiles are held at voltage to serve as a collection medium for the charge deposited in the argon. The LAC is segmented radially into an electromagnetic (EM) section and a hadronic (HAD) section. In the electromagnetic section, the plates and tiles are $2 \mathrm{~mm}$ thick with a $2.75 \mathrm{~mm}$ spacing for the argon. The plates in are arranged in a projective tower geometry in both the barrel and endcap. In the barrel (Fig. 2.12) there are 192 segments in azimuth each subtending $33 \mathrm{mrad}$ and 68 sections in polar angle varying from $21 \mathrm{mrad}$ to $36 \mathrm{mrad}$ near the endcaps. In the hadronic section, the tiles and plates are $6 \mathrm{~mm}$ thick with a $2.75 \mathrm{~mm}$ spacing for the argon. The tower geometry is continued in the hadronic sections with the plate sizes doubled in both azimuth and polar angle.

The electromagnetic section provides 21 radiation lengths of material or 0.8 nuclear absorption lengths. This contains all but $1-2 \%$ of the energy of a $50 \mathrm{GeV}$ electron shower. For readout purposes, the electromagnetic section is divided into two sections: EM1 consisting of the first 8 layers or 6 radiation lengths and EM2 consisting of the remaining 20 layers or 15 radiation lengths. The hadronic section provides an additional 2 nuclear absorption lengths for a total of 2.8 , which typically contain $80-90 \%$ of a hadronic shower. The hadronic section is also divided into 2 sections for readout purposes, HAD1 and HAD2, each of 13 layers or one nuclear absorption length. The EM energy resolution is $\sim 15 \% / \sqrt{\mathrm{E}}$, and the HAD energy resolution is $\sim 60 \% / \sqrt{\mathrm{E}}$, where $\mathrm{E}$ is in GeV.[21] 


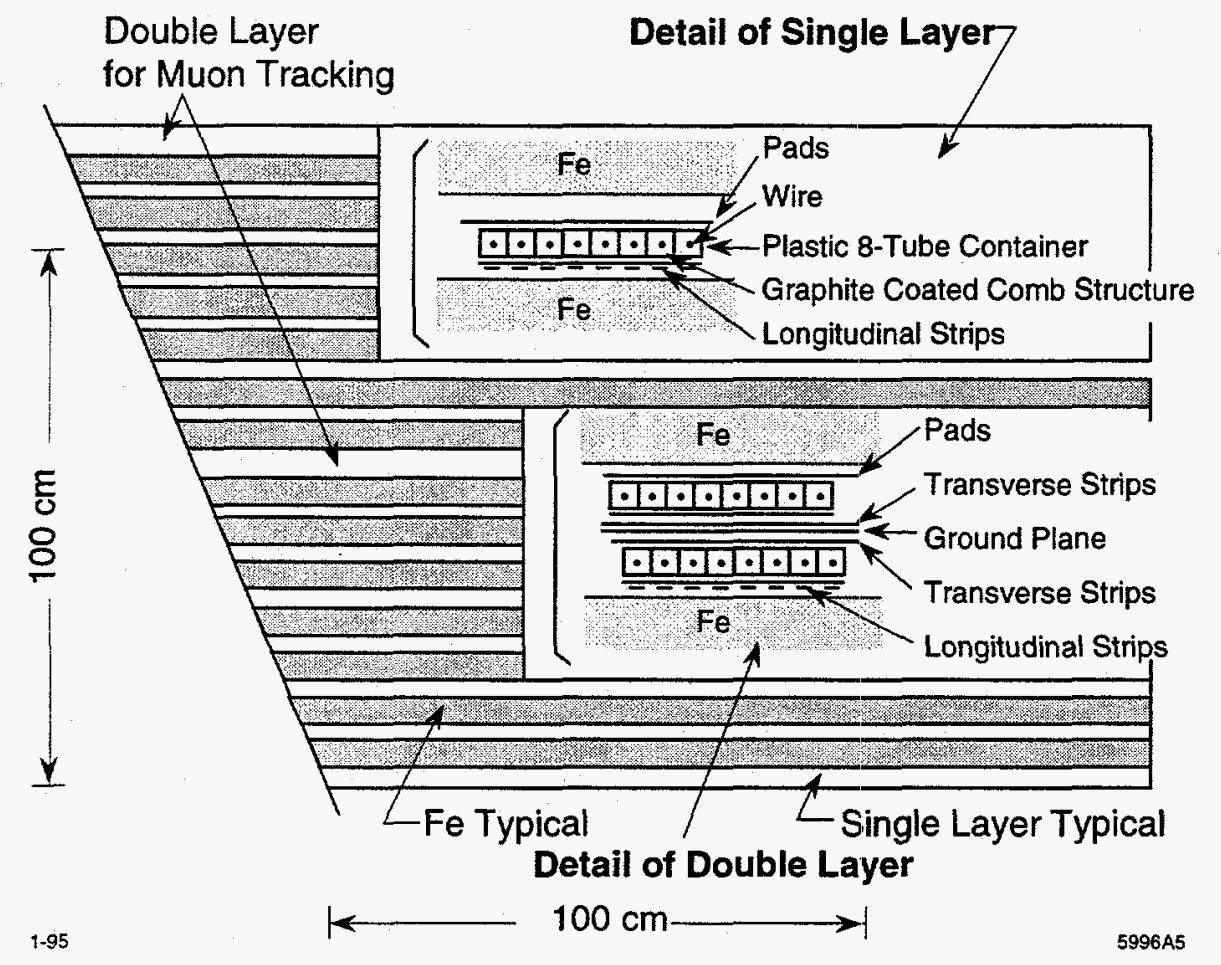

Figure 2.13: Barrel WIC details.

\subsubsection{The Warm Iron Calorimeter}

The WIC (Fig. 2.13) serves as the SLD solenoid's flux return and is designed to provide hadronic calorimetry beyond the LAC as well as identifying and tracking muons. It consists of 2 sets of seven $5 \mathrm{~cm}$ steel plates. These are instrumented with Iarocci limited streamer tubes. These are read out with pads which continue the projective tower geometry of the HAD sections of the LAC, and with both transverse and longitudinal strips for muon tracking and identification. Each set of Iarocci tubes is read out on both sides, typically one side has pads to provide tail catching calorimetry and the other has $1 \mathrm{~cm}$ wide longitudinal strips for muon tracking and identification. The first set of larocci tubes is read out with pads on both sides to measure showers produced in the magnet coil. The layers after the seventh and fourteenth steel plates are special double layers with one set of 
tubes having $4 \mathrm{~cm}$ wide transverse strips and the usual pads, and the other having the usual $1 \mathrm{~cm}$ longitudinal strips and another set of $4 \mathrm{~cm}$ transverse strips.

The WIC is primarily used for muon identification. We expect that most muons with momenta above $2.5 \mathrm{GeV}$ will completely penetrate the LAC and WIC. The LAC is 2.8 nuclear interaction lengths thick, the magnet coil adds another 0.6 , and the WIC itself is 4 interaction lengths. Thus, it is extremely unlikely that other particles will completely penetrate the WIC. As a result, it can be assumed that those that do so are muons. [22][23] 


\section{Chapter 3}

\section{Triggering and Reconstruction}

In order to be considered for this analysis, events must meet a certain criteria. First of all, they must be recorded by the SLD data acquisition system. The SLD data acquisition records only those events that satisfy one of several triggers based on some basic event quantities. Then, they must be reconstructed by the SLD off-line data processing system. The off-line reconstruction is only done for those events that pass a more stringent set of criteria called the Pass 1 filter.

\subsection{Triggers}

The SLC collides at a rate of $120 \mathrm{~Hz}$. The SLD data acquisition is capable of recording events at several Hertz. As a result, we must be somewhat selective in which events we record. Luckily, at the SLC, deciding which events to record to tape (triggering) is relatively easy. Event rates are low, and the interesting physics (multihadronic $\mathrm{Z}^{0}$ events, tau pairs, mu pairs, and wide angle Bhabha events) is quite distinct from the background (beam gas, accelerator related noise, and detector noise). As a result, the SLD acquisition triggers are fairly simple and intuitive. During the 1993 and 1994-5 runs, four triggers were significant for recording tau pair events: the track trigger, the hadron trigger, the energy trigger, and the wide angle Bhabha (WAB) trigger. During typical quiet running the overall trigger rate is on the order of $0.25 \mathrm{~Hz}$, although it can reach several Hertz during noisy conditions. 


\subsubsection{The Track Trigger}

The track trigger is based on CDC hits. Tracks are created out of patterns of hit cells. A cell is considered hit if six of its eight wires have hits on them. The hit cells in the event are then compared to a lookup table of hit patterns that correspond to all possible tracks with $\mathrm{p}_{\mathrm{t}}>250 \mathrm{MeV}$ originating from the IP. If a pattern of hit cells in an event corresponds to a pattern in the lookup table for at least nine out of the ten superlayers in the CDC, that pattern is called a track. The trigger is satisfied if there are two tracks at least $120^{\circ}$ apart in azimuth in the event. To avoid background this trigger is vetoed and the event is not recorded if more than 275 of the 640 CDC cells are hit. During the 1993 run, this veto threshold was adjusted several times, but this had little effect on triggering for tau events.

\subsubsection{The Energy Trigger}

The energy trigger is based on LAC information. LAC hits are classified using two thresholds, the low threshold, which is just below the amount of energy a minimum ionizing particle (MIP) would deposit in the LAC, and the high threshold, which is just above the amount of energy a MIP would deposit in the LAC. The energy trigger is satisfied if there are at least eight high threshold LAC hits in the event and the sum of the energy, using the minimum ionizing scale, in these hits is greater than $6 \mathrm{GeV}$. The trigger is vetoed if there are more than 1000 low threshold hits in the LAC. Until late 1994, only the calorimeter subsystems of the SLD (LAC, WIC, \& LUM) were read out for this trigger. As a result, since our tau analyses require tracking, this trigger was not important for taus until then.

\subsubsection{The Hadron Trigger}

The hadron trigger uses both CDC and LAC information, and is a hybrid between the energy and track triggers. The trigger is satisfied if there is at least one track defined as in the track trigger and the total high threshold LAC energy in the event is greater than 2 
$\mathrm{GeV}$ for the event. This trigger is vetoed if more than $275 \mathrm{CDC}$ cells have hits.

\subsubsection{The WAB Trigger}

The WAB trigger is based on LAC information from the electromagnetic sections only. The trigger is satisfied if the total energy in the EM LAC in high threshold hits is greater than $15 \mathrm{GeV}$ using the minimum ionizing scale. The trigger is vetoed if there are more than 1000 low threshold hits in the event.

\subsubsection{Other Triggers}

In addition to the above triggers, several other triggers were used during the 1993 and 1994-5 SLD runs. These had no impact on triggering for tau pair events, but we mention them here for completeness. The luminosity Bhabha trigger is designed to record low angle Bhabha events in the luminosity monitor. The muon trigger was designed to pick up mu pair events, but was extremely inefficient and was not used in 1994-5. The random trigger records an event every $20 \mathrm{sec}$. regardless of the detector state and is useful for studying backgrounds. [24]

\subsection{Trigger Efficiencies for Tau Pair Events}

For many analyses, it is useful to know how efficient the data acquisition system is for triggering on tau pair events. This can be studied in several ways. The two most straightforward ways are Monte Carlo simulation of tau pair events and their interaction with the detector, and studying the correlations between the various triggers in the real data.

\subsubsection{Monte Carlo Simulation of trigger efficiency}

We can estimate the trigger efficiency by looking at the fraction of simulated tau pairs that pass a simulation of the trigger. To simulate the SLD trigger, we use the KORALZ Monte Carlo[25] to generate $\mathrm{e}^{+} \mathrm{e}^{-} \rightarrow \mathrm{Z}^{0} \rightarrow \tau^{+} \tau^{-}$events and the resulting tau decays. We 
then run these through a GEANT[26] based simulation of the SLD detector after adding in random trigger data to reproduce machine related backgrounds. The detector simulation generates data identical in form to the data written from the various SLD subsystems for actual triggers. Unfortunately this has some limitations for trigger simulation, as this simulation is optimized to reproduce the actual data as it is written to tape, which differs somewhat from the on-line data available to the triggering system. This is particularly true for the $C D C$, where the trigger simulation does not handle charge division well and loses more hits in the central region of the barrel than actually occurs. For the calorimeter based triggers, the on-line data available to the trigger is virtually identical to the data that is written to tape, so we expect their simulation to be good.

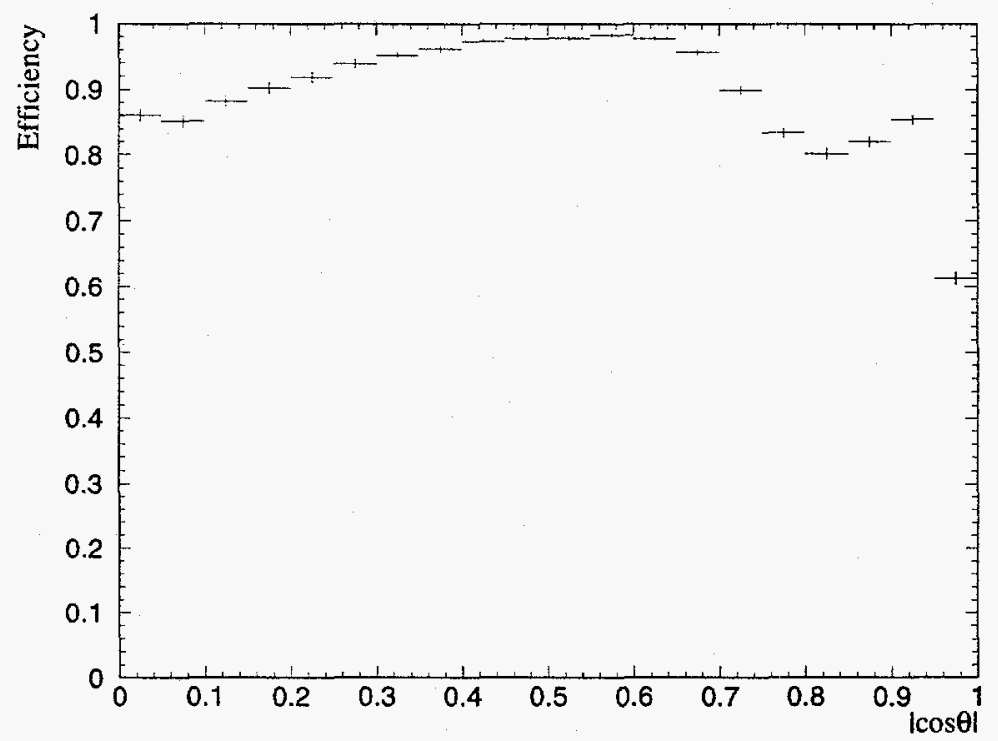

Figure 3.1: Overall trigger efficiency from tau Monte Carlo events.

Monte Carlo simulation of the trigger results in an efficiency curve (Fig. 3.1) with respect to the polar angle of the tau production direction. Due to the symmetry of the detector, we expect efficiencies to be flat in azimuthal angle and variable in the polar 
angle, which turns out to be the case. We can further break the efficiency down by individual trigger (Fig. 3.2).

Looking at the individual trigger breakdown, it is clear that the tracking based triggers may be having problems in the central region of the barrel, but we expect there to be some limitations of the simulation in this area. The data indicate that the combined trigger is nearly $100 \%$ efficient for $|\cos \theta|<0.7$ (see section 3.2 .3 ). Thus, it seems that the tracking trigger simulation is suspect in the central region of the barrel. The simulation seems to do a reasonable job at lower angles where it correctly models the drop in the tracking triggers due to the nine layer requirement to define a track. At $|\cos \theta|>0.71$, it is impossible for a track to intersect nine layers.
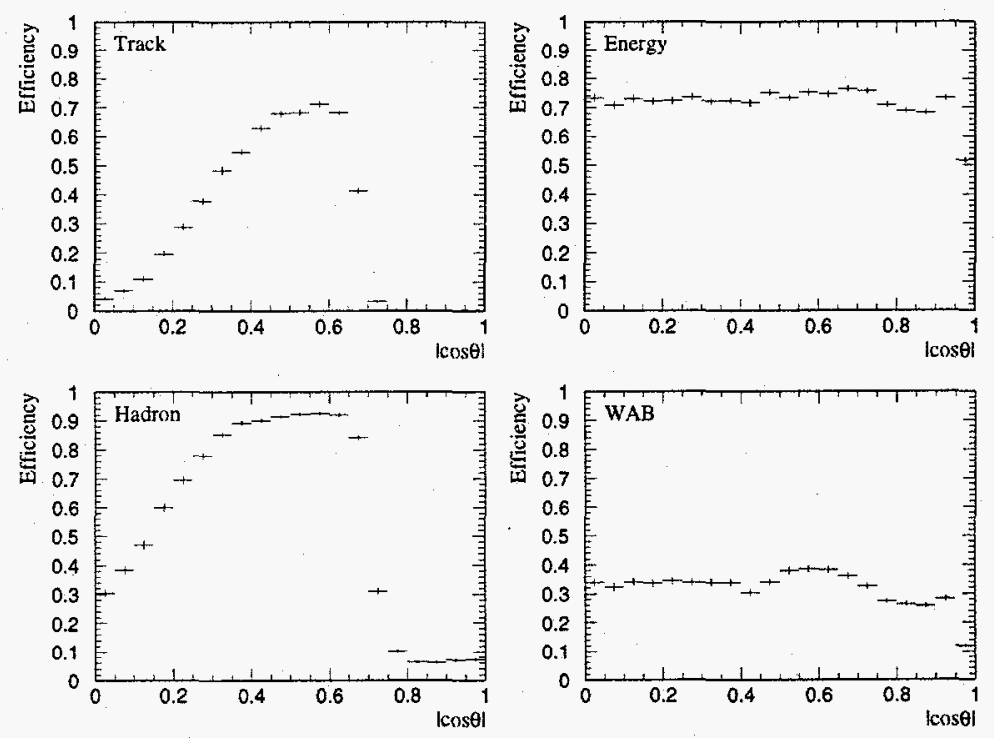

Figure 3.2: Individual trigger efficiencies from tau Monte Carlo events.

\subsubsection{Endcap Trigger Efficiencies}

In the endcap regions the only effective triggers are the calorimetry based energy and WAB triggers. Since the WAB trigger is essentially a subset of the energy trigger we con- 
fine the study to the energy trigger. In this region the primary factor affecting triggering for an event is the tau decays involved in the event. To study this we have broken tau decays into six basic categories:

- e $-\tau \rightarrow \mathrm{ev} \nabla$

$\cdot \mu-\tau \rightarrow \mu \nu \nabla$

$\cdot \pi-\tau \rightarrow \pi v$

- $\pi \pi^{0}$ - any decay with a charged pion and one or more neutral pions, including rho mesons and $\mathrm{a}_{1} \mathrm{~s}$ as well as nonresonant decays.

- $3 \pi$ - any decay involving three charged pions including $a_{1} s$ and nonresonant decays.

- other - all other decays.

For the endcap region, we get an overall energy trigger efficiency of $69 \%$ and efficiencies for various decay combinations according to Table 3.1. This looks pretty much as we expect with the electronic and multipion decays having high trigger rates due to the large amount of energy they deposit in the calorimeter and the muons having low trigger rates due to their low minimum ionizing energy depositions.

Table 3.1: Endcap Energy Trigger Rates for Various Decay Combinations.

\begin{tabular}{l|l|l|l|l|l|l|l}
\hline & $\mathrm{e}$ & $\mu$ & $\pi$ & $\pi \pi^{0}$ & $3 \pi$ & other & overall \\
\hline $\mathrm{e}$ & 0.70 & 0.30 & 0.61 & 0.84 & 0.80 & 0.66 & 0.68 \\
\hline$\mu$ & & 0.02 & 0.20 & 0.62 & 0.54 & 0.41 & 0.39 \\
\hline$\pi$ & & & 0.45 & 0.80 & 0.77 & 0.56 & 0.60 \\
\hline$\pi \pi^{0}$ & & & & 0.91 & 0.89 & 0.87 & 0.82 \\
\hline $3 \pi$ & & & & & 0.91 & 0.83 & 0.80 \\
\hline other & & & & & & 0.63 & 0.70 \\
\hline total & & & & & & & 0.69 \\
\hline
\end{tabular}

\subsubsection{Trigger Efficiencies from Correlations in the Data}

Since we have several triggers, we can look at correlations between triggers to determine 
their efficiencies. First we select a sample of tau pair events from the data (see Chapter 4). From these events, we then select the events that satisfy one trigger (B) and look at the fraction of those that satisfy a second trigger (A). If the two triggers are not correlated with each other or the tau filter, we expect this to give a reasonable estimate of the efficiency of the second trigger.

$$
\varepsilon_{A} \sim \frac{N_{A B}}{N_{B}}
$$

The tau filter is looser than the triggers in its tracking and energy requirements, so we expect it to have little correlation with the triggers. We should be able to use the events that satisfy the WAB and energy triggers to measure the track trigger efficiency and vice versa as the first two are wholly calorimeter based and the track trigger is entirely CDC based (see Fig. 3.5, Fig. 3.3 and Fig. 3.4). In the case of the energy trigger, we must be careful to use only those events after the full detector read out was installed. In all cases, we use the 1994-5 run data only.

From these measurements, it is apparent that the Monte Carlo does a good job modeling the energy and WAB triggers, and a not so good job of modeling the track trigger. The results we get for the track trigger efficiency differ significantly between the correlations with the energy and WAB triggers. We expect that the WAB trigger will pick up events with tau decays that have a lot of electromagnetic energy, the electronic decays and those involving neutral pions. Electrons have a lower tracking efficiency than most other charged particles due to their higher rates electromagnetic interactions with material before the CDC in the beam pipe or VXD. A decay with a particularly energetic neutral pion(s) will have the highest chance of firing the WAB trigger. It will also have a relatively low energy charged pion, which is least likely to be recognized as a track. As a result, we expect WAB triggered events to give a lower estimate of the tracking trigger efficiency. 


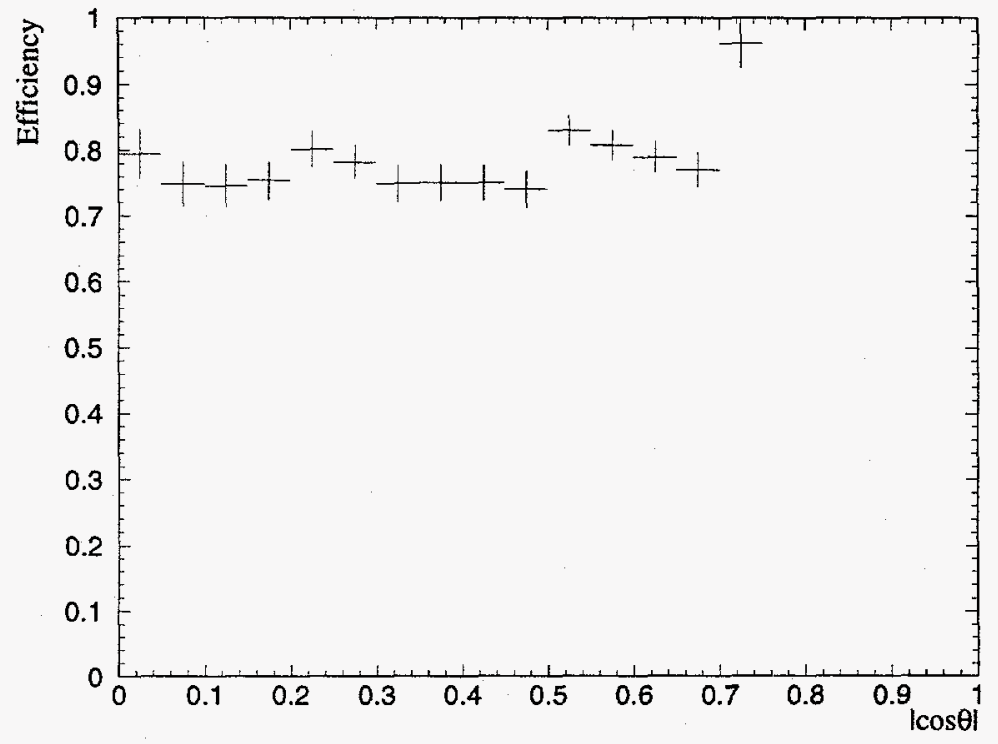

Figure 3.3: Energy trigger efficiency estimate from correlations.

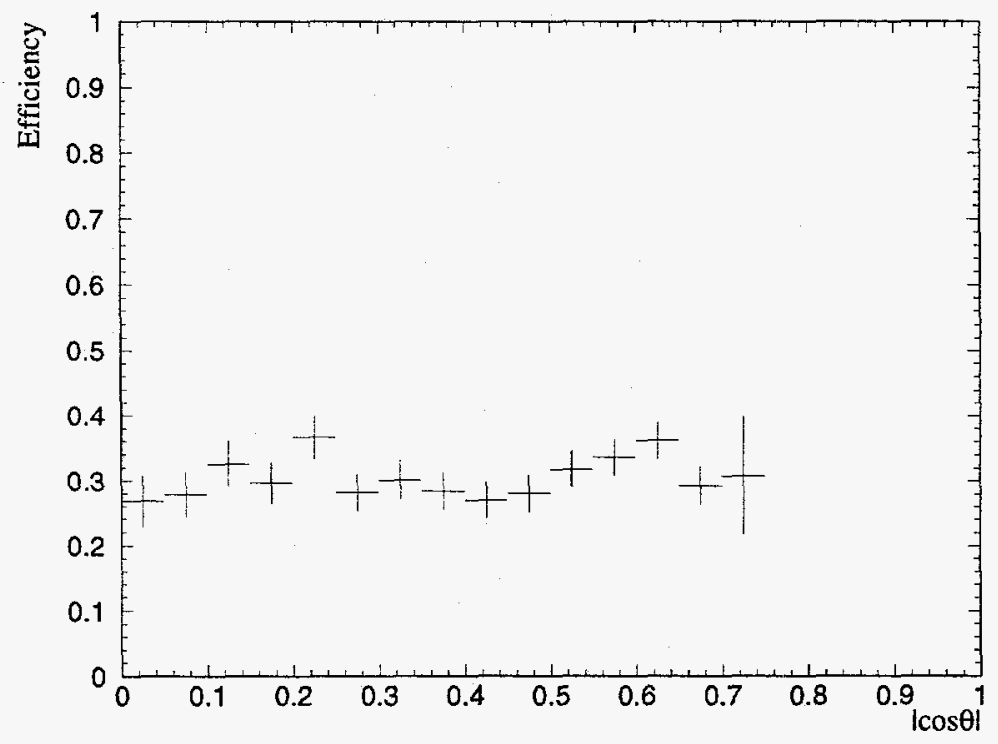

Figure 3.4: WAB trigger efficiency estimate from correlations. 


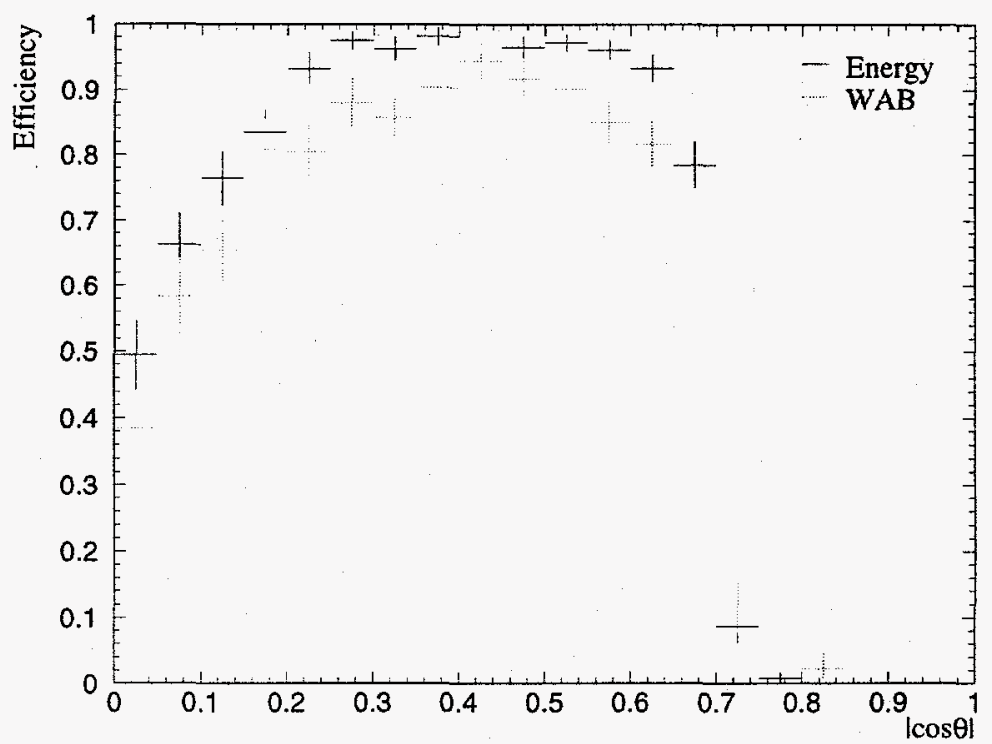

Figure 3.5: Track trigger efficiency estimates from correlations.

The Hadron trigger efficiency is more difficult to estimate as it combines calorimetric and tracking information, and thus is correlated with all of the other triggers. The correlations with the track trigger should tell us the fraction of events that fail to make the hadron trigger's energy cutoff, and the correlations with the calorimetric triggers should tell us the fraction events that fail to have a track. The overall hadron trigger efficiency should be something like the product of these two efficiencies. (See Fig. 3.6.) The combined efficiency curve is pretty much what we expect. There should be a drop off in the center of the detector due to charge division degrading the track finding, but since only one track is required for the hadron trigger, the hole should be smaller than that for the track trigger. 


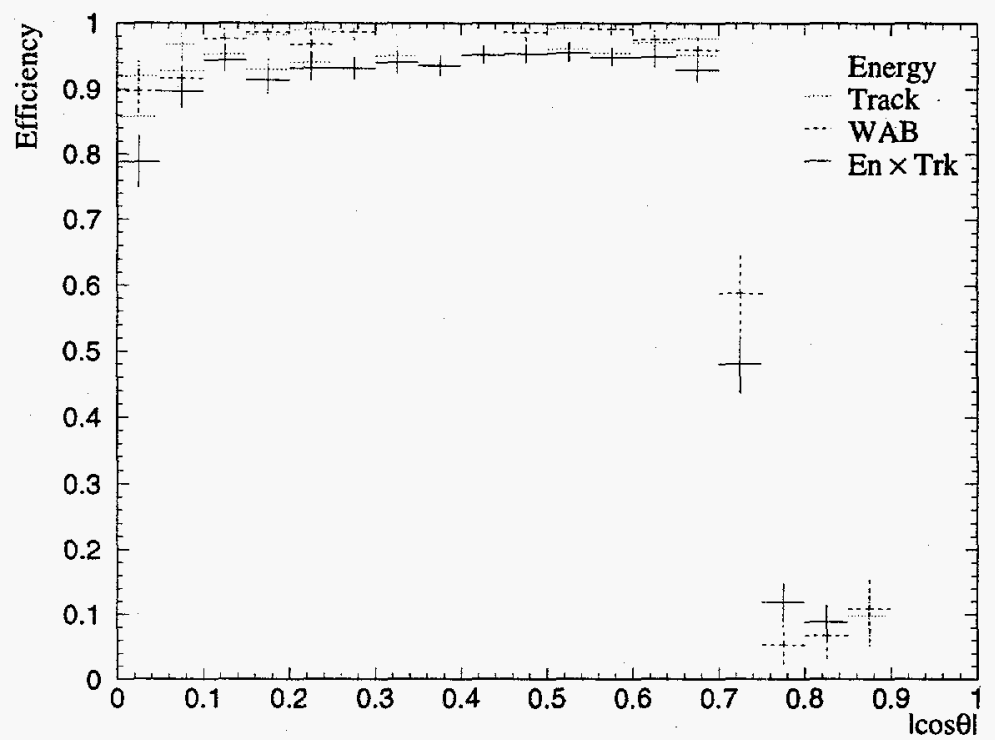

Figure 3.6: Hadron trigger efficiency estimates from correlations.

Similar correlation studies with the Monte Carlo indicate that the correlations give a good measure of the various individual trigger efficiencies. This is not a guarantee that the procedure is correct, but the tracking and calorimetry based triggers should interact in similar way between the data and Monte Carlo in spite of the latter's failings in correctly simulating the charge division for the tracking based triggers.

\subsubsection{Trigger Fractions in the Data}

By examining the fraction of each trigger in the data, we can put an upper bound on efficiency of each trigger. We can also get some idea of how good the overall trigger efficiency is by comparing the trigger efficiency estimates with the overall trigger fractions in the data. From this, it appears that the trigger efficiency in the barrel region is very close to $100 \%$ as the estimated efficiencies follow the trigger fractions quite closely. In the endcap region, it appears that we get the vast majority of our events from the energy trigger. 

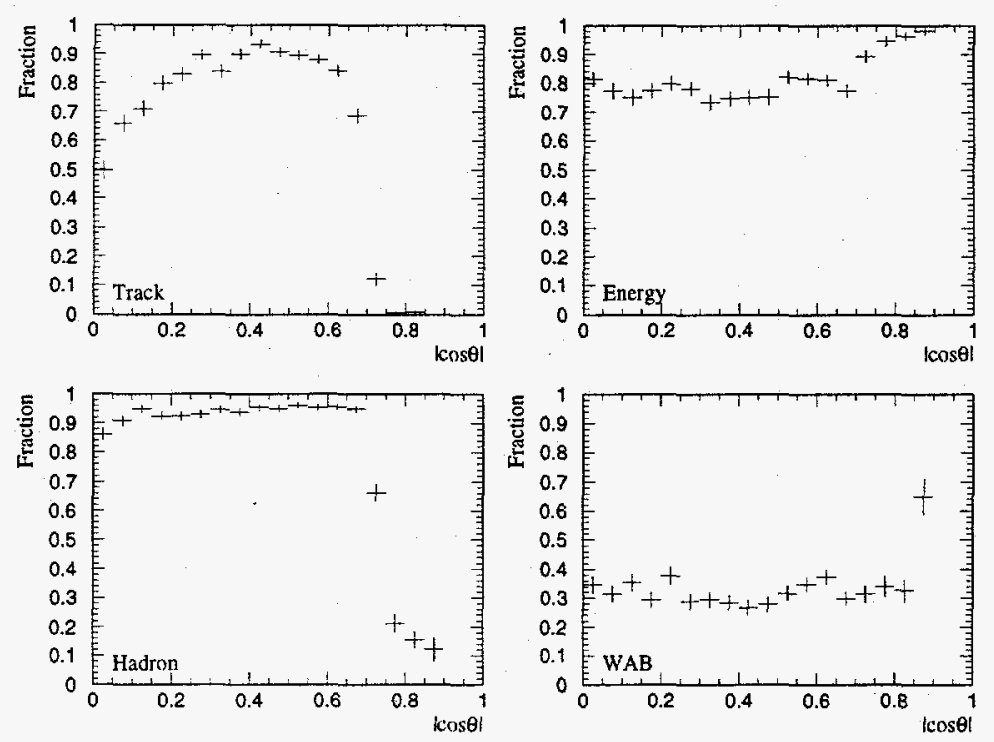

Figure 3.7: Trigger fractions in the data.

\subsection{Data Processing and Reconstruction}

After the data is written to tape, it must be processed to allow physics analysis. This processing, called reconstruction, is quite compute time intensive as detailed fits to tracks must be performed along with the many other calculations needed to get useful physics information from the raw data. The trigger reduces the data rate to a level manageable by the data acquisition system, but this is still too much data to be easily fully reconstructed. As a result, the data are further filtered before full reconstruction is done. In this process, a more detailed pattern recognition routine is run on the CDC data, and some more detailed calorimeter quantities are calculated. To be further processed, an event must pass one of two filters, the pass 1 tau filter or the "EIT"1 pass 1 filter. The pass 1 tau filter requires that there be at least one track with momentum greater than $1 \mathrm{GeV}$. The "EIT" pass 1 filter utilizes several calorimeter quantities:

1. Energy Imbalance Trigger. 
- NEMHI - the number of LAC EM towers with a signal above the "Hi" threshold of 60 ADC counts.

- EHI - the sum of the energy deposited in all of the LAC EM(HAD) towers with a signal greater than the "Hi" threshold of 60(120) ADC counts.

- ELO - the sum of the energy deposited in all of the LAC EM(HAD) towers with a signal greater than the "Low" threshold of 8(12) ADC counts.

The filter requires that

- $\mathrm{NEMHI} \geq 10$

- $\mathrm{EHI}>15 \mathrm{GeV}$ (Min-I scale)

- ELO $<140 \mathrm{GeV}(\mathrm{Min}-\mathrm{I}$ scale)

-2•EHI $>3(\mathrm{ELO}-70 \mathrm{GeV})$

- NEMHI $>0$ in both the north and south hemispheres.

As far as taus are concerned, the pass 1 tau filter is the only pass 1 filter necessary, but the "EIT" pass 1 filter is necessary for other physics. Events that pass these filters are fully reconstructed and made available for physics analysis. The SLD tau filter (described in the next chapter) is designed to pass only those events that would pass the pass 1 filters, so the efficiency of the SLD tau filter includes any effects due to the pass 1 filters.[27] 


\section{Chapter 4}

\section{The Tau Selection}

At this point, the data consists of nearly all of the $\mathrm{Z}^{0}$ events produced during the run as well as various other background events. Most of these events are not tau-pair events, so to make analysis easier, we make a further event selection to isolate a sample consisting mostly of tau-pair events. Having selected these events, it is useful to know how efficiently we are selecting them and how many non-tau events contaminate the sample.

\subsection{Selecting Events}

At the $\mathrm{Z}^{0}$ resonance, tau-pair events are quite distinctive. They have far lower charged and neutral particle multiplicities than typical hadronic $\mathrm{Z}^{0}$ boson decays. Also, they include neutrinos, which allow them to be distinguished from other leptonic $\mathrm{Z}^{0}$ boson decays due to missing energy. Furthermore, they have more energy than two photon events and various machine related backgrounds.

\subsubsection{Track and Cluster Selection}

In order to select taus from the reconstructed data, we must first determine which reconstructed tracks and clusters of calorimeter hits to use. Some tracks and clusters are spurious and others are the result of known background processes, so we must come up with some criteria for the tracks and clusters we use.

One known source of background clusters is SLC muons. These are created in beam interactions with collimators far upstream of the IP and follow the beam to the detector. There are several toroid magnets around the beamline intended to deflect these muons 
away from the detector and for the most part these work. Nonetheless, many events have several muons running parallel to the beamline through the calorimeter. Fortunately, part of the SLD reconstruction is a package designed to identify these muons, which leave a distinctive string of minimum ionizing clusters in the calorimeter. These clusters are tagged as SLC muon clusters, and we ignore them in the analysis. Another source of background clusters is noise from various sources. To eliminate this, we require that a cluster have at least a small amount of energy in LAC EM1 $(10 \mathrm{MeV})$ and LAC EM2 (20 MeV). In particular, this eliminates single hits, or clusters with only a single hit in the calorimeter. We also require that the overall cluster energy be greater than $100 \mathrm{MeV}$. All of these energies are in the so-called minimum ionizing scale. Some clusters are associated with tracks by the reconstruction. A cluster is associated with a track if the extrapolation of the track to the EM1 section of the LAC hits any of the EM1 towers that are included in the cluster: These are only used by the analysis in conjunction with the associated track.

Background tracks can come from several sources. Photons can convert in the beam pipe or VXD to an electron positron pair. We use a standard SLD software package to identify and flag pairs of oppositely charged tracks that are consistent with being produced by a conversion. These tracks are ignored by the analysis, but the track momenta of each pair are combined and treated by the analysis as a neutral cluster. Cosmic rays and beam gas interactions can also lead to spurious tracks. These are eliminated by requiring that all tracks used in the analysis originate from within $6 \mathrm{~cm}$ of the IP in $\mathrm{z}$ and $3 \mathrm{~cm}$ of the IP in radius. To eliminate poorly fit tracks, we require that each track used in the analysis have at least $20 \mathrm{CDC}$ hits and that the quantity $\sqrt{2 \chi^{2}}-\sqrt{2 \mathrm{n}-1}$ be less than 10 for the track fit, where $\mathrm{n}$ is the number of degrees of freedom for the fit. We also require that all tracks have a transverse momentum $\mathrm{p}_{\mathrm{t}}>100 \mathrm{MeV}$. 


\subsubsection{Tau Selection Quantities}

To select taus, we must calculate certain quantities for each event. To start, we divide the event into two tau hemispheres. As a starting point, we use the highest momentum track in the event. We then use an iterative procedure to build up two "jets." Using the momentum of the largest track as a seed direction, we sum up the momenta of all tracks and clusters within $15^{\circ}$ of the seed direction to give a new seed direction. We repeat this procedure until the jet direction does not change or we have gone through 24 iterations. For tau events, this procedure generally goes through a small number of iterations. We define the momentum of a cluster by assuming that it represents a massless particle traveling from the IP to the centroid of the cluster with energy equal to the cluster's deposited energy. To build the second jet, we use the vector sum of all track momenta not included in the first jet as the initial seed direction and repeat the above iterative procedure. Once the total charges for the two jets have been calculated, we rename the jets so that jet $\mathbf{1}$ is the negatively charged jet, or if both have the same charge, it is the jet with the highest momentum track.

We then categorize all the tracks and clusters in the event in three classes, those belonging to jet 1 , those belonging to jet 2 and those outside jets. Tacks and clusters within $15^{\circ}$ of the jet directions are considered to belong to the appropriate jet, and all others are considered to be outside jets. We then calculate various quantities for the event and each jet. Jet and event invariant masses are calculated assuming that the tracks represent pions and the clusters not associated with tracks represent photons. We define visible energy as the scalar sum of all track momenta and unassociated cluster energies for the given category. The missing momentum for an event is defined as the opposite of the vector sum of all track momenta and unassociated cluster energies in the event. The EM LAC energy for the event is calculated using the corrected EM scale energy (see Appendix E) for each 
cluster. Acolinearity angles are calculated between the two jet directions and between the two tracks in 1-1 (meaning one track in each jet or hemisphere) events. We define the acolinearity between two vectors as the angle between one vector and the opposite of the other vector.

\subsubsection{Tau Selection Cuts}

We call an event a tau pair event if it has:

1. at least one track in each hemisphere

2. at least one jet with $|\Sigma Q|=1$

3. total visible energy $>10 \mathrm{GeV}$

4. number of tracks in jets $<7$

5. number of unassociated clusters in jets $<9$

6. no tracks outside jets

7. total visible energy outside jets $<5 \mathrm{GeV}$

8. the acolinearity angle between the jets $<20^{\circ}$

9. all jets with more than one track have invariant mass $<2.3 \mathrm{GeV}$.

10. for $1-1$ events, the acolinearity angle between the tracks $>0.573^{\circ}(10 \mathrm{mrad})$

11. scalar sum of the two largest track momenta $<65 \mathrm{GeV}$

12. total EM LAC energy (corrected) in the event $<62.5 \mathrm{GeV}$

13. $\left|\cos \theta_{\text {miss }}\right|<0.88$

Cuts 1 and 2 are designed to make sure we have a useful event and that we can distinguish it as a tau pair. For the moment all of our tau analyses are strongly track based, so it is useful to require that the selected events fall within the tracking volume. Also, the most distinctive signature of tau pair events is their low track multiplicity. As a result, an efficient and pure tau filter should be track based. There has been some work on extending the tau filter to the endcap regions, but due to the lack of useful tracking there, this has been 
largely unsuccessful. Cut 3 rejects junk events such as beam gas events and ensures that no events that would fail the pass 1 filters can pass the tau filter, thus allowing Monte Carlo studies to be done without running both the tau and pass 1 filters. This cut also is quite effective at rejecting two photon events which generally have low visible energy due to losses along the beampipe. Cuts 4 and 5 reject multihadronic final states by restricting the track and cluster multiplicities in jets. We cut on clusters in jets because that quantity is less sensitive to SLC muon background than the total number of clusters in the event. Cuts 6 and 7 also reject multihadronic final states because these states generally have fatter jets than tau pair events and can have more than two jets. The total energy outside jets is much less sensitive to SLC muons than the number of clusters as SLC muons produce minimum-ionizing clusters, which have relatively low energy. Cut 8 also rejects multihadronic final states, particularly those that lose some tracks or a jet in the endcap region. It also rejects two photon events, which tend to have high acolinearities between the jets that they do produce. Cut 9 rejects multihadronic events by requiring that the jets have masses consistent with being a tau. Cuts 10 and 11 reject both WAB and mu-pair events by eliminating highly back to back pairs of tracks and high momentum pairs of tracks. Cut 12 rejects WAB events, including radiative events, due to the preponderance of electromagnetic energy in those events. Cut 13 rejects those events that lose a lot of their energy along the beampipe. Cosmic ray events are rejected by the requirement that tracks come from the IP to be included in the analysis.

Table 4.1: SLD Tau Sample Size.

\begin{tabular}{l|r}
\hline \multicolumn{1}{c|}{ Run Period } & \multicolumn{1}{|c}{ Selected Taus } \\
\hline 1993 & 1308 \\
\hline 1994 Summer & 548 \\
\hline 1994 Fall & 1510 \\
\hline 1995 & 962 \\
\hline
\end{tabular}




\subsubsection{Plots of Tau Filter Quantities for Monte Carlo and Data}

Following are plots of the 11 quantities relevant to the tau filter (Fig. 4.1 through Fig. 4.11). The plots are for MC taus and backgrounds normalized to the overall 1993-5 luminosity. The plots on the left are before all cuts, and those on the right are after all cuts but those on the relevant quantity. The distributions from the 1993-5 data after the tau filter are superimposed for comparison. The relevant cuts are shown on the plots with vertical line(s). For the Monte Carlo, we generate events and run them through a GEANT[26] based simulation of the SLD. To this simulated data for each event, we add the detector read out from a random trigger event from a sample of random events selected to be representative of typical machine backgrounds for the selected running period. This allows proper simulation of machine based backgrounds, which are not well modeled. The simulated data from the simulated events (with random overlays) are then run through the standard SLD reconstruction. The generated event information is retained with the reconstructed simulated data.

We generate mu and tau pairs using the KORALZ event generator[25], which accurately simulates tau and mu pair production at the $\mathrm{Z}^{0}$ resonance over its full allowable kinematic range. The WABs are generated with the UNIBAB event generator[28], with a suitable angular cutoff well outside the tracking acceptance of the SLD. This cutoff is necessary due to the singular nature of the WAB cross section at high $\cos \theta$, which makes it impossible to simulate all allowed kinematics. The multihadrons are generated using Lund 7.4[29], and the two photon events are generated using a modification of the MAC two photon event generator[30], again with an angular cut off well outside the SLD tracking region. For the two photon processes, we actually generate four different processes and combine them, correctly weighted for cross section. These processes correspond to the two photons fusing to form either a pair of electrons, muons, taus, or quarks. 
There are some discrepancies between the Monte Carlo and the data which are due to the fact that the Monte Carlo samples are generated simulating the conditions during the 94 fall running period. This period had relatively high rates of SLC muons, which results in higher numbers of low energy clusters than we see in the overall data sample. Also, the Monte Carlo assumes that the energy trigger is fully read out, which is not true for the first half of the data. As a result, the Monte Carlo predicts an excess of events in the region above $|\cos \theta|=0.7$. We have run tau Monte Carlo samples for the other relevant running periods, and tau filter is relatively unaffected by the differences in machine backgrounds. Unfortunately, we have not generated background samples for all relevant running periods so the following plots are for the ' 94 fall period where we have generated the requisite samples.
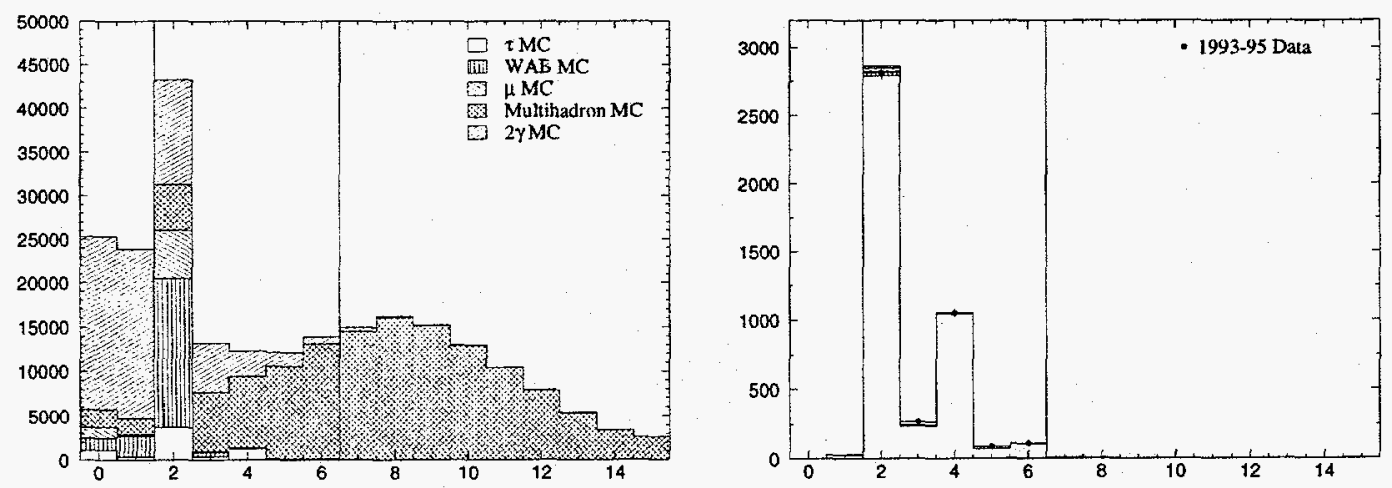

Figure 4.1: Overall number of tracks. 

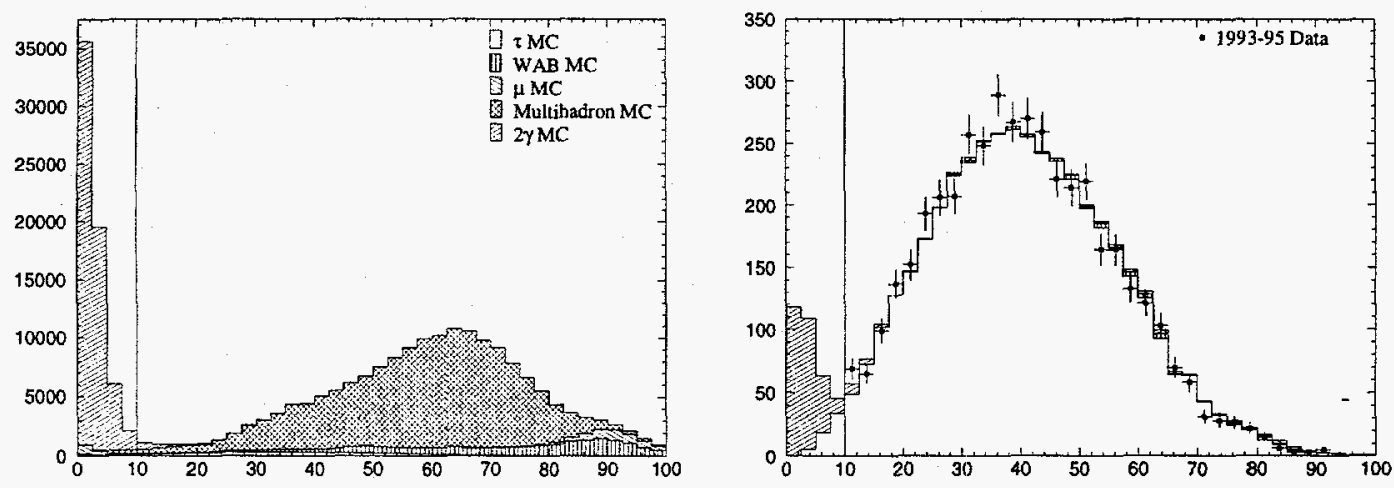

Figure 4.2: Total visible energy. (GeV)
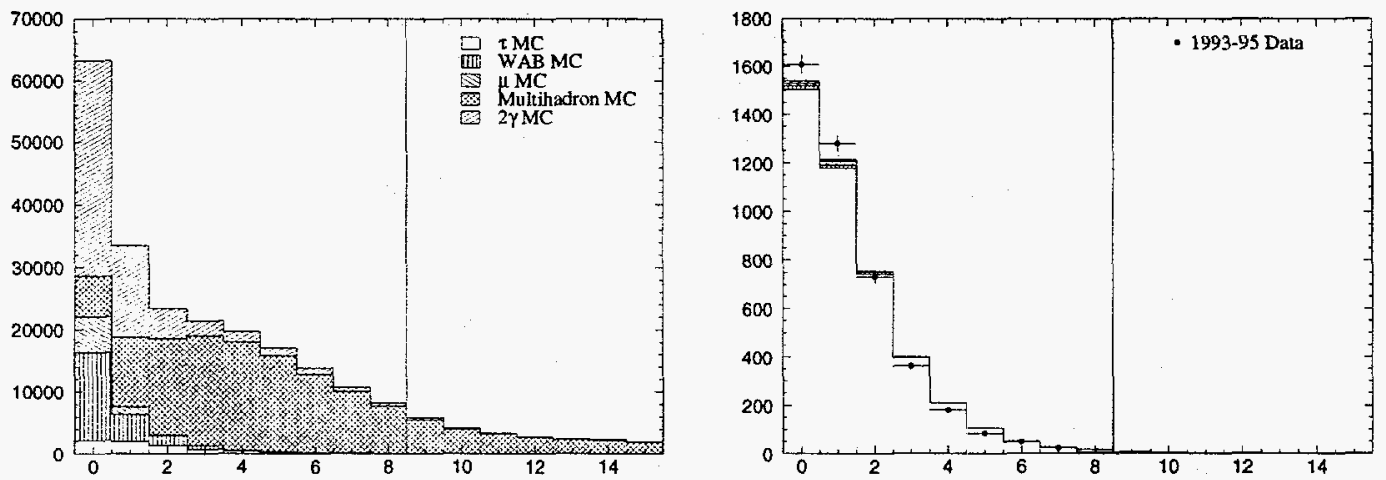

Figure 4.3: Number of unassociated clusters in jets.
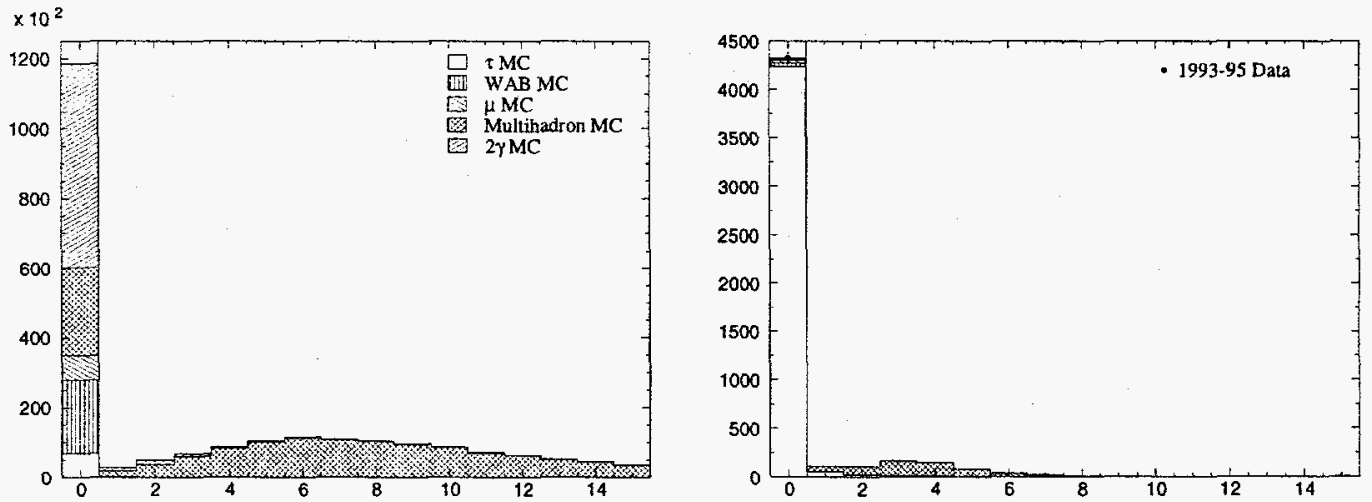

Figure 4.4: Number of tracks outside jets. 

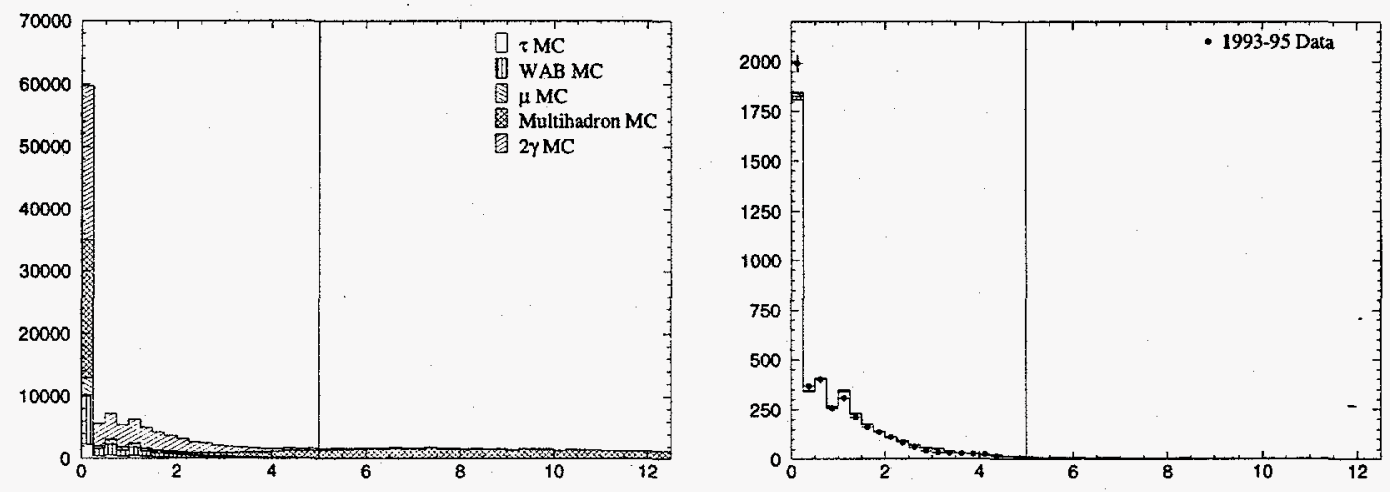

Figure 4.5: Energy outside jets. (min-I GeV)
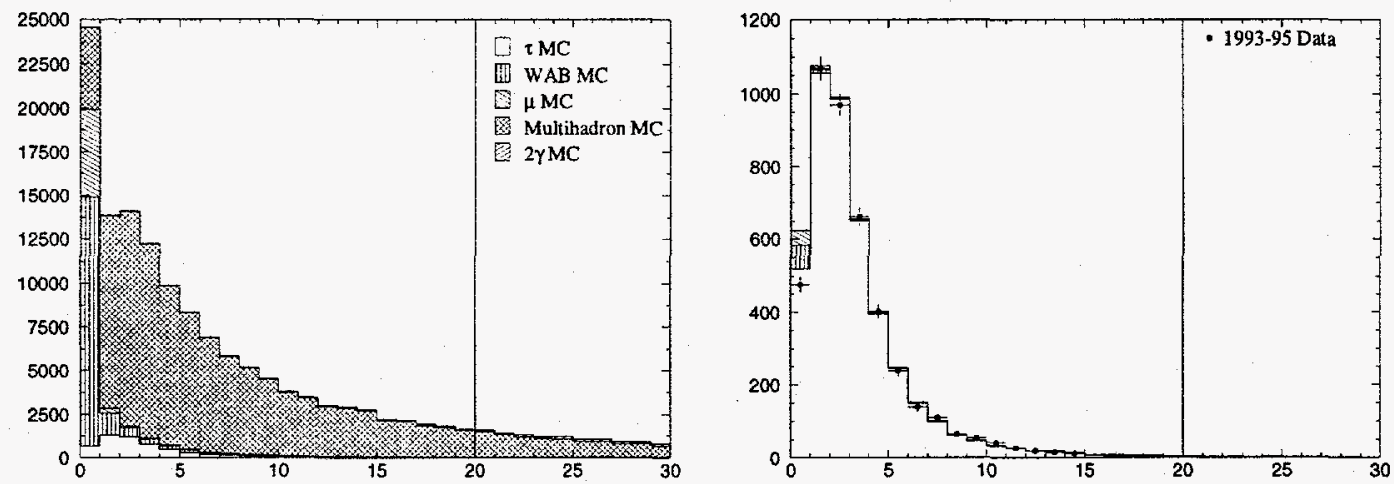

Figure 4.6: Event acolinearity. $\left({ }^{\circ}\right)$
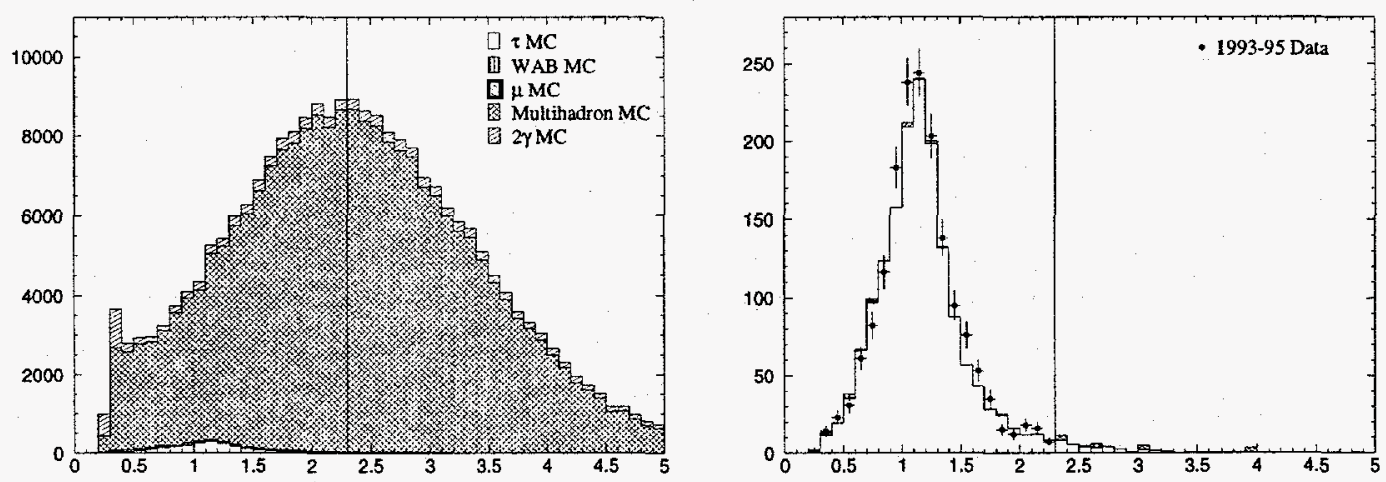

Figure 4.7: Multiprong jet mass. (GeV) 

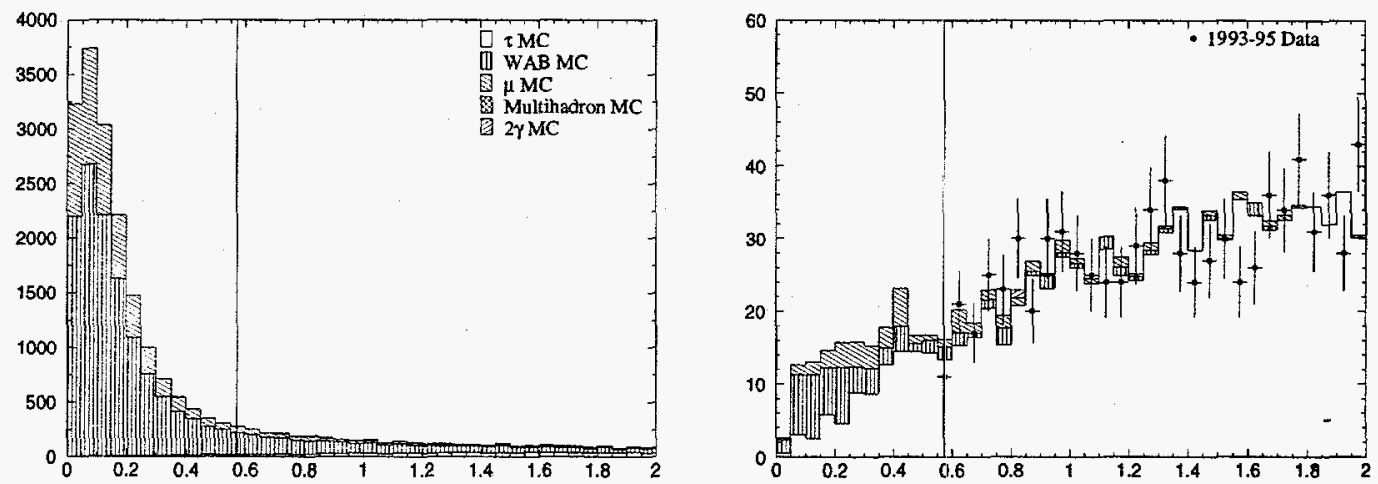

Figure 4.8: 2 prong acolinearity. $\left({ }^{\circ}\right)$
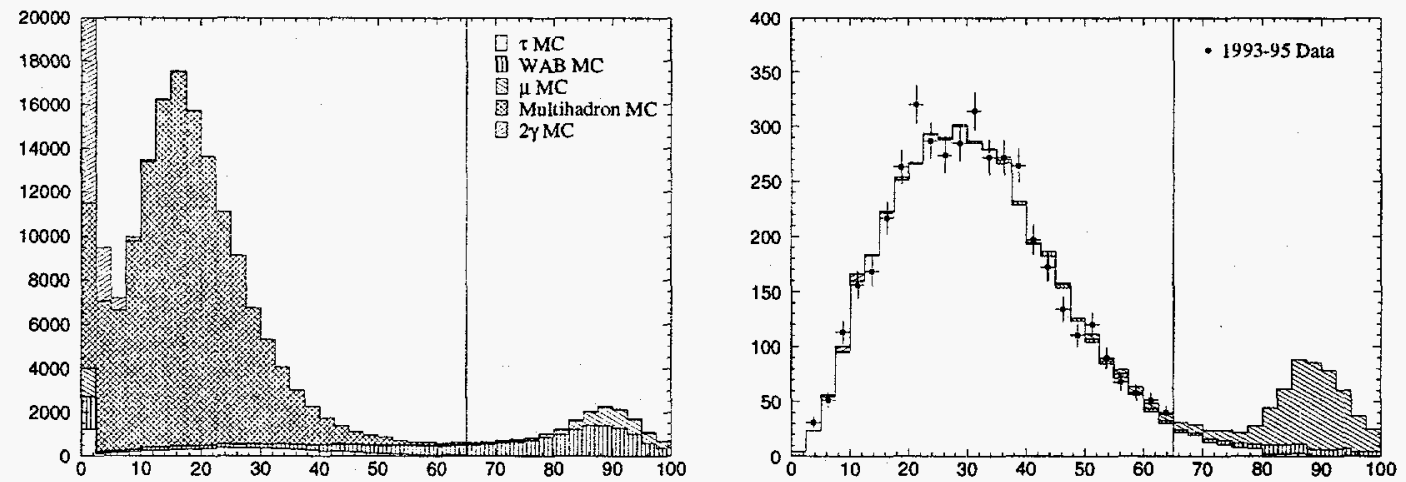

Figure 4.9: Scalar sum of two largest track momenta. (GeV)
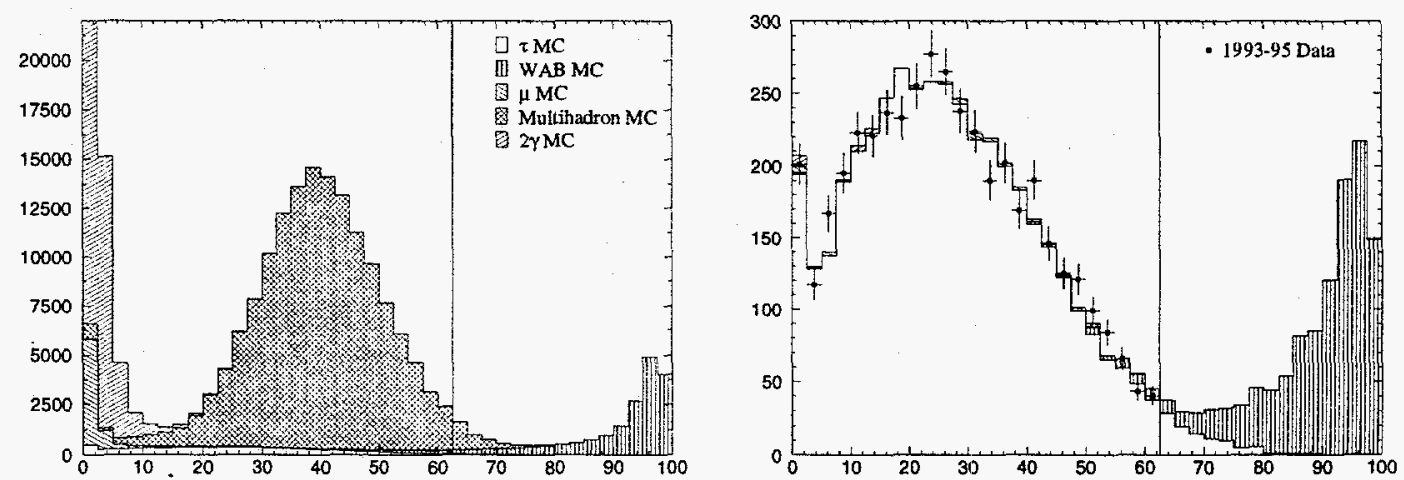

Figure 4.10: Total electromagnetic energy. (corrected GeV) 

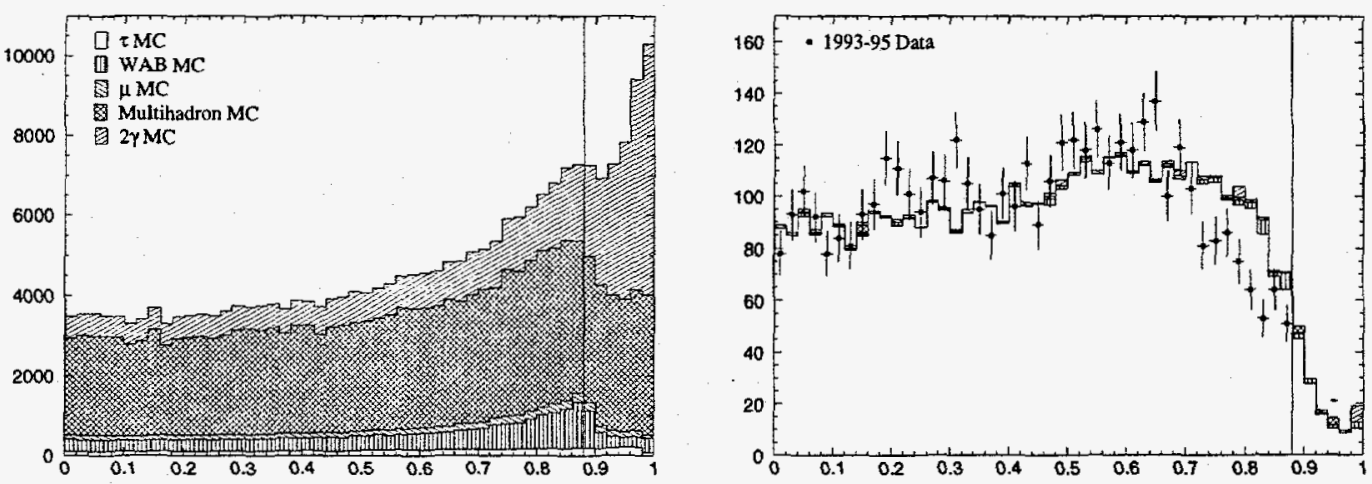

Figure 4.11: Missing momentum. (I $\left.\cos \theta_{\text {miss }} l\right)$

\subsection{Efficiency and Backgrounds for the Tau Filter}

We can use the Monte Carlo samples to estimate the selection efficiency of the tau filter and the amount of background that it allows to pass. As a cross check, we can compare the number of selected taus that the efficiency predicts with the number of taus that we actually select from the 1993-95 data.

\subsubsection{Tau Selection Efficiencies from Monte Carlo}

Using the samples of tau Monte Carlo events, we can estimate the overall tau filter efficiency as well as the dependency of the efficiency on various quantities such as angle or decay type. These efficiencies are determined by counting the fraction of a set of generated events that meet a given criterion pass the tau filter.

$$
\varepsilon_{\text {Estimated }}=\frac{\mathrm{N}_{\text {Pass }}}{\mathrm{N}_{\text {Generated }}}
$$

For these efficiencies we also fold in a trigger requirement to get an idea of the overall efficiency for selecting tau events. Due to the failings of the trigger simulation in the central region of the detector, we assume that the trigger efficiency is $100 \%$ for $|\cos \theta|<0.4$ and use 
the trigger simulation outside this region. This seems to be al good assumption as the trigger correlation studies seem to indicate that trigger efficiencies are near $100 \%$ in the barrel. For the 1993 and 1994 summer running periods, we remove the energy trigger from the trigger requirement as the full detector was not read out for these periods. This results in Fig. 4.12 for the 1994 fall running period.

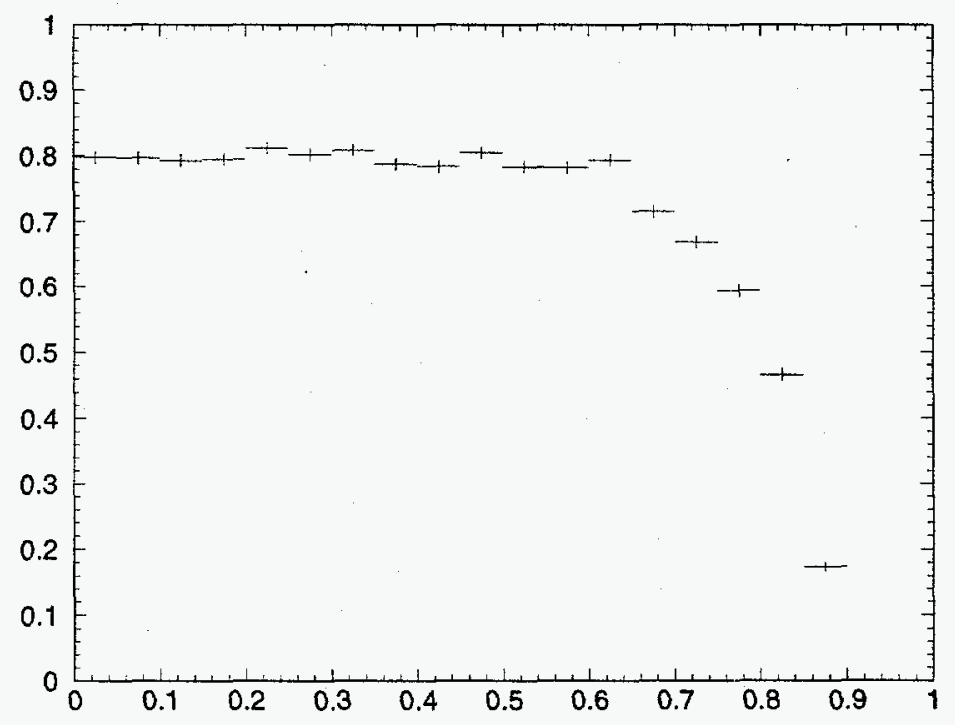

Figure 4.12: Tau selection efficiency vs. $|\cos \theta|$ from MC.

This shows that selection efficiency is generally flat at about $80 \%$ in the barrel region and falls off at high $\cos \theta$ where the useful tracking region of the SLD ends. The overall tau trigger and selection efficiency comes to about 0.60 with the energy trigger read out and 0.52 without the energy trigger read out.

Table 4.2: Tau Selection Efficiency. (no Energy Trigger)

\begin{tabular}{l|r|r|r}
\hline . Run Period & Events Generated & Events Selected & Efficiency \\
\hline 1993 & 47043 & 24794 & 0.53 \\
\hline 1994 Summer & 18967 & 9710 & 0.51 \\
\hline Total & 66010 & 34504 & 0.52 \\
\hline
\end{tabular}


Table 4.3: Tau Selection Efficiency. (with Energy Trigger)

\begin{tabular}{l|r|r|r}
\hline \multicolumn{1}{c|}{ Run Period } & Events Generated & Events Selected & Efficiency \\
\hline 1994 Fall & 39238 & 23473 & 0.60 \\
\hline 1995 & 29844 & 18066 & 0.61 \\
\hline Total & 69082 & 41539 & 0.60 \\
\hline
\end{tabular}

Since we have the full generated event information, we can break this efficiency down by decay type (as in 3.2.1) or decay topology (Table 4.4 and Table 4.5). Here we report the efficiencies for the full energy trigger read out.

Table 4.4: Tau Selection Efficiency by Topology from 94 Fall MC.

\begin{tabular}{c|r|r|r}
\hline Topology & Generated & Selected & Efficiency \\
\hline $1-1$ & 28529 & 16811 & 0.59 \\
\hline $1-3$ & 9786 & 6145 & 0.63 \\
\hline $1-5$ & 58 & 37 & 0.64 \\
\hline $3-3$ & 853 & 478 & 0.56 \\
\hline $3-5$ & 12 & 2 & 0.17 \\
\hline
\end{tabular}

Table 4.5: Tau selection Efficiency for Events Including Given Decays.

\begin{tabular}{l|r|r|r}
\hline Decay & Generated & Selected & Efficiency \\
\hline $\mathrm{e}$ & 14102 & 8412 & 0.60 \\
\hline$\mu$ & 13589 & 7956 & 0.59 \\
\hline$\pi$ & 9355 & 5524 & 0.59 \\
\hline$\pi \pi^{0}$ & 28140 & 16872 & 0.60 \\
\hline $3 \pi$ & 11504 & 7103 & 0.62 \\
\hline other & 1786 & 1079 & 0.60 \\
\hline
\end{tabular}




\subsubsection{Backgrounds from the Monte Carlo}

Using the known cross sections for the various background processes and the number of events from the Monte Carlo background samples that pass the tau filter, we can estimate the background contamination in the tau sample. In most cases, the production cross sections are well known; however in the case of the two photons, the generator cross sections are not particularly reliable, but the corresponding background is small, and the data show no indication of anomalous backgrounds.

Table 4.6: '94 Fall Monte Carlo Samples.

\begin{tabular}{l|r|r|r|r|r|r}
\hline \multicolumn{1}{|c|}{ Process } & $\sigma\left(\mathrm{nb}^{-1}\right)$ & $\begin{array}{c}\text { Generated } \\
\text { events }\end{array}$ & $\begin{array}{r}\text { Generated } \\
\text { Lum. }\left(\mathrm{nb}^{-1}\right)\end{array}$ & $\begin{array}{c}\text { Selected } \\
\text { events }\end{array}$ & Sel./ Lum. & $\begin{array}{c}\text { Frac. } \\
(\%)\end{array}$ \\
\hline$\tau^{+} \tau^{-}$ & 1.467 & 39238 & 26747 & 23473 & 877.6 & 97.86 \\
\hline WAB & 4.361 & 36206 & 8302 & 65 & 7.8 & 0.87 \\
\hline$\mu^{+} \mu^{-}$ & 1.470 & 20453 & 13914 & 85 & 6.1 & 0.68 \\
\hline multihadron & 30.51 & 153959 & 5046 & 13 & 2.6 & 0.29 \\
\hline $2 \gamma(\mathrm{ee})$ & 2.121 & 19869 & 9368 & 10 & 1.1 & 0.12 \\
\hline $2 \gamma(\mu \mu)$ & 1.865 & 19869 & 10654 & 12 & 1.1 & 0.12 \\
\hline $2 \gamma(\tau \tau)$ & 0.275 & 19869 & 72172 & 19 & 0.3 & 0.03 \\
\hline $2 \gamma(\mathrm{qq})$ & 8.627 & 38938 & 4514 & 1 & 0.2 & 0.02 \\
\hline
\end{tabular}

Table 4.6 shows the various Monte Carlo samples that we have generated for the ' 94 fall SLD running period. From this information, we expect that the SLD tau sample has a little over $2 \%$ background contamination, mostly from wide angle Bhabha and mu-pair events. Inspection of the actual selected tau data seems to bear this out.

\subsubsection{Comparison of Efficiency and Background to the Data}

We have several methods to determine the total luminosity for the data. Using these, it is possible to estimate the number of taus we expect to select. The most straightforward estimate of the luminosity is the number measured by the luminosity monitor. We can also 
estimate luminosity using the numbers of various types of events, including multihadronic $\mathrm{Z}^{0}$ boson decays and mu-pairs, and the known cross sections and estimated filter efficiencies for them.

Using the luminosity measured by the SLD luminosity monitor and the tau pair cross section, we can estimate the expected number of selected tau events for the different SLD running periods.

$$
N_{S e l} \sim \frac{\varepsilon_{\tau}\left(\mathcal{L} \cdot \sigma_{\tau}\right) f_{C D C}}{\pi_{\tau}}
$$

According to a Monte Carlo calculation using the KORALZ Monte Carlo program, the cross section for producing tau pairs $\left(\sigma_{\tau}\right)$ is $1.467 \mathrm{nb}$ at the nominal SLC collision parameters. We must correct the raw number of taus from the cross section for the selection efficiency $\left(\varepsilon_{\tau}\right)$ and background $\left(\pi_{\tau}\right.$ the purity of the selected tau pairs which is 0.979$)$. In addition, the luminosity numbers are for the entire run period when the polarimeter was giving valid polarization measurements. This period was generally identical to the period when the beams were at or near nominal polarization. Since the tau filter itself makes no polarization requirement, to check the estimates, we must exclude those events with small or zero beam polarizations (lpolk<0.4). In addition, during running there are periods when due to beam backgrounds, the CDC must be turned off or run at reduced voltage. The integrated luminosity measurement includes these periods, but it is impossible to select taus due to the lack of tracking. As a result, we must correct the estimate for the CDC duty factor $\left(f_{C D C}\right)$. Unfortunately, we don't have an accurate evaluation of the CDC uptime for the 1993 run other than an anecdotal $95 \%$. The resulting estimates are summarized in Table 4.7. 
Table 4.7: Estimated Taus from Measured Luminosity.

\begin{tabular}{l|r|r|r|r|r}
\hline Run Period & $\int \mathcal{L}\left(\mathrm{nb}^{-1}\right)$ & $\varepsilon_{\tau}$ & $\mathrm{f}_{\mathrm{CDC}}$ & Est. $\tau \mathrm{s}$ & $\tau \mathrm{s}$ w/ Pol. \\
\hline 1993 & 1781 & 0.53 & $\sim 0.95$ & 1344 & 1274 \\
\hline 1994 Sum. & 628 & 0.51 & 0.95 & 454 & 440 \\
\hline 1994 Fall & 1822 & 0.60 & 0.95 & 1556 & 1464 \\
\hline 1995 & 1121 & 0.61 & 0.96 & 984 & 951 \\
\hline
\end{tabular}

We can also compare the number of taus selected with the total number of hadronic $\mathrm{Z}^{0}$ decays selected for the $A_{L R}$ analysis. This is a well studied event sample that should give a good estimate of luminosity and hence the number of tau pairs we expect to select. Actually, several different filters have been used for the $A_{L R}$ analysis. This comparison will be done with the $\mathrm{KAL} \mathrm{Z}^{0}$ filter [31], which is consistent over all running periods.

$$
\mathrm{N}_{\mathrm{Sel} \tau} \sim\left(\frac{\mathrm{N}_{\mathrm{KAL}} \cdot \pi_{\mathrm{KAL}}}{\varepsilon_{\mathrm{KAL}}}\right) \cdot\left(\frac{\mathrm{BR}\left(\mathrm{Z} \rightarrow \tau^{+} \tau^{-}\right)}{\mathrm{BR}(\mathrm{Z} \rightarrow \text { hadrons })}\right) \cdot\left(\frac{\varepsilon_{\tau}}{\pi_{\tau}}\right) \cdot \mathrm{f}_{\mathrm{CDC}}
$$

These events are required to have near nominal polarizations, so again we must compare to taus with near nominal polarizations. To estimate the number of taus from the number of hadronic $\mathrm{Z}^{0} \mathrm{~s}$ we must use the $\mathrm{Z}^{0}$ branching ratios: $\mathrm{BR}$ (hadronic) $=0.6990 \pm 0.0015$ and $\operatorname{BR}\left(\tau^{+} \tau^{-}\right)=0.03360 \pm 0.00015$.[7] Because the $\mathrm{KAL} \mathrm{Z}^{0}$ filter uses only the calorimetric subsystems of the SLD, we will again have to correct for the CDC duty factor.

Table 4.8: Estimated Taus from KAL $Z^{0}$ s.

\begin{tabular}{l|r|r|r|r|r}
\hline Run Period & $\mathrm{N}_{\mathrm{KAL}}$ & $\varepsilon_{\mathrm{KAL}}$ & $\pi_{\mathrm{KAL}}$ & \multicolumn{1}{c|}{ Est. $\tau \mathrm{s}$} & \multicolumn{1}{c}{$\tau_{\mathrm{s} w / P o l .}$} \\
\hline 1993 & 49392 & 0.94 & 0.998 & 1296 & 1274 \\
\hline 1994 Sum. & 17373 & 0.94 & 0.998 & 439 & 440 \\
\hline 1994 Fall & 49169 & 0.94 & 0.998 & 1461 & 1464 \\
\hline 1995 & 31105 & 0.94 & 0.998 & 949 & 951 \\
\hline
\end{tabular}


From these estimates, it appears that the luminosity measurements may be a little high. It also appears that we have a good estimate of the tau selection efficiency and backgrounds. 


\section{Chapter 5}

\section{Selection of Leptonic Tau Decays}

Now that we have a nice clean sample of tau pair events, we must select the leptonic final states in order to do the analysis. Fortunately, leptonic tau decays are fairly distinct from other tau decays. Electronic decays are characterized by a lot of electromagnetic energy, and muonic decays are characterized by deep penetration of the SLD.

\subsection{Selection Quantities and General Requirements}

Leptonic tau decays are one-prong decays, therefore to start we select only those hemispheres with a single track as defined for the tau filter. In addition, we require that the track have at least one hit in the vertex detector associated with it. This requirement significantly improves momentum resolution as the VXD hit gives an additional high resolution point on the track close to the IP. This requirement effectively limits the angular region for these events, but to ensure a clean cut off in angle we further require that the track have $|\cos \theta|<0.75$. For the purposes of the analysis, we further restrict the scaled energy

$\left(x=\frac{2 p}{E_{C M}}\right)$ to fall within the range from 0.035 to 1 (roughly $1.6 \mathrm{GeV}<\mathrm{p}<45.6 \mathrm{GeV}$ ). We then use several hemisphere and track quantities to select the various decays:

ESTAT - a quantity calculated by the SLD reconstruction, it is based on ratio of the track momentum and the energy of its associated cluster and the width of the associated cluster. ESTAT is an integer quantity where values $0,1,2$, and 3 indicate "Platinum", "Gold", "Silver", and "Bronze" as the likelihood of the track being an electron, which corresponds to how close $\mathrm{E} / \mathrm{p}$ and the shower spread are to that expected from an electron. 
MUSTAT - a quantity calculated by the SLD reconstruction, it is based on association of WIC hits with the extrapolated track direction and the penetration indicated by the WIC hits. MUSTAT is an integer quantity where values $0,1,2$, and 3 indicate "Platinum", "Gold", "Silver", and "Bronze" as the likelihood of the track being a muon. Basically "Platinum" corresponds to the track being the only track the matches a set of WIC hits that has at least two hits in the outer four layers of the WIC (penetration). "Gold" is similar, but the track is the best match to such a set of WIC hits. "Silver" and "Bronze" are similar, but without penetration.[23]

$\mathrm{E} / \mathrm{p}$ - the ratio of the associated cluster energy in the minimum ionizing scale to the measured track momentum.

$\mathrm{N}_{\text {tow }}$ - the number of towers with hits in the EM sections of the LAC that are included in the cluster associated with the track.

Neutrals - clusters not associated with the track, but within $15^{\circ}$ of the track direction.

Jet Mass - the invariant mass of everything within $15^{\circ}$ of the track direction, assuming the track has the pion mass and the neutrals are photons.

Pseudo Mass - the invariant mass of the track and its associated cluster assuming that the track has the pion mass and the associated cluster is a photon.

CRID $\mathcal{L}(\mathrm{e})-\mathcal{L}(\pi)$ - the difference between the calculated log likelihood that a track is an electron and the log likelihood that it is a pion based on CRID information.

HAD2 - the amount of energy deposited in the HAD2 section of the LAC for the cluster associated with the track.

\subsubsection{Muon Identification Requirements}

We divide the muon selection into two angular regions. Since the SLC muons parallel the barrel WIC Iarocci tubes, we can successfully use it to identify muons. In the endcaps, the 
SLC muons are perpendicular to the Iarocci tubes, so they can easily fake a muon signature because they penetrate the endcap WIC; so we must use shower shapes to identify muonic tau decays in this region $(|\cos \theta|>0.62)$. In the barrel region we call a track a muon if it has:

- MUSTAT $<2$

For tracks with $|\cos \theta|>0.62$, we require:

- $\mathrm{E} / \mathrm{p}<0.3$

- $\mathrm{N}_{\text {tow }}<4$

- No Neutrals

- Pseudo Mass < $180 \mathrm{MeV}$.

We expect that the biggest background to the muons will be pionic tau decays. MUSTAT is quite effective in the barrel region. In the endcap region, we use $\mathrm{E} / \mathrm{p}$ to eliminate electrons. The cut on the number of towers eliminates those pions which interact and shower in the EM section of the LAC, in contrast with muons which will pass through the LAC without showering and hit only one or two towers in each of the two EM sections of the LAC. Also, to reject rho meson decays, we reject tracks with nearby neutrals, and tracks with high pseudo mass.

Table 5.1: SLD Selected Tau to Mu events.

\begin{tabular}{l|r}
\hline \multicolumn{1}{c|}{ Run Period } & \multicolumn{1}{|c}{ IDed Muons } \\
\hline 1993 & 364 \\
\hline 1994 Summer & 143 \\
\hline 1994 Fall & 408 \\
\hline 1995 & 257 \\
\hline Total & 963 \\
\hline
\end{tabular}




\subsubsection{Electron Identification Requirements}

We call a track an electron if all of the following conditions are met:

- It is not identified as a muon.

- ESTAT $<4$ or CRID $\mathcal{L}(\mathrm{e})-\mathcal{L}(\pi)<20$.

- $\mathrm{HAD} 2=0$

$\cdot 3<\mathrm{N}_{\text {tow }}<25$

- No Neutrals and Pseudo Mass $<500 \mathrm{MeV}$ or 1-2 Neutrals and Jet Mass $<500 \mathrm{MeV}$.

The ESTAT and CRID requirements provide a good basis for electron identification. We expect the primary background to this to be tau decays to rho mesons where the photons from the neutral pion from the rho meson decay are merged with the cluster associated with the charged pion track. As a result, we cut on HAD2, which reduces this by rejecting the charged pion which penetrates the LAC more deeply than an electron would. We also cut on the number of towers in the shower to eliminate both muons and rhos. In a merged shower situation, the resulting cluster will tend to be wider than that from a single electron shower. The pseudo mass cut also helps reject rho meson decays. Because electron showers in the LAC tend to be slightly spongy, which can result in them being split into several clusters by the clustering algorithm, we allow tracks that have one or two nearby neutrals to be called electrons; this improves the efficiency by about $10 \%$. In this case, we use the invariant mass cut to guard against contamination from rho meson decays. 
Table 5.2: SLD Selected Tau to Electron events.

\begin{tabular}{l|r}
\hline \multicolumn{1}{c|}{ Run Period } & IDed Electrons \\
\hline 1993 & 326 \\
\hline 1994 Summer & 121 \\
\hline 1994 Fall & 302 \\
\hline 1995 & 214 \\
\hline Total & 963 \\
\hline
\end{tabular}

\subsubsection{Plots of Particle Identification Quantities}

Following are plots for the various quantities used for particle identification (Fig. 5.1 through Fig. 5.6). We have plotted the various quantities for all hemispheres that have one track with a VXD hit and fall within $|\cos \theta|<0.75$. We plot the Monte Carlo simulated data separately for each decay channel as generated and overlay the actual distributions from the 1993-95 selected tau data. The Monte Carlo is normalized to match the number of events in the data. The Monte Carlo does not include non-tau sources of background which are small in any case.

Here we see that we are beginning to reach the limits of the simulation of the detector. From the HAD2 plot (Fig. 5.6) we can see that we are underestimating penetration of the LAC. Studies show that this is mostly due to the modeling of hadronic showers. Electron showers in WABs are well modeled by the Monte Carlo. As a result, the HAD2 cut for electrons is probably slightly more effective at eliminating pions and tho mesons than we would estimate from the Monte Carlo. We also see that for muons in particular, the data seem to have slightly more EM towers (Fig. 5.2) hit than we would expect from the data. Because of this, we used the barrel muons selected by MUSTAT to tune the endcap muon 
cuts. In all cases, we have studied the data distribution for the selected decays as a crosscheck for the identification cuts.

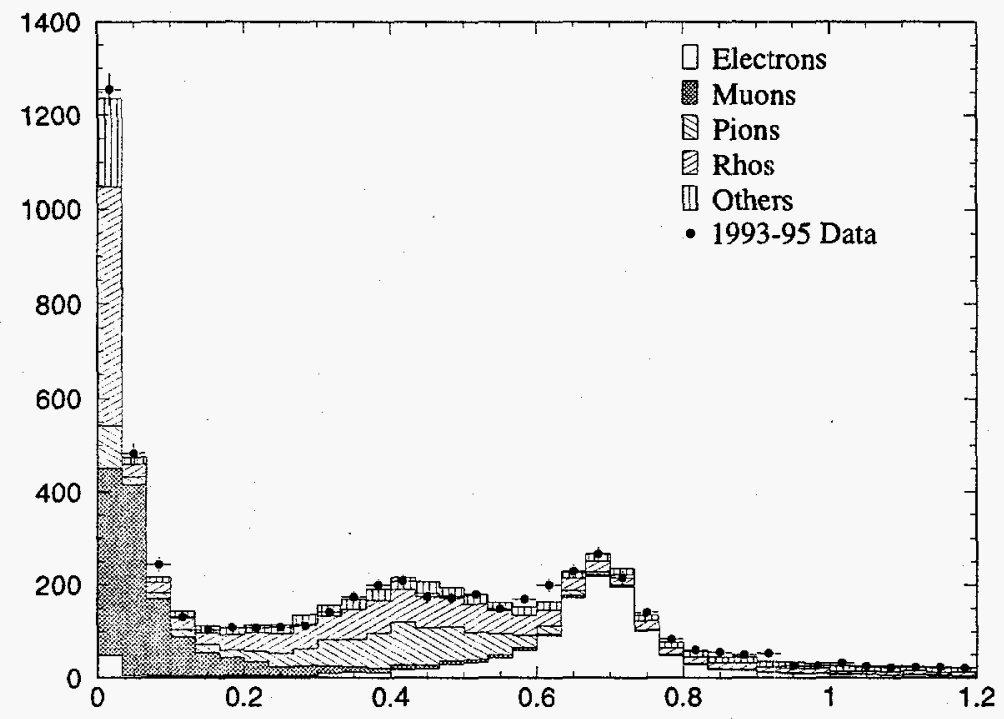

Figure 5.1: E over p. (Min-I E)

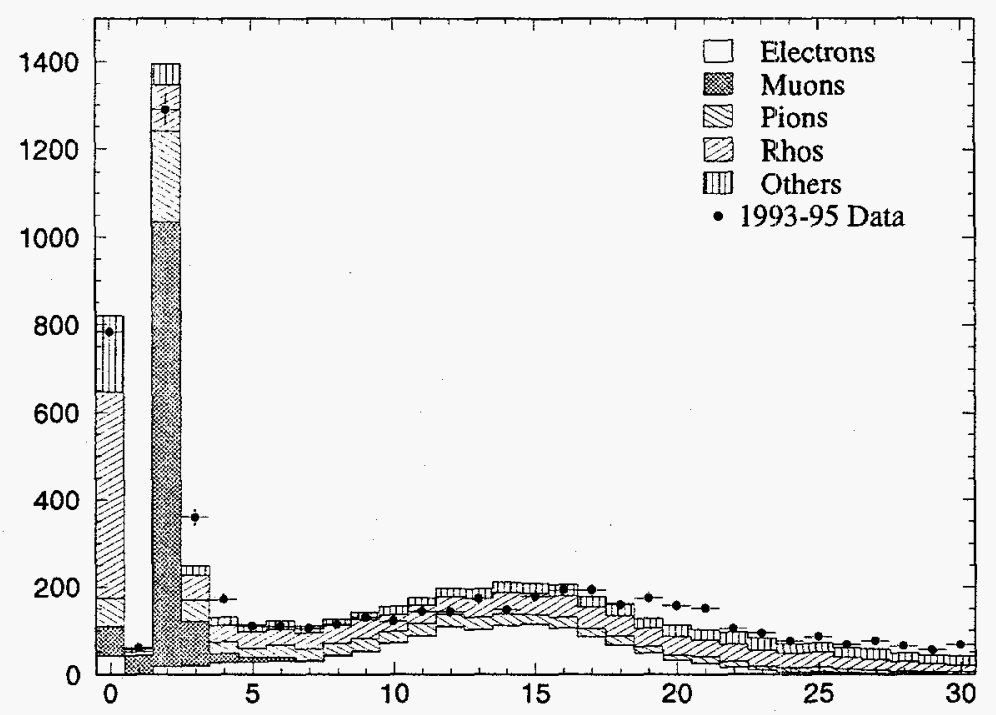

Figure 5.2: Number of EM towers. 


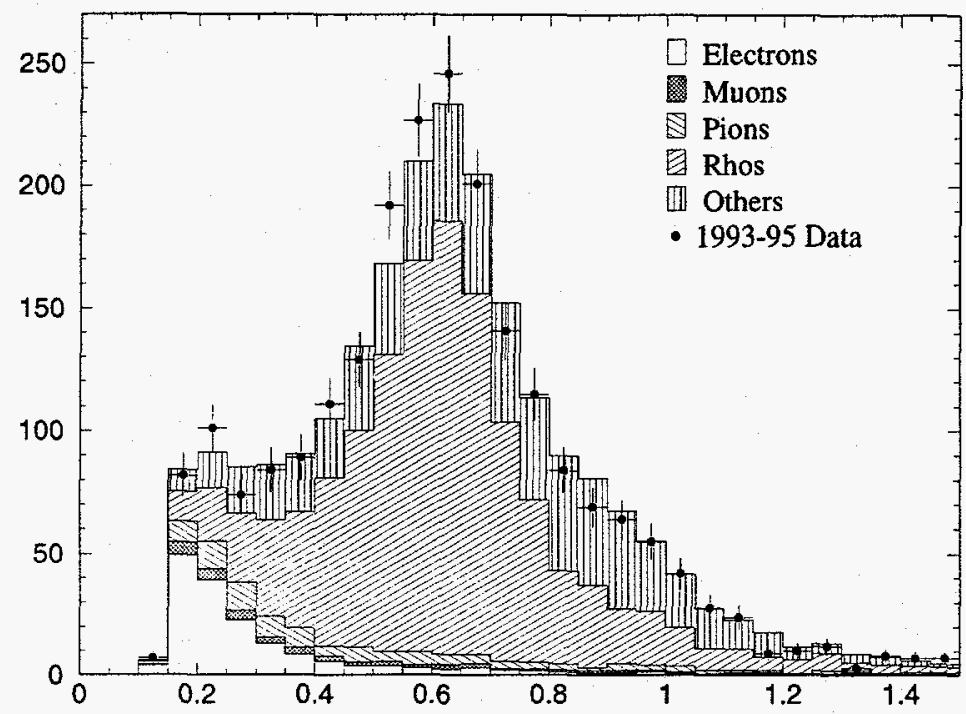

Figure 5.3: Jet mass. (GeV)

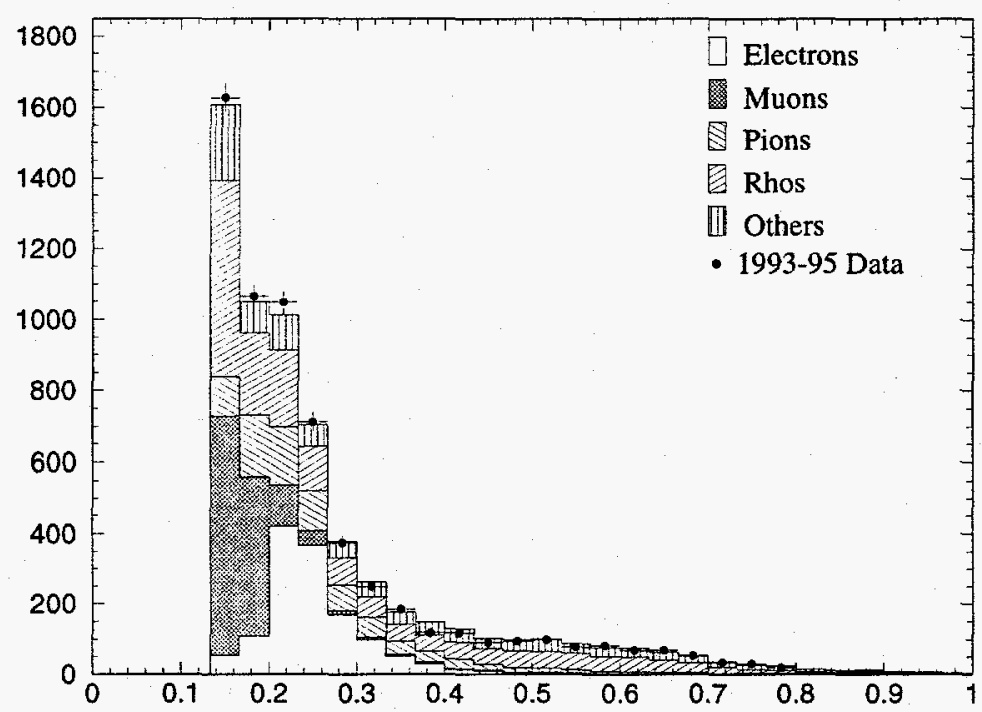

Figure 5.4: Pseudo mass. (GeV) 


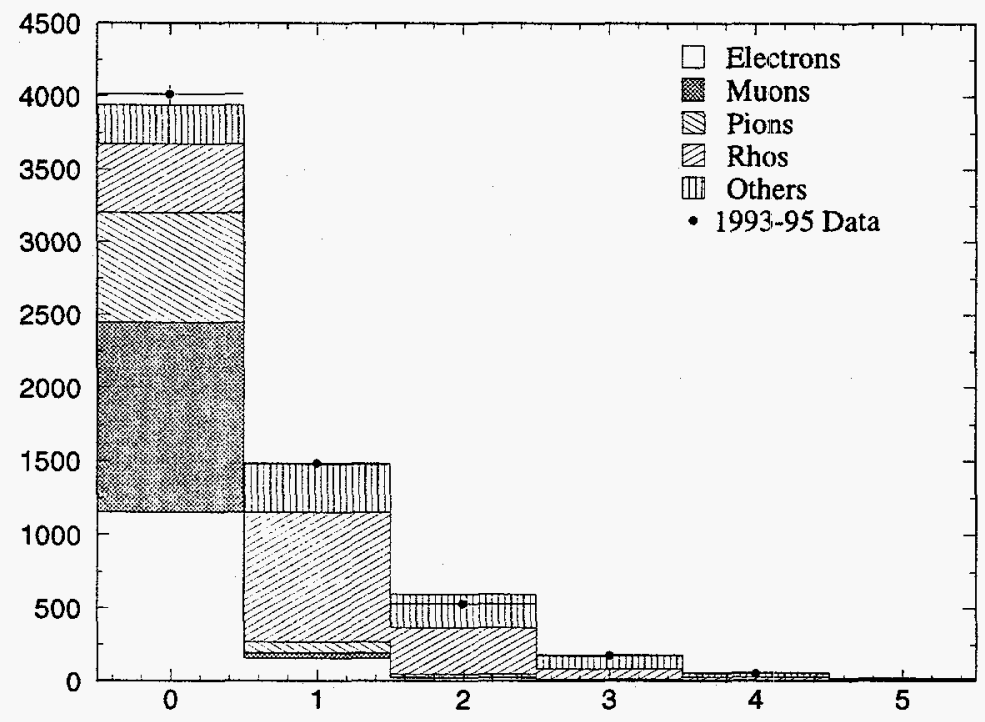

Figure 5.5: Number of neutrals.

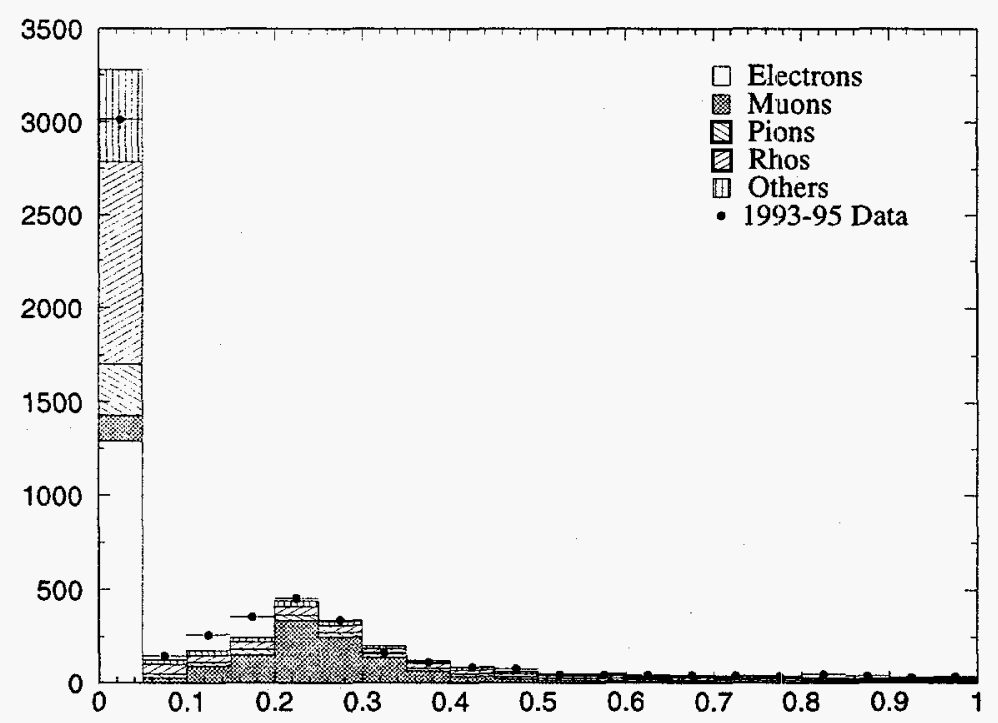

Figure 5.6: HAD2 energy. (Min-I GeV) 


\subsection{Efficiencies and Backgrounds}

Using the Monte Carlo, we can determine the efficiency of the leptonic event selection and the level of backgrounds in the selected samples. This process is analogous to the method we used to determine the overall tau selection efficiency. Because we limit the angular region for particle identification, we find that the identification efficiencies are quite uniform between run periods and are independent of weather the energy trigger is read out or the level of SLC muon background, the primary causes of variations of the tau selection efficiency between running periods. This greatly simplifies our task. In the case of backgrounds, we will have backgrounds from two sources, mis-identified taus and non-tau events. We believe that the Monte Carlo simulation is good enough to give reasonable estimates for the efficiencies and backgrounds. The quantities that are not well modeled are peripheral to the selections, which are essentially based on E/p or WIC hits, with the other cuts used to clean up the resulting samples. As a result, these cuts are loose, and small variations in the cut quantity should have little effect on the overall efficiency or level of background. As a cross check, we check to see if the number of selected leptons in the data agree well with the expected numbers from the well measured leptonic branching ratios of the tau.

\subsubsection{Electron Selection Efficiency and Background}

From the Monte Carlo, we find that the overall electron selection efficiency is 0.38 with an estimated background fraction of $3.7 \%$ roughly evenly divided between mis-identified tau decays and tracks from $\mathrm{WAB}$ and two photon background events. If we confine ourselves to events that pass the tau filter, the efficiency becomes 0.63 . 
Table 5.3: Composition of MC Electronic Decay Sample.

\begin{tabular}{l|r|r|r}
\hline \multicolumn{1}{c|}{ Type } & Raw \# & Scaled \# & Frac. \\
\hline $\mathrm{Z} \rightarrow \tau \rightarrow \mathrm{e}$ & 18289 & 18289 & 0.963 \\
\hline $\mathrm{Z} \rightarrow \tau \rightarrow \pi$ & 159 & 159 & 0.008 \\
\hline $\mathrm{Z} \rightarrow \tau \rightarrow \rho$ & 158 & 158 & 0.008 \\
\hline $\mathrm{Z} \rightarrow \tau \rightarrow$ other $^{\mathrm{a}}$ & 83 & 83 & 0.004 \\
\hline $\mathrm{WAB}$ & 14 & 155 & 0.008 \\
\hline $2 \gamma$ & $15+4^{\mathrm{b}}$ & 152 & 0.008 \\
\hline Total BG & & 707 & 0.037 \\
\hline
\end{tabular}

a. primarily $\mathrm{a}_{1}$

b. 15 two photon to electrons and 4 two photon to taus

As a cross check, we can estimate the tau electronic branching ratio from the electron selection efficiency and background fraction and the tau selection efficiency and background fraction.

$$
\mathrm{BR}(\tau \rightarrow \mathrm{e}) \sim \frac{\frac{\mathrm{N}_{\text {sel e }} \cdot \pi_{\mathrm{e}}}{\varepsilon_{\mathrm{e}}}}{\frac{2 \cdot \mathrm{N}_{\text {sel } \tau} \cdot \pi_{\tau}}{\varepsilon_{\tau}}}=\frac{\mathrm{N}_{\mathrm{e}}}{\mathrm{N}_{\tau}}
$$

We get

Table 5.4: Tau Electronic Branching Ratio Estimate.

\begin{tabular}{l|r|r|r|r|r|r|r|r|c}
\hline Run & $\mathrm{N}_{\text {sel } \tau}$ & $\varepsilon_{\tau}$ & $\pi_{\tau}$ & $\mathrm{N}_{\tau}$ & $\mathrm{N}_{\text {sel } \mathrm{e}}$ & $\varepsilon_{\mathrm{e}}$ & $\pi_{\mathrm{e}}$ & $\mathrm{N}_{\mathrm{e}}$ & $\mathrm{BR}$ \\
\hline 93 & 1308 & 0.53 & 0.989 & 4882 & 326 & 0.38 & 0.963 & 826 & 0.17 \\
\hline $94 \mathrm{~s}$ & 548 & 0.51 & 0.989 & 2125 & 121 & 0.38 & 0.963 & 307 & 0.14 \\
\hline $94 \mathrm{f}$ & 1510 & 0.60 & 0.989 & 4978 & 302 & 0.38 & 0.963 & 765 & 0.15 \\
\hline 95 & 962 & 0.61 & 0.989 & 3119 & 214 & 0.38 & 0.963 & 542 & 0.17 \\
\hline Total & & & & 15104 & & & & 2440 & 0.16 \\
\hline
\end{tabular}


The World Average BR $(\tau \rightarrow \mathrm{e})=0.1778 \pm 0.0007,[7]$ but the statistical uncertainty of the estimate is of order 0.01 , so we are well within the region of a statistical fluctuation. In any case, it does not seem that we are drastically underestimating the background contamination.

\subsubsection{Muon Selection Efficiency and Background}

From the Monte Carlo, we find that the overall muon selection efficiency is 0.43 with an estimated background fraction of $5.9 \%$ roughly evenly divided between mis-identified tau decays and tracks from mu-pair and two photon background events. If we confine ourselves to events that pass the tau filter, the efficiency becomes 0.73 .

Table 5.5: Composition of MC Muonic Decay Sample.

\begin{tabular}{l|r|r|r}
\hline \multicolumn{1}{c|}{ Type } & Raw \# & Scaled \# & Frac. \\
\hline$Z \rightarrow \tau \rightarrow \mu$ & 20378 & 20378 & 0.941 \\
\hline$Z \rightarrow \tau \rightarrow \pi$ & 253 & 253 & 0.012 \\
\hline$Z \rightarrow \tau \rightarrow \rho$ & 229 & 229 & 0.011 \\
\hline$Z \rightarrow \tau \rightarrow$ other $^{\text {a }}$ & 190 & 190 & 0.009 \\
\hline mu-pair & 65 & 430 & 0.020 \\
\hline $2 \gamma$ & $19+3^{\mathrm{b}}$ & 168 & 0.008 \\
\hline Total BG & & 1270 & 0.059 \\
\hline
\end{tabular}

a. primarily $a_{1}$

b. 19 two photon to muons and 3 two photon to taus.

As in the electron case, we can estimate the tau muonic branching ratio as a cross check to the efficiencies and purities. 
Table 5.6: Tau Muonic Branching Ratio Estimate.

\begin{tabular}{l|r|r|r|r|r|c|c|c|c}
\hline Run & $\mathrm{N}_{\text {sel } \tau}$ & $\varepsilon_{\tau}$ & $\pi_{\tau}$ & $\mathrm{N}_{\tau}$ & $\mathrm{N}_{\text {sel } \mu}$ & $\varepsilon_{\mu}$ & $\pi_{\mu}$ & $\mathrm{N}_{\mu}$ & $\mathrm{BR}$ \\
\hline 93 & 1308 & 0.53 & 0.989 & 4882 & 364 & 0.43 & 0.941 & 797 & 0.16 \\
\hline $94 \mathrm{~s}$ & 548 & 0.51 & 0.989 & 2125 & 143 & 0.43 & 0.941 & 313 & 0.15 \\
\hline $94 \mathrm{f}$ & 1510 & 0.60 & 0.989 & 4978 & 408 & 0.43 & 0.941 & 893 & 0.18 \\
\hline 95 & 962 & 0.61 & 0.989 & 3119 & 257 & 0.43 & 0.941 & 562 & 0.18 \\
\hline Total & & & & 15104 & & & & 2565 & 0.17 \\
\hline
\end{tabular}

The World Average $\mathrm{BR}(\tau \rightarrow \mu)=0.1730 \pm 0.0008$.[7] So it seems that we are doing a good job estimating the muon identification efficiency and background. 


\section{Chapter 6}

\section{Fit Technique}

Having selected samples of leptonic tau decays, we must now extract the Michel parameters. We do this by an unbinned maximum likelihood fit of the theoretical tau production and decay spectrum to the data, where we allow the Michel parameters to vary, and where the theoretical spectrum is corrected for radiation, detector effects and background. These corrections are determined from Monte Carlo studies.

\subsection{Fit function}

Eq. 1.26 defines the combined tau production and decay cross section for leptonic decays proportional to a theoretical function $\mathrm{W}\left(\mathrm{x}, \cos \theta, \mathrm{P}_{\mathrm{e}}\right)$. This does not exactly describe the data. The measured values of $x$ and $\cos \theta$ will be smeared by detector resolution effects. Also, the data include background events that we don't expect to follow the theoretical distribution. In addition, the theoretical calculations neglected the final state lepton masses, initial and final state radiation, and were limited to the tree level diagrams for tau production and decay, ignoring higher order processes and the presence of the photon. As a result, we must correct the theoretical spectrum in order to construct a likelihood function to fit the data. The likelihood function must then be properly normalized as a function of the Michel parameters to allow a correct fit. To further complicate matters, the corrections are different for the two decays.

\subsubsection{Corrected Theoretical Spectrum}

We can write the corrected theoretical spectrum for the tau decay to a lepton as 


$$
\begin{aligned}
& W(x, c, P)=B_{\text {non } \tau}(x, c, P)+B_{\tau}(x, c, P)+ \\
& \varepsilon_{\text {sel }}(x, c, P) \cdot \int_{0}^{1} \int_{-1}^{1} R\left(x, x^{\prime}, c, c^{\prime}, P\right) \cdot \varepsilon_{t r k}\left(x^{\prime}, c^{\prime}, P\right) \cdot C\left(x^{\prime}, c^{\prime}, P\right) \cdot W\left(x^{\prime}, c^{\prime}, P\right) d c^{\prime} d x^{\prime}
\end{aligned}
$$

where $B_{\text {non } \tau}$ and $B_{\tau}$ are non-tau and tau background respectively, $\varepsilon_{\text {sel }}$ is the selection efficiency, $\mathrm{R}$ is the resolution function, $\varepsilon_{\text {trk }}$ is the tracking efficiency, $\mathrm{C}$ is the radiative correction, and $\mathrm{W}$ is the uncorrected theoretical spectrum. Here $\mathrm{x}, \mathrm{c}$ and $\mathrm{P}$ are the measured scaled energy $\left(\mathrm{p} / \mathrm{E}_{\mathrm{beam}}\right), \cos \theta$ and beam polarization and $\mathrm{x}^{\prime}$ and $\mathrm{c}^{\prime}$ are the theoretical or true scaled energy and $\cos \theta$. Because the beam polarization is well measured with a relatively small spread and the dependency of the spectrum on polarization is relatively linear, we will take the uncertainty on the beam polarization as a systematic effect, rather than trying to come up with a polarization resolution function. All of the correction functions are in principal different for the two leptonic tau decays. When we evaluate these corrections with the Monte Carlo simulations, we find that in many cases, they are not as complicated as the general expression would suggest.

Because the evaluation of a double integral in equation 6.1 can be complicated and slow, we would like to take advantage of the fact that W can be written as the sum of products of functions of momentum and functions of angle and polarization:

$$
\begin{aligned}
& \mathrm{W}(\mathrm{x}, \mathrm{c}, \mathrm{P})=\frac{1}{\sigma} \frac{\mathrm{d} \sigma}{\mathrm{dc}}(\mathrm{P}, \mathrm{c})\left[\mathrm{f}(\mathrm{x})+\mathrm{P}_{\tau}(\mathrm{P}, \mathrm{c}) \mathrm{g}(\mathrm{x})\right], \\
& =\frac{1}{\sigma} \frac{\mathrm{d} \sigma}{\mathrm{dc}}(\mathrm{P}, \mathrm{c}) \mathrm{f}(\mathrm{x})+\frac{1}{\sigma} \frac{\mathrm{d} \sigma}{\mathrm{dc}}(\mathrm{P}, \mathrm{c}) \mathrm{P}_{\tau}(\mathrm{P}, \mathrm{c}) \mathrm{g}(\mathrm{x})
\end{aligned}
$$

and factor the double integral over $\mathrm{x}^{\prime}$ and $\mathrm{c}^{\prime}$ into a product of separate $\mathrm{x}^{\prime}$ and $\mathrm{c}^{\prime}$ integrals. This requires that we be able to factor the corrections into products of momentum dependant parts and angle and polarization dependent parts. Fortunately, we find that we can 
write the corrections as a product of a scaled momentum term which is only weakly dependant on angle and a angular term which is only weakly dependant on scaled momentum. In this case, we can use the measured values of scaled momentum or angle for the weak dependence, as these reflect the theoretical values quite well. This allows us to factor the integral with little loss of accuracy.

Since the tracking efficiency and resolution functions correct for detector effects that are due to the individual track parameters, we expect them to be independent of the beam polarization, which has no bearing on the tracking of individual charged particles. We also find that the tracking efficiency is independent of scaled momentum in the kinematic region of the fit $(0.035<x<1)$ and thus can be written as a function of angle alone. The resolutions are more complicated in that they are dependant on both momentum and angle. Fortunately, the resolution is good enough that we can use the measured scaled momentum or angle as a first approximation for the true value when calculating the resolution for the opposite quantity and only make a small error in the calculation of resolution effects.

For the radiative corrections, the angular corrections are absorbed into the corrected $A_{\ell}$ value, leaving only corrections to the momentum spectrum which vary with angle and polarization only due to the differing scaled momentum spectra for left and right-handed tau decays. For the selection efficiency and tau background corrections, we also find that part of the angular and polarization dependence comes from the differing scaled momentum-spectra for left and right-handed taus. As a result, we write these correction functions as combinations of correction functions for left-handed taus and for right-handed taus, weighted by the expected theoretical fraction of left or right-handed taus for the given angle and beam polarization. We can write a correction $F$ as:

$$
F=n_{L}(P, c) \cdot F_{L}+n_{R}(P, c) \cdot F_{R}
$$


where

$$
\mathrm{n}_{\mathrm{L}(\mathrm{R})}(\mathrm{P}, \mathrm{c})=\frac{\frac{\mathrm{d} \sigma_{\mathrm{L}(\mathrm{R})}}{\mathrm{dc}}(\mathrm{P}, \mathrm{c})}{\frac{\mathrm{d} \sigma}{\mathrm{dc}}(\mathrm{P}, \mathrm{c})}
$$

The remaining angular dependence of the tau background is identical to that of the taupair production cross section.

The corrections for left and right-handed taus can be determined from the Monte Carlo where we can separate the left and right-handed tau decays. For the radiative correction and the selection efficiency, this assumes that we are measuring quantities near the Standard Model expectations, as these corrections depend on the parameters we are measuring, and the Monte Carlos are generated using Standard Model parameters. Fortunately, even large variations of the Michel parameters make only a relatively small change in the actual scaled momentum spectra we expect to measure, so the choice of this parameterization should not have a big effect on the result. For the tau background, this only assumes that non-leptonic tau decays proceed as the Standard Model predicts.

We find that the non-tau background follows an isotropic angular distribution modulated by the left-right cross section asymmetry due to the $\mathrm{Z}^{0}$ boson parity violation and by a function of scaled momentum:

$$
\mathrm{B}_{\text {non } \tau}(\mathrm{x}, \mathrm{c}, \mathrm{P})=\mathrm{B}_{\text {non } \tau}(\mathrm{x}) \cdot \frac{3}{16}\left(1-\mathrm{P} \cdot \mathrm{A}_{\mathrm{e}}\right)\left(1-\cos ^{2} \theta\right)
$$

Actually, the MC background events that pass the tau filter are also statistically consistent with equal right and left-handed cross sections, but knowing that a good portion of the background is due to $Z^{0}$ events, we choose to use the asymmetric cross section, although the two photon contribution should be nearly symmetric. 
We can now rewrite the corrected theoretical spectrum as

$$
\begin{aligned}
& W^{\prime}(x, c, P)=B_{\text {non } \tau}(x) \cdot \frac{3}{16}\left(1-P \cdot A_{e}\right)\left(1-c^{2}\right)+ \\
& \left(n_{L}(P, c) \cdot B_{\tau_{L}}(x)+n_{R}(P, c) \cdot B_{\tau_{R}}(x)\right) \cdot \frac{1}{\sigma} \frac{d \sigma}{d c}(P, c)+ \\
& \left(\mathrm{n}_{\mathrm{L}}(\mathrm{P}, \mathrm{c}) \cdot \varepsilon_{\mathrm{sel \times}}(\mathrm{x}) \cdot \varepsilon_{\mathrm{selc}}(\mathrm{c})+\mathrm{n}_{\mathrm{R}}(\mathrm{P}, \mathrm{c}) \cdot \varepsilon_{\mathrm{sel \times} R}(\mathrm{x}) \cdot \varepsilon_{\mathrm{selcR}}(\mathrm{c})\right) \text {. } \\
& {\left[\left(\int_{-1}^{1} R_{c}\left(x, c, c^{\prime}\right) \cdot \varepsilon_{t r k}\left(c^{\prime}\right) \cdot \frac{1}{\sigma} \frac{d \sigma}{d c}\left(P, c^{\prime}\right) d c^{\prime}\right.\right. \text {. }} \\
& \left.\int_{0}^{1} R_{x}\left(x, x^{\prime}, c\right) \cdot\left(n_{L}(P, c) \cdot C_{L}\left(x^{\prime}\right)+n_{R}(P, c) \cdot C_{R}\left(x^{\prime}\right)\right) \cdot f\left(x^{\prime}\right) d x^{\prime}\right)+ \\
& \left(\int_{-1}^{1} R_{c}\left(x, c, c^{\prime}\right) \cdot \varepsilon_{t r k}\left(c^{\prime}\right) \cdot \frac{1}{\sigma} \frac{d \sigma}{d c}\left(P, c^{\prime}\right) \cdot P_{\tau}\left(P, c^{\prime}\right) d c^{\prime} \cdot\right. \\
& \left.\left.\int_{0}^{1} R_{x}\left(x, x^{\prime}, c\right) \cdot\left(n_{L}(P, c) \cdot C_{L}\left(x^{\prime}\right)+n_{R}(P, c) \cdot C_{R}\left(x^{\prime}\right)\right) \cdot g\left(x^{\prime}\right) d x^{\prime}\right)\right]
\end{aligned}
$$

which appears more complicated than the previous expression, but is actually quite a bit simpler to evaluate as we have reduced most of the correction functions other than the resolution to one dimensional functions and factored the double integral. All of the correction functions are still, in principal, different for the two decay channels. Unfortunately, the only correction that is common to the two decays is the $\cos \theta$ resolution, which is probably what we would expect since most of the other corrections are dependent on the decay identification.

\subsubsection{The Likelihood Function}

Now that we have a corrected theoretical spectrum, we can construct the likelihood function. The likelihood is defined as the product of the probabilities for each selected event given the values of the parameters we wish to fit. Unfortunately, because the normalization of $\mathrm{W}^{*}$ depends on the Michel parameters, $\mathrm{W}^{\prime}$ is not a probability distribution function. As a result, we must normalize $\mathrm{W}^{\prime}$ so that it integrates and sums to one over the range of 
all the variables we measure for the fit for a given set of Michel parameters. Having done this, we can write the likelihood function

$$
\mathcal{L}(\rho, \xi,(\xi \delta), \eta)=\prod_{i} \frac{W^{\prime}\left(x_{i}, c_{i}, P_{i} ; \rho, \xi,(\xi \delta), \eta\right)}{\sum_{P_{e}} \iint W^{\prime}\left(x, c, P_{e} ; \rho, \xi,(\xi \delta), \eta\right) d x d c}
$$

where the sum over polarization states is for positive and negative electron beam polarization, and the integrals range over the kinematic region of the fit, and the product runs over all decays of the specified type. The Michel parameters $\rho, \xi,(\xi \delta)$, and $\eta$ enter into $W^{\prime}$ through the $f$ and $g$ functions. Fortunately, $\mathrm{W}^{\prime}$ is linear in the Michel parameters, so we need only evaluate the integral once and can recalculate the normalization for different values of the Michel parameters

$$
\begin{aligned}
& N(\rho, \xi,(\xi \delta), \eta)=\sum_{P_{e}} \iint W^{\prime}\left(x, c, P_{e} ; \rho, \xi,(\xi \delta), \eta\right) d x d c= \\
& \sum_{\mathrm{P}_{\mathrm{e}}} \iint\left(\mathrm{B}_{\text {non } \tau}+\mathrm{B}_{\tau}+\varepsilon_{\text {sel }} \cdot \iint \mathrm{R} \cdot \varepsilon_{\mathrm{trk}} \cdot \mathrm{C} \cdot \mathrm{Wdc^{ \prime } \mathrm { dx }}\right) \mathrm{dxdc}= \\
& \sum_{P_{e}} \iint\left(B_{\text {non } \tau}+B_{\tau}+\varepsilon_{\text {sel }} \cdot \iint R \cdot \varepsilon_{\text {trk }} \cdot C \cdot \frac{1}{\sigma} \frac{d \sigma_{c}}{d c} c_{c}\left(x^{\prime}\right) d c^{\prime} d x^{\prime}\right) d x d c+ \\
& \rho \sum_{P_{e}} \iint\left(\varepsilon_{\text {sel }} \cdot \iint R \cdot \varepsilon_{\text {trk }} \cdot C \cdot \frac{1}{\sigma} \frac{d \sigma}{d c} f_{\rho}\left(x^{\prime}\right) d c^{\prime} d x^{\prime}\right) d x d c+ \\
& \eta \sum_{\mathrm{P}_{\mathrm{e}}} \iint\left(\varepsilon_{\mathrm{sel}} \cdot \iint \mathrm{R} \cdot \varepsilon_{\mathrm{trk}} \cdot \mathrm{C} \cdot \frac{1}{\sigma} \frac{\mathrm{d} \sigma_{\mathrm{f}}}{\mathrm{dc}} \mathrm{f}_{\eta}\left(\mathrm{x}^{\prime}\right) \mathrm{dc^{ \prime }} \mathrm{dx} \mathrm{x}^{\prime}\right) \mathrm{dxdc}+ \\
& \xi \sum_{\mathrm{P}_{\mathrm{e}}} \iint\left(\varepsilon_{\mathrm{sel}} \cdot \iint \mathrm{R} \cdot \varepsilon_{\mathrm{trk}} \cdot \mathrm{C} \cdot \frac{1}{\sigma} \frac{\mathrm{d} \sigma}{\mathrm{dc}} \cdot \mathrm{P}_{\tau} \mathrm{g}_{\xi}\left(\mathrm{x}^{\prime}\right) \mathrm{dc} \mathrm{c}^{\prime} \mathrm{dx}\right) \mathrm{dxdc}+ \\
& \xi \delta \sum_{\mathrm{P}_{\mathrm{e}}} \iint\left(\varepsilon_{\mathrm{sel}} \cdot \iint \mathrm{R} \cdot \varepsilon_{\mathrm{trk}} \cdot \mathrm{C} \cdot \frac{1}{\sigma} \frac{\mathrm{d} \sigma}{\mathrm{dc}} \cdot \mathrm{P}_{\tau} \mathrm{g}_{\xi \delta}\left(\mathrm{x}^{\prime}\right) \mathrm{dc} \mathrm{c}^{\prime} \mathrm{dx}\right) \mathrm{dxdc}= \\
& \mathrm{N}_{C}+\rho \mathrm{N}_{\rho}+\eta \mathrm{N}_{\eta}+\xi \mathrm{N}_{\xi}+\xi \delta \mathrm{N}_{\xi \delta}
\end{aligned}
$$

So now we have a correctly normalized likelihood function that can be evaluated quickly 
once some normalization integrals have been calculated.

$$
\mathcal{L}(\rho, \xi,(\xi \delta), \eta)=\prod_{i} \frac{W^{\prime}\left(x_{i}, c_{i}, P_{i} ; \rho, \xi,(\xi \delta), \eta\right)}{N(\rho, \xi,(\xi \delta), \eta)}
$$

To extract the Michel parameters, we vary them until we find a maximum of the likelihood function. For this purpose, it is easier to work with the log of the likelihood, which replaces the product with a sum

$$
\log \mathcal{L}=\sum_{i} \log \frac{W_{i}^{\prime}}{N}=\sum_{i} \log W_{i}^{\prime}-n_{e v} \log N
$$

In order to combine the result for the two different decays, we need only use the correct probability for each decay. Thus we can write a combined log likelihood

$$
\log \mathcal{L}=\log \left(\prod_{i} \frac{W^{\prime}}{N^{e}} \prod_{j} \frac{W^{\prime \mu}}{N^{\mu}}\right)=\log \mathcal{L}^{e}+\log \mathcal{L}^{\mu}
$$

where $\mathrm{W}^{\mathrm{e}}, \mathrm{N}^{\mathrm{e}}, \mathrm{W}^{\mu}, \mathrm{N}^{\mu}$ are the appropriate functions for each decay, and the products run over all decays of each type.

\subsubsection{Technical Details of Doing the Fit}

Now that we have a likelihood function, we must maximize it in order to perform the fit. We perform the maximization using the CERN library package MINUIT[32], which is designed to perform function minimizations, so we minimize the negative log likelihood. Errors are determined also using MINUIT, with an error contour level set at a function

increase of $\frac{1}{2}$. This gives a one standard deviation error contour for the fit when we are minimizing the negative log likelihood. The integrals in the corrected theoretical spectrum and the normalization are not necessarily analytic, so we choose to evaluate them numerically using an adaptive Gaussian quadrature algorithm.[33] The normalization requires a 
two dimensional version of this algorithm.

\subsection{Corrections}

In order to do the fit, we must determine what the actual correction functions are. To do this, we must first define the measured scaled energy, tau direction, and beam polarization. Then we can use the Monte Carlo samples to evaluate correction functions for these definitions. In all cases, we use the CERN PAW[34] package to do binned $\chi^{2}$ fits to determine a simple functional parameterizations for each correction.

\subsubsection{Defining Quantities}

To do the fit, we must have a beam polarization, a scaled energy and a tau direction (actually only a $\cos \theta$ ) for each identified decay. For the measured beam polarization, we use the magnitude reported by the Compton polarimeter and the sign from the polarized electron source. The Compton polarimeter provides a magnitude of polarization measurement on the order of every ten minutes. Because there are periods where the polarimeter is not running, we require that there be a polarization measurement within 1 hour of each event that we use in the analysis. Also, we require that the magnitude of the polarization be greater than 0.4 , which removes zero polarization events and events where the polarization was being tuned up and most likely was unreliable. Actually, this requirement leaves only those events at the nominal beam polarization for each run, allowing us to assume that there are essentially two beam polarization states for each run, left-handed and righthanded at the measured magnitude of the beam polarization.

We define the measured tau direction as the direction of the difference between the two tau jet directions, where we subtract the direction of the positively charged jet from that of the negatively charged jet. This gives us the negatively charged tau's flight direction. The jet directions come from the iterative procedure described in 4.1.2, and are a momentum 
sum of all tracks and unassociated clusters in a 15 degree cone about the jet direction. Because of the highly boosted nature of the taus and the expectation that they will be produced back to back, this gives us an excellent measure of the tau direction. We then take the cosine of the angle between this direction and the electron beam direction.

The scaled energy is defined differently for electrons and muons. For muons, we take the measured scaled energy to be the momentum from the combined CDC-VXD track fit divided by half of the center of mass energy as measured by the WISRD. Because electrons are much less massive than electrons, they are much more susceptible to bremsstrahlung in flight, particularly in the beam pipe. As a result, we construct a jet momentum out of the CDC-VXD track momentum for the identified track and any unassociated clusters within 15 degrees of the track. In the resolution studies, this greatly reduced the strength of the radiative tail for the electrons. About $10 \%$ of identified electrons have nearby neutrals. In this case, identified photon conversions are treated as clusters.

For the Monte Carlo "truth" values, we use the input beam polarization and the generated beam helicity for each event as the beam polarization. We use the difference between the generated negative tau and positive tau directions to give us the "true" negative tau direction. We do this because the theory is based on the $\mathrm{Z}^{0}$ being produced exactly at rest, thus making the taus exactly back to back. This is not the case in the real world or for the Monte Carlo, and the combination of the two tau flight directions gives us a better approximation of the theoretical quantity. We use the generated decay lepton energy divided by half the generated center of mass energy as the generated scaled energy.

\subsubsection{Radiative Corrections}

These corrections are those that are necessary to go from the theoretical spectrum calculated in Chapter 1 to the actual scaled energy and angular spectrum as calculated by the 
KORALZ Monte Carlo Program. These corrections include the photon terms, initial state radiation, final state radiation, and decay radiation, and final state mass effects. As a result, they are different for electronic and muonic decays. The corrections to the $\cos \theta$ distributions can be incorporated by using the measured value for $\mathrm{A}_{\ell}$, which incorporates all the effects we are correcting for at the $Z^{0}$ resonance. Decay effects are not significant for the $\cos \theta$ distribution.
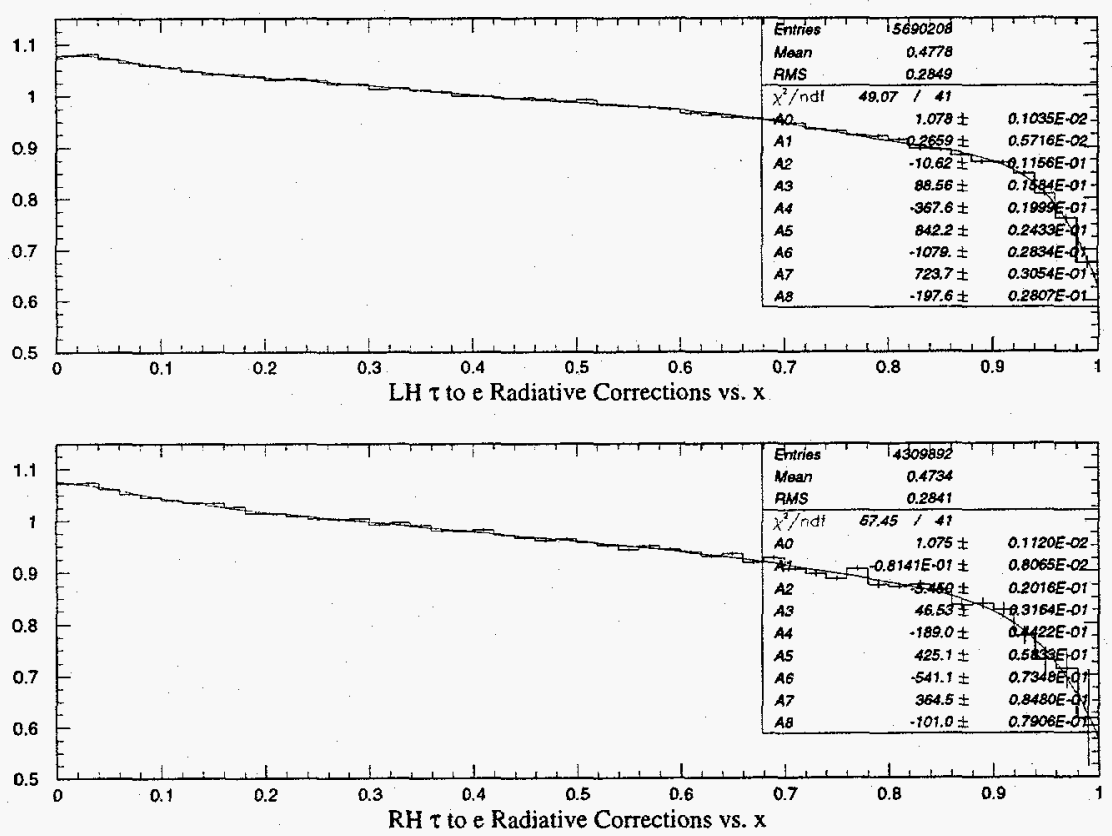

Figure 6.1: Electron radiative corrections vs. scaled energy.

We calculate these corrections by dividing the number of generated events in a given scaled energy bin by the number that the theoretical spectrum predicts for the size Monte Carlo sample generated. Because the two helicity states have different scaled energy spectra, we parameterize this separately for the two tau helicity states. These corrections were created using two samples of 5 million KORALZ tau pairs decaying exclusively to elec- 
trons or to muons. Because KORALZ generates tau pairs and decays them independently of one another, generating uniform decays does not change the result for the radiative corrections.
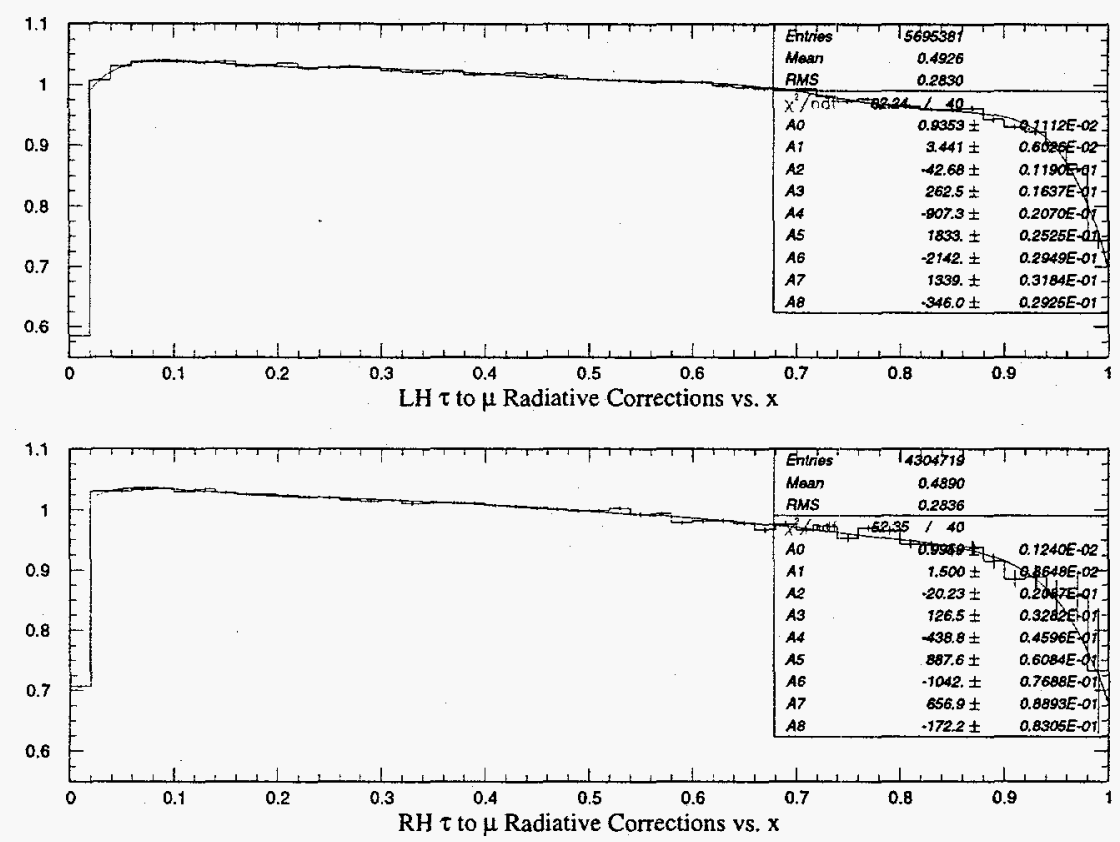

Figure 6.2: Muon radiative corrections vs. scaled energy.

We have calculated the corrections in 50 bins in the "true" scaled energy, and parameterized them by fitting them with eighth order polynomials in the scaled energy. The effects of the differing masses can clearly be seen. The electrons, being lighter have a higher tendency to radiate and thus have more steeply sloping corrections than the muons. The muons, on the other hand have kinematic restrictions on low values for scaled energy due to their higher mass. As a result of this, the muon fit is for the upper 49 points. The muon resolution function at the bottom end of the fit region $(x=0.035)$ is narrow enough to allow us to neglect this lowest bin. We have defined the measured electron energy to 
include nearby neutral clusters, some of these correspond to energy losses that we are correcting for here. As a result, the electron resolution function effectively "backs out" some of this correction.

\subsubsection{Tracking Efficiency}

Because not all charged particles in the SLD register in the tracking systems, we must account for the loss of these tracks. This could be accomplished as a part of the normalization of the resolution function, but since this effect is largely geometric, it is simpler to parameterize it as part of a separate tracking efficiency function. We find that the angular dependence of the tracking efficiency is virtually identical for electrons and muons, but is slightly higher overall for muons because they are less likely to interact with material in the detector before reaching the CDC. The tracking efficiency is independent of "true" scaled energy above the lowest energies, where the narrowness of the resolution functions will minimize the error due to this assumption. Fig. 6.3 show the calculated tracking efficiencies for electrons and muons.

We calculate the tracking efficiency using the full Monte Carlo with detector simulation. We do it in terms of the "true" momenta and angle. A particle is considered tracked if the simulation generates a track that we would consider for the analysis in the hemisphere corresponding to the input particle. This means that the track meets the track quality requirements, has an associated vertex detector hit, is the only track in its hemisphere, and is within the fiducial region. We parameterize this verses angle by fitting it with a three parameter function of the form

$$
\varepsilon_{\text {trk }}(\mathrm{c})=\mathrm{p} 1 \cdot \frac{\mathrm{e}^{\frac{\mathrm{p} 2-|\mathrm{c}|}{\mathrm{p} 3}}}{1+\mathrm{e}^{\frac{\mathrm{p} 2-|\mathrm{c}|}{\mathrm{p} 3}}}
$$


To evaluate this efficiency, we use the full sample of 135092 tau pair Monte Carlo events with full detector simulation and background overlays, representing the 1993 and 1994-5 running periods. This sample includes 48288 electronic tau decays and 46943 muonic tau decays.
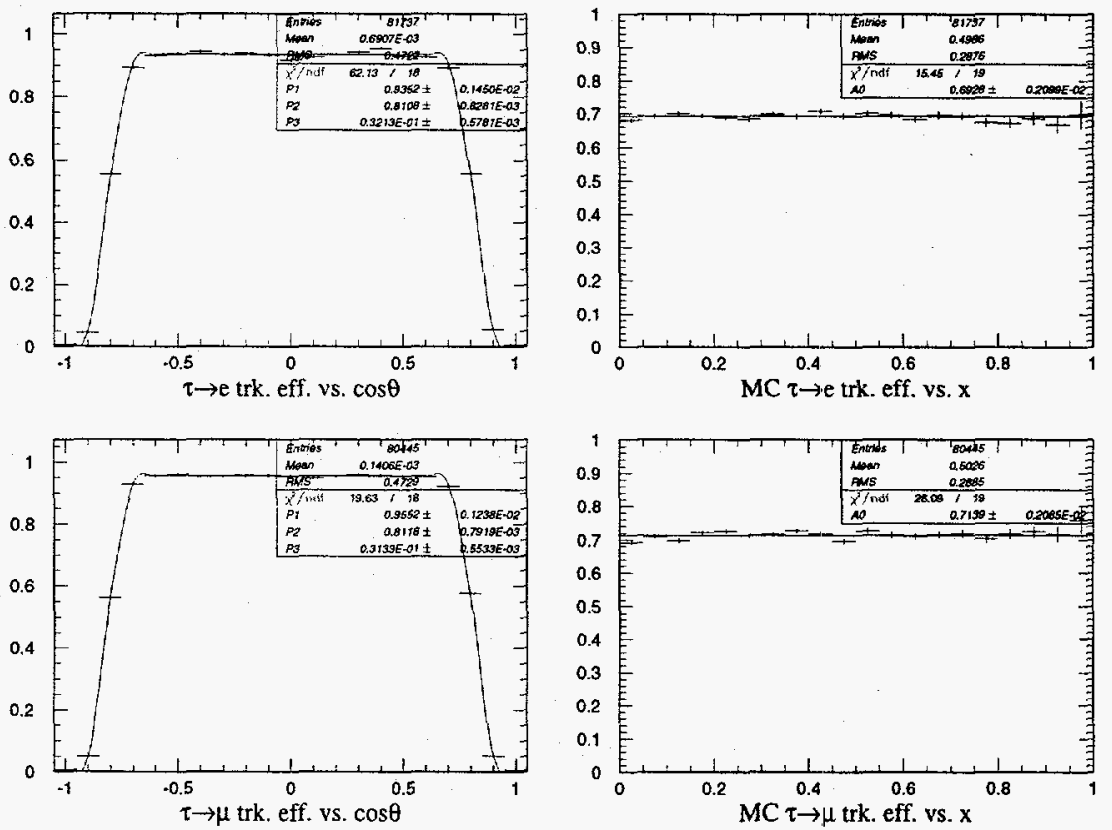

Figure 6.3: Tracking efficiencies.

\subsubsection{Electron and Muon Angular Resolution}

The resolution functions are the most complicated of the correction functions that we require to do the fit. These are potentially functions of three variables. In the case of the angular resolution these variables are the true and measured angle as well as the true scaled momentum. Because we expect the resolution functions to be reasonably narrow, we will take the measured momentum as a good approximation of the true momentum. Because of the nature of the $\cos \theta$ measurement, we will further make the assumption that 
the resolution function represents a distribution of the difference between the absolute values of the measured and true $\cos \theta$ (angular difference), where the width of the distribution is allowed to vary with measured momentum and measured angle. To determine the shape of the angular difference distribution and to determine the dependencies of that distribution on the measured momentum and $\cos \theta$, we plot the angular difference distribution for different bins of measured angle and momentum. We choose one of these bins as a representative shape, and fit all the bins to that shape, determining the dependence of the shape parameters on the momentum and angle.

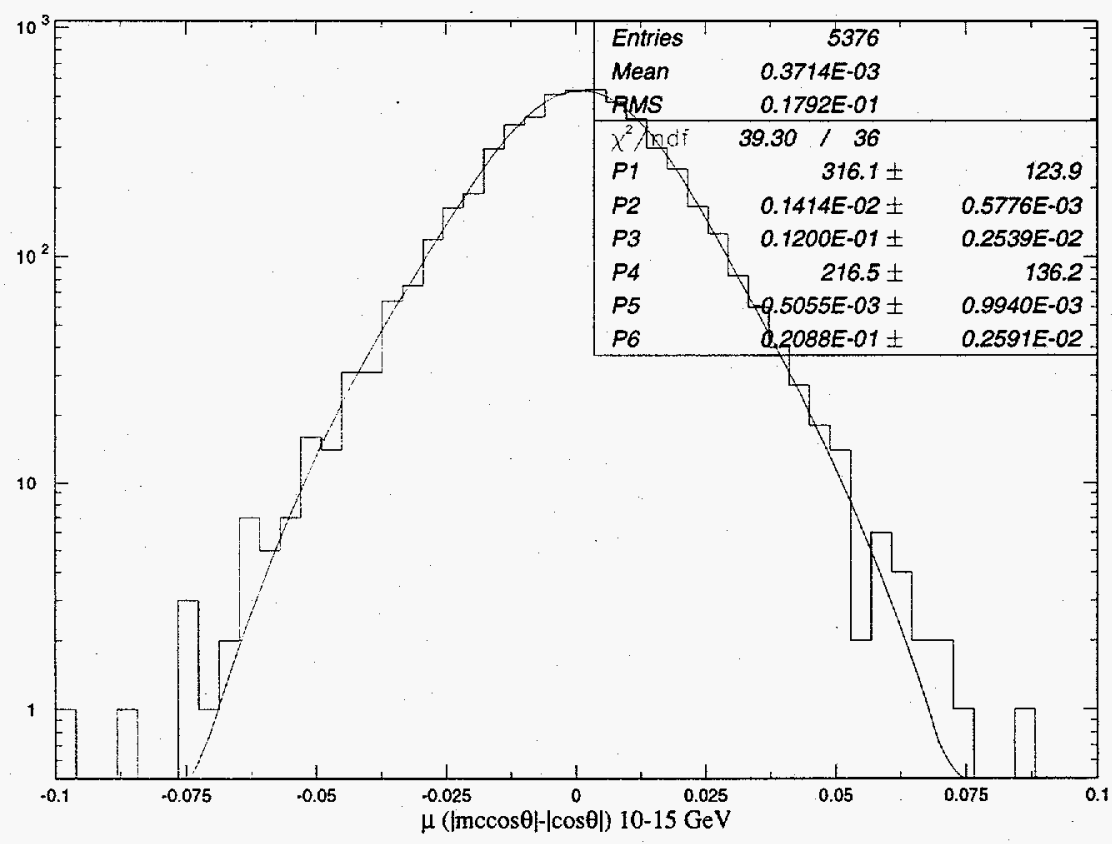

Figure 6.4: Muon $\cos \theta$ resolution shape function.

We determine the shape function for the muon $\cos \theta$ resolution by fitting the angular difference distribution for tracks in the range of 10 to $15 \mathrm{GeV}$ to the sum of two independent Gaussians. The shape function that we define is parameterized by the normalization, width and mean of the narrowest Gaussian in the fit. The second Gaussian is determined by the parameters of the first Gaussian. The width being a multiple of the width of the first 
Gaussian, the normalization being a fraction of the normalization of the first Gaussian, and the mean being offset from the mean of the first Gaussian by some number of widths of the first Gaussian. Thus we get a shape function that looks like

$$
\mathrm{s}(\delta ; \mathrm{N}, \mu, \sigma)=\mathrm{N}\left(\mathrm{e}^{\frac{(\delta-\mu)^{2}}{\sigma^{2}}}+\mathrm{f} \cdot \mathrm{e}^{\frac{(\delta-\mu+\mathrm{g} \sigma)^{2}}{(\mathrm{~h} \sigma)^{2}}}\right)
$$

where $f, g$, and $h$ define the shape, and $\mu$ and $\sigma$ are the parameters of the shape that we must determine by refitting in all bins. $\mathrm{N}$ is an overall normalization, and $\delta$ is the angular difference.

We then use this shape function to fit the angular difference distribution for bins of both momentum and $\cos \theta$ and use the resulting fit parameters to determine their dependence on track momentum and angle by plotting them against the momenta and angles they represent.
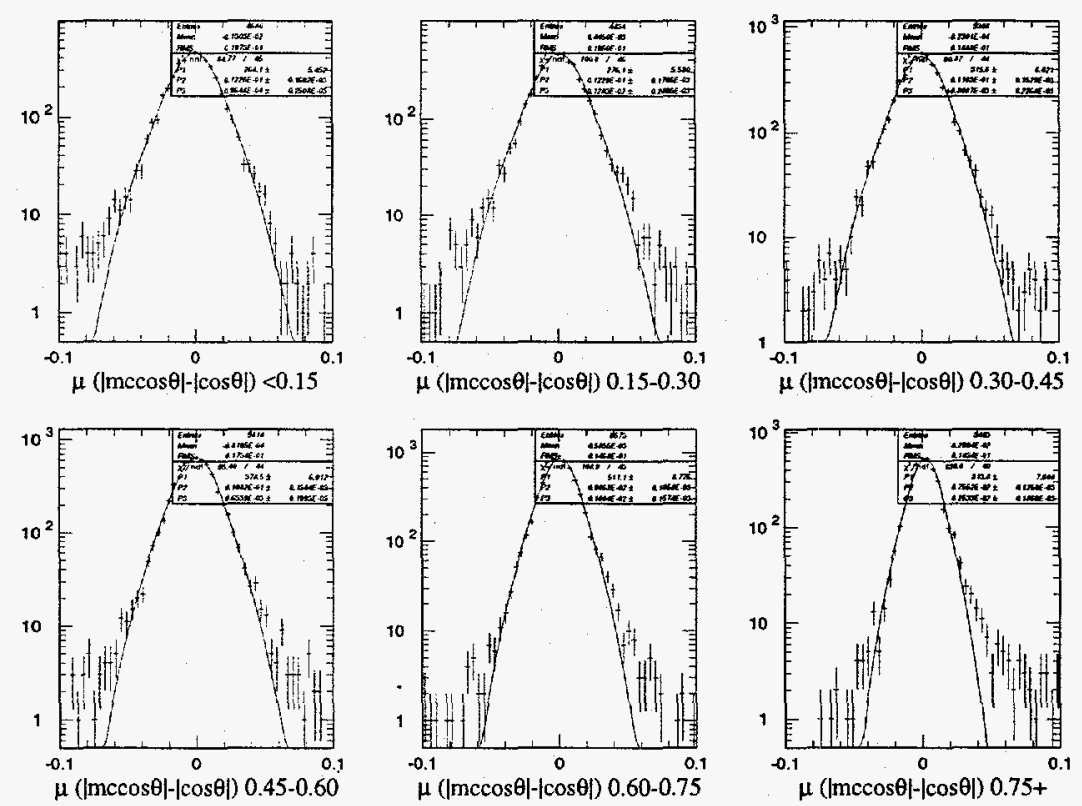

Figure 6.5: Muon $\cos \theta$ resolution fits in $\cos \theta$ bins. 

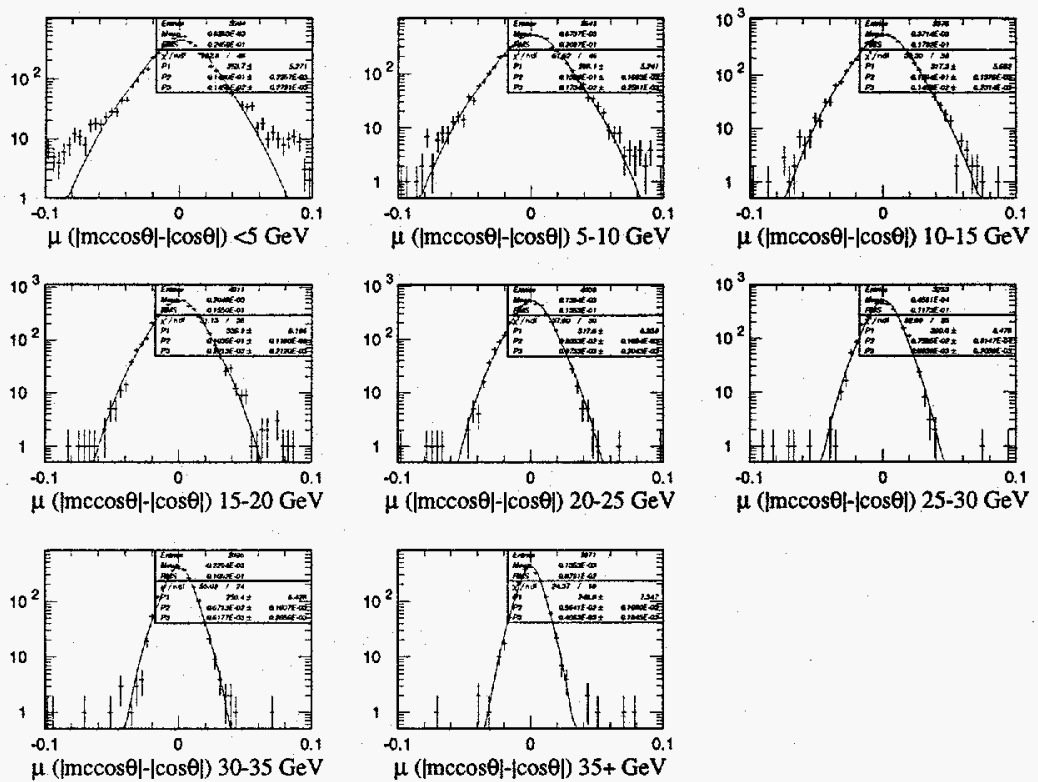

Figure 6.6: Muon $\cos \theta$ resolution fits in momentum bins.
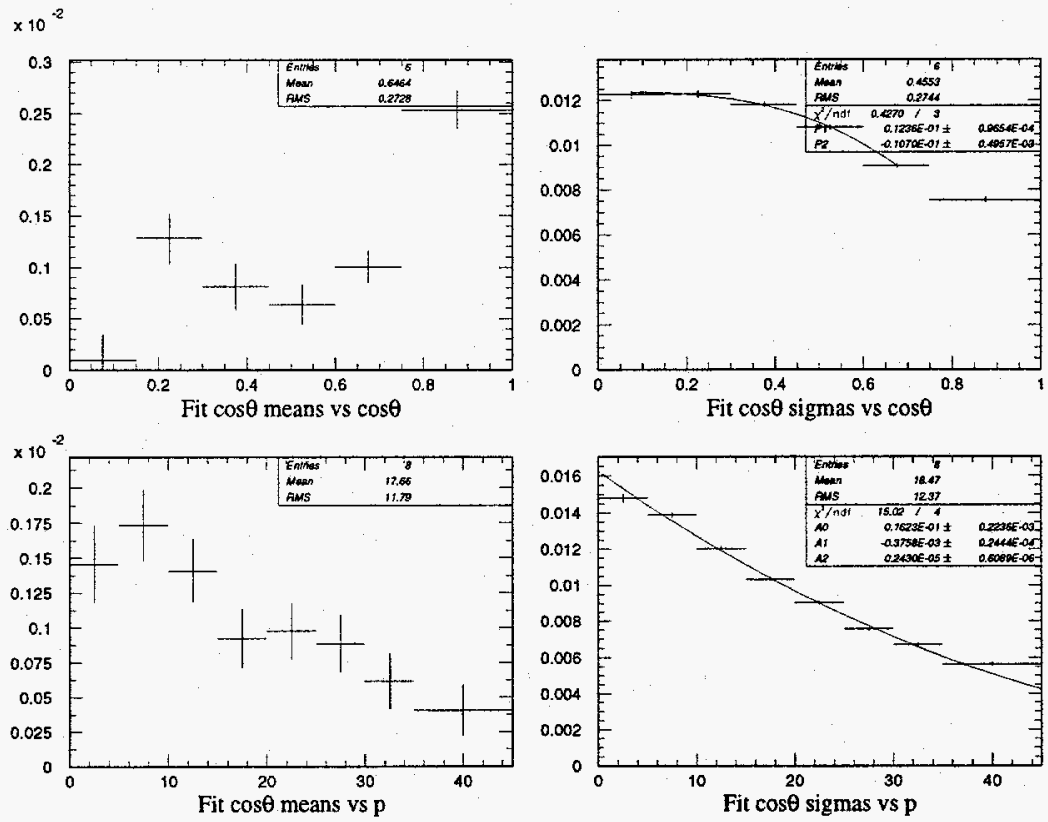

Figure 6.7: Muon $\cos \theta$ resolution fit parameters. 
We find that the mean of the shape function is very weakly dependant on both angle and momentum. We ignore these dependencies and determine the mean from the overall mean of the angular difference distribution. The width of the shape function is significantly dependent on both angle and momentum. We fit the momentum dependence of the width to a second order polynomial and the angular dependence to function of the form $\mathrm{p} 1+\mathrm{p} 2 \mathrm{x}^{3}$. To determine the overall width for a given angle and momentum, we take the product of the two widths divided by the mean width. This gives us all the parameters we need to construct the resolution function.

The resolution function for a given measured and true angle is the probability that a track with the true angle will be measured as the measured angle. Thus if we normalize the shape function to one when integrated over all measured angles for a given true angle and momentum, we will have the appropriate resolution function for those parameters.
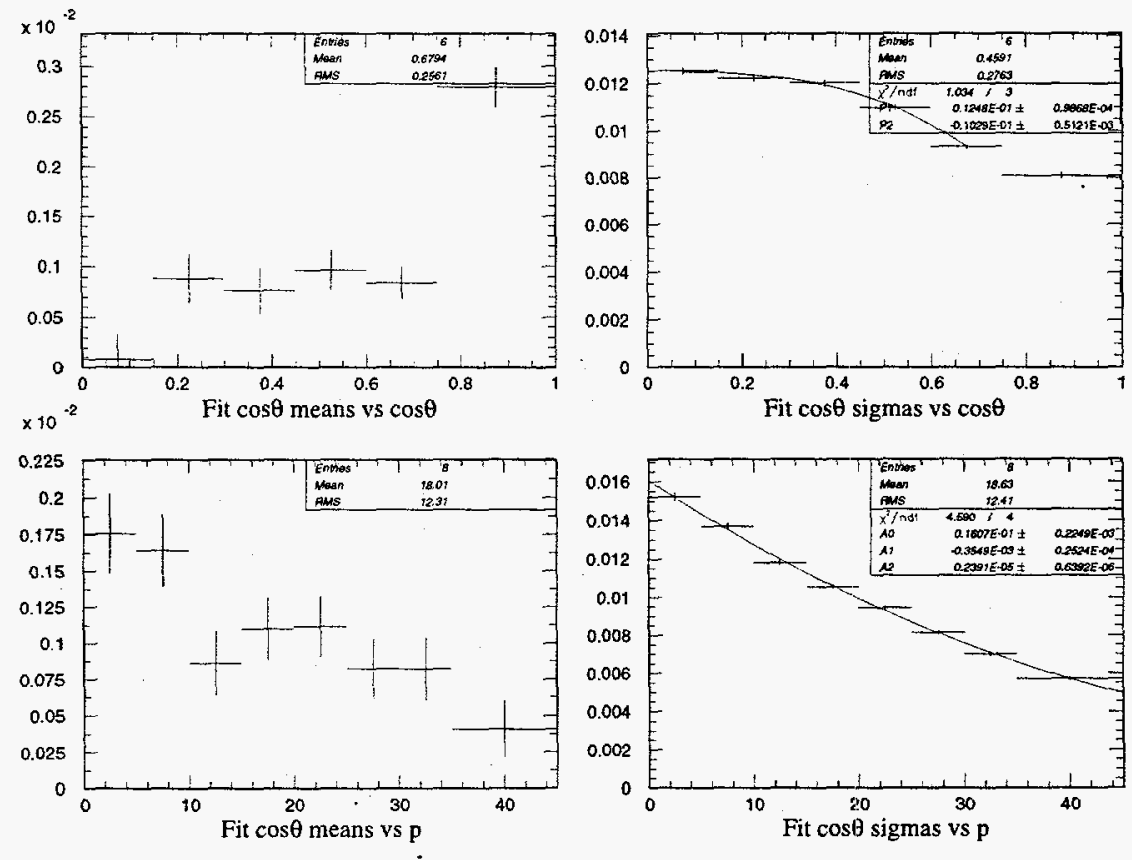

Figure 6.8: Electron $\cos \theta$ resolution fit parameters. 
The electron $\cos \theta$ resolution function is determined in exactly the same way as the muon function. In fact, we find that the shape functions for the two decays are identical, and the resulting parameterizations for the mean and width are only slightly different.

\subsubsection{Muon Momentum Resolution}

We use a procedure virtually identical to the above to determine the muon scaled momentum resolution function. In this case, we expect the function to be a function of the difference of the true and measured momentum divided by the true momentum $(\delta \mathrm{p} / \mathrm{p})$, and to be parameterized by the angle and momentum of the track. As before, we determine a shape function by picking a representative region of the allowed kinematics and plotting the distribution of $\delta \mathrm{p} / \mathrm{p}$.

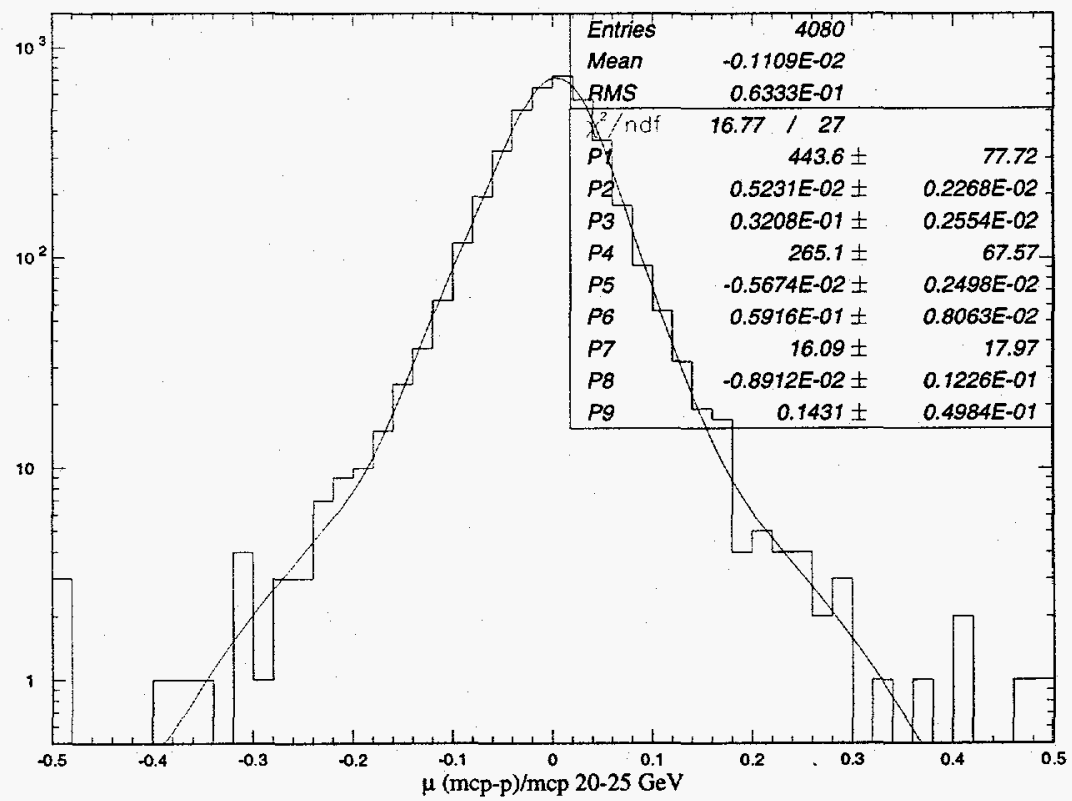

Figure 6.9: Muon scaled momentum resolution shape function.

In this case, we find the shape function to be the sum of three Gaussians. We define the shape function as before, parameterized by the normalization, mean and width of the nar- 
rowest Gaussian. As before, we fit the $\delta \mathrm{p} / \mathrm{p}$ distribution in bins of both momentum and angle to the shape function, and use the fit results to determine the angular and momentum dependencies of the fit function.
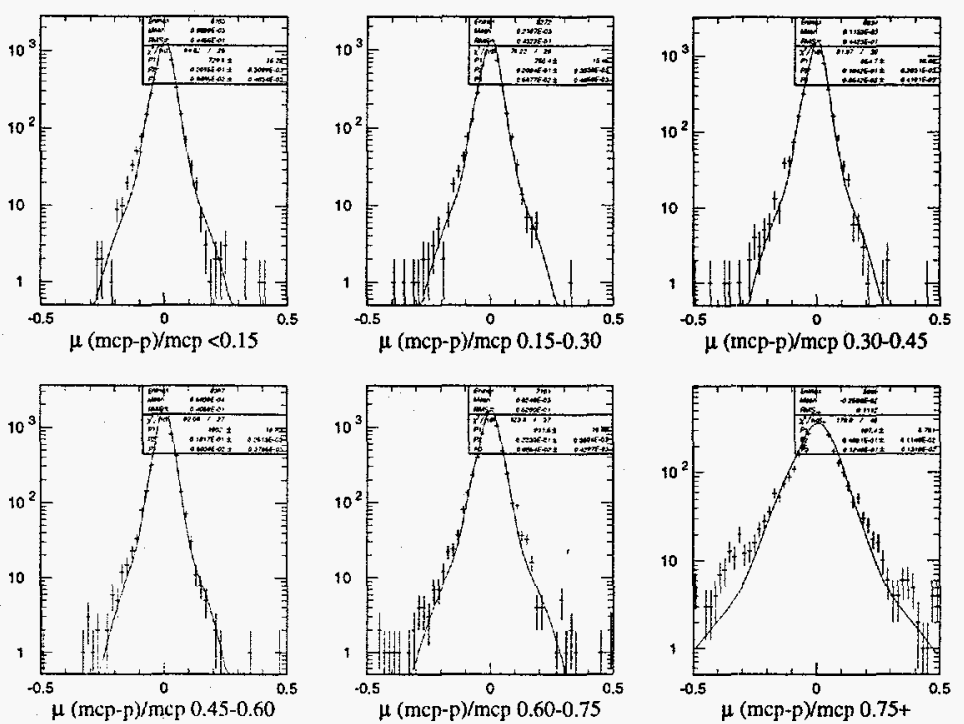

Figure 6.10: Muon momentum resolution fits in bins of $|\cos \theta|$.
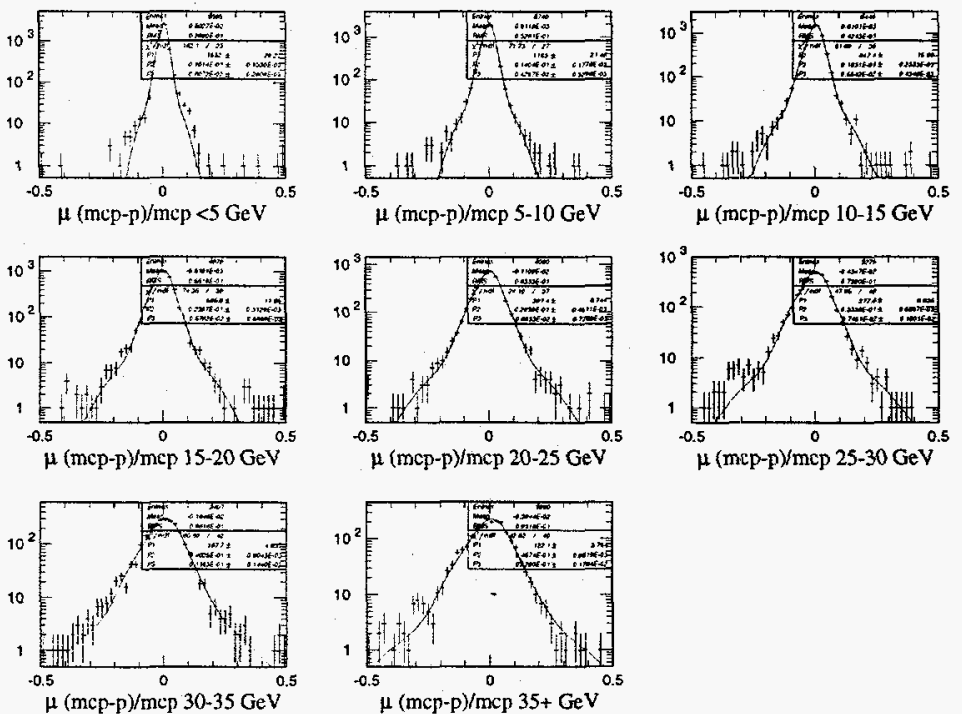

Figure 6.11: Muon momentum resolution fits in momentum bins. 

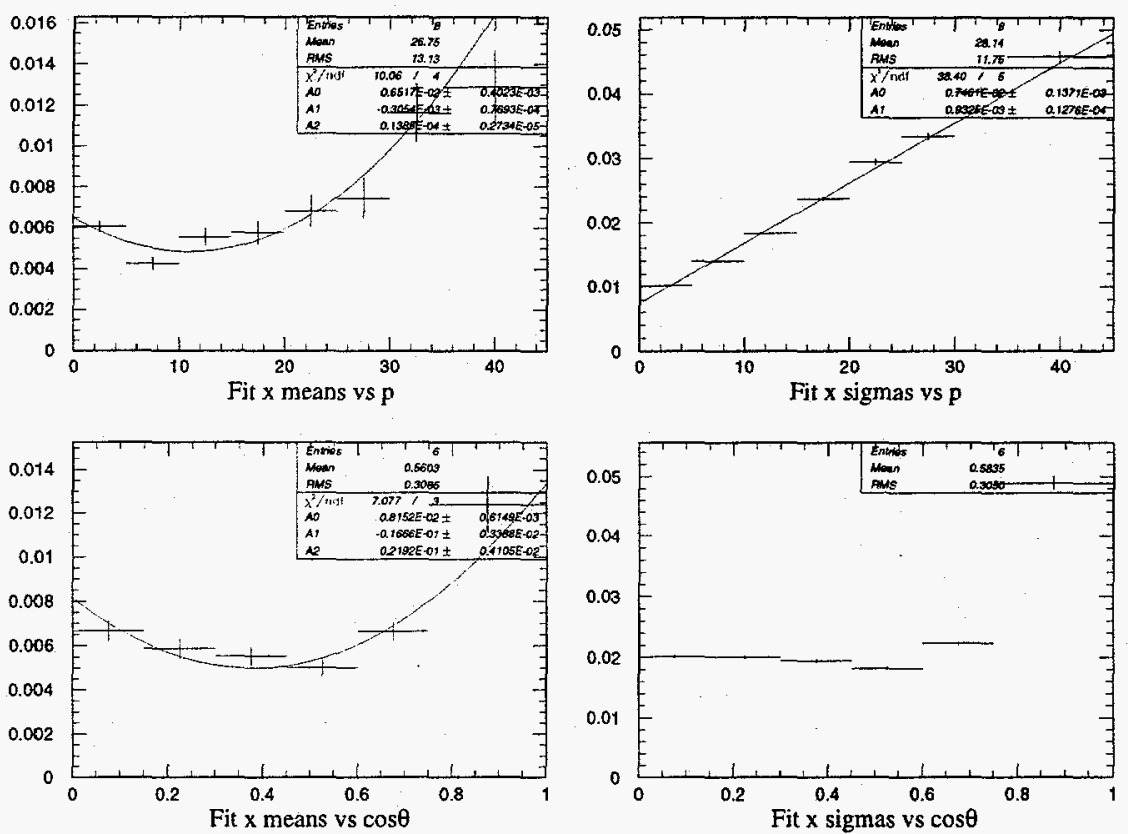

Figure 6.12: Muon momentum resolution fit parameters.

In this case, we find a linear dependence for the width on the momentum, and a weak dependence of the width on angle, which we neglect. We find that the means are quadratically dependent on both angle and momentum, but for the region $|\cos \theta|<0.75$, we find that the angular dependence is also consistent with being flat, so we neglect the angular dependence. This allows us to construct the resolution function as we did for the angle. One important difference is that the shape function is now a function of $\delta p / p$. We must therefore, make the correct change of variables to get the distribution in true momentum.

$$
\mathrm{f}\left(\frac{\delta \mathrm{p}}{\mathrm{p}}\right) \rightarrow \mathrm{f}\left(\frac{\delta \mathrm{p}}{\mathrm{p}}\right)\left|\frac{\mathrm{d} \frac{\delta \mathrm{p}}{\mathrm{p}}}{\mathrm{dp}_{\text {meas }}}\right|=\frac{1}{\mathrm{p}_{\text {true }}} \cdot \mathrm{f}\left(\frac{\mathrm{p}_{\text {true }}-\mathrm{p}_{\text {meas }}}{\mathrm{p}_{\text {true }}}\right)
$$




\subsubsection{Electron Momentum Resolution}

Unfortunately, we cannot determine an electron momentum resolution function using a procedure analogous to the above because the assumption that there is an overall shape function that only varies in width and mean does not hold for the electrons. Due to bremsstrahlung in various materials in the detector, there is a long radiative tail in the electron $\delta \mathrm{p} / \mathrm{p}$ distribution that changes shape for different ellectron momentum ranges. As a result, we must construct a shape function that varies in shape as a function of the electron momentum. We start this procedure by fitting the sum of three independent Gaussians to the electron $\delta p / p$ distribution for different electron energy ranges.
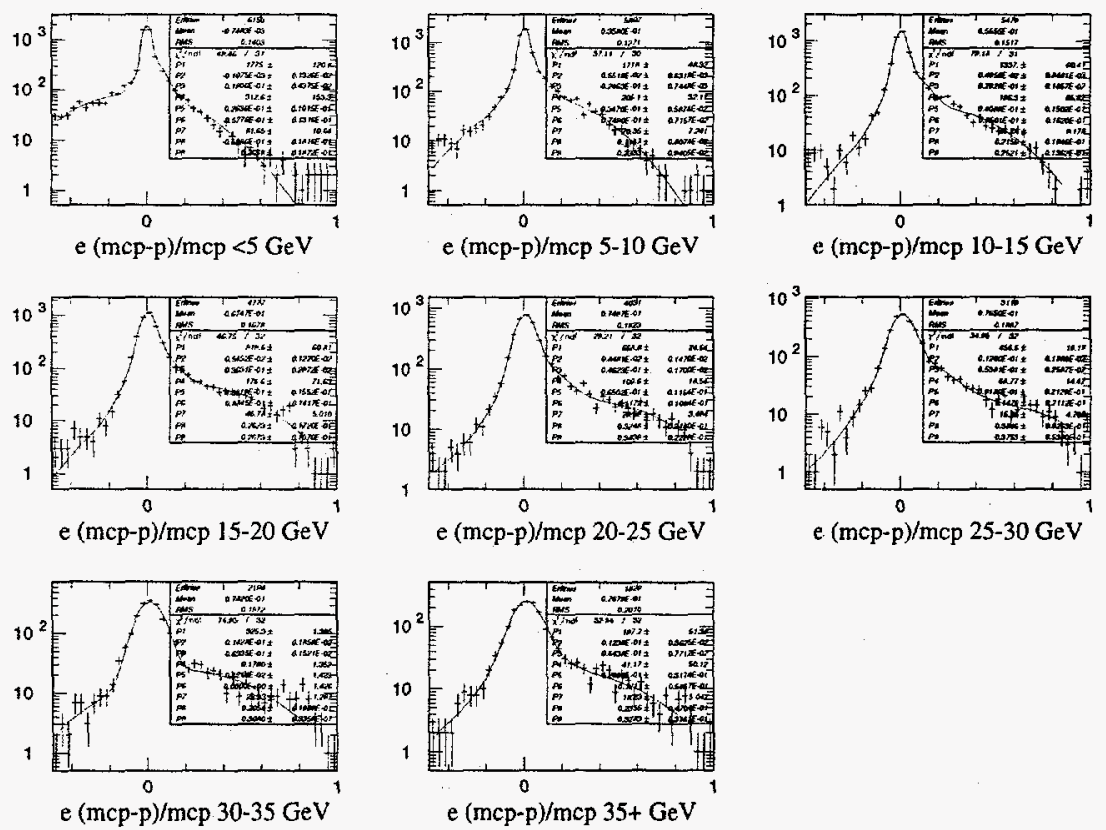

Figure 6.13: Electron momentum resolution shape fits in momentum bins.

We can then plot the resulting fit parameters versus momentum and determine their momentum dependence. 

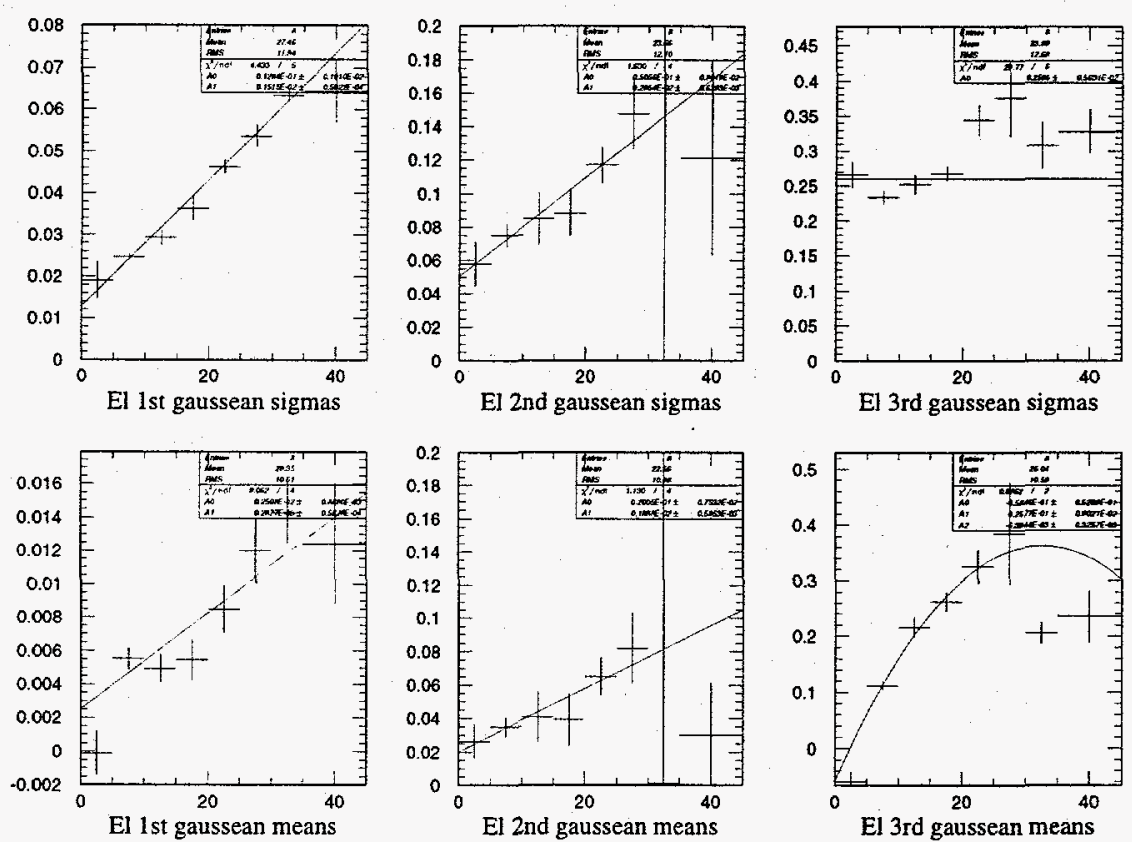

Figure 6.14: Electron resolution shape parameters.

We can then use the parameterizations of the means and sigmas of the three Gaussians to construct a momentum dependent shape function. We have been somewhat arbitrary excluding some of the fit results from the parameterizations, but the final aim is to have a relatively simple parameterization of the resolution function that is more accurate than merely using a delta function or fixed Gaussian. With three independent Gaussians, the relative fractions in the three curves are extremely variable, so in order to determine their variation with momentum, we fix the means and widths of the three Gaussians to the above parameterization and refit the various $\delta \mathrm{p} / \mathrm{p}$ distributions, allowing only the relative normalizations of the three Gaussians to float (Fig. 6.15). 

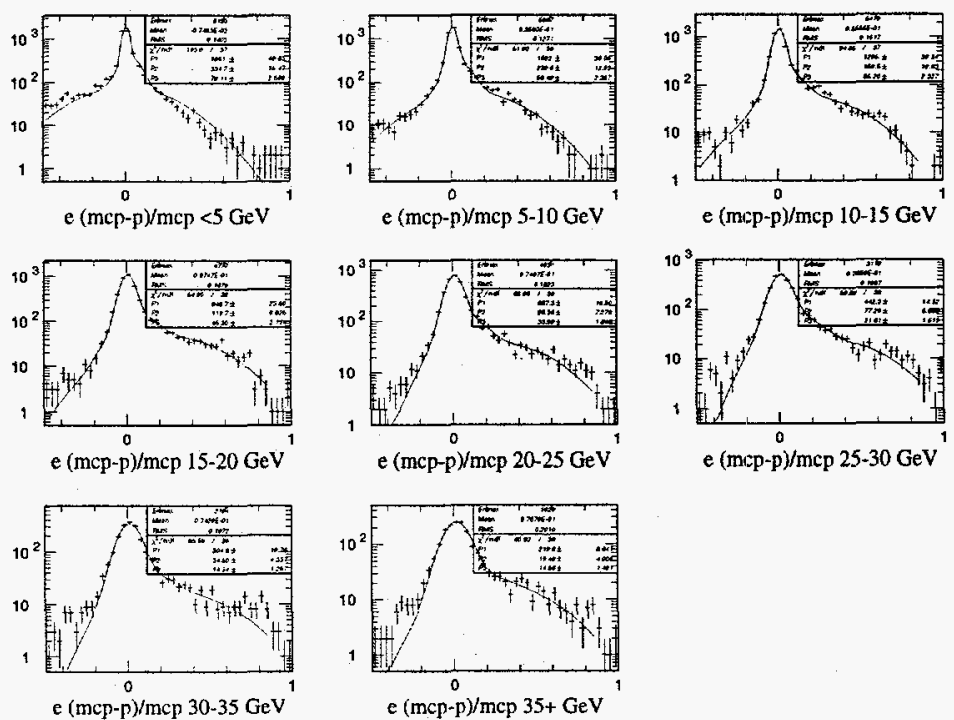

Figure 6.15: Electron momentum resolution fits in momentum bins.
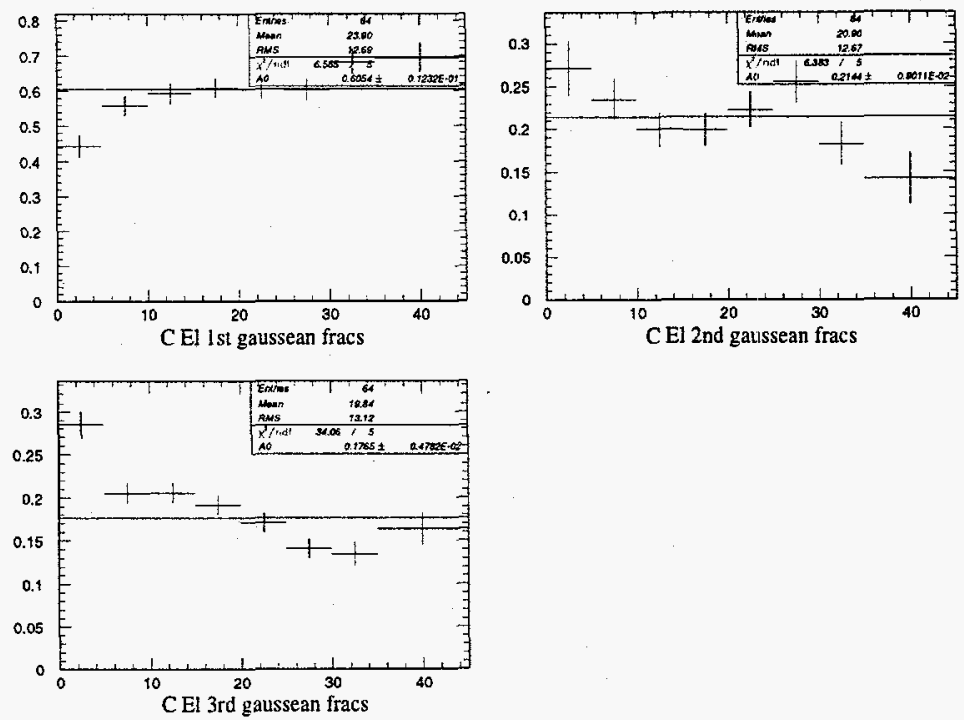

Figure 6.16: Electron momentum resolution fractions.

From our trial Michel parameter fits with various electron momentum resolution functions, we find that it is most important to model the electron resolution at low momentum 
well. As a result, we make a special case for the low momentum bin and use a separate set of Gaussian fractions for that region, and use the overall average for the higher momentum events. Due to the complexity of this procedure and the resulting shape function, it is difficult to incorporate angular effects into the resolution function. As a cross check, we fit the several angular bins with the momentum dependent shape function, using an average momentum of $18 \mathrm{GeV}$. This gives us
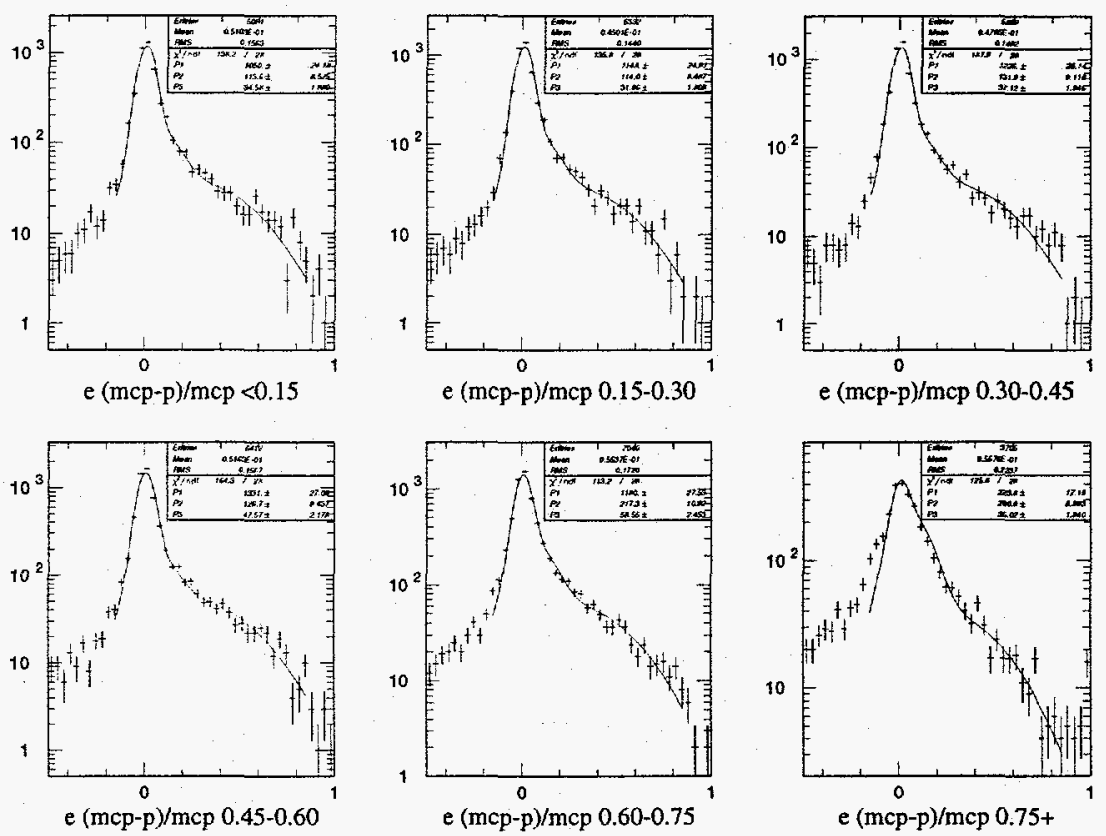

Figure 6.17: Electron momentum resolution in $|\cos \theta|$ bins.

These results appear to be reasonably consistent. The low tail in this distribution is not modeled well by using the shape for the average momentum because the low momentum events contribute a greater tail than the average would predict, so we have cut off that part of the fit. It appears that there is no strong angular dependence of the shape function in this 
case. We construct the resolution function as we did for the muon momentum case, making a change of variables to get a distribution in true momentum.

\subsubsection{Selection Efficiency}

The selection efficiency is the fraction of tracked events that pass the tau selection and decay identification criteria. Because all of the events are tracked, we can use the measured momentum and angle to parameterize these functions and all of the following correction functions. Because the tau selection uses some energy dependent cuts, there are significant differences in the selection efficiency between left-handed and right-handed taus. As a result we parameterize these corrections separately for the two tau helicity states.
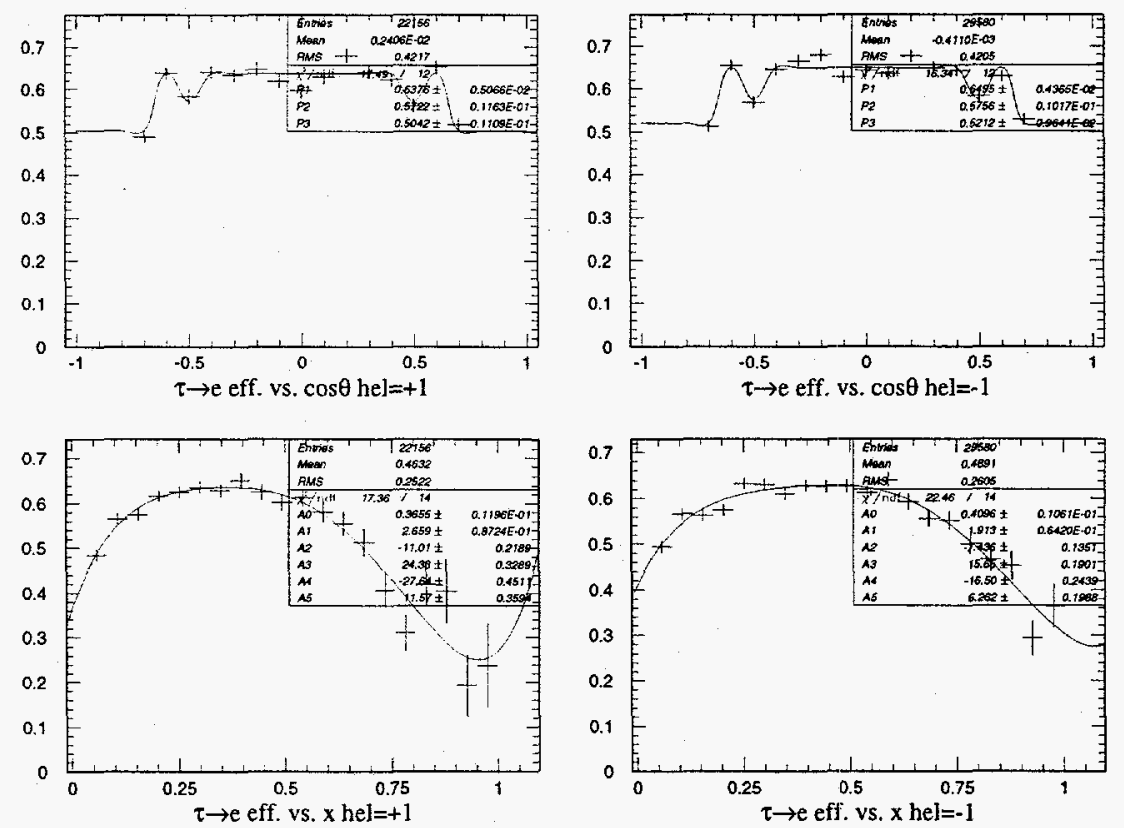

Figure 6.18: Electron selection efficiency. 

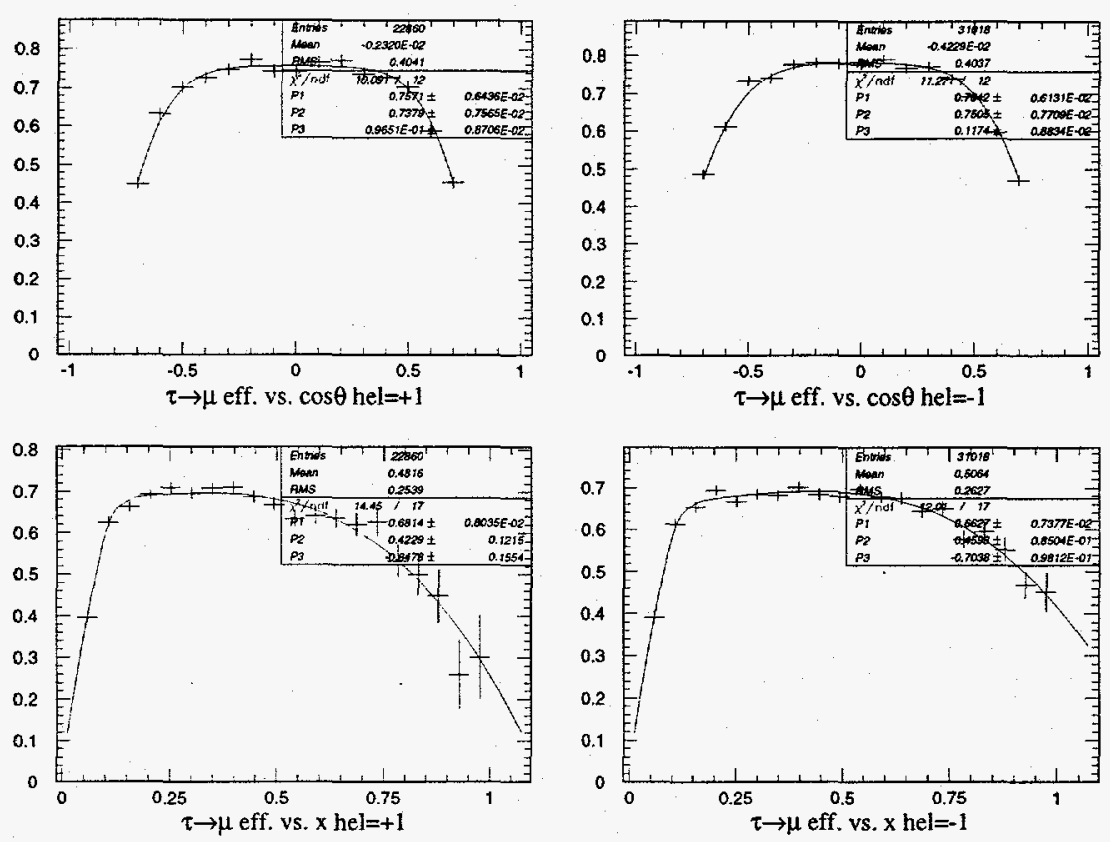

Figure 6.19: Muon selection efficiency.

For the electrons (Fig. 6.18) we parameterize the momentum dependence of the selection efficiency as a fifth order polynomial. We parameterize the angular dependence in three bins $0.75>|\cos \theta|>0.65625,0.5625>|\cos \theta|>0.46875$ and the remaining regions. The plotted fits in the efficiency plots don't do this justice as PAW does a cubic spline interpolation of all functions that it plots. The edges of these bins are chosen to speed up the computation of the numerical integration. The dip is due to the LAC support washer. There is clearly some further structure, but since this is a function of angle, it has negligible effect on the results. We calculate the overall efficiency by taking the product of the two efficiencies and dividing by the overall efficiency, which we find to be 0.542 for the right-handed taus and 0.551 for the left-handed taus. 
For the muons (Fig. 6.19) we parameterize the angular dependence with a function of the form of equation 6.12 . We parameterize the momentum dependence with the form

$$
\varepsilon_{\mathrm{sel}}(\mathrm{x})=\frac{1}{1+\mathrm{e}^{\left(\frac{0.05-\mathrm{x}}{0.025}\right)}}\left(\mathrm{p} 1+\mathrm{p} 2 \cdot \mathrm{x}+\mathrm{p} 3 \cdot \mathrm{x}^{2}\right)
$$

We calculate the overall efficiency by taking the product of the two efficiencies and dividing by the overall efficiency, which we find to be 0.601 for the right-handed taus and 0.614 for the left-handed taus.

\subsubsection{Tau Related Backgrounds}

Mis-identified taus are an important correction in spite of their small magnitude in the event sample. All tau decays are spin dependent so that effects similar to those that we are trying to measure also affect the shapes of the background spectra therefore, we must parameterize these corrections separately for left and right-handed taus.
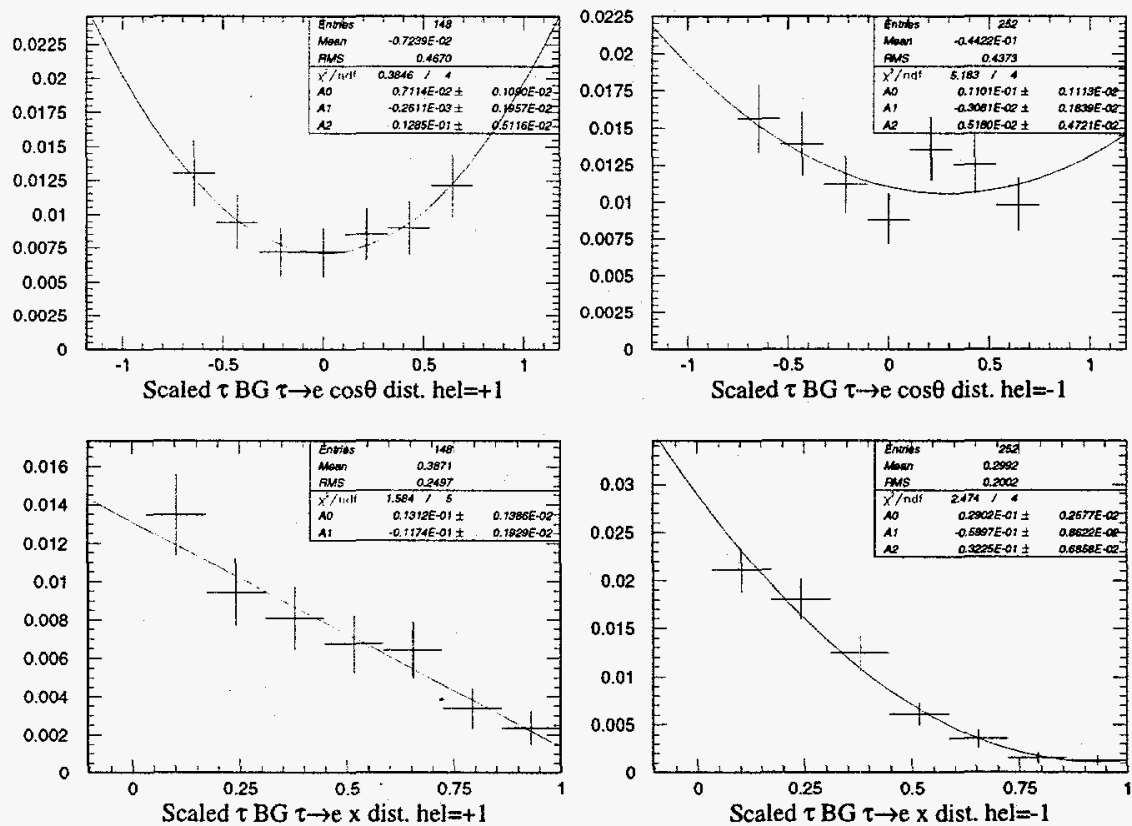

Figure 6.20: Electron tau background. 

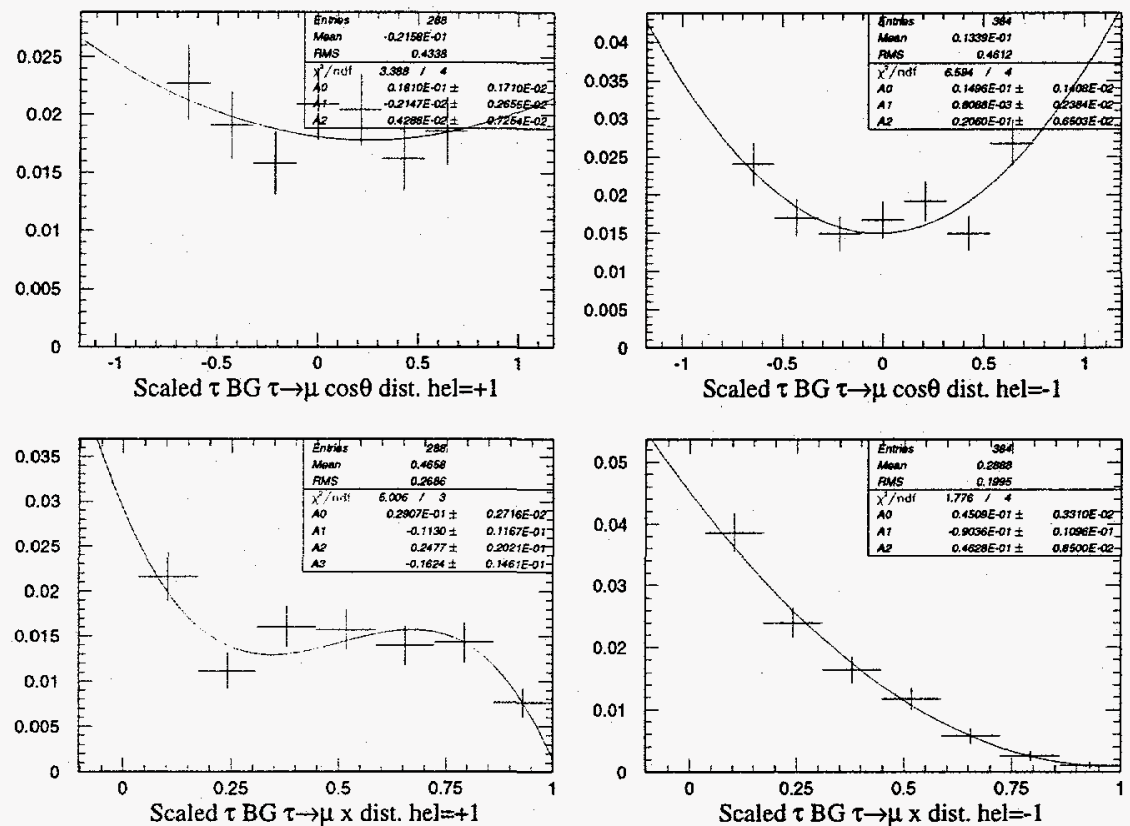

Figure 6.21: Muon tau background.

In all cases, we have parameterized the backgrounds with polynomials. For the angular dependences, we use the shape of the tau pair production cross section rather than the fits, as these fits are consistent with that shape. Care must be taken to make sure that these background functions are normalized properly. We have normalized the theoretical distribution so that when it is integrated over angle and scaled momentum and summed over the two beam polarizations, it comes out to one. The resolution functions are distributions, so the corrected theoretical distribution without background should integrate over angle and momentum and sum over beam polarization to the overall efficiency for correctly selecting and identifying the appropriate decay. Thus, the background functions should integrate and sum to the number of background events we expect to get per tau produced with the appropriate decay. This is accomplished in this case by using the actual generated number 
of decays in the Monte Carlo sample and in the non-tau case by careful application of the cross sections for the various processes.

\subsubsection{Non-Tau Background}

The non-tau background correction is determined using the various Monte Carlo samples for background processes. We must be careful to correctly combine the various sources of background correctly using the calculated cross-sections for the individual processes.
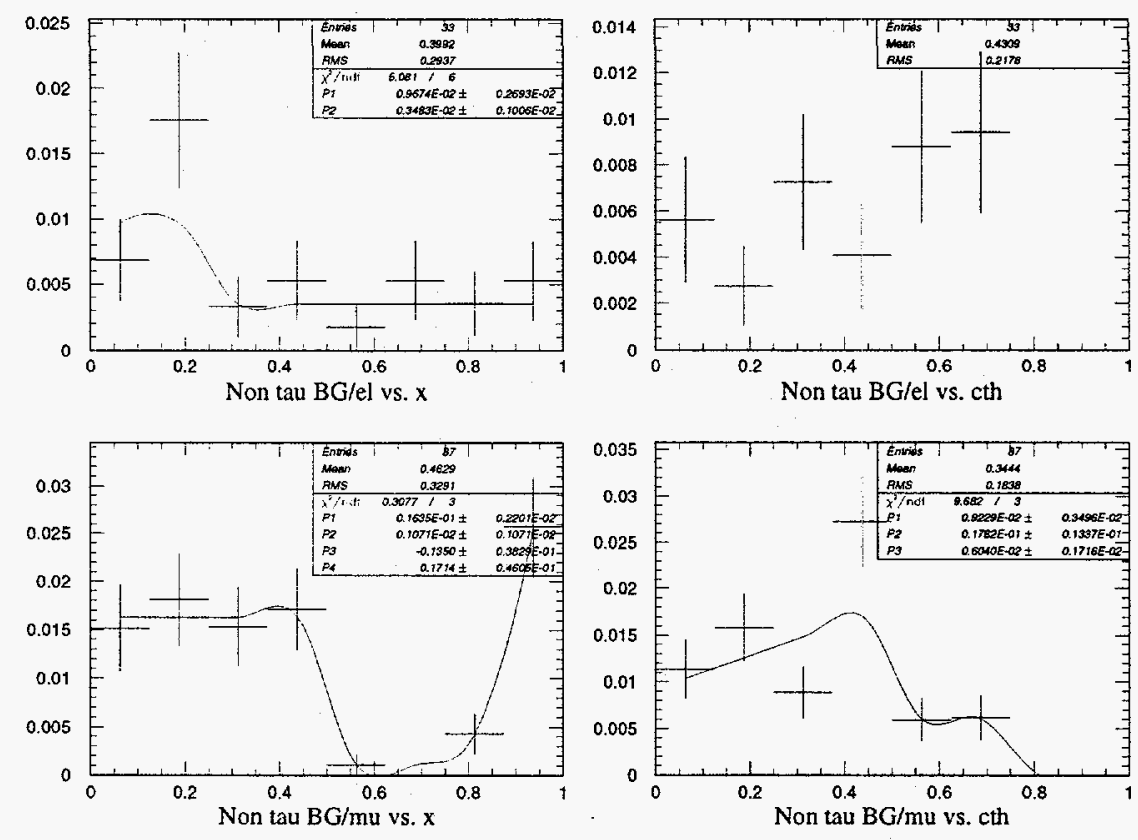

Figure 6.22: Non tau backgrounds vs. $x$ and $\cos \theta$.

In both cases, we use an isotropic distribution in angle as there are no clear angular dependencies in the Monte Carlo background sample. For the electrons, we parameterize the momentum dependence with two constants, one for $\mathrm{x}<0.25$, and other for larger $\mathrm{x}$. For the muons, we parameterize the scaled momentum dependence in three regions, $x<0.5$, $0.5<x<0.75$, and $x>0.75$. The lower two regions have constant levels of background, and 
the upper region has a linearly increasing level of background. The precise boundaries for the $\mathrm{x}$ regions are moved slightly from the above values to speed the evaluation of the numerical integration. 


\section{Chapter 7}

\section{Fit Results and Systematic Errors}

We can now fit the selected data to the corrected theoretical spectrum and extract values for the tau Michel Parameters. In addition, we can study various systematic effects on the fit results and assign uncertainties in the results due to these. Also, we can do a few cross checks to verify that the fit is working as we expect.

\subsection{Another Look at the Data}

To make sure that we have something to fit, we can look at the data distributions and see if the measured energy spectra of leptons from tau decay are affected by tau polarization. Looking at Fig. 1.4, we can see that if we look in the forward region of the detector for left-handed electrons or in the backward region of the detector for right-handed electrons, we get a sample of taus that is mostly left-handed (left-handed enhanced), and looking at the opposite regions, we get a right-handed enhanced sample. We can plot the measured spectra for these two regions (Fig. 7.1) overplotted with a scaled Monte Carlo spectrum. The points are the 1993-95 data, and the histograms are the scaled Monte Carlo sample. The solid points and histogram are the right-handed enhanced taus and the hollow points and dashed histogram are the left-handed enhanced taus. The two spectra are clearly different. The left-handed taus give a significantly harder momentum spectrum than the right-handed ones. We also see that there a numerically more left-handed taus than righthanded taus which is due to the inherent parity violation of the $\mathrm{Z}^{0}$. 


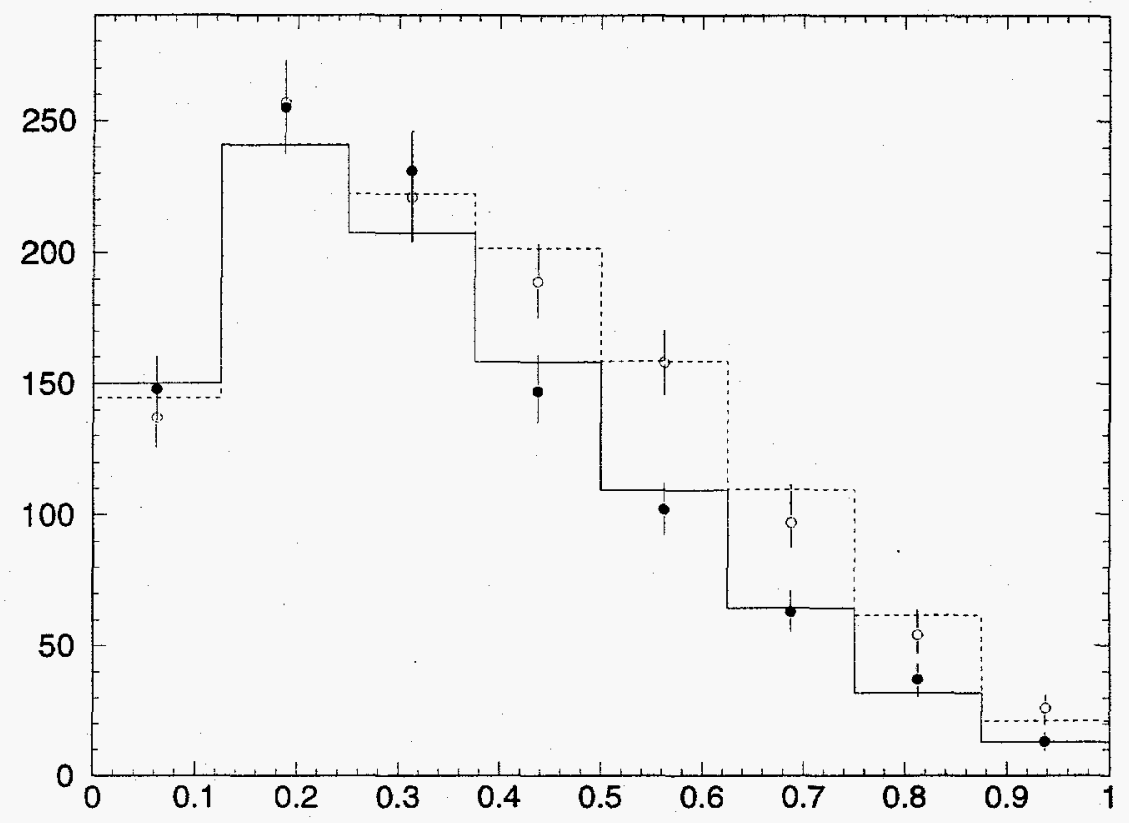

Figure 7.1: Data and Monte Carlo x spectra for left and right handed taus.

\subsection{Fit Results}

We can now confidently apply the fit machinery to the data. We can fit the electrons and muons separately, and we can combine the fits in several different ways.

\subsubsection{Electron Fit}

For the electrons, we do not fit for the $\eta$ parameter, as it enters into the spectrum with a coefficient of $\frac{m_{\ell}}{m_{\tau}}$. The electron mass is about $\frac{1}{3500}$ of the tau mass, so the $\eta$ term in the spectrum will be greatly suppressed. As a result we perform the fit with $\eta$ fixed to 0 , which is the Standard Model expectation for $\eta$. This gives us fit results as follows: 
Table 7.1: Electron Fit Results.

\begin{tabular}{l|r|r}
\hline Parameter & Result & \multicolumn{1}{l|}{ Error } \\
\hline$\rho$ & 0.71 & 0.14 \\
\hline$\xi$ & 1.16 & 0.52 \\
\hline$\xi \delta$ & 0.85 & 0.43 \\
\hline
\end{tabular}

The Errors are statistical only and are determined using the MINOS procedure of the CERN MINUIT package. The resulting parameter correlation matrix is

Table 7.2: Electron Fit Correlation Coefficients.

\begin{tabular}{l|r|r|r}
\hline & \multicolumn{1}{|c|}{$\rho$} & \multicolumn{1}{c|}{$\xi$} & \multicolumn{1}{c}{$\xi \delta$} \\
\hline$\rho$ & 1 & -0.177 & -0.107 \\
\hline$\xi$ & -0.177 & 1 & 0.061 \\
\hline$\xi \delta$ & -0.107 & 0.061 & 1 \\
\hline
\end{tabular}

\subsubsection{Muon Fit}

For the muons, we do a four parameter fit including $\eta$. This gives us fit results as follows:

Table 7.3: Muon Fit Results.

\begin{tabular}{l|r|r}
\hline Parameter & Result & \multicolumn{1}{c|}{ Error } \\
\hline$\rho$ & 0.54 & 0.28 \\
\hline$\xi$ & 0.75 & 0.50 \\
\hline$\xi \delta$ & 0.82 & 0.32 \\
\hline$\eta$ & -0.59 & 0.82 \\
\hline
\end{tabular}

The Errors are statistical only and are determined using the MINOS procedure of the CERN MINUIT package. The resulting parameter correlation matrix is 
Table 7.4: Muon Fit Correlation Coefficients.

\begin{tabular}{l|r|r|r|r}
\hline & \multicolumn{1}{|c|}{$\rho$} & \multicolumn{1}{c|}{$\xi$} & $\xi \delta$ & $\eta$ \\
\hline$\rho$ & 1 & 0.421 & 0.291 & 0.923 \\
\hline$\xi$ & 0.421 & 1 & 0.198 & 0.541 \\
\hline$\xi \delta$ & 0.291 & 0.198 & 1 & 0.373 \\
\hline$\eta$ & 0.923 & 0.541 & 0.373 & 1 \\
\hline
\end{tabular}

$\eta$ and $\rho$ are strongly correlated, which degrades the fit results for both, resulting in much larger errors on the $p$ parameter than we get from the electron fit in spite of the fact that the muon sample is larger than the electron sample and is less diluted by resolution effects.

\subsubsection{Combined Fit Results}

There are several ways to combine the two fits. Previous measurements have assumed lepton universality and fit both spectra for all four parameters. This results in improvements for all four parameters, including $\eta$, which benefits from the increase in precision of the $\rho$ measurement. In effect, the electrons are used to measure $\rho$, and the muons are used to measure $\eta$. In turns out that there are very few theoretical models that allow a both nonzero $\eta$ and lepton universality.[35] Thus it may actually be more correct to do a three parameter combined fit, fixing $\eta$ at zero. We will do both

Table 7.5: Combined Fit with Variable Eta.

\begin{tabular}{l|r|r}
\hline Parameter & Result & \multicolumn{1}{l|}{ Error } \\
\hline$\rho$ & 0.69 & 0.13 \\
\hline$\xi$ & 1.02 & 0.36 \\
\hline$\xi \delta$ & 0.87 & 0.27 \\
\hline$\eta$ & -0.13 & 0.47 \\
\hline
\end{tabular}


Table 7.6: Combined Fit with Fixed Eta.

\begin{tabular}{l|r|r}
\hline Parameter & Result & \multicolumn{1}{c}{ Error } \\
\hline$\rho$ & 0.72 & 0.09 \\
\hline$\xi$ & 1.05 & 0.35 \\
\hline$\xi \delta$ & 0.88 & 0.27 \\
\hline
\end{tabular}

Fixing $\eta$ improves the errors on the other measurements by reducing the correlations within the fit. Fortunately, fixing $\eta$ does not significantly affect the other results. The correlation matrices are

Table 7.7: Combined Fit with Variable Eta Correlation Coefficients.

\begin{tabular}{l|r|r|r|r}
\hline & \multicolumn{1}{|c|}{$\rho$} & \multicolumn{1}{|c|}{$\xi$} & $\xi \delta$ & $\eta$ \\
\hline$\rho$ & 1 & 0.057 & 0.026 & 0.689 \\
\hline$\xi$ & 0.057 & 1 & 0.080 & 0.299 \\
\hline$\xi \delta$ & 0.026 & 0.080 & 1 & 0.182 \\
\hline$\eta$ & 0.689 & 0.299 & 0.182 & 1 \\
\hline
\end{tabular}

Table 7.8: Combined Fit with Fixed Eta Correlation Coefficients.

\begin{tabular}{l|r|r|r}
\hline & \multicolumn{1}{|c|}{$\rho$} & \multicolumn{1}{c|}{$\xi$} & $\xi \delta$ \\
\hline$\rho$ & 1 & -0.215 & -0.139 \\
\hline$\xi$ & -0.215 & 1 & 0.030 \\
\hline$\xi \delta$ & -0.139 & 0.030 & 1 \\
\hline
\end{tabular}

\subsection{Systematic Errors}

In addition to the statistical errors coming from the fits, there are many possible sources of uncertainty in the measurement. Foremost among these are uncertainties in the parameterizations of the correction functions, which also reflect uncertainties in the calibration of 
the detector. Also, the fit procedure itself may be biased or incorrect. Several studies have been performed to evaluate the systematic uncertainties of the measurements.

\subsubsection{Errors due to Correction Functions and Input Parameters}

Due to limited Monte Carlo statistics and the imperfection of the Monte Carlo, the parameterizations of the correction functions have uncertainties. Also, several of the input parameters to the fit have uncertainties due to measurement errors. $A_{e}$ and $A_{\tau}$ are imperfectly measured physical quantities that the fit requires. To take into account uncertainties in the correction functions, we redo the fits varying each individual parameterization within its statistical errors. Due to large correlations between some parameters of the correction functions, some care must be taken to ensure that we are not drastically overestimating these systematic uncertainties, particularly in cases where high order polynomials have been used for the parameterization. The resulting variations in the fit results are assigned as the systematic error due to that parameterization. We follow a similar proce-

dure for $A_{e}$ and $A_{\tau}$, where we assume lepton universality at the $Z^{0}$ and use the SLD $A_{\ell}$ value of $0.1542 \pm 0.0037 .[36]$

The beam polarization and beam energy are also measured with some uncertainty on an event by event basis. We do not take these into account in the resolution functions. To account for the beam polarization error, we redo the fits, shifting the magnitudes of all of the measured polarizations upward and downward by the stated error on the polarization, assigning the resulting variation in the fit results as a systematic error. For the beam energy, we follow the same procedure, refitting with the energies shifted upward and downward by their stated uncertainties.

In the case of the radiative corrections, the uncertainties in the parameterization are certainly outweighed by the actual theoretical uncertainty of the KORALZ Monte Carlo due to the exceedingly high statistics of the generator level Monte Carlo sample used to 
determine the correction. We take the systematic error due to the radiative corrections to be $10 \%$ of the difference between the fit values with and without the radiative corrections. This variation is greatly in excess of any variation we expect from the inaccuracy of the KORALZ generator,[37] but provides a good conservative systematic error.

For each result, we assume that the individual systematics are independent and combine the various systematics by adding them in quadrature. For the combined fits, we vary each parameter separately for the two decays and assume that the parameterizations for the two decays are independent of one another. As a result, the systematics for the combined fit are often better than one would expect from combining the two results. The systematics for the combined fit with $\eta$ are substantially higher than those without it due to the large correlations that $\eta$ introduces into the fit. Some of the effects in the following tables actually represent several independent effects. In particular, the systematic effects for the combined fits represent a combination of both the muon and electron systematic errors.

Table 7.9: Combined Fit with Variable Eta Systematics.

\begin{tabular}{l|l|r|r|r}
\hline \multicolumn{1}{c|}{ Effect } & $\rho$ & $\xi$ & $\xi \delta$ & \multicolumn{1}{c}{$\eta$} \\
\hline $\mathrm{A}_{\ell}$ & 0.003 & 0.021 & 0.012 & 0.003 \\
\hline Radiation & 0.004 & 0.002 & 0.002 & 0.009 \\
\hline Beam Polarization & 0.001 & 0.009 & 0.005 & 0.002 \\
\hline Beam Energy & 0.013 & 0.013 & 0.003 & 0.003 \\
\hline Non- $\tau$ Background & 0.021 & 0.022 & 0.011 & 0.099 \\
\hline$\tau$ Background & 0.009 & 0.007 & 0.006 & 0.036 \\
\hline Event Selection & 0.023 & 0.011 & 0.018 & 0.066 \\
\hline Momentum Resolution & 0.033 & 0.027 & 0.030 & 0.092 \\
\hline Angular Resolution & 0.000 & 0.001 & 0.000 & 0.002 \\
\hline Total & 0.048 & 0.046 & 0.040 & 0.155 \\
\hline
\end{tabular}


Table 7.10: Combined Fit with Fixed Eta Systematics.

\begin{tabular}{l|l|l|l}
\hline \multicolumn{1}{c|}{ Effect } & \multicolumn{1}{c|}{$\rho$} & $\xi$ & $\xi \delta$ \\
\hline $\mathrm{A}_{\ell}$ & 0.003 & 0.020 & 0.014 \\
\hline Radiation & 0.003 & 0.002 & 0.001 \\
\hline Beam Polarization & 0.000 & 0.008 & 0.005 \\
\hline Beam Energy & 0.014 & 0.006 & 0.006 \\
\hline Non- $\tau$ Background & 0.007 & 0.004 & 0.004 \\
\hline$\tau$ Background & 0.005 & 0.007 & 0.005 \\
\hline Event Selection & 0.011 & 0.013 & 0.017 \\
\hline Momentum Resolution & 0.019 & 0.025 & 0.027 \\
\hline Angular Resolution & 0.000 & 0.001 & 0.000 \\
\hline Total & 0.028 & 0.037 & 0.036 \\
\hline
\end{tabular}

Table 7.11: Electron Fit Systematics.

\begin{tabular}{l|c|c|c}
\hline \multicolumn{1}{c|}{ Effect } & $\rho$ & $\xi$ & $\xi \delta$ \\
\hline $\mathrm{A}_{\ell}$ & 0.003 & 0.016 & 0.016 \\
\hline Radiation & 0.004 & 0.002 & 0.002 \\
\hline Beam Polarization & 0.000 & 0.008 & 0.005 \\
\hline Beam Energy & 0.016 & 0.005 & 0.005 \\
\hline Non- $\tau$ Background & 0.009 & 0.004 & 0.002 \\
\hline$\tau$ Background & 0.008 & 0.010 & 0.007 \\
\hline Event Selection & 0.027 & 0.026 & 0.044 \\
\hline Momentum Resolution & 0.038 & 0.049 & 0.059 \\
\hline Angular Resolution & 0.000 & 0.001 & 0.000 \\
\hline Total & 0.051 & 0.059 & 0.076 \\
\hline
\end{tabular}


Table 7.12: Muon Fit Systematics.

\begin{tabular}{l|r|r|r|r}
\hline \multicolumn{1}{c|}{ Effect } & $\rho$ & $\xi$ & $\xi \delta$ & $\eta$ \\
\hline $\mathrm{A}_{\ell}$ & 0.002 & 0.024 & 0.008 & 0.014 \\
\hline Radiation & 0.004 & 0.001 & 0.001 & 0.008 \\
\hline Beam Polarization & 0.003 & 0.009 & 0.004 & 0.008 \\
\hline Beam Energy & 0.003 & 0.019 & 0.001 & 0.044 \\
\hline Non- $\tau$ Background & 0.127 & 0.126 & 0.060 & 0.397 \\
\hline$\tau$ Background & 0.041 & 0.035 & 0.019 & 0.127 \\
\hline Event Selection & 0.045 & 0.043 & 0.020 & 0.138 \\
\hline Momentum Resolution & 0.026 & 0.022 & 0.029 & 0.051 \\
\hline Angular Resolution & 0.002 & 0.001 & 0.000 & 0.006 \\
\hline Total & 0.143 & 0.144 & 0.072 & 0.445 \\
\hline
\end{tabular}

We see that as we would expect, the momentum resolution is one of the dominant sources of systematic error. The selection efficiency is similarly important due primarily to its momentum dependence. For fits involving $\eta$, we see that background, particularly nontau background is very important. This is primarily due to the fact that the $\eta$ term in the spectrum resembles a background function. We also see that the $\eta$ and $\rho$ parameters are relatively unaffected by the beam polarization and $A_{\ell}$ value. We expect this because these parameters describe the spin independent part of the Michel spectrum.

\subsubsection{Generator Level Fit Check}

In order to check the fit procedure, we fit a sample generator level KORALZ events using the fit function corrected for radiation. The sample consisted of 4000 tau pairs generated with $100 \%$ beam polarization, each with one electronic and one muonic decay. This gives us fit results of 
Table 7.13: Generator Level Fit Results.

\begin{tabular}{l|r|r|l}
\hline Parameter & Combined & \multicolumn{1}{c|}{ Electrons } & \multicolumn{1}{l}{ Muons } \\
\hline$\rho$ & $0.78 \pm 0.05$ & $0.78 \pm 0.05$ & $0.74 \pm 0.13$ \\
\hline$\xi$ & $1.07 \pm 0.11$ & $1.12 \pm 0.15$ & $0.99 \pm 0.20$ \\
\hline$\xi \delta$ & $0.72 \pm 0.07$ & $0.65 \pm 0.10$ & $0.79 \pm 0.13$ \\
\hline$\eta$ & $0.05 \pm 0.20$ & fixed & $-0.05 \pm 0.40$ \\
\hline
\end{tabular}

These results agree quite well with the input standard model values.

\subsubsection{Full Monte Carlo Fit Check}

We have enough generated tau Monte Carlo to make 21 samples of events equivalent to the data sample. We can run fits on these 21 samples to check the validity of the fit process. Note that this study and the following toy Monte Carlo studies were done before we decided to allow $\eta$ to vary in the fit, thus they have been done entirely with $\eta$ fixed at 0 .
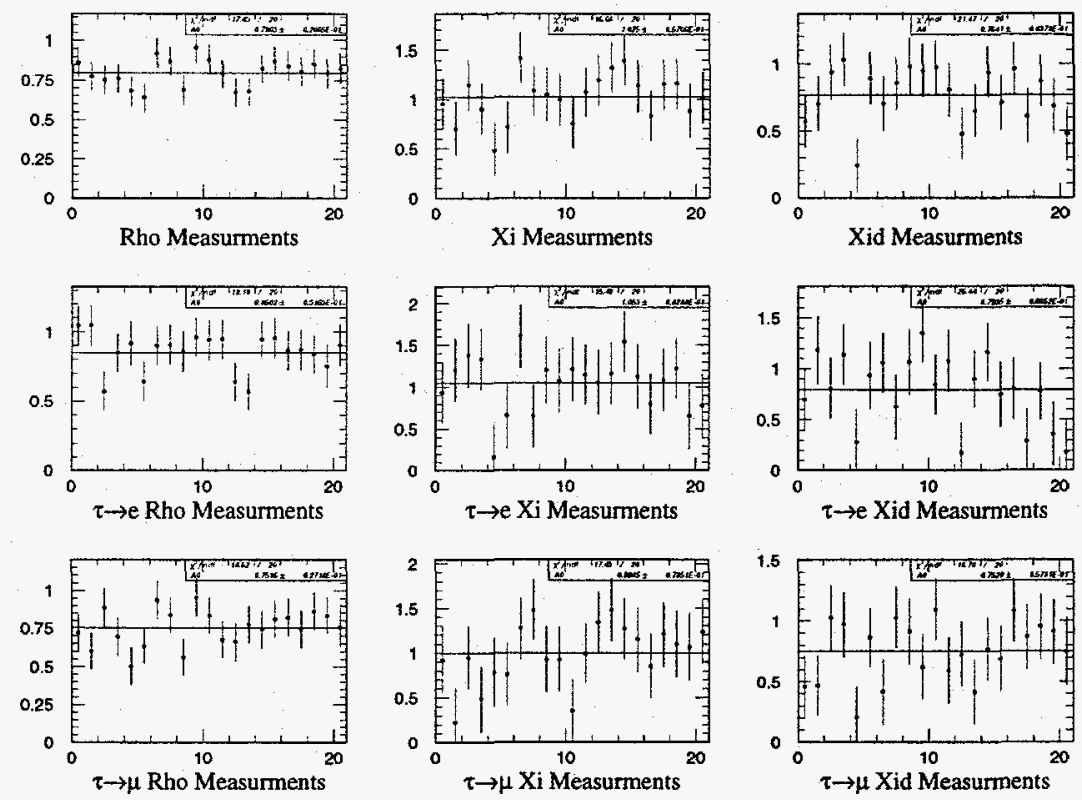

Figure 7.2: Fit results for 21 Monte Carlo samples. 
These fit results agree reasonably well with the input Standard Model values. The electron $\rho$ fit may be returning values a little high, but these are not too inconsistent with the electron $\rho$ systematic error. It looks like the fit procedure is doing a good job of fitting the true Monte Carlo spectra.

\subsubsection{Toy Monte Carlo Studies}

In order to study the effects of nonstandard values of the Michel parameters and to do high statistics studies of the fit method, we have written a toy Monte Carlo program to generate samples of data with distributions as predicted by the corrected theoretical distribution. This program uses the same corrected theoretical spectrum used by the fitter to generate event samples with arbitrary input parameters. We have used this to see how well the fit returns nonstandard values, and to see the accuracy of the statistical errors reported by MINUIT.

We find that the fitter does an excellent job of reproducing nonstandard values for the Michel parameters. This is not too unexpected for this test, as we have effectively stacked the deck by using the same theoretical distribution to produce and fit the data, but it does indicate that there are no extreme biases in the fit procedure.

We generated 1000 samples of toy Monte Carlo data equivalent to the data sample and ran the fits on them. We then compared the actual scatter in the fit results with the errors reported by MINUIT for the fit.

Table 7.14: Toy MC Fit Results.

\begin{tabular}{l|r|r|r}
\hline Parameter & Mean result & Mean Error & True spread \\
\hline$\rho$ & 0.750 & 0.095 & 0.096 \\
\hline$\xi$ & 0.988 & 0.341 & 0.324 \\
\hline$\xi \delta$ & 0.742 & 0.267 & 0.272 \\
\hline
\end{tabular}


It seems that MINUIT's statistical errors are correct, although it might be overestimating the errors on $\xi$. We assign no additional systematic error due to the fit technique.

\subsubsection{Fits on Subsamples}

We can check the consistency of the fit by breaking the data into various subsamples and refitting for each one. We look at the different running periods, as well as the different charged taus. This study was done for a slightly older version of the fitting program and the SLD reconstruction than the one used for the final results reported here. As a result, the results are slightly different, including a fixed $\eta$ for all fits. Very little has changed since then, so we expect that the conclusions regarding the consistency of the fit are sill valid. Looking at the results, they seem nicely consistent.

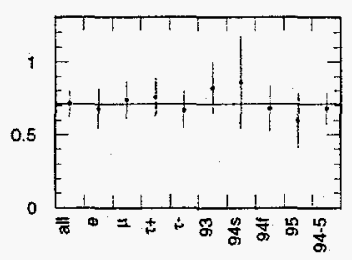

Rho Measurments

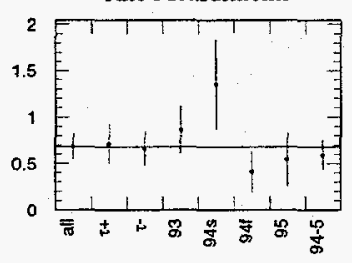

$\tau \rightarrow \mathrm{e}$ Rho Measurments

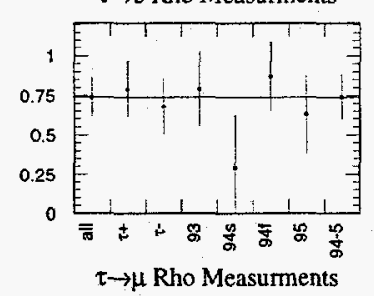

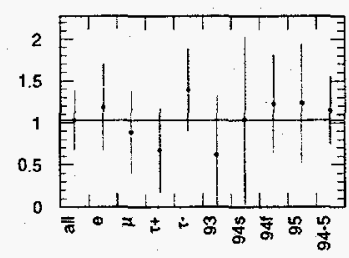

Xi Measurments

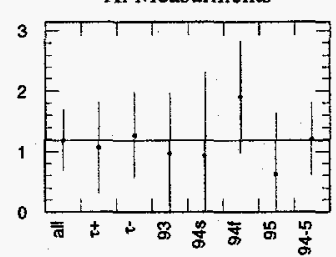

$\tau \rightarrow \mathrm{e}$ Xi Measurments

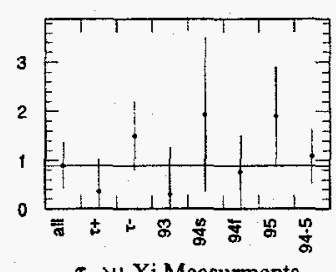

$\tau \rightarrow \mu \mathrm{Xi}$ Measurments

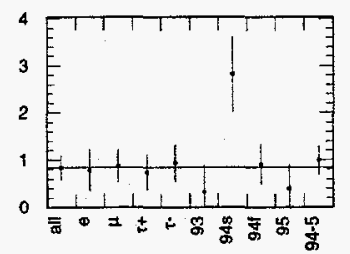

Xid Measurments
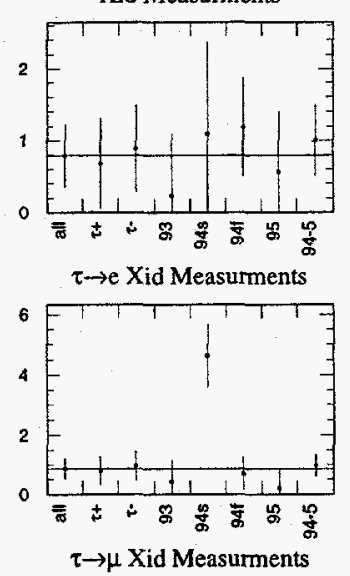

Figure 7.3: Fit results for different subsamples of the data. 


\subsubsection{Resolution Studies using the Data}

We can check the resolution functions by looking at data events with electrons or muons that have known momenta. At the $Z^{0}$ resonance, the only accessible source of these is mupairs for the muons and wide angle Bhabhas for the electrons. This is unfortunate in that these all have momenta equal to the beam energy, so we cannot study the momentum dependence of the resolution functions. Actually, in the case of the WAB events, the tracks can have different energies in radiative events, but measuring this difference is not particularly accurate and the sample is small.

We select a sample of mu-pairs by requiring the events to have two oppositely charged tracks, one in each hemisphere, a total electromagnetic energy less than $30 \mathrm{GeV}$, an event mass calculated from the track momenta greater than $50 \mathrm{GeV}$, and each track identified as a muon by MUSTAT. We can then plot $\frac{E_{\text {beam }}-p}{E_{\text {beam }}}$ for these events.

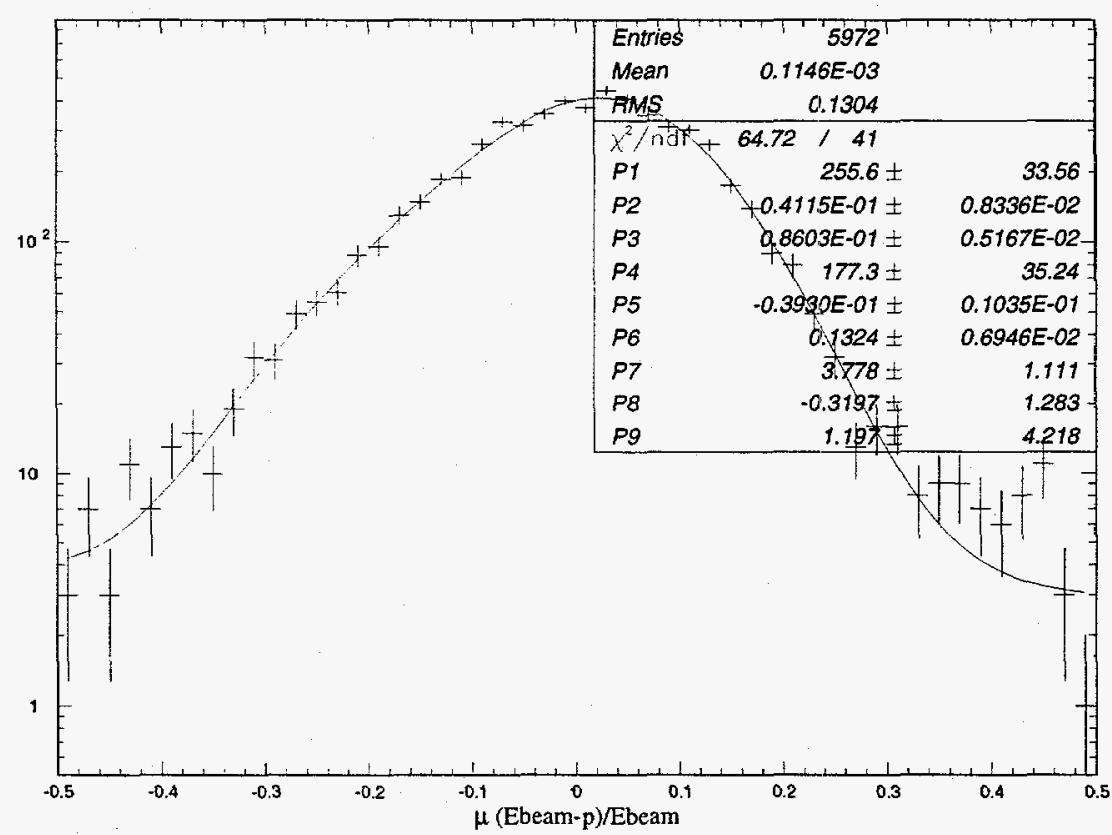

Figure 7.4: Mu pair resolution from data. 
This is significantly different from the Monte Carlo based resolution function. The distribution is significantly wider and offset from zero. Also, the tails of the distribution are significantly larger. The offset is probably due to initial state radiation, which will reduce the interaction center of mass energy from the true center of mass energy. If we use this shape in place of the muon shape function and keep the energy and angular dependence of the width and mean of the shape as determined from the Monte Carlo and redo the fits, we find that the results change within the bounds specified by the resolution systematic errors.

We use the sample of WAB events from the data from Appendix C. We plot $\frac{E_{\text {beam }}-p}{E_{\text {beam }}}$ for these events, where we add in nearby unassociated clusters to the track momentum as we do for the electronic tau decays.

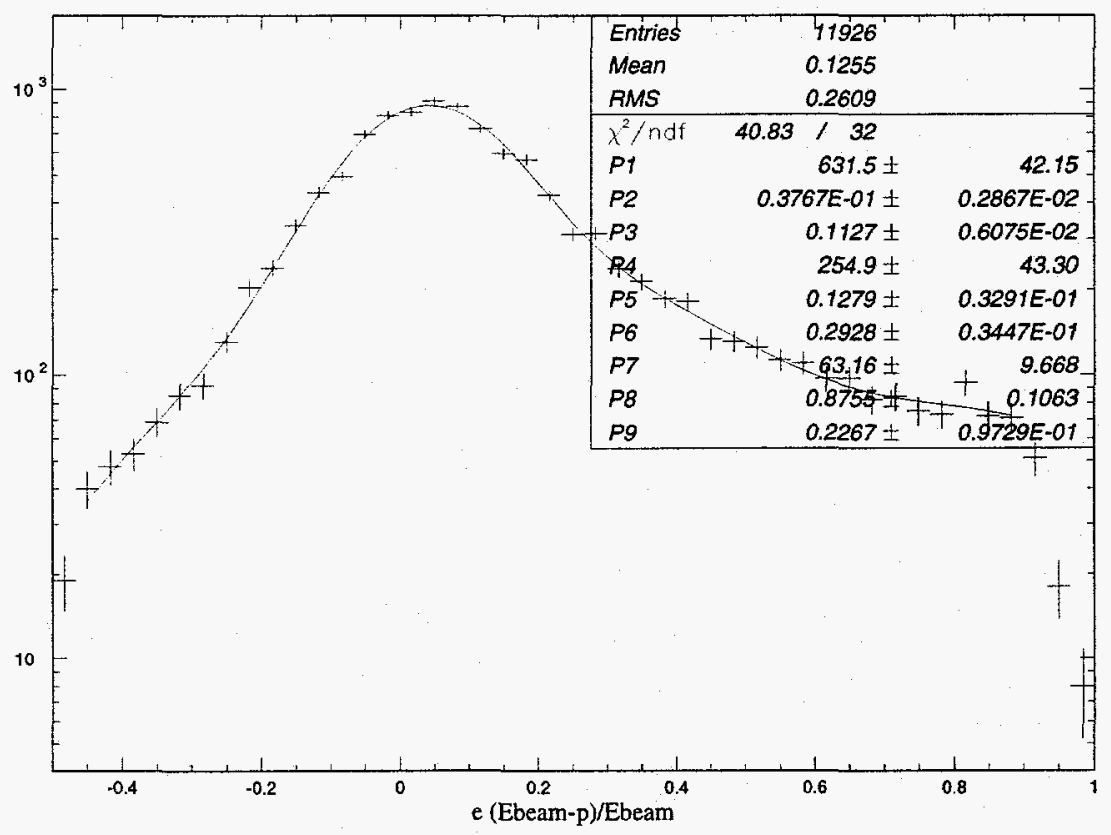

Figure 7.5: WAB resolution from data. 
Again this is significantly different from the Monte Carlo resolution function. We can substitute this shape for the Monte Carlo shape for high momenta, and assume that the widths at lower momenta vary as the Monte Carlo predicts but are rescaled to give this distribution at high momenta and redo the fit. Again the fit results stay within the bounds of the resolution systematic errors.

\subsection{Summery of Results}

Combining the fit results and systematic errors, we get the following results for the tau Michel parameters.

Table 7.15: Summery of Results.

\begin{tabular}{l|c|r|r|c|c}
\hline & Combined w/ $\eta$ & Combined & Electrons & Muons & SM \\
\hline$\rho$ & $0.69 \pm 0.13 \pm 0.05$ & $0.72 \pm 0.09 \pm 0.03$ & $0.71 \pm 0.14 \pm 0.05$ & $0.54 \pm 0.28 \pm 0.14$ & $\frac{3}{4}$ \\
\hline$\xi$ & $1.02 \pm 0.36 \pm 0.05$ & $1.05 \pm 0.35 \pm 0.04$ & $1.16 \pm 0.52 \pm 0.06$ & $0.75 \pm 0.50 \pm 0.14$ & 1 \\
\hline$\xi \delta$ & $0.87 \pm 0.27 \pm 0.04$ & $0.88 \pm 0.27 \pm 0.04$ & $0.85 \pm 0.43 \pm 0.08$ & $0.82 \pm 0.32 \pm 0.07$ & $\frac{3}{4}$ \\
\hline$\eta$ & $-0.13 \pm 0.47 \pm 0.16$ & fixed at 0 & fixed at 0 & $-0.59 \pm 0.82 \pm 0.45$ & 0 \\
\hline
\end{tabular}

In all cases the first error is the statistical and the second the systematic. These results are quite consistent with the standard model and with previous measurements of these quantities.[7][8]-[11] These results are the first results to measure the sign of $\xi$ and $\xi \delta$. These results will be published in [38]. 


\section{Appendix A}

\section{The SLD Collaboration}

K. Abe, ${ }^{(18)}$ K. Abe, ${ }^{(28)}$ I. Abt, ${ }^{(13)}$ T. Akagi, ${ }^{(26)}$ N.J. Allen, ${ }^{(4)}$ W.W. Ash, ${ }^{(26) \dagger}$ D. Aston, ${ }^{(26)}$

K.G. Baird, ${ }^{(23)}$ C. Baltay, ${ }^{(32)}$ H.R. Band, ${ }^{(31)}$ M.B. Barakat, ${ }^{(32)}$ G. Baranko, ${ }^{(9)}$.

O. Bardon, ${ }^{(15)}$ T. Barklow, ${ }^{(26)}$ A.O. Bazarko, ${ }^{(10)}$ R. Ben-David, ${ }^{(32)}$ A.C. Benvenuti, ${ }^{(2)}$

G.M. Bilei, ${ }^{(21)}$ D. Bisello, ${ }^{(20)}$ G. Blaylock, ${ }^{(6)}$ J.R. Bogart, ${ }^{(26)}$ B. Bolen, ${ }^{(17)}$ T. Bolton, ${ }^{(10)}$

G.R. Bower, ${ }^{(26)}$ J.E. Brau, ${ }^{(19)}$ M. Breidenbach, ${ }^{(26)}$ W.M. Bugg, ${ }^{(27)}$ D. Burke, ${ }^{(26)}$

T.H. Burnett, ${ }^{(30)}$ P.N. Burrows, ${ }^{(15)}$ W. Busza, ${ }^{(15)}$ A. Calcaterra, ${ }^{(12)}$ D.O. Caldwell, (5)

D. Calloway, ${ }^{(26)}$ B. Camanzi, ${ }^{(11)}$ M. Carpinelli, ${ }^{(22)}$ R. Cassell, ${ }^{(26)}$ R. Castaldi, ${ }^{(22)(a)}$

A. Castro, ${ }^{(20)}$ M. Cavalli-Sforza, ${ }^{(6)}$ A. Chou, ${ }^{(26)}$ E. Church, ${ }^{(30)}$ H.O. Cohn, ${ }^{(27)}$

J.A. Coller, ${ }^{(3)}$ V. Cook, ${ }^{(30)}$ R. Cotton, ${ }^{(4)}$ R.F. Cowan, ${ }^{(15)}$ D.G. Coyne, ${ }^{(6)}$ G. Crawford, ${ }^{(26)}$

A. D'Oliveira, ${ }^{(7)}$ C.J.S. Damerell, ${ }^{(24)}$ M. Daoudi, ${ }^{(26)}$ R. De Sangro, ${ }^{(12)}$ R. Dell'Orso, ${ }^{(22)}$

P.J. Dervan, ${ }^{(4)}$ M. Dima, ${ }^{(8)}$ D.N. Dong, ${ }^{(15)}$ P.Y.C. Du, ${ }^{(27)}$ R. Dubois, ${ }^{(26)}$

B.I. Eisenstein, ${ }^{(13)}$ R. Elia, ${ }^{(26)}$ E. Etzion, ${ }^{(4)}$ D. Falciai, ${ }^{(21)}$ C. Fan, ${ }^{(9)}$ M.J. Fero, ${ }^{(15)}$

R. Frey, ${ }^{(19)}$ K. Furuno, ${ }^{(19)}$ T. Gillman, ${ }^{(24)}$ G. Gladding, ${ }^{(13)}$ S. Gonzalez, ${ }^{(15)}$

G.D. Hallewell, ${ }^{(26)}$ E.L. Hart, ${ }^{(27)}$ J.L. Harton, ${ }^{(8)}$ A. Hasan, ${ }^{(4)}$ Y. Hasegawa, ${ }^{(28)}$

K. Hasuko, ${ }^{(28)}$ S. J. Hedges, ${ }^{(3)}$ S.S. Hertzbach, ${ }^{(16)}$ M.D. Hildreth, ${ }^{(26)}$ J. Huber, ${ }^{(19)}$

M.E. Huffer, ${ }^{(26)}$ E.W. Hughes, ${ }^{(26)}$ H. Hwang, ${ }^{(19)}$ Y. Iwasaki, ${ }^{(28)}$ D.J. Jackson, ${ }^{(24)}$

P. Jacques, ${ }^{(23)}$ J. A. Jaros, ${ }^{(26)}$ A.S. Johnson, ${ }^{(3)}$ J.R. Johnson, ${ }^{(31)}$ R.A. Johnson, ${ }^{(7)}$

T. Junk, ${ }^{(26)}$ R. Kajikawa, ${ }^{(18)}$ M. Kalelkar, ${ }^{(23)}$ H. J. Kang, ${ }^{(25)}$ I. Karliner, ${ }^{(13)}$

H. Kawahara, ${ }^{(26)}$ H.W. Kendall, ${ }^{(15)}$ Y. D. Kim, ${ }^{(25)}$ M.E. King, ${ }^{(26)}$ R. King, ${ }^{(26)}$

R.R. Kofler, ${ }^{(16)}$ N.M. Krishna, ${ }^{(9)}$ R.S. Kroeger, ${ }^{(17)}$ J.F. Labs, ${ }^{(26)}$ M. Langston, ${ }^{(19)}$

A. Lath, ${ }^{(15)}$ J.A. Lauber, ${ }^{(9)}$ D.W.G.S. Leith, ${ }^{(26)}$ V. Lia, ${ }^{(15)}$ M.X. Liu, ${ }^{(32)}$ X. Liu, ${ }^{(6)}$

M. Loreti, ${ }^{(20)}$ A. Lu, ${ }^{(5)}$ H.L. Lynch, ${ }^{(26)}$ J. Ma, ${ }^{(30)}$ G. Mancinelli, ${ }^{(21)}$ S. Manly, ${ }^{(32)}$

G. Mantovani, ${ }^{(21)}$ T.W. Markiewicz, ${ }^{(26)}$ T. Maruyama, ${ }^{(26)}$ H. Masuda, ${ }^{(26)}$

E. Mazzucato, ${ }^{(11)}$ A.K. McKemey, ${ }^{(4)}$ B.T. Meadows, ${ }^{(7)}$ R. Messner, ${ }^{(26)}$ P.M. Mockett, ${ }^{(30)}$

K.C. Moffeit, ${ }^{(26)}$ T.B. Moore, ${ }^{(32)}$ D. Muller, ${ }^{(26)}$ T. Nagamine, ${ }^{(26)}$ S. Narita, ${ }^{(28)}$

U. Nauenberg, ${ }^{(9)}$ H. Neal, ${ }^{(26)}$ M. Nussbaum, ${ }^{(7)}$ Y. Ohnishi, ${ }^{(18)}$ L.S. Osborne, ${ }^{(15)}$

R.S. Panvini, ${ }^{(29)}$ H. Park, ${ }^{(19)}$ T.J. Pavel, ${ }^{(26)}$ I. Peruzzi, ${ }^{(12)}{ }^{(b)}$ M. Piccolo, ${ }^{(12)}$

L. Piemontese, ${ }^{(11)}$ E. Pieroni, ${ }^{(22)}$ K.T. Pitts, ${ }^{(19)}$ R.J. Plano, ${ }^{(23)}$ R. Prepost, ${ }^{(31)}$

C.Y. Prescott, ${ }^{(26)}$ G.D. Punkar, ${ }^{(26)}$ J. Quigley, ${ }^{(15)}$ B.N. Ratcliff, ${ }^{(26)}$ T.W. Reeves, ${ }^{(29)}$ 
J. Reidy, ${ }^{(17)}$ P.E. Rensing, ${ }^{(26)}$ L.S. Rochester, ${ }^{(26)}$ P.C. Rowson, ${ }^{(10)}$ J.J. Russell, ${ }^{(26)}$

O.H. Saxton, ${ }^{(26)}$ T. Schalk, ${ }^{(6)}$ R.H. Schindler, ${ }^{(26)}$ B.A. Schumm, ${ }^{(14)}$ S. Sen, ${ }^{(32)}$

V.V. Serbo, ${ }^{(31)}$ M.H. Shaevitz, ${ }^{(10)}$ J.T. Shank, ${ }^{(3)}$ G. Shapiro, ${ }^{(14)}$ D.J. Sherden, ${ }^{(26)}$

K.D. Shmakov, ${ }^{(27)}$ C. Simopoulos, ${ }^{(26)}$ N.B. Sinev, ${ }^{(19)}$ S.R. Smith, ${ }^{(26)}$ M.B. Smy, ${ }^{(8)}$

J.A. Snyder, ${ }^{(32)}$ P. Stamer, ${ }^{(23)}$ H. Steiner, ${ }^{(14)}$ R. Steiner, ${ }^{(1)}$ M.G. Strauss, ${ }^{(16)}$ D. Su, ${ }^{(26)}$

F. Suekane, ${ }^{(28)}$ A. Sugiyama, ${ }^{(18)}$ S. Suzuki, ${ }^{(18)}$ M. Swartz, ${ }^{(26)}$ A. Szumilo, ${ }^{(30)}$

T. Takahashi, ${ }^{(26)}$ F.E. Taylor, ${ }^{(15)}$ E. Torrence, ${ }^{(15)}$ A.I. Trandafir, ${ }^{(16)}$ J.D. Turk, ${ }^{(32)}$

T. Usher, ${ }^{(26)} \mathrm{J}$. Va'vra, ${ }^{(26)} \mathrm{C}$. Vannini, ${ }^{(22)}$ E. Vella, ${ }^{(26)} \mathrm{J} . P$. Venuti, ${ }^{(29)} \mathrm{R}$. Verdier, ${ }^{(15)}$

P.G. Verdini, ${ }^{(22)}$ S.R. Wagner, ${ }^{(26)}$ A.P. Waite, ${ }^{(26)}$ S.J. Watts, ${ }^{(4)}$ A.W. Weidemann, ${ }^{(27)}$

E.R. Weiss, ${ }^{(30)}$ J.S. Whitaker, ${ }^{(\dot{3})}$ S.L. White, ${ }^{(27)}$ F.J. Wickens, ${ }^{(24)}$ D.A. Williams, ${ }^{(6)}$

D.C. Williams, ${ }^{(15)}$ S.H. Williams, ${ }^{(26)}$ S. Willocq, ${ }^{(32)}$ R.J. Wilson, ${ }^{(8)}$ W.J. Wisniewski, ${ }^{(26)}$

M. Woods, ${ }^{(26)}$ G.B. Word, ${ }^{(23)}$ J. Wyss, ${ }^{(20)}$ R.K. Yamamoto, ${ }^{(15)}$ J.M. Yamartino, ${ }^{(15)}$

X. Yang, ${ }^{(19)}$ S.J. Yellin, ${ }^{(5)}$ C.C. Young, ${ }^{(26)}$ H. Yuta, ${ }^{(28)}$ G. Zapalac, ${ }^{(31)}$ R.W. Zdarko, ${ }^{(26)}$

C. Zeitlin, ${ }^{(19)}$ and J. Zhou. ${ }^{(19)}$

(1) Adelphi University, Garden City, New York 11530.

(2) INFN Sezione di Bologna, I-40126 Bologna, Italy.

(3) Boston University, Boston, Massachusetts 02215.

${ }^{(4)}$ Brunel University, Uxbridge, Middlesex UB8 3PH, United Kingdom.

(5) University of California at Santa Barbara, Santa Barbara, California 93106.

(6) University of California at Santa Cruz, Santa Cruz, California 95064.

(7) University of Cincinnati, Cincinnati, Ohio 45221.

${ }^{(8)}$ Colorado State University, Fort Collins, Colorado 80523.

(9) University of Colorado, Boulder, Colorado 80309.

${ }^{(10)}$ Columbia University, New York, New York 10027.

(11) INFN Sezione di Ferrara and Università di Ferrara, I-44100 Ferrara, Italy.

(12) INFN Lab. Nazionali di Frascati, I-00044 Frascati, Italy.

(13) University of Illinois, Urbana, Illinois 61801.

(14) Lawrence Berkeley Laboratory, University of California, Berkeley, California 94720.

${ }^{(15)}$ Massachusetts Institute of Technology, Cambridge, Massachusetts 02139.

(16) University of Massachusetts, Amherst, Massachusetts 01003.

(17) University of Mississippi, University, Mississippi 38677.

${ }^{(18)}$ Nagoya University, Chikusa-ku, Nagoya 464 Japan.

(19) University of Oregon, Eugene, Oregon 97403.

(20) INFN Sezione di Padova and Università di Padova, I-35100 Padova, Italy. 
(21) INFN Sezione di Perugia and Università di Perugia, I-06100 Perugia, Italy.

(22) INFN Sezione di Pisa and Università di Pisa, I-56100 Pisa, Italy.

${ }^{(23)}$ Rutgers University, Piscataway, New Jersey 08855.

${ }^{(24)}$ Rutherford Appleton Laboratory, Chilton, Didcot, Oxon OX11 OQX United Kingdom.

(25) Sogang University, Seoul, Korea.

(26) Stanford Linear Accelerator Center, Stanford University, Stanford, California 94309.

(27) University of Tennessee, Knoxville, Tennessee 37996.

(28) Tohoku University, Sendai 980 Japan.

(29) Vanderbilt University, Nashville, Tennessee 37235.

(30) University of Washington, Seattle, Washington 98195.

(31) University of Wisconsin, Madison, Wisconsin 53706.

(32) Yale University, New Haven, Connecticut 06511.

${ }^{\dagger}$ Deceased.

(a) Also at the Università di Genova.

(b) Also at the Università di Perugia. 


\section{Appendix B}

\section{The Standard Model}

This appendix gives a brief overview of the Standard Model and a more detailed description of the Electroweak portions thereof.[3]

\section{B.1 An Overview of the Standard Model}

Our current understanding of the interactions of elementary particles and fields is usually described as the Standard Model of Particles and Fields. This model combines the unified Electroweak model of Glashow, Weinberg and Salam, describing weak and electromagnetic interactions, with Quantum Chromodynamics (QCD), describing the strong interactions. As such, it describes all the particles and interactions that we know of outside of gravity.

\section{B.1.1 Known Particles and Fields}

The Standard Model describes interactions between fermions mediated by gauge bosons. Fermions are particles of half integral spin, and bosons are particles with integral spin. We further divide the fermions into quarks (see Table B.1) which interact strongly and leptons (see Table B.2) which do not have strong interactions. The gauge bosons (see Table B.3) couple with the various fermions in different ways, for example, the photon couples to the electric charge. 
Table B.1: Known Quarks.

\begin{tabular}{l|c|c|l}
\hline Name & spin & charge & \multicolumn{1}{|c}{ mass[7] } \\
\hline $\mathrm{d}$ & $\frac{1}{2}$ & $-\frac{1}{3}$ & $5-15 \mathrm{MeV}$ \\
\hline $\mathrm{u}$ & $\frac{1}{2}$ & $\frac{2}{3}$ & $2-8 \mathrm{MeV}$ \\
\hline $\mathrm{s}$ & $\frac{1}{2}$ & $-\frac{1}{3}$ & $0.1-0.3 \mathrm{GeV}$ \\
\hline $\mathrm{c}$ & $\frac{1}{2}$ & $\frac{2}{3}$ & $1.0-1.6 \mathrm{GeV}$ \\
\hline $\mathrm{b}$ & $\frac{1}{2}$ & $-\frac{1}{3}$ & $4.1-4.5 \mathrm{GeV}$ \\
\hline $\mathrm{t}$ & $\frac{1}{2}$ & $\frac{2}{3}$ & $180 \pm 12 \mathrm{GeV}$ \\
\hline
\end{tabular}

Table B.2: Known Leptons.

\begin{tabular}{l|c|c|l}
\hline Name & spin & charge & \multicolumn{1}{|c}{ mass[7] } \\
\hline $\mathrm{e}$ & $\frac{1}{2}$ & -1 & $510.9991 \mathrm{keV}$ \\
\hline $\mathrm{v}_{\mathrm{e}}$ & $\frac{1}{2}$ & 0 & $<15 \mathrm{eV}$ \\
\hline$\mu$ & $\frac{1}{2}$ & -1 & $105.65839 \mathrm{MeV}$ \\
\hline$v_{\mu}$ & $\frac{1}{2}$ & 0 & $<0.17 \mathrm{MeV}$ \\
\hline$\tau$ & $\frac{1}{2}$ & -1 & $1777.0 \mathrm{MeV}$ \\
\hline$v_{\tau}$ & $\frac{1}{2}$ & 0 & $<24 \mathrm{MeV}$ \\
\hline
\end{tabular}

Table B.3: Known Gauge Bosons.

\begin{tabular}{l|c|c|l|l}
\hline \multicolumn{1}{c|}{ Name } & spin & charge & \multicolumn{1}{c}{ mass[7] } & \multicolumn{1}{c}{ force } \\
\hline$\gamma$ (photon) & 1 & 0 & $<6 \times 10^{-16} \mathrm{eV}$ & Electromagnetic \\
\hline $\mathrm{W}^{ \pm}$ & 1 & \pm 1 & $80.33 \pm 0.15 \mathrm{GeV}$ & Weak \\
\hline $\mathrm{Z}^{0}$ & 1 & 0 & $91.187 \pm 0.007 \mathrm{GeV}$ & Weak \\
\hline $\mathrm{g}$ (gluons) & 1 & 0 & 0 & Strong \\
\hline
\end{tabular}

Our observations also indicate that we can further categorize the known quarks and leptons into three generations (see Table B.4) which are identical in all respects other than 
mass. For example, we expect the electron, muon and tau to interact identically other than effects due to their differing masses. This expectation is commonly referred to as lepton universality, and many experiments have been conducted to test the truth of this hypothesis.

Table B.4: Generations of Fermions.

\begin{tabular}{c|c|c}
\hline 1st & 2nd & 3rd \\
\hline $\mathrm{e}$ & $\mu$ & $\tau$ \\
\hline$v_{\mathrm{e}}$ & $v_{\mu}$ & $v_{\tau}$ \\
\hline $\mathrm{u}$ & $\mathrm{c}$ & $\mathrm{t}$ \\
\hline $\mathrm{d}$ & $\mathrm{s}$ & $\mathrm{b}$ \\
\hline
\end{tabular}

\section{B.1.2 Interactions and Gauge Symmetries}

In quantum field theory, interactions derive from local gauge symmetries. Quantum Electrodynamics (QED) arises from a U(1) gauge symmetry and its associated coupling $\alpha(\mathrm{QED})$. The gauge symmetry gives rise to a conserved quantity, the electric charge, to which the associated gauge boson, the photon, couples. Likewise, QCD arises from an SU(3) gauge symmetry and its associated coupling $\alpha_{\mathrm{s}}$. The SU(3) symmetry of QCD also gives rise to several conserved quantities, the color charges, red, green and blue, to which the eight gluons couple. The electroweak interactions arise from an $S U(2) \otimes U(1)$ gauge symmetry and its associated couplings $g$ and $g^{\prime}$. This gauge symmetry includes the $U(1)$ gauge symmetry of QED as a subgroup, and the QED coupling can be derived from the electroweak couplings. Due to the process of spontaneous symmetry breaking, the electric charge is the only conserved quantity due to the electroweak gauge symmetry. This symmetry breaking also gives rise to the $\mathrm{W}$ and $\mathrm{Z}^{0}$ masses as well as the mass terms for all of the fermions in the theory. This will all be described in more detail in the next section. 


\section{B.2 Electroweak Theory}

The electroweak Standard Model describes the charged and neutral current weak interactions as well as the electromagnetic interactions. The weak fields are weak due to the large masses of their associated gauge bosons rather than to any inherent weakness of their couplings. These interactions derive from a $S U(2) \otimes U(1)$ gauge symmetry. The $S U(2)$ symmetry gives rise to the weak isospin $\mathbf{T}$ and is associated with the $\mathbf{W}^{\mu}$ fields and their the coupling $\mathrm{g}$. The $\mathrm{U}(1)$ symmetry gives rise to the weak hypercharge $\mathrm{Y}$ and is associated with the $\mathrm{B}^{\mu}$ field and its coupling $\mathrm{g}^{\prime}$. In this scheme, the fermions are arranged in weak isodoublets and singlets. Using the first generation as an example.

$$
\begin{aligned}
& \chi_{\mathrm{L}}=\left(\begin{array}{c}
\mathrm{v}_{\mathrm{e}} \\
\mathrm{e}
\end{array}\right)_{\mathrm{L}} \text { with } \mathrm{T}=\frac{1}{2} \text { and } \mathrm{Y}=-1 \\
& \Psi_{\mathrm{R}}=\mathrm{e}_{\mathrm{R}} \text { with } \mathrm{T}=0 \text { and } \mathrm{Y}=-2
\end{aligned}
$$

for the leptons and for the quarks,

$$
\begin{aligned}
& \chi_{\mathrm{L}}=\left(\begin{array}{l}
\mathrm{u} \\
\mathrm{d}
\end{array}\right)_{\mathrm{L}} \quad \text { with } \mathrm{T}=\frac{1}{2} \text { and } \mathrm{Y}=\frac{1}{3} \\
& \psi_{\mathrm{R}}=\mathrm{u}_{\mathrm{R}} \quad \text { with } \mathrm{T}=0 \text { and } \mathrm{Y}=\frac{4}{3} \text { or } \\
& \psi_{\mathrm{R}}=\mathrm{d}_{\mathrm{R}} \text { with } \mathrm{T}=0 \text { and } \mathrm{Y}=-\frac{2}{3}
\end{aligned}
$$

There is no $\psi_{\mathrm{R}}$ corresponding to the neutrino due to its masslessness. The weak isospin doublets correspond to left-handed helicity states, and the weak isosinglets correspond to right-handed helicity states. The left-handed component of a fermion may be written as $f_{\mathrm{L}}=\frac{1}{2}\left(1-\gamma^{5}\right) f$. Likewise, the right-handed component may be written $f_{\mathrm{R}}=\frac{1}{2}\left(1+\gamma^{5}\right) f$. We choose to define the weak isospin in this way because the experiments indicate that the charged current weak interaction couples only to left-handed fermions. We further define 
the weak hypercharge so that we can identify the combination $Q=T^{3}+\frac{Y}{2}$ with the electric charge.

\section{B.2.3 Massless Lagrangian}

$$
\begin{aligned}
\mathcal{L}= & \bar{\chi}_{\mathrm{L}} \gamma^{\mu}\left[\mathrm{i} \partial_{\mu}-\mathrm{g} \frac{1}{2} \tau \cdot \mathrm{W}_{\mu}-\mathrm{g}^{\prime}\left(-\frac{1}{2}\right) \mathrm{B}_{\mu}\right] \chi_{\mathrm{L}}+ \\
& \overline{\mathrm{e}}_{\mathrm{R}} \gamma^{\mu}\left[\mathrm{i} \partial_{\mu}-\mathrm{g}^{\prime}(-1) \mathrm{B}_{\mu}\right] \mathrm{e}_{\mathrm{R}}-\frac{1}{4} \mathbf{W}_{\mu \nu} \cdot \mathbf{W}^{\mu \nu}-\frac{1}{4} \mathrm{~B}_{\mu \nu} \cdot \mathrm{B}^{\mu \nu}
\end{aligned}
$$

We can write a Lagrangian (B.3) for the electroweak interactions of the lepton pair consisting of the electron and electron neutrino, where $\tau$ are the weak isospin matrices. This Lagrangian is gauge invariant, but contains no mass terms for the fermions or bosons as we would expect in reality. To add in the masses, we must add more terms, unfortunately, typical mass terms are not gauge invariant, so we must resort to using the Higgs mechanism to give masses to the particles in the theory.

\section{B.2.4 The Higgs Mechanism}

To give the gauge bosons mass, we add another term to the Lagrangian introducing four scalar fields

$$
\mathcal{L}^{\prime}=\left|\left(i \partial_{\mu}-\mathrm{g} \mathbf{T} \cdot \mathrm{W}_{\mu}-\mathrm{g}^{\prime} \frac{\mathrm{Y}}{2} \mathrm{~B}_{\mu}\right) \phi\right|^{2}-\mathrm{V}(\phi)
$$

This is gauge invariant if we choose the $\phi$ such that they belong to $S U(2) \otimes U(1)$ multiplets. We can choose four fields in an isospin doublet with weak hypercharge $\mathrm{Y}=1$.

$$
\begin{gathered}
\phi=\left(\begin{array}{c}
\phi^{+} \\
0
\end{array}\right) \text { with } \\
\phi^{+} \equiv\left(\dot{\phi}_{1}+i \phi_{2}\right) / \sqrt{2} \\
\phi^{0} \equiv\left(\phi_{3}+i \phi_{4}\right) / \sqrt{2}
\end{gathered}
$$


To generate masses, we choose $V(\phi)$ to be the Higgs potential,

$$
\mathrm{V}(\phi)=\mu^{2} \phi^{\dagger} \phi+\lambda\left(\phi^{\dagger} \phi\right)^{2}
$$

with $\mu^{2}<0$ and $\lambda>0$ and choose a minimum of $V(\phi)$ as the new vacuum expectation value to expand the Lagrangian about. We choose the minimum at

$$
\phi_{0} \equiv \sqrt{\frac{1}{2}}\left(\begin{array}{l}
0 \\
v
\end{array}\right)
$$

This breaks the gauge symmetry and allows us to have massive gauge bosons. It also has the property of leaving a $U(1)$ subgroup of the $S U(2) \otimes U(1)$ symmetry unbroken. This subgroup is generated by the combination of operators

$$
\mathrm{Q} \equiv \mathrm{T}^{3}+\frac{\mathrm{Y}}{2}
$$

which we associate with the electric charge, which remains as the only unbroken symmetry in the theory. Expanding the mass term gives

$$
\begin{aligned}
& \mid\left(-\mathrm{ig} \frac{\tau}{2} \cdot \mathrm{W}_{\mu}-\mathrm{i} \frac{\mathrm{g}^{\prime}}{2} \mathrm{~B}_{\mu}\right) \phi^{2}= \\
& \quad\left(\frac{1}{2} \mathrm{vg}\right)^{2} \mathrm{~W}_{\mu}^{+} \mathrm{W}^{-\mu}+\frac{1}{8} \mathrm{v}^{2}\left(\mathrm{~W}_{\mu}^{3}, \mathrm{~B}_{\mu}\right)\left[\begin{array}{cc}
\mathrm{g}^{2} & -\mathrm{gg} \mathrm{g}^{\prime} \\
-\mathrm{gg}^{\prime} & \mathrm{g}^{\prime 2}
\end{array}\right]\left(\begin{array}{c}
\mathrm{W}^{3 \mu} \\
\mathrm{B}^{\mu}
\end{array}\right)
\end{aligned}
$$

Where we have defined

$$
\mathrm{W}_{\mu}^{ \pm}=\frac{\mathrm{W}_{\mu}^{1} \mp i \mathrm{~W}_{\mu}^{2}}{\sqrt{2}}
$$

This gives $\frac{1}{2} \mathrm{vg}$ as the $\mathrm{W}$ mass since we expect the mass of a charged boson to derive from a term of the form $\mathrm{M}^{2} \mathrm{~W}^{+} \mathrm{W}$. Likewise, we expect neutral bosons to have mass terms of the form $\frac{1}{2} M^{2} Z^{2}$. The second term in (B.9) can be written as 


$$
\begin{aligned}
\frac{1}{8} v^{2}\left[g^{2}\left(W^{3}\right)^{2}-2 g g^{\prime} W_{\mu}^{3} B^{\mu}+g^{\prime 2} B^{2}\right]= \\
\frac{1}{8} v^{2}\left[g W_{\mu}^{3}-g^{\prime} B_{\mu}\right]^{2}+0\left[g^{\prime} W_{\mu}^{3}+g B_{\mu}\right]^{2}
\end{aligned}
$$

The first term is what we identify with the $\mathrm{Z}^{0}$ boson and we add in the second term (multiplied by zero) for the combination of the $\mathrm{W}^{3}$ and $\mathrm{B}$ fields orthogonal to the $\mathrm{Z}^{0}$, which we associate with the massless photon. This illustrates that in this formalism, we have given mass to the charged and neutral weak bosons, while leaving the photon massless. We may add further gauge invariant terms in the $\phi$ field to give masses to the fermions. Unfortunately, these terms are arbitrary and do not give any insight into the actual masses of the fermions. The addition of the $\phi$ field also gives rise to at least one new Boson in the electroweak model, the Higgs boson. The formalism above is the minimal version required to give masses to the weak gauge bosons and fermions and gives rise to a single neutral Higgs boson. More complicated formulations $\phi$ field can accomplish the addition of masses accompanied by greater numbers of Higgs particles. At least one Higgs boson is required in the Standard Model. At this time, the Higgs remains undetected, but other electroweak measurements give limits for its allowed mass.

\section{B.2.5 Electroweak Mixing}

Above, we see that when we use the Higgs mechanism to add mass to the weak gauge bosons, we get mixing of the two neutral electroweak fields. We refer to this as electroweak mixing. This is the heart of the Weinberg-Salam model of the electroweak interactions. When we properly normalize the weak fields, we get 


$$
\begin{aligned}
& A_{\mu}=\frac{g^{\prime} W_{\mu}^{3}+g B_{\mu}}{\sqrt{g^{2}+g^{\prime 2}}} \text { with } M_{A}=0, \text {, and } \\
& Z_{\mu}=\frac{g_{\mu}^{3}-g^{\prime} B_{\mu}}{\sqrt{g^{2}+g^{\prime 2}}} \text { with } M_{Z}=\frac{1}{2} v \sqrt{g^{2}+g^{\prime 2}}
\end{aligned}
$$

We can rewrite this in the more traditional mixing notation by defining the weak mixing angle commonly referred to as the Weinberg angle

$$
\tan \theta_{\mathrm{W}}=\frac{\mathrm{g}^{\prime}}{\mathrm{g}}
$$

This gives

$$
\begin{aligned}
& \mathrm{A}_{\mu}=\cos \theta_{\mathrm{W}} \mathrm{B}_{\mu}+\sin \theta_{\mathrm{W}} \mathrm{W}_{\mu}^{3} \\
& \mathrm{Z}_{\mu}=-\sin \theta_{\mathrm{W}} \mathrm{B}_{\mu}+\cos \theta_{\mathrm{W}} \mathrm{W}_{\mu}^{3}
\end{aligned}
$$

\subsubsection{Feynman Rules for the Electroweak Interactions}

From the Electroweak Lagrangian, we can work out the Feynman rules for the various electroweak interactions.
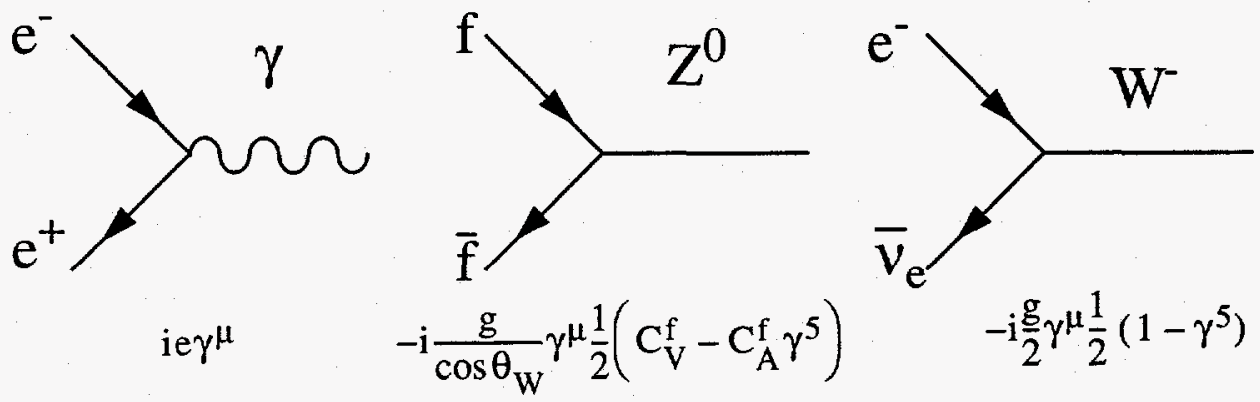

Figure B.1: Electroweak vertices and couplings. 
For the electromagnetic interaction, we get the familiar coupling of ie $\gamma^{\mu}$, where $e$ is the electron charge, and is equal to $\mathrm{g} \cdot \sin \theta_{\mathrm{W}}$. For the charged current weak interaction with leptons, we get a coupling of $-\mathrm{i} \frac{\mathrm{g}}{2} \gamma^{\mu} \frac{1}{2}\left(1-\gamma^{5}\right)$. This is generally referred to as a V-A interaction as the $\gamma^{\mu}$ picks out the vector current coupling of the two fermions, and the $\gamma^{\mu} \gamma^{5}$ picks out the axial vector current coupling of the two fermions to the $\mathrm{W}$. This is the source of parity violation in charged weak interactions as the vector and axial vector currents have opposite behavior under parity inversion. For the neutral current weak interactions, we get a coupling of $-i \frac{g}{\cos \theta_{W}} \gamma^{\mu} \frac{1}{2}\left(C_{V}^{f}-C_{A}^{f} \gamma^{5}\right)$. Where we define

$$
\begin{aligned}
& C_{V}^{f}=T_{f}^{3}-2 \sin ^{2} \theta_{W} Q_{f} \\
& C_{A}^{f}=T_{f}^{3}
\end{aligned}
$$

Where $\mathrm{T}$ is the weak isospin and $\mathrm{Q}$ is the charge of the interacting fermion.

Table B.5: Fermion Properties Relevant to the $\mathbf{Z}^{\mathbf{0}}$.

\begin{tabular}{l|c|c|c}
\hline \multicolumn{1}{c|}{ fermion } & $\mathrm{Q}_{\mathrm{f}}$ & $\left(\mathrm{T}_{\mathrm{f}}^{3}\right)_{\mathrm{L}}$ & $\left(\mathrm{T}_{\mathrm{f}}^{3}\right)_{\mathrm{R}}$ \\
\hline $\mathrm{u}, \mathrm{c}, \mathrm{t}$ & $+\frac{2}{3}$ & $+\frac{1}{2}$ & 0 \\
\hline $\mathrm{d}, \mathrm{s}, \mathrm{b}$ & $-\frac{1}{3}$ & $-\frac{1}{2}$ & 0 \\
\hline$v_{\mathrm{e}}, v_{\mu}, v_{\tau}$ & 0 & $+\frac{1}{2}$ & N/A \\
\hline $\mathrm{e}, \mu, \tau$ & -1 & $-\frac{1}{2}$ & 0 \\
\hline
\end{tabular}




\section{Appendix C}

\section{Tau Decay Parameterization for a Generalized Weak Interaction}

We can write a generalized weak decay matrix element for leptonic tau decays as follows

$$
\begin{aligned}
& \frac{{ }^{4 G_{F}}}{\sqrt{2}} \sum_{\gamma=S, V, T} g_{\varepsilon \mu}^{\gamma}\left\langle\bar{\ell}_{\varepsilon}\left|\Gamma^{\gamma}\right|\left(\nu_{\ell}\right)_{\mathrm{n}}\right\rangle\left\langle\left(\bar{v}_{\tau}\right)_{\mathrm{m}}\left|\Gamma_{\gamma}\right| \tau_{\mu}\right\rangle \\
& \varepsilon, \mu=\mathrm{R}, \mathrm{L}
\end{aligned}
$$

using the notation of Fetscher, et al.[39][6] $\gamma=\mathrm{S}, \mathrm{V}$, or T represent the type of the interaction, scalar, vector or tensor, and $\varepsilon, \mu=\mathrm{R}$ or $\mathrm{L}$ indicate the chiralities of the tau and resulting decay lepton. The chiralities of the neutrinos, $m$ and $n$ are determined by $\gamma, \varepsilon$, and $\mu$. In this parameterization, $\mathrm{G}_{\mathrm{F}}$ and the complex amplitudes $\mathrm{g}_{\varepsilon \mu}^{\gamma}$ determine the interaction. In the Standard Model, we have a pure V-A coupling which gives us $\mathrm{g}_{\mathrm{LL}}^{\mathrm{V}}=1$, and the remaining amplitudes all identically zero. We can relate these couplings to the general parameterization from Section 1.3 by

$$
\begin{gathered}
\rho-\frac{3}{4}=\frac{3}{4}\left(\frac{-a+2 c}{A}\right), \\
\eta=\frac{\alpha-2 \beta}{A} \\
\eta^{\prime \prime}=\frac{3 \alpha+2 \beta}{A} \\
\delta-\frac{3}{4}=\frac{9}{4}\left(\frac{a^{\prime}-2 c^{\prime}}{A-a-3 a^{\prime}-4\left(b+b^{\prime}\right)-6 c+14 c^{\prime}}\right),
\end{gathered}
$$




$$
\begin{gathered}
1-\xi \frac{\delta}{\rho}=4 \frac{\left(b+b^{\prime}\right)+2\left(c-c^{\prime}\right)}{A-a+2 c}, \\
1-\xi^{\prime}=\frac{\left(a+a^{\prime}\right)+4\left(b+b^{\prime}\right)+6\left(c+c^{\prime}\right)}{A}, \text { and } \\
1-\xi^{\prime \prime}=\frac{-2 a+20 c}{A}, \text { where } \\
A=a+4 b+6 c
\end{gathered}
$$

Where these are related to the complex couplings by

$$
\begin{aligned}
& a=16\left(\left|g_{R L}^{V}\right|^{2}+\left|g_{L R}^{V}\right|^{2}\right)+\left|g_{R L}^{S}+6 g_{R L}^{T}\right|^{2}+\left|g_{L R}^{S}+6 g_{L R}^{T}\right|^{2}, \\
& a^{\prime}=16\left(\left|g_{R L}^{V}\right|^{2}-\left|g_{L R}^{V}\right|^{2}\right)+\left|g_{R L}^{S}+6 g_{R L}^{T}\right|^{2}-\left|g_{L R}^{S}+6 g_{L R}^{T}\right|^{2}, \\
& \alpha=8 \operatorname{Re}\left[\mathrm{g}_{\mathrm{RL}}^{\mathrm{V}}\left(\mathrm{g}_{\mathrm{LR}}^{\mathrm{S}}+6 \mathrm{~g}_{\mathrm{LR}}^{\mathrm{T}}\right) * \mathrm{~g}_{\mathrm{LR}}^{\mathrm{V}}\left(\mathrm{g}_{\mathrm{RL}}^{\mathrm{S}}+6 \mathrm{~g}_{\mathrm{RL}}^{\mathrm{T}}\right) *\right] \text {, } \\
& \alpha^{\prime}=8 \operatorname{Im}\left[-g_{\mathrm{RL}}^{\mathrm{V}}\left(\mathrm{g}_{\mathrm{LR}}^{S}+6 \mathrm{~g}_{\mathrm{LR}}^{\mathrm{T}}\right)^{*}-\mathrm{g}_{\mathrm{LR}}^{\mathrm{V}}\left(\mathrm{g}_{\mathrm{RL}}^{\mathrm{S}}+6 \mathrm{~g}_{\mathrm{RL}}^{\mathrm{T}}\right)^{*}\right] \text {, } \\
& \mathrm{b}=4\left(\left|\mathrm{~g}_{\mathrm{RR}}^{\mathrm{V}}\right|^{2}+\left|\mathrm{g}_{\mathrm{LL}}^{\mathrm{V}}\right|^{2}\right)+\left|\mathrm{g}_{\mathrm{RR}}^{\mathrm{S}}\right|^{2}+\left|\mathrm{g}_{\mathrm{LL}}^{\mathrm{S}}\right|^{2}, \\
& b^{\prime}=4\left(\left|g_{R R}^{V}\right|^{2}-\left|g_{L L}^{V}\right|^{2}\right)+\left|g_{R R}^{S}\right|^{2}-\left|g_{L L}^{S}\right|^{2}, \\
& \beta=-4 \operatorname{Re}\left[\mathrm{g}_{\mathrm{RR}}^{\mathrm{V}}\left(\mathrm{g}_{\mathrm{LL}}^{S}\right)^{*}+\mathrm{g}_{\mathrm{LL}}^{\mathrm{V}}\left(\mathrm{g}_{\mathrm{RR}}^{\mathrm{S}}\right)^{*}\right] \\
& \beta^{\prime}=4 \operatorname{Im}\left[g_{\mathrm{RR}}^{\mathrm{V}}\left(\mathrm{g}_{\mathrm{LL}}^{S}\right)^{*}-\mathrm{g}_{\mathrm{LL}}^{\mathrm{V}}\left(\mathrm{g}_{\mathrm{RR}}^{S}\right)^{*}\right] \text {, } \\
& c=\frac{1}{2}\left[\left|g_{R L}^{S}-2 g_{R L}^{T}\right|^{2}+\left|g_{L R}^{S}-2 g_{L R}^{T}\right|^{2}\right] \text {, and } \\
& c^{\prime}=\frac{1}{2}\left[\left|g_{R L}^{S}-2 g_{R L}^{T}\right|^{2}-\left|g_{L R}^{S}-2 g_{L R}^{T}\right|^{2}\right]
\end{aligned}
$$


This gives some rather complex relations between the Michel parameters and the generalized coupling constants which are not particularly illuminating, but are included here for completeness' sake.[40] 


\section{Appendix D}

\section{Tau Neutrino Helicity from Leptonic Tau Decays}

There are other possible parameterizations for the energy spectrum of leptons from tau decays. One of the more interesting ones is using a single parameter representing the helicity of the tau neutrino.[41] In this parameterization, we assume that the W couplings to the electron and muon are the Standard Model V-A couplings and allow the W coupling of the tau to be a combination of $\mathrm{V}$ and $\mathrm{A}$. We can define a quantity

$$
h_{v}=\frac{2 C_{V}^{W} C_{A}^{W}}{\left(C_{V}^{W}\right)^{2}+\left(C_{A}^{W}\right)^{2}}
$$

This can be interpreted as twice the helicity of the tau neutrino, which we expect to be lefthanded (helicity $=-\frac{1}{2}$ ) in the Standard Model. If we take all other couplings of the W to the tau to be zero, the Michel Parameters become:

$$
\begin{gathered}
\rho=\frac{3}{8}\left(1-h_{v}\right) \\
\xi=h_{v}+2 \\
\xi \delta=\frac{3}{8}\left(1-h_{v}\right), \text { and } \\
\eta=0
\end{gathered}
$$

We can redo the fit as a one parameter fit, giving us values for the tau neutrino helicity 
from leptonic tau decays.

Table D.1: Leptonic $h_{v}$ Results.

\begin{tabular}{c|c|c}
\hline Combined & Electrons & Muons \\
\hline$-0.97 \pm 0.21$ & $-0.91 \pm 0.31$ & $-1.02 \pm 0.28$ \\
\hline
\end{tabular}

We can also evaluate the systematics for these measurements:

Table D.2: Leptonic $h_{v}$ Systematics.

\begin{tabular}{l|r|r|r}
\hline \multicolumn{1}{c|}{ Effect } & Combined & Electrons & \multicolumn{1}{c}{ Muons } \\
\hline $\mathrm{A}_{\ell}$ & 0.013 & 0.012 & 0.014 \\
\hline Radiation & 0.005 & 0.008 & 0.003 \\
\hline Beam Polarization & 0.003 & 0.004 & 0.003 \\
\hline Beam Energy & 0.027 & 0.028 & 0.025 \\
\hline Non- $\tau$ Background & 0.014 & 0.016 & 0.021 \\
\hline$\tau$ Background & 0.008 & 0.013 & 0.009 \\
\hline Event Selection & 0.022 & 0.049 & 0.005 \\
\hline Momentum Resolution & 0.041 & 0.079 & 0.037 \\
\hline Angular Resolution & 0.000 & 0.000 & 0.000 \\
\hline Total & 0.058 & 0.101 & 0.053 \\
\hline
\end{tabular}

This gives us a combined $h_{v}$ result of $-0.97 \pm 0.21 \pm 0.06$, which agrees quite well with the standard model and other measurements of this quantity including the SLD $h_{v}$ measurement utilizing taus decaying to pions and rho mesons of $h_{v}=-0.93 \pm 0.10 \pm 0.04 .[42]$ 


\section{Appendix E}

\section{LAC Electro-Magnetic Energy Calibration}

For the purposes of measuring electromagnetic shower energies, the LAC response is significantly affected by the presence of the support washer for the LAC cryostat and various material in the endcap regions. Thus, there is significant variation in the LAC's response to electromagnetic showers. Most of this material has the same cylindrical symmetry as the SLD itself, so we can correct for it as a function of polar angle. We calibrate this by selecting a sample of wide angle Bhabha (WAB) events. Since all of the energy in these events should be electromagnetic (electrons, positrons or photons) we expect that the total electromagnetic energy in each event to be the same as the sum of the beam energies. For non-radiative events, we can use the individual tracks, which should have energy equal to the beam energy, to calibrate the EM response of the LAC. We select events with exactly one track in each hemisphere with the tracks having opposite charges and event's total EM LAC energy $>50 \mathrm{GeV}$ (Min-I scale). This gives us a total sample of 5963 WAB events from the 1993-95 data. The LAC energy distribution for clusters associated with the tracks is shown in Fig. E.1. Here it is easy to see the effects of the LAC support washers at $\sim \pm 0.45$ in $\cos \theta$, the degradation of the LAC performance near the endcap region, the barrel-endcap overlap region, and the LAC's projective tower geometry. 


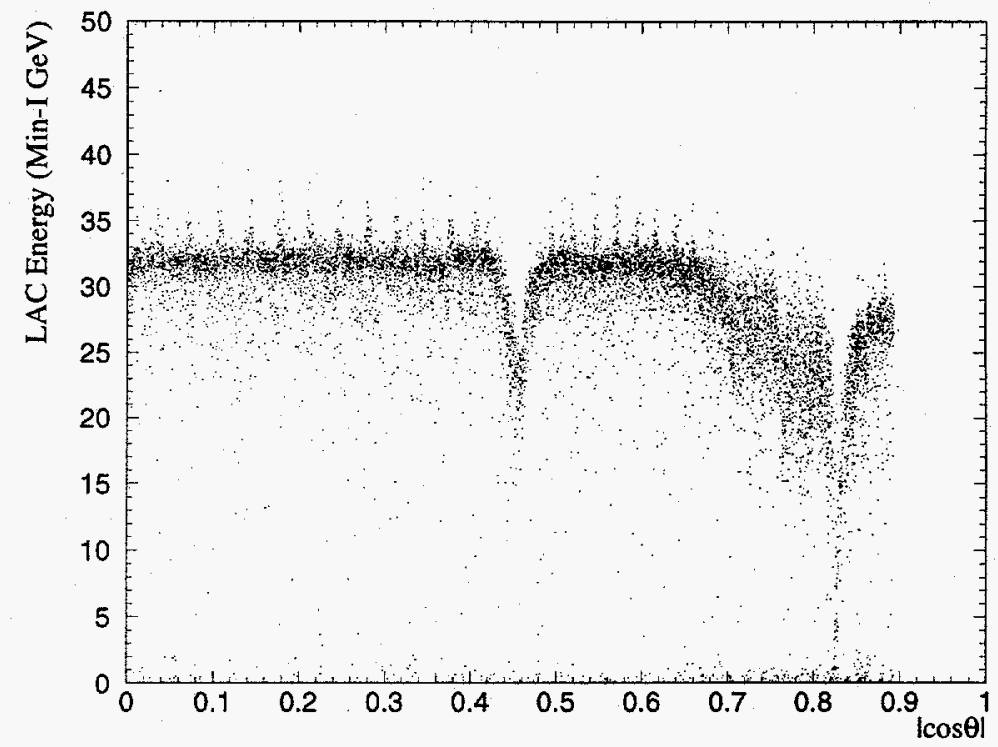

Figure E.1: EM LAC response for wide angle Bhabha events. (Min-I GeV)

Using this data, we generate a calibration of the LAC's EM scale as a function of polar angle. This permits us to do a better job of rejecting WAB events in the washer and endcap regions, where using the uncorrected LAC energy will lead to some leakage in these regions. A similar process is done using the Monte Carlo, yielding similar results. 


\section{References}

[1] L. Michel, Proc. Phys. Soc. A63, 514 (1950);

C. Bouchiat and L. Michel, Phys. Rev. 106, 170 (1957).

[2] M. Swartz, SLD Physics Note \#21, April 1993.

[3] F. Halzen and A. Martin, Quarks and Leptons: An Introductory Course in Modern Particle Physics, J. Wiley and Sons, 1984.

[4] W. Greiner and B Möller, Gauge Theory of Weak Interactions, Springer-Verlag, 1993.

[5] Y.S. Tsai, Phys. Rev. D4, 2821 (1971).

[6] W. Fetscher, and H.J. Gerber, Phys. Rev. D54, 251 (1996).

[7] Particle Data Group, R.M. Barnett et al., Phys. Rev. D54 (1996).

[8] ALEPH Collab., D. Buskulic et al., Phys. Lett. B346, 379 (1995).

[9] CLEO Collab., R. Ammar et al., CLNS-96-1429 (1996).

[10] L3 Collab., M. Acciarri et al., Phys. Lett. B377, 313 (1996).

[11] ARGUS Collab., H. Albrecht et al., Phys. Lett. B341, 441 (1995); B349, 576 (1995).

[12] N. Phinney, Int. J. Mod. Phys A, Proc. Supl. 2A, 45 (1993).

[13] R. Prepost, Polarized Electron Sources, Unpublished.

[14] G. Blaylock, SLD Physics Note \#22, 1993.

[15] E. Torrence, Ph.D. Thesis, Massachusetts Institute of Technology, 1997.

[16] SLD Design Report, SLAC-REPORT-273, May 1984.

[17] C.J.S. Damerell et. al., Proceedings of the 26th ICHEP, Dallas, Texas, August 1992; M.Strauss et. al., Proceedings of the 27th ICHEP, Glasgow, July 1994. 
[18] M. Fero et al., Nucl. Instr. Meth. A328, 111 (1995).

[19] K. Abe et al., Nucl. Instr. Meth. A343, 74 (1994).

[20] K.G. Baird, Ph.D. Thesis, Rutgers University, 1996.

[21] D. Axen et al., Nucl. Instr. Meth. A328, 472 (1993).

[22] A.C. Benvenuti et al., Nucl. Instr. Meth. A290, 353 (1990).

[23] D.C. Williams, Ph.D. Thesis, Massachusetts Institute of Technology, 1994.

[24] J.J. Russell, private communication.

[25] S. Jadach, B.F.L. Ward, and Z. Was, Comp. Phys. Comm. 79, 503 (1994).

[26] GEANT program, version 3.21, CERN Program Library (1994).

[27] A. Lath, Ph.D. Thesis, Massachusetts Institute of Technology, 1994.

[28] H. Anlauf et al., Comp.Phys. Comm. 79, 466 (1994).

[29] T. Sjöstrand, Comp. Phys. Comm. 82, 74 (1994).

[30] H. Aihara et al., Phys. Rev. Lett. 58, 97 (1987); and references therein.

[31] R. King, Ph.D. Thesis, Stanford University, 1994.

[32] Long Writeup D506, MINUIT Version 94.1, CERN Program Library (1994).

[33] W.H. Press, S.A. Teukolsky, W.T. Vetterling, and B.P. Flannery, Numerical Recipes in C: The Art of Scientific Computing, Cambridge University Press, 1992.

[34] Long Writeup Q121, CERN Program Library (1994).

[35] M. Peskin, private communication.

[36] SLD Collab., K. Abe et al., Phys. Rev. Lett. 78, 2075 (1997).

[37] Z. Was and B.F.L. Ward, private communications.

[38] SLD Collab., K. Abe et el., SLAC-PUB-7333, accepted by Phys. Rev. Lett. (1997).

[39] W. Fetscher, H.J. Gerber, and K.F. Johnson, Phys. Lett B173, 102 (1986).

[40] K. Mursula, M. Roos, and F. Scheck, Nucl. Phys. B219, 321 (1989). 
[41] P. Privitera, Phys. Lett. B288, 227 (1992).

[42] N.J. Allen, Ph.D. Thesis, Brunel University, 1997.

[43] N. Allen, M. Daoudi, E. Etzion, J. Johnson, A. McKemey and J. Quigley, SLD Physics Note \# 54, Septermber 1996. 